\title{
eCommons@AKU
}

Department of Paediatrics and Child Health

Division of Woman and Child Health

October 2008

\section{Fluoroquinolones for treating typhoid and paratyphoid fever (enteric fever)}

Durrane Thaver

Aga Khan University

Anita K. M. Zaidi

Aga Khan University, anita.zaidi@aku.edu

Julia A. Critchley

Asma Azmatullah

Aga Khan University

Syed Ali Madni

See next page for additional authors

Follow this and additional works at: https://ecommons.aku.edu/ pakistan_fhs_mc_women_childhealth_paediatr

Part of the Infectious Disease Commons, and the Pediatrics Commons

\section{Recommended Citation}

Thaver, D., Zaidi, A. K., Critchley, J., Azmatullah, A., Madni, S., Bhutta, Z. A. (2008). Fluoroquinolones for treating typhoid and paratyphoid fever (enteric fever). Cochrane Database of Systematic Reviews(4), 4530.

Available at: https://ecommons.aku.edu/pakistan_fhs_mc_women_childhealth_paediatr/79 
Authors

Durrane Thaver, Anita K. M. Zaidi, Julia A. Critchley, Asma Azmatullah, Syed Ali Madni, and Zulfiqar Ahmed Bhutta 


\section{Fluoroquinolones for treating typhoid and paratyphoid fever (enteric fever) (Review)}

Thaver D, Zaidi AKM, Critchley JA, Azmatullah A, Madni SA, Bhutta ZA

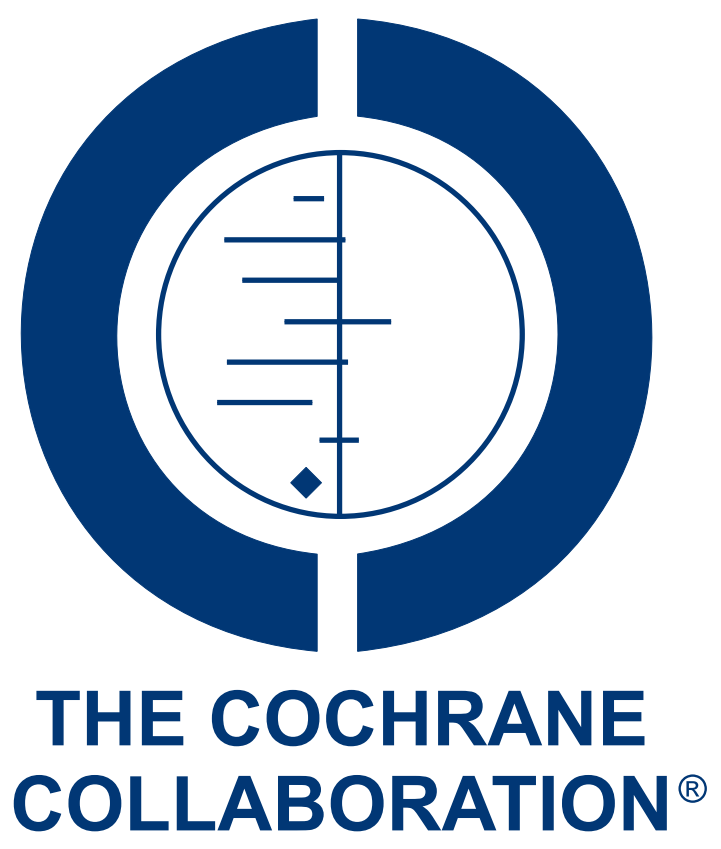

This is a reprint of a Cochrane review, prepared and maintained by The Cochrane Collaboration and published in The Cochrane Library 2008, Issue 4

http://www.thecochranelibrary.com

\section{WILEY}

Fluoroquinolones for treating typhoid and paratyphoid fever (enteric fever) (Review)

Copyright @ 2008 The Cochrane Collaboration. Published by John Wiley \& Sons, Ltd. 
TABLE OF CONTENTS

HEADER . . . . . . . . . . . . . . . . . . . . . . . . . . . . . . . . . . . . . . .

ABSTRACT . . . . . . . . . . . . . . . . . . . . . . . . . . . . . . . . . . . . . . . . . . 1

PLAIN LANGUAGE SUMMARY . . . . . . . . . . . . . . . . . . . . . . . . . . . . . . . . . . . 2

BACKGROUND . . . . . . . . . . . . . . . . . . . . . . . . . . . . . . . . . . . . . 2

OBJECTIVES . . . . . . . . . . . . . . . . . . . . . . . . . . . . . . . . . . . . . . . . . . . . . 4.4

METHODS . . . . . . . . . . . . . . . . . . . . . . . . . . . . . . . . . . . . . . 44

RESULTS . . . . . . . . . . . . . . . . . . . . . . . . . . . . . . . . . . . . . . . 413

Figure 1. . . . . . . . . . . . . . . . . . . . . . . . . . . . . . . . . . . . . . 16

Figure 2. . . . . . . . . . . . . . . . . . . . . . . . . . . . . . . . . . . . . . . 17

Figure 3. . . . . . . . . . . . . . . . . . . . . . . . . . . . . . . . . . . . . . 25

Figure 4. . . . . . . . . . . . . . . . . . . . . . . . . . . . . . . . . . . . . . . . . . . .

Figure 5. . . . . . . . . . . . . . . . . . . . . . . . . . . . . . . . . . . . . . $\quad 27$

Figure 6. . . . . . . . . . . . . . . . . . . . . . . . . . . . . . . . . . . . . . 29

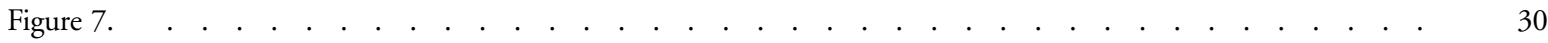

Figure 8. . . . . . . . . . . . . . . . . . . . . . . . . . . . . . . . . . . . . . .

DISCUSSION . . . . . . . . . . . . . . . . . . . . . . . . . . . . . . . . . . . . . 34

AUTHORS' CONCLUSIONS . . . . . . . . . . . . . . . . . . . . . . . . . . . . . . . . . . . . . .

ACKNOWLEDGEMENTS . . . . . . . . . . . . . . . . . . . . . . . . . . . . . . . . . . . . . .

REFERENCES . . . . . . . . . . . . . . . . . . . . . . . . . . . . . . . . . . . . . . 38

CHARACTERISTICS OF STUDIES . . . . . . . . . . . . . . . . . . . . . . . . . . . . . . . . . . . . . . . . 44

DATA AND ANALYSES . . . . . . . . . . . . . . . . . . . . . . . . . . . . . . . . . . . . . . . . . . . . 72

Analysis 1.1. Comparison 1 Fluoroquinolones vs chloramphenicol, Outcome 1 Clinical failure. . . . . . . . . . 80

Analysis 1.2. Comparison 1 Fluoroquinolones vs chloramphenicol, Outcome 2 Microbiological failure. . . . . . 82

Analysis 1.3. Comparison 1 Fluoroquinolones vs chloramphenicol, Outcome 3 Relapse. . . . . . . . . . . . $\quad$. 83

Analysis 1.4. Comparison 1 Fluoroquinolones vs chloramphenicol, Outcome 4 Fever clearance time. . . . . . . . . 84

Analysis 1.5. Comparison 1 Fluoroquinolones vs chloramphenicol, Outcome 5 Length of hospital stay. . . . . . 85

Analysis 1.6. Comparison 1 Fluoroquinolones vs chloramphenicol, Outcome 6 Convalescent faecal carriage $(\mathrm{NaR}$ not

reported and MDR absent). . . . . . . . . . . . . . . . . . . . . . . . . . . . . . 86

Analysis 1.7. Comparison 1 Fluoroquinolones vs chloramphenicol, Outcome 7 Complications. . . . . . . . . . 86

Analysis 1.8. Comparison 1 Fluoroquinolones vs chloramphenicol, Outcome 8 Adverse events (not serious). $\quad$. $\quad$. $\quad 87$

Analysis 2.1. Comparison 2 Fluoroquinolones vs amoxicillin (AMX) or ampicillin (AMP), Outcome 1 Clinical failure

(MDR and $\mathrm{NaR}$ not reported). . . . . . . . . . . . . . . . . . . . . . . . . . . . . . . . . . . . . . . . 88

Analysis 2.2. Comparison 2 Fluoroquinolones vs amoxicillin (AMX) or ampicillin (AMP), Outcome 2 Microbiological

failure (MDR and $\mathrm{NaR}$ not reported).

Analysis 2.3. Comparison 2 Fluoroquinolones vs amoxicillin (AMX) or ampicillin (AMP), Outcome 3 Adverse events (not

serious) (MDR and $\mathrm{NaR}$ not reported). . . . . . . . . . . . . . . . . . . . . . . . . . . . . . . . 89

Analysis 3.1. Comparison 3 Fluoroquinolones vs co-trimoxazole, Outcome 1 Clinical failure (MDR absent). $\quad . \quad$. $\quad 90$

Analysis 3.2. Comparison 3 Fluoroquinolones vs co-trimoxazole, Outcome 2 Microbiological failure (MDR absent). 91

Analysis 3.3. Comparison 3 Fluoroquinolones vs co-trimoxazole, Outcome 3 Adverse events (not serious) (MDR absent). 92

Analysis 4.1. Comparison 4 Fluoroquinolones vs azithromycin, Outcome 1 Clinical failure (in adults). . . . . . 93

Analysis 4.2. Comparison 4 Fluoroquinolones vs azithromycin, Outcome 2 Microbiological failure (in adults). $\quad .94$

Analysis 4.3. Comparison 4 Fluoroquinolones vs azithromycin, Outcome 3 Relapse (in adults). . . . . . . . . . . 95

Analysis 4.4. Comparison 4 Fluoroquinolones vs azithromycin, Outcome 4 Fever clearance time (in adults). $\quad$. $\quad$. 96

Analysis 4.5. Comparison 4 Fluoroquinolones vs azithromycin, Outcome 5 Length of hospital stay (days) (in adults). 97

Analysis 4.6. Comparison 4 Fluoroquinolones vs azithromycin, Outcome 6 Convalescent faecal carriage (in adults). . 98

Analysis 4.7. Comparison 4 Fluoroquinolones vs azithromycin, Outcome 7 Complications (in adults). . . . . . . . 99

Analysis 4.8. Comparison 4 Fluoroquinolones vs azithromycin, Outcome 8 Clinical failure (mostly children). . . . . 99

Analysis 4.9. Comparison 4 Fluoroquinolones vs azithromycin, Outcome 9 Microbiological failure (mostly children). 100

Analysis 4.10. Comparison 4 Fluoroquinolones vs azithromycin, Outcome 10 Relapse (mostly children). . . . . 100

Analysis 4.11. Comparison 4 Fluoroquinolones vs azithromycin, Outcome 11 Fever clearance time (mostly children). 101

Analysis 4.12. Comparison 4 Fluoroquinolones vs azithromycin, Outcome 12 Length of hospital stay (mostly children). 101

Fluoroquinolones for treating typhoid and paratyphoid fever (enteric fever) (Review)

Copyright $\odot 2008$ The Cochrane Collaboration. Published by John Wiley \& Sons, Ltd.

(1)

(1)

4

5

(1)


Analysis 4.13. Comparison 4 Fluoroquinolones vs azithromycin, Outcome 13 Convalescent faecal carriage (mostly children). . . . . . . . . . . . . . . . . . . . . . . . . . . . . . . . . . . . . Analysis 4.14. Comparison 4 Fluoroquinolones vs azithromycin, Outcome 14 Complications (mostly children). . . Analysis 4.15. Comparison 4 Fluoroquinolones vs azithromycin, Outcome 15 Adverse events (not serious) (mostly children). . . . . . . . . . . . . . . . . . . . . . . . . . . . . . . . . . . . .

Analysis 5.1. Comparison 5 Fluoroquinolones vs cefixime, Outcome 1 Clinical failure (adults or mostly adults). . . Analysis 5.2. Comparison 5 Fluoroquinolones vs cefixime, Outcome 2 Microbiological failure (adults or mostly adults). Analysis 5.3. Comparison 5 Fluoroquinolones vs cefixime, Outcome 3 Relapse (adults or mostly adults). . . . . . Analysis 5.4. Comparison 5 Fluoroquinolones vs cefixime, Outcome 4 Fever clearance time (adults or mostly adults). Analysis 5.5. Comparison 5 Fluoroquinolones vs cefixime, Outcome 5 Convalescent faecal carriage (adults or mostly adults).

Analysis 5.6. Comparison 5 Fluoroquinolones vs cefixime, Outcome 6 Complications (adults or mostly adults). . . . Analysis 5.7. Comparison 5 Fluoroquinolones vs cefixime, Outcome 7 Adverse events (not serious) (adults or mostly adults). . . . . . . . . . . . . . . . . . . . . . . . . . . . . . . . . . . . . . . . . . . .

Analysis 5.8. Comparison 5 Fluoroquinolones vs cefixime, Outcome 8 Clinical failure (children only). . . . . . Analysis 5.9. Comparison 5 Fluoroquinolones vs cefixime, Outcome 9 Microbiological failure (children only). . . . . Analysis 5.10. Comparison 5 Fluoroquinolones vs cefixime, Outcome 10 Relapse (children only). . . . . . . . Analysis 5.11. Comparison 5 Fluoroquinolones vs cefixime, Outcome 11 Fever clearance time (children only). . . Analysis 5.12. Comparison 5 Fluoroquinolones vs cefixime, Outcome 12 Length of hospital stay (children only). . . Analysis 5.13. Comparison 5 Fluoroquinolones vs cefixime, Outcome 13 Complications (children only). . . . . Analysis 6.1. Comparison 6 Fluoroquinolones vs ceftriaxone, Outcome 1 Clinical failure. . . . . . . . . . . . . Analysis 6.2. Comparison 6 Fluoroquinolones vs ceftriaxone, Outcome 2 Microbiological failure. . . . . . . . . . Analysis 6.3. Comparison 6 Fluoroquinolones vs ceftriaxone, Outcome 3 Relapse. . . . . . . . . . . . . . . . Analysis 6.4. Comparison 6 Fluoroquinolones vs ceftriaxone, Outcome 4 Fever clearance time. . . . . . . . . . . . Analysis 6.5. Comparison 6 Fluoroquinolones vs ceftriaxone, Outcome 5 Convalescent faecal carriage. . . . . . . Analysis 6.6. Comparison 6 Fluoroquinolones vs ceftriaxone, Outcome 6 Complications. . . . . . . . . . . . . . . Analysis 6.7. Comparison 6 Fluoroquinolones vs ceftriaxone, Outcome 7 Serious adverse events. . . . . . . . . . Analysis 6.8. Comparison 6 Fluoroquinolones vs ceftriaxone, Outcome 8 Adverse events (not serious). . . . . . . Analysis 7.1. Comparison 7 Norfloxacin vs chloramphenicol, Outcome 1 Clinical failure. . . . . . . . . . . Analysis 7.2. Comparison 7 Norfloxacin vs chloramphenicol, Outcome 2 Microbiological failure. . . . . . . . Analysis 7.3. Comparison 7 Norfloxacin vs chloramphenicol, Outcome 3 Relapse. . . . . . . . . . . . . . Analysis 7.4. Comparison 7 Norfloxacin vs chloramphenicol, Outcome 4 Fever clearance time. . . . . . . . . . . Analysis 7.5. Comparison 7 Norfloxacin vs chloramphenicol, Outcome 5 Length of hospital stay (MDR and NaR present). . . . . . . . . . . . . . . . . . . . . . . . . . . . . . . . . . . . Analysis 7.6. Comparison 7 Norfloxacin vs chloramphenicol, Outcome 6 Convalescent faecal carriage. . . . . . Analysis 7.7. Comparison 7 Norfloxacin vs chloramphenicol, Outcome 7 Adverse events (not serious). . . . . . Analysis 8.1. Comparison 8 Norfloxacin vs ceftriaxone, Outcome 1 Clinical failure (NaR not reported). . . . . . . Analysis 8.2. Comparison 8 Norfloxacin vs ceftriaxone, Outcome 2 Relapse (NaR not reported). . . . . . . . . . Analysis 8.3. Comparison 8 Norfloxacin vs ceftriaxone, Outcome 3 Fever clearance time (NaR not reported). . . . Analysis 8.4. Comparison 8 Norfloxacin vs ceftriaxone, Outcome 4 Adverse events (not serious) (NaR not reported). Analysis 9.1. Comparison 9 Norfloxacin vs other fluoroquinolones (FQ), Outcome 1 Clinical failure. . . . . . . Analysis 9.2. Comparison 9 Norfloxacin vs other fluoroquinolones (FQ), Outcome 2 Relapse. . . . . . . . . . . . Analysis 9.3. Comparison 9 Norfloxacin vs other fluoroquinolones (FQ), Outcome 3 Fever clearance time. . . . . . Analysis 9.4. Comparison 9 Norfloxacin vs other fluoroquinolones (FQ), Outcome 4 Convalescent faecal carriage. . Analysis 9.5. Comparison 9 Norfloxacin vs other fluoroquinolones (FQ), Outcome 5 Adverse events (not serious). . Analysis 10.1. Comparison 10 Fluoroquinolones for 2 days vs 3 days, Outcome 1 Clinical failure (NaR present). . . Analysis 10.2. Comparison 10 Fluoroquinolones for 2 days vs 3 days, Outcome 2 Microbiological failure (NaR present). Analysis 10.3. Comparison 10 Fluoroquinolones for 2 days vs 3 days, Outcome 3 Relapse (NaR present). . . . . Analysis 10.4. Comparison 10 Fluoroquinolones for 2 days vs 3 days, Outcome 4 Fever clearance time (NaR present). Analysis 10.5. Comparison 10 Fluoroquinolones for 2 days vs 3 days, Outcome 5 Length of hospital stay (NaR present). Analysis 10.6. Comparison 10 Fluoroquinolones for 2 days vs 3 days, Outcome 6 Convalescent faecal carriage (NaR present). . . . . . . . . . . . . . . . . . . . . . . . . . . . . . . . . . . . . . . . . . . . . . . . Analysis 10.7. Comparison 10 Fluoroquinolones for 2 days vs 3 days, Outcome 7 Complications (NaR present). . . 
Analysis 10.8. Comparison 10 Fluoroquinolones for 2 days vs 3 days, Outcome 8 Adverse events (not serious) (NaR present). . . . . . . . . . . . . . . . . . . . . . . . . . . . . . . . . . . .

Analysis 11.1. Comparison 11 Fluoroquinolones for 3 days vs 5 days, Outcome 1 Clinical failure (NaR not reported).

Analysis 11.2. Comparison 11 Fluoroquinolones for 3 days vs 5 days, Outcome 2 Relapse. . . . . . . . . . . .

Analysis 11.3. Comparison 11 Fluoroquinolones for 3 days vs 5 days, Outcome 3 Fever clearance time. . . . . .

Analysis 11.4. Comparison 11 Fluoroquinolones for 3 days vs 5 days, Outcome 4 Length of hospital stay (NaR not reported).

Analysis 11.5. Comparison 11 Fluoroquinolones for 3 days vs 5 days, Outcome 5 Adverse events (not serious) (NaR present).

Analysis 12.1. Comparison 12 Fluoroquinolones for 5 days vs 7 days, Outcome 1 Microbiological failure (NaR not reported). . . . . . . . . . . . . . . . . . . . . . . . . . . . . . . . . . . .

Analysis 12.2. Comparison 12 Fluoroquinolones for 5 days vs 7 days, Outcome 2 Relapse (NaR not reported). .

Analysis 12.3. Comparison 12 Fluoroquinolones for 5 days vs 7 days, Outcome 3 Fever clearance time (NaR not reported).

Analysis 12.4. Comparison 12 Fluoroquinolones for 5 days vs 7 days, Outcome 4 Adverse events (not serious) ( $\mathrm{NaR}$ not reported). . . . . . . . . . . . . . . . . . . . . . . . . . . . . . . . . . . .

Analysis 13.1. Comparison 13 Fluoroquinolones for 7 days vs 10 or 14 days, Outcome 1 Microbiological failure (NaR not reported). . . . . . . . . . . . . . . . . . . . . . . . . . . . . . . . . . . . 139

Analysis 13.2. Comparison 13 Fluoroquinolones for 7 days vs 10 or 14 days, Outcome 2 Relapse (NaR not reported). 140

Analysis 14.1. Comparison 14 Fluoroquinolones for 10 days vs 14 days, Outcome 1 Relapse (NaR present). $\quad$. . $\quad 140$

Analysis 14.2. Comparison 14 Fluoroquinolones for 10 days vs 14 days, Outcome 2 Fever clearance time (NaR present). 141

Analysis 14.3. Comparison 14 Fluoroquinolones for 10 days vs 14 days, Outcome 3 Adverse events (not serious) (NaR present). . . . . . . . . . . . . . . . . . . . . . . . . . . . . . . . . . . . . . 141

WHAT'S NEW . . . . . . . . . . . . . . . . . . . . . . . . . . . . . . . . . . . . . 141

HISTORY . . . . . . . . . . . . . . . . . . . . . . . . . . . . . . . . . . . . . . . 159

CONTRIBUTIONS OF AUTHORS . . . . . . . . . . . . . . . . . . . . . . . . . . . . . . . . . . . . . . . 160

DECLARATIONS OF INTEREST . . . . . . . . . . . . . . . . . . . . . . . . . . . . . . . . . . . . . . . . .

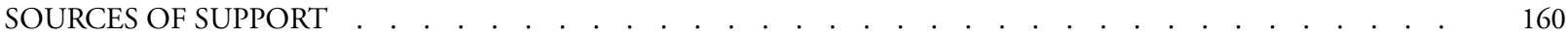

DIFFERENCES BETWEEN PROTOCOL AND REVIEW . . . . . . . . . . . . . . . . . . . . . . 160

INDEX TERMS . . . . . . . . . . . . . . . . . . . . . . . . . . . . . . . . . . . . 160

Fluoroquinolones for treating typhoid and paratyphoid fever (enteric fever) (Review) 


\title{
Fluoroquinolones for treating typhoid and paratyphoid fever (enteric fever)
}

\author{
Durrane Thaver ${ }^{1}$, Anita KM Zaidi ${ }^{1}$, Julia A Critchley ${ }^{2}$, Asma Azmatullah ${ }^{1}$, Syed Ali Madni ${ }^{3}, Z_{\text {Zulfiqar A Bhutta }}{ }^{1}$ \\ ${ }^{1}$ Department of Paediatrics \& Child Health, The Aga Khan University Hospital, Karachi, Pakistan. ${ }^{2}$ Institute of Health and Society, \\ Newcastle University, Newcastle, UK. ${ }^{3}$ Department of Internal Medicine, Guthrie-Robert Packer Hospital, Sayre, Pennsylvania, USA \\ Contact address: Zulfiqar A Bhutta, Department of Paediatrics \& Child Health, The Aga Khan University Hospital, Stadium Road, \\ PO Box 3500, Karachi, 74800, Pakistan. zulfiqar.bhutta@aku.edu.
}

Editorial group: Cochrane Infectious Diseases Group.

Publication status and date: New search for studies and content updated (no change to conclusions), published in Issue 4, 2008. Review content assessed as up-to-date: 1 April 2008.

Citation: Thaver D, Zaidi AKM, Critchley JA, Azmatullah A, Madni SA, Bhutta ZA. Fluoroquinolones for treating typhoid and paratyphoid fever (enteric fever). Cochrane Database of Systematic Reviews 2008, Issue 4. Art. No.: CD004530. DOI: 10.1002/14651858.CD004530.pub3.

Copyright (C) 2008 The Cochrane Collaboration. Published by John Wiley \& Sons, Ltd.

\begin{abstract}
A B S T R A C T

\section{Background}

Fluoroquinolones are recommended as first-line therapy for typhoid and paratyphoid fever (enteric fever), but how they compare with other antibiotics and different fluoroquinolones is unclear.
\end{abstract}

\section{Objectives}

To evaluate fluoroquinolone antibiotics for treating enteric fever in children and adults compared with other antibiotics, different fluoroquinolones, and different durations of fluoroquinolone treatment.

\section{Search strategy}

In November 2007, we searched the Cochrane Infectious Diseases Group Specialized Register, CENTRAL (The Cochrane Library 2007, Issue 4), MEDLINE, EMBASE, LILACS, $m \mathrm{RCT}$, conference proceedings, and reference lists.

\section{Selection criteria}

Randomized controlled trials of fluoroquinolones in people with blood or bone marrow culture-confirmed enteric fever.

\section{Data collection and analysis}

Two authors independently assessed the trials' methodological quality and extracted data. We calculated odds ratios (OR) for dichotomous data with $95 \%$ confidence intervals (CI). We analysed trials with greater than $60 \%$ children separately from trials of mostly adults.

\section{Main results}

Of 38 included trials, 22 had unclear allocation concealment and 34 did not use blinding. Four trials included exclusively children, seven had both adults and children, and three studied outpatients. ADULTS: Among primary outcomes (clinical failure, microbiological failure, and relapse), compared with chloramphenicol, fluoroquinolones were not statistically significantly different for clinical failure (594 participants) or microbiological failure (378 participants), but they reduced clinical relapse (OR 0.14, 95\% CI 0.04 to 0.50 ; 467 participants, 6 trials). We detected no statistically significant difference versus co-trimoxazole (82 participants, 2 trials) or azithromycin

Fluoroquinolones for treating typhoid and paratyphoid fever (enteric fever) (Review)

Copyright @ 2008 The Cochrane Collaboration. Published by John Wiley \& Sons, Ltd. 
(152 participants, 2 trials). Fluoroquinolones reduced clinical failure compared with ceftriaxone (OR 0.08, 95\% CI 0.01 to $0.45 ; 120$ participants, 3 trials), but not microbiological failure or relapse. Versus cefixime, fluoroquinolones reduced clinical failure (OR 0.05 , 95\% CI 0.01 to $0.24 ; 238$ participants; 2 trials) and relapse (OR 0.18, 95\% CI 0.03 to $0.91 ; 218$ participants, 2 trials). CHILDREN: In children with high proportions of nalidixic acid-resistant strains, older fluoroquinolones increased clinical failures compared with azithromycin (OR 2.67, 95\% CI 1.16 to $6.11 ; 125$ participants, 1 trial), with no differences using newer fluoroquinolones (285 participants, 1 trial). Fluoroquinolones and cefixime were not statistically significantly different ( 82 participants, 1 trial). Trials comparing different durations of fluoroquinolone treatment were not statistically significantly different (889 participants, 9 trials). Norfloxacin had more clinical failures than other fluoroquinolones (417 participants, 5 trials).

\section{Authors' conclusions}

Trials were small and methodological quality varied. In adults, fluoroquinolones may be better for reducing clinical relapse rates compared to chloramphenicol. Data are limited for other comparisons, particularly in children.

\section{PLAIN LANGUAGE SUMMARY}

\section{Not enough sound evidence for using fluoroquinolones in typhoid and paratyphoid fever compared with the standard antibiotics}

The potentially fatal typhoid and paratyphoid fevers are caused by bacterial infection that begins in the small intestine (enteric fever). Transmission occurs through contaminated food and water, and there are areas where these diseases are endemic, such as Asia, Africa, and South and Central America. People often relapse or become carriers. Chloramphenicol has been the standard treatment, but the bacteria are becoming resistant. A new group of drugs, the fluoroquinolones, are being tried, but the review of trials found there were insufficient numbers of participants in the trials, which were also of varying quality, to be able to give recommendations with any degree of certainty, especially for children.

\section{B A C K G R O U N D}

\section{Definition}

Enteric fever refers to either typhoid or paratyphoid fever. Typhoid fever is caused by Salmonella enterica serovar Typhi (S. Typhi), an enteric bacterium that colonizes only human hosts. Since humans are the only natural hosts of $S$. Typhi, direct or indirect contact with someone with typhoid fever or who is carrying $S$. Typhi (without symptoms) is essential for transmission of infection (Cleary 2000). Transmission most commonly occurs when susceptible individuals ingest food or water contaminated with faeces harbouring $S$. Typhi. Paratyphoid fever is considered a similar, but generally milder illness, and is caused by $S$. enterica serovar Paratyphi (S. Paratyphi) A, B, or C (Lee 2000). A recent report, however, suggests that the illness caused by $S$. Paratyphi A may be equal in severity to typhoid fever (Maskey 2006).

\section{Epidemiology}

Enteric fever - the majority of cases of which are caused by $S$. Typhi - continues to be a major health problem due to poor hygienic and sanitary conditions prevalent in low-income and middle-income countries. However, the pattern of enteric fever is changing in some endemic areas with an increase in the relative frequency of $S$. Paratyphi A isolated from patients with enteric fever (Chandel 2000; Ahmad 2002; Butt 2005; Ochiai 2005; Jesudason 2005; Woods 2006; Maskey 2008). Each year there are an estimated 16 million cases of enteric fever caused by $S$. Typhi and about 600,000 deaths (Ivanoff 1995). According to recent estimates the burden of typhoid for the year 2000 was 21 million cases (Crump 2004). Endemic regions comprise almost all of Asia (with South and South-East Asia considered areas of high incidence, ie over 100 cases per 100,000 population per year), Middle East, Africa, and South and Central America (Ivanoff 1995; Crump 2004). In population-based studies from endemic areas, the highest incidence has been reported in children between five and 10 years of age (Lin 
2000; Siddiqui 2006; Sur 2006) as well as in children under five years of age (Sinha 1999; Saha 2001; Saha 2003; Brooks 2005). In high-income countries, enteric fever has been virtually eliminated and most cases are those occurring in travellers returning from endemic areas. In the USA, 2445 cases of infections caused by $S$. Typhi were reported to the Centers for Disease Control and Prevention between 1985 and 1994, 72\% of which were associated with international travel mainly to Mexico and the Indian subcontinent (Mermin 1998).

In endemic areas, most people are treated as outpatients, thus hospital-based data represent a subset of patients with a more severe illness who may consequently have a less favourable response to conventional therapy.

\section{Pathogenesis}

On ingestion, the salmonellae invade the intestinal epithelium, probably through the Peyer's patches (Cleary 2000). They then multiply in the lymphoid tissue, enter the mesenteric lymph nodes, and eventually reach the bloodstream. Once in the bloodstream, which is referred to as the 'primary blood stream invasion', the bacteria seed and multiply in several reticuloendothelial sites. The bacteria then spill over from these primarily infected sites back into the bloodstream, referred to as the 'secondary bloodstream invasion', and the patient begins to exhibit symptoms (Richens 2000). Infection then disseminates to several sites, most commonly the liver, spleen, bone marrow, gall bladder, and Peyer's patches (Lee 2000; Richens 2000). The ability of $S$. Typhi and $S$. Paratyphi to survive within macrophages is considered essential to the pathogenesis of enteric fever (Miller 2000).

\section{Clinical features and diagnosis}

The clinical features of enteric fever are non-specific and vary in different populations (Parry 2002). Common symptoms include fever, headache, and gastrointestinal complaints, such as diarrhoea or constipation, abdominal pain, nausea and vomiting, or loss of appetite (Lee 2000; Richens 2000). Some common findings on examination include liver enlargement, spleen enlargement, a coated tongue, and abdominal tenderness (Lee 2000; Richens 2000). The definitive diagnosis of enteric fever requires the isolation of $S$. Typhi or $S$. Paratyphi from blood, bone marrow, urine, bile, rose spots, and gastric or intestinal secretions. Blood cultures have a sensitivity of $60 \%$ to $80 \%$, while bone marrow cultures have a greater sensitivity of $80 \%$ to $95 \%$ (Parry 2002).

\section{Prognosis}

About $10 \%$ of people infected with $S$. Typhi experience a relapse in the absence of treatment (Richens 2000). About $1 \%$ to $4 \%$ of people become long-term carriers (Lee 2000). Case-fatality rates range widely, from $1.6 \%$ in a hospital in Pakistan (Bhutta 1996) to as high as $44 \%$ in a subgroup of people with severe $S$. Typhi infection in Papua New Guinea (Richens 1995). The greater number of deaths observed in some low-income and middle-income countries could be due to delays in diagnosis, hospitalization, and commencement of effective treatment.

\section{Treatment}

Since its introduction in 1948, chloramphenicol has been widely used for treating enteric fever because of its wide availability and low cost. But chloramphenicol has some major disadvantages: it does not reduce the relapse rate (the rate of recurrence of infection with symptoms); it has no effect on the convalescent carrier or chronic carrier (a person who continues to excrete the organism in stool for one year after the illness); and it is not useful for treating multiple-drug-resistant (MDR) S. Typhi (Lee 2000).

MDR strains of $S$. Typhi, carrying plasmid-encoded resistance to all conventional first-line antibiotics (chloramphenicol, co-trimoxazole, and ampicillin or amoxicillin), have become highly prevalent in several areas of the world since 1989. In the Indian subcontinent and China, the frequency of these MDR strains ranged from $50 \%$ to $80 \%$ of all $S$. Typhi isolates and reached $100 \%$ during outbreaks (Lee 2000). Effective treatment for people infected with MDR strains is critical because they have been observed to have a significantly higher incidence of complications than people infected with fully sensitive strains (Bhutta 1996). Many areas reported lower rates of MDR strains, and the re-emergence in some areas of strains fully susceptible to first-line antibiotics suggests that chloramphenicol could still be a valuable treatment option for enteric fever (Takkar 1995; Sood 1999; Wasfy 2002; Rodrigues 2003; Butt 2005; Walia 2005; Mohanty 2006). Conversely, recent studies have reported the emergence of MDR strains of S. Paratyphi A (Chandel 2000; Mahmood 2000; Butt 2005; Mohanty 2006).

The fluoroquinolones and other second-line antibiotics, such as third-generation cephalosporins (eg ceftriaxone and cefixime) and azithromycin (a macrolide antibiotic), are currently regarded as the antibiotics of choice for treating MDR strains.

\section{The fluoroquinolones}

The fluoroquinolones, such as ciprofloxacin, ofloxacin, fleroxacin, enoxacin, and pefloxacin, are a large family of anti-infective drugs synthesized around the quinolone core and that possess a broad antibacterial spectrum (Congeni 2002). The fluoroquinolones effectively penetrate macrophages and achieve high concentrations in bile (Miller 2000). Norfloxacin is the exception, because the World Health Organization (WHO) does not recommend it for 
treating enteric fever due to its low bioavailability (WHO 2003), which is 50\% compared with 95\% for ofloxacin (Hooper 2000). The fluoroquinolones are generally contraindicated for use in children under the age of 18 years, except for the treatment of certain infections, when no alternate agent is available (Gendrel 2003; Committee 2006). This contraindication is primarily due to concerns regarding their potential to cause arthropathy (joint disease), which has been clearly established in juvenile animal experiments (Simonin 1999). A review, however, reported that numerous studies evaluating ciprofloxacin use in children and adults have consistently failed to demonstrate cartilage damage (Congeni 2002). Arthralgia (joint pain) has been reported with fluoroquinolone use, but it occurs at a rate of less than $1.5 \%$ and appears to resolve entirely on discontinuation of the drug without leaving any evidence of long-term damage (Fish 2001).

The most common adverse effects associated with fluoroquinolones are gastrointestinal, such as nausea and diarrhoea, and central nervous system effects, such as headache, dizziness, and drowsiness (Fish 2001). Severe central nervous system events, such as psychosis and seizures are rare (Cross 2001). Other adverse effects include dermatologic reactions, hepatic enzyme elevation, hypersensitivity, nephrotoxicity, haematological reactions, tendonitis, and tendon rupture. Tendon rupture can occur with short-term use and small doses (Cross 2001). A potentially serious adverse effect is the prolongation of the QTc interval (Congeni 2002), which can lead to cardiac arrhythmias.

A summary of randomized controlled trials has shown that fluoroquinolones, when compared with ceftriaxone, cefixime, and firstline antibiotics, have lower clinical failure rates and lower fever clearance times in the treatment of enteric fever (Parry 2002). However, the review combined the data for both adults and children, and more importantly, the results for drug-sensitive and drug-resistant strains of $S$. Typhi and $S$. Paratyphi. The review also does not present findings of unpublished trials and may under represent non-English language trials, both of which are considered important components of a systematic review (Davies 1998).

The optimal duration of treatment for fluoroquinolones in enteric fever has yet to be clearly established. Although the recommended duration is 10 to 14 days, recent randomized controlled trials in Vietnam suggest that two-day and three-day courses may be sufficient to treat uncomplicated $S$. Typhi infections in children and adults (Tran 1995; Vinh 1996; Nguyen 1997). Such shortcourse therapy is favourable, as it will prove less costly, possibly less toxic, and will increase adherence to treatment.

However, a fact of great concern is the emergence of strains of $S$. Typhi and $S$. Paratyphi with reduced susceptibility to fluoroquinolones (Murdoch 1998; Threlfall 2001; Threlfall 2003; Rodrigues 2003; Karunanayake 2004; Slinger 2004; Butt 2005; Manchanda 2006; Mohanty 2006; Walia 2006; Chau 2007; Joshi 2007). This is demonstrated with the minimum inhibitory concentration (MIC) values for ciprofloxacin, which are higher $(0.125$ to $1 \mathrm{mg} / \mathrm{L}$ ) compared with the usual values for fully suscepti- ble strains $(<0.125 \mathrm{mg} / \mathrm{L}$ ) (Parry 2004). Increasing numbers of treatment failures in infections caused by such strains are being reported, with short as well as long durations of fluoroquinolones (Brown 1994; Wain 1997; Asna 2003; Butt 2003; Rupali 2004; Slinger 2004; Manchanda 2006; Dimitrov 2007). These strains often display resistance to nalidixic acid (a first-generation quinolone) on routine disk diffusion susceptibility testing. Thus the presence of nalidixic acid resistance $(\mathrm{NaR})$ among $S$. Typhi and $S$. Paratyphi can be used to identify strains with reduced susceptibility to fluoroquinolones (Wain 1997; Parry 2004), and is also the rationale for using $\mathrm{NaR}$ to denote reduced susceptibility to fluoroquinolones in this review. However, some strains with reduced susceptibility to fluoroquinolones do not exhibit $\mathrm{NaR}$ ( Threlfall 2003; Cooke 2006), which suggests the need, in future, for direct determination and interpretation of fluoroquinolone MICs. More concerning are the emerging reports of isolates with absolute fluoroquinolone resistance (Harish 2004; Adachi 2005; Renuka 2005; Ahmed 2006; Mohanty 2006; Walia 2006; Joshi 2007). Newer generation fluoroquinolones, such as gatifloxacin, however, have been found to be active against $\mathrm{NaR}$ strains (Pandit 2007; Dolecek 2008).

\section{O B J E C T I VES}

To evaluate fluoroquinolone antibiotics for treating enteric fever in children and adults compared with other antibiotics, different fluoroquinolones, and different durations of fluoroquinolone treatment.

\section{METHODS}

\section{Criteria for considering studies for this review}

\section{Types of studies}

Randomized controlled trials.

\section{Types of participants}

People diagnosed with typhoid or paratyphoid fever based on microbiological confirmation from blood or bone marrow.

\section{Types of interventions}

\section{Intervention}

Fluoroquinolone antibiotic. 
Control

- Non-fluoroquinolone antibiotic (one or more).

- Different fluoroquinolone antibiotic.

- Different treatment duration of same fluoroquinolone antibiotic.

\section{Types of outcome measures}

\section{Primary}

- Clinical failure, defined as the presence of symptoms or the development of complications that necessitate change in antibiotic therapy or prolongation of existing therapy at the period specified by trial authors.

- Microbiological failure, defined as a positive culture from blood, bone marrow, or any sterile anatomic site at the period specified by trial authors.

- Relapse, defined as the recurrence of symptoms with a positive culture from blood or bone marrow or any sterile anatomic site, to the point of follow up defined by trial authors.

\section{Secondary}

- Fever clearance time, defined as the time in hours taken to defervesce from the start of the intervention or control drug with the definition of fever clearance as specified by trial authors.

- Length of hospital stay, defined as the time in days from entry into trial until discharge.

- Cost of therapy, defined as the total cost in US\$ of the drug, drug delivery, and hospital stay.

- Convalescent faecal carriage, defined as a positive faecal culture detected at any time after the end of treatment up to one year of follow up.

\section{Complications and adverse events (as defined by trial authors)}

- Complications, defined as the appearance of complications during therapy such as abdominal (intestinal perforation, intestinal haemorrhage, hepatitis), cardiovascular (myocarditis, shock), neuropsychiatric (delirium, meningitis), respiratory (pneumonia, bronchitis), or haematological (anaemia, disseminated intravascular coagulation).

- Serious adverse events, defined as adverse events leading to death, requiring inpatient hospitalization or prolonged existing hospitalization, or life threatening, or resulting in persistent or significant disability or incapacity, such as joint disease, tendonitis and tendon rupture, prolongation of QTc interval, seizures, nephrotoxicity, haematological reactions, or severe dermatologic reactions.

- Other adverse events, such as nausea, diarrhoea, headache, dizziness, mild photosensitivity, hepatic enzyme elevations, and hypersensitivity reactions.

\section{Search methods for identification of studies}

Durrane Thaver worked with Vittoria Lutje (Information Retrieval Specialist, Cochrane Infectious Diseases Group) to attempt to identify all relevant trials regardless of language or publication status.

\section{Databases}

We searched the following databases using the search terms and strategy described in Table 4: Cochrane Infectious Diseases Group Specialized Register (November 2007); Cochrane Central Register of Controlled Trials (CENTRAL), published in The Cochrane Library (2007, Issue 4); MEDLINE (1966 to November 2007); EMBASE (1974 to November 2007); and LILACS (1982 to November 2007). We also searched the metaRegister of Controlled Trials $(m$ RCT) in November 2007 using the search term "(typhoid fever) NOT vaccine".

Table 4. Detailed search strategies

\begin{tabular}{llllll}
\hline Search set & CIDG SR $^{a}$ & CENTRAL & MEDLINE $^{b}$ & EMBASE $^{b}$ & LILACS $^{b}$ \\
\hline 1 & typhoid fever & fluoroquinolone & QUINOLINES & $\begin{array}{l}\text { QUINOLONE } \\
\text { DERIVED ANTIIN- } \\
\text { FECTIVE AGENT }\end{array}$ \\
\hline 2 & enteric fever & amifloxacin & QUINOLONES & fluoroquinolones & typhoid fever
\end{tabular}

Fluoroquinolones for treating typhoid and paratyphoid fever (enteric fever) (Review) 
Table 4. Detailed search strategies (Continued)

\begin{tabular}{|c|c|c|c|c|c|}
\hline 3 & paratyphoid fever & balofloxacin & $\begin{array}{l}\text { ANTI-IN- } \\
\text { FECTIVE AGENTS, } \\
\text { QUINOLONE }\end{array}$ & amifloxacin & enteric fever \\
\hline 4 & Salmonella typhi & cetefloxacin & $\begin{array}{l}\text { ANTI-INFECTIVE } \\
\text { AGENTS, FLUO- } \\
\text { ROQUINOLONE }\end{array}$ & BALOFLOXACIN & Salmonella typhi \\
\hline 5 & Salmonella paratyphi & ciprofloxacin & $\begin{array}{l}\text { FLUORO- } \\
\text { QUINOLONES }\end{array}$ & balofloxacin & Salmonella paratyphi \\
\hline 6 & - & clinafloxacin & fluoroquinolones & CETEFLOXACIN & - \\
\hline 7 & - & enoxacin & amifloxacin & cetefloxacin & - \\
\hline 8 & - & fleroxacin & balofloxacin & CIPROFLOXACIN & - \\
\hline 9 & - & gatifloxacin & cetefloxacin & ciprofloxacin & - \\
\hline 10 & - & gemifloxacin & CIPROFLOXACIN & CLINAFLOXACIN & - \\
\hline 11 & - & grepafloxacin & ciprofloxacin & clinafloxacin & - \\
\hline 12 & - & irloxacin & clinafloxacin & ENOXACIN & - \\
\hline 13 & - & levofloxacin & ENOXACIN & enoxacin & - \\
\hline 14 & - & lomefloxacin & enoxacin & FLEROXACIN & - \\
\hline 15 & - & moxifloxacin & FLEROXACIN & fleroxacin & - \\
\hline 16 & - & nordifloxacin & fleroxacin & GATIFLOXACIN & - \\
\hline 17 & - & norfleroxacin & gatifloxacin & gatifloxacin & - \\
\hline 18 & - & norfloxacin & gemifloxacin & GEMIFLOXACIN & - \\
\hline 19 & - & ofloxacin & grepafloxacin & gemifloxacin & - \\
\hline 20 & - & oxociprofloxacin & irloxacin & GREPAFLOXACIN & - \\
\hline 21 & - & pefloxacin & levofloxacin & grepafloxacin & - \\
\hline 22 & - & premafloxacin & lomefloxacin & IRLOXACIN & - \\
\hline 23 & - & prulifloxacin & moxifloxacin & irloxacin & - \\
\hline 24 & - & rufloxacin & nordifloxacin & LEVOFLOXACIN & - \\
\hline
\end{tabular}


Table 4. Detailed search strategies (Continued)

\begin{tabular}{|c|c|c|c|c|c|}
\hline 25 & - & sitafloxacin & norfleroxacin & levofloxacin & - \\
\hline 26 & - & sparfloxacin & NORFLOXACIN & LOMEFLOXACIN & - \\
\hline 27 & - & temafloxacin & norfloxacin & lomefloxacin & - \\
\hline 28 & - & tosufloxacin & ofloxacin & MOXIFLOXACIN & - \\
\hline 29 & - & trovafloxacin & oxociprofloxacin & moxifloxacin & - \\
\hline 30 & - & $1 / 29-\mathrm{OR}$ & PEFLOXACIN & NORDIFLOXACIN & - \\
\hline 31 & - & typhoid fever & pefloxacin & nordifloxacin & - \\
\hline 32 & - & enteric fever & premafloxacin & NORFLEROXACIN & - \\
\hline 33 & - & paratyphoid fever & prulifloxacin & norfleroxacin & - \\
\hline 34 & - & Salmonella typhi & rufloxacin & NORFLOXACIN & - \\
\hline 35 & - & Salmonella paratyphi & sitafloxacin & norfloxacin & - \\
\hline 36 & - & $31 / 35-$ OR & sparfloxacin & OFLOXACIN & - \\
\hline 37 & - & 30 and 36 & temafloxacin & ofloxacin & - \\
\hline 38 & - & - & tosufloxacin & $\begin{array}{l}\text { OXO- } \\
\text { CIPROFLOXACIN }\end{array}$ & - \\
\hline 39 & - & - & trovafloxacin & oxociprofloxacin & - \\
\hline 40 & - & - & $1-39 / \mathrm{OR}$ & PEFLOXACIN & - \\
\hline 41 & - & - & TYPHOID FEVER & pefloxacin & - \\
\hline 42 & - & - & typhoid fever & PREMAFLOXACIN & - \\
\hline 43 & - & - & enteric fever & premafloxacin & - \\
\hline 44 & - & - & $\begin{array}{l}\text { PARATYPHOID } \\
\text { FEVER }\end{array}$ & PRULIFLOXACIN & - \\
\hline 45 & - & - & paratyphoid fever & prulifloxacin & - \\
\hline 46 & - & - & $\begin{array}{l}\text { SALMONELLA TY- } \\
\text { PHI }\end{array}$ & RUFLOXACIN & - \\
\hline 47 & - & - & Salmonella typhi & rufloxacin & - \\
\hline
\end{tabular}


Table 4. Detailed search strategies (Continued)

\begin{tabular}{|c|c|c|c|c|c|}
\hline 48 & - & - & $\begin{array}{l}\text { SALMONELLA } \\
\text { PARATYPHI }\end{array}$ & SITAFLOXACIN & - \\
\hline 49 & - & - & Salmonella paratyphi & sitafloxacin & - \\
\hline 50 & - & - & typhus & SPARFLOXACIN & - \\
\hline 51 & - & - & $41-50 / \mathrm{OR}$ & sparfloxacin & - \\
\hline 52 & - & - & 40 and 51 & TEMAFLOXACIN & - \\
\hline 53 & - & - & limit 52 to human & temafloxacin & - \\
\hline 54 & - & - & - & tosufloxacin & - \\
\hline 55 & - & - & - & $1-54 / \mathrm{OR}$ & - \\
\hline 56 & - & - & - & TYPHOID FEVER & - \\
\hline 57 & - & - & - & typhoid fever & - \\
\hline 58 & - & - & - & enteric fever & - \\
\hline 59 & - & - & - & $\begin{array}{l}\text { PARATYPHOID } \\
\text { FEVER }\end{array}$ & - \\
\hline 60 & - & - & - & paratyphoid fever & - \\
\hline 61 & - & - & - & $\begin{array}{l}\text { SALMONELLA TY- } \\
\text { PHI }\end{array}$ & - \\
\hline 62 & - & - & - & Salmonella typhi & - \\
\hline 63 & - & - & - & $\begin{array}{l}\text { SALMONELLA } \\
\text { PARATYPHI }\end{array}$ & - \\
\hline 64 & - & - & - & Salmonella paratyphi & - \\
\hline 65 & - & - & - & typhus & - \\
\hline 66 & - & - & - & $56-65 / \mathrm{OR}$ & - \\
\hline 67 & - & - & - & 55 and 66 & - \\
\hline 68 & - & - & - & limit 67 to human & - \\
\hline
\end{tabular}

${ }^{a}$ Cochrane Infectious Diseases Group Specialized Register.

${ }^{b}$ Search terms used in combination with the search strategy for retrieving trials developed by The Cochrane Collaboration (Higgins 2006); upper case: MeSH or EMTREE heading; lower case: free text term. 


\section{Conference proceedings}

We searched the following conference proceedings for relevant abstracts: $5^{\text {th }}$ International Symposium on Typhoid Fever and other Salmonelloses, Karachi, Pakistan, 4 to 7 February 2002; $8^{\text {th }}$ Western Pacific Congress of Chemotherapy and Infectious Diseases, Perth, Australia, 1 to 5 December 2002; $43^{\text {rd }}$ Interscience Conference on Antimicrobial Agents and Chemotherapy (ICAAC), 14 to 17 September 2003, Chicago, USA; Final Programs of the $44^{\text {th }}$ ICAAC, 30 October to 2 November 2004, in Washington D.C., USA; and the $45^{\text {th }}$ ICAAC, 16 to 19 December 2005, Washington D.C., USA.

\section{Researchers}

We contacted Dr Christopher Parry (in 2003) and Dr Jeremy Farrar and Dr Christiane Dolecek (in December 2007) for information on unpublished and ongoing trials.

\section{Reference lists and review authors' personal collections}

We also checked the reference lists of all retrieved trials and searched the review authors' personal literature collections for relevant trials.

\section{Data collection and analysis}

\section{Selection of studies}

Durrane Thaver and Asma Azmatullah screened the title, abstract, or keywords of each record identified with the search strategy, and retrieved the full text for potentially relevant trials and for records where the relevance was unclear. Durrane Thaver and Asma Azmatullah or Ali Madni independently applied the inclusion criteria to each potentially relevant trial to determine their eligibility. We resolved any disagreements through discussion with Anita Zaidi, or attempted to contact the trial authors if we still had doubts. We tabulated the excluded studies along with the reason for excluding them in the 'Characteristics of excluded studies'. We ensured that data from each trial was entered only once in our review.

\section{Data extraction and management}

Durrane Thaver and Asma Azmatullah or Ali Madni independently extracted data. For dichotomous outcomes, such as clinical failure, we extracted the total number of participants and number of participants that experienced the event. For continuous outcomes, such as fever clearance time, we extracted the total number of participants, arithmetic means, and standard deviations. If the standard deviation was not reported, we attempted to use the confidence interval or P value to derive it. We attempted to contact authors for means and standard deviations when they were not available. We also attempted to contact all trial authors to obtain additional data or when the data were not in the format we required. We compared the extracted data to identify errors.
We resolved disagreements by consulting Anita Zaidi (or Zulfiqar Bhutta). Durrane Thaver and Asma Azmatullah entered data into Review Manager 4.2.

\section{Assessment of risk of bias in included studies}

Durrane Thaver and Asma Azmatullah or Ali Madni assessed the methodological quality of each included trial by assessing generation of allocation sequence, allocation concealment, blinding, and loss to follow up. We assessed generation of allocation sequence and allocation concealment as adequate, inadequate, or unclear ( Jüni 2001). We described blinding as double (trial uses a placebo or a double dummy technique such that neither the participant or care provider/assessor know which treatment is given), single (participant or care provider/assessor is aware of the treatment given), or open (all parties are aware of treatment), and considered it adequate if $90 \%$ or more of the randomized culture-positive participants were in the final analysis and inadequate if less than $90 \%$. If the method was unclear, we attempted to contact the trial authors for clarification. We resolved disagreements through discussion and by consulting Anita Zaidi.

\section{Data synthesis}

We analysed data using Review Manager 4.2. We used the odds ratio (OR) for dichotomous data and the mean difference (MD) for continuous data, and presented each result with a $95 \%$ confidence interval (CI). We combined trials of different fluoroquinolones when evaluating treatment durations. We have not combined trials comparing: fluoroquinolones with different antibiotics (eg trials of fluoroquinolone versus chloramphenicol are not combined with trials of fluoroquinolone versus amoxicillin); adult participants with child participants; or drug-resistant $S$. Typhi and $S$. Paratyphi strains (NaR or MDR) with drug-sensitive $S$. Typhi and $S$. Paratyphi strains. We also analysed norfloxacin trials separate from the other fluoroquinolones because the WHO does not recommend this fluoroquinolone for treating enteric fever due to its low bioavailability (WHO 2003).

\section{Stratification}

We stratified the results according to the presence or absence of drug-resistant strains (MDR and $\mathrm{NaR}$ ). We defined MDR as resistant to all three first-line antibiotics (chloramphenicol, co-trimoxazole, and ampicillin or amoxicillin).

\section{- Fluoroquinolones versus first-line antibiotics}

(chloramphenicol, co-trimoxazole, and ampicillin or amoxicillin) and norfloxacin versus chloramphenicol: Since the presence of MDR would affect the performance of first-line antibiotics, we stratified the trials into those that reported the absence of MDR strains, reported their presence, or did not report them or were unavailable. We also stratified the trials by the reported absence, presence, or not reporting of $\mathrm{NaR}$ strains, since this would affect the performance of the fluoroquinolone arm. 
Since the proportion of MDR strains would not differentially affect the performance of the second-line or the fluoroquinolone antibiotics, we did not stratify the results by MDR strains for the subsequent comparisons.

- Fluoroquinolones versus second-line antibiotics (azithromycin, ceftriaxone, cefixime) and norfloxacin versus ceftriaxone: We stratified these trials by the reported absence, presence, or not reporting or testing of $\mathrm{NaR}$ strains.

- Norfloxacin versus other fluoroquinolones: We did not stratify these by the $\mathrm{NaR}$ strains since both arms involved fluoroquinolones.

- Different durations of fluoroquinolones: Since the efficacy of different durations of fluoroquinolones would be affected by presence of $\mathrm{NaR}$ strains, we stratified these by $\mathrm{NaR}$ strains as described above.

However, in all above-mentioned analyses of $\mathrm{NaR}$, in order to differentiate trials that had participants with $\mathrm{NaR}$ strains but involved newer fluoroquinolones which are not affected by $\mathrm{NaR}$, we made a separate category ("NaR present, but newer fluoroquinolone"). Further analyses with varying proportions strains with reduced susceptibility to fluoroquinolones may be possible in future updates of the review.

We also conducted separate analyses for those trials that included mainly (more than 60\%) children (defined as less than 16 years or as in text of trial) and those that included mainly adults; we classified all participants as adults if they were described as such by trial authors, regardless of the age of the youngest participant.

\section{Intention-to-treat analyses}

We were unable to conduct an intention-to-treat analysis on culture-positive cases since no further information was available for culture-positive participants who were lost to follow up. Instead, we conducted an available-case analysis, and we derived the per cent loss to follow up and tabulated the results (see Table 5).

Table 5. Assessment of risk of bias ${ }^{a}$

\begin{tabular}{|c|c|c|c|c|c|}
\hline Comparison & Trial & $\begin{array}{l}\text { Generation of allo- } \\
\text { cation sequence }\end{array}$ & $\begin{array}{l}\text { Allocation } \\
\text { concealment }\end{array}$ & Blinding & $\begin{array}{l}\text { Inclusion of all randomized } \\
\text { culture-positive participants } \\
\text { in the final analysis }\end{array}$ \\
\hline \multirow{4}{*}{$\begin{array}{l}\text { Fluoroquinolone vs } \\
\text { chloramphenicol }\end{array}$} & Abejar 1993 & Unclear & Unclear & Open & Adequate \\
\hline & Arnold 1993 & Unclear & Unclear & Open & Adequate \\
\hline & Cristiano 1995 & Adequate & Unclear & Open & Adequate \\
\hline & Bran 1991 & Unclear & Unclear & Double & Adequate \\
\hline
\end{tabular}

Fluoroquinolones for treating typhoid and paratyphoid fever (enteric fever) (Review) 
Table 5. Assessment of risk of bias ${ }^{a} \quad$ (Continued)

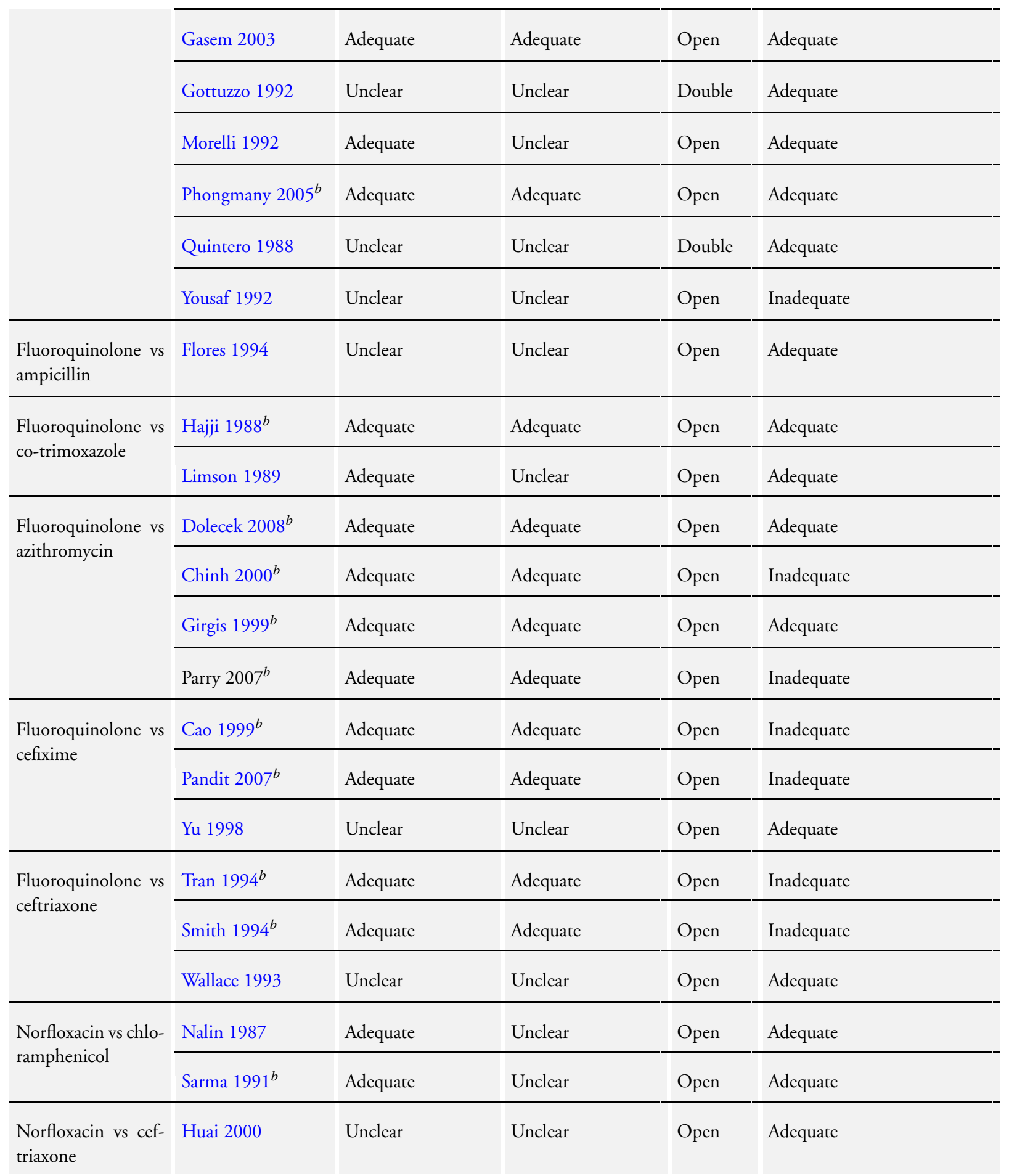


Table 5. Assessment of risk of bias ${ }^{a} \quad$ (Continued)

\begin{tabular}{|c|c|c|c|c|c|}
\hline \multirow{4}{*}{$\begin{array}{l}\text { Norfloxacin vs other } \\
\text { fluoroquinolones }\end{array}$} & Bai 1995 & Unclear & Unclear & Open & Adequate \\
\hline & Jia 1994 & Unclear & Unclear & Double & Adequate \\
\hline & Xiao 1991 & Unclear & Unclear & Open & Adequate \\
\hline & Yang 1991 & Unclear & Unclear & Open & Adequate \\
\hline \multirow{8}{*}{$\begin{array}{l}\text { Different durations } \\
\text { of fluoroquinolones }\end{array}$} & Alam 1995 & Unclear & Unclear & Open & Inadequate \\
\hline & Duong $1995^{b}$ & Adequate & Adequate & Open & Inadequate \\
\hline & Kalo 1997 & Unclear & Unclear & Open & Adequate \\
\hline & Nguyen $1997^{b}$ & Adequate & Adequate & Open & Inadequate \\
\hline & Tran $1995^{b}$ & Adequate & Adequate & Open & Inadequate \\
\hline & Unal 1996 & Unclear & Unclear & Open & Adequate \\
\hline & Vinh $1996^{b}$ & Adequate & Adequate & Open & Inadequate \\
\hline & Vinh $2005^{b}$ & Adequate & Adequate & Open & Adequate \\
\hline
\end{tabular}

a See 'Data collection and analysis' for the assessment methods, and the 'Characteristics of included studies' for the methods used in each trial.

${ }^{b}$ Trial author provided additional information.

\section{Trials with more than two comparison groups}

One trial compared a fluoroquinolone with two non-fluoroquinolone antibiotics (Yousaf 1992), and another trial compared a fluoroquinolone with a non-fluoroquinolone antibiotic (azithromycin) as well as a combination of both antibiotics (ofloxacin with azithromycin) (Parry 2007). We did not include the comparison of fluoroquinolone with combination of fluoroquinolone and nonfluoroquinolone. For Yousaf 1992 we separated the data into two meta-analyses: one comparing a fluoroquinolone with amoxicillin and the other with chloramphenicol.

When trials compared several different fluoroquinolones with a single non-fluoroquinolone antibiotic (Arnold 1993; Morelli 1992) or different fluoroquinolones against each other (Xiao 1991), we combined the groups treated with fluoroquinolones into a single fluoroquinolone group. For two trials, we only se- lected some groups; we included three groups out of the six for Morelli 1992 because they were common to other trials included in this review (Bai 1995; Jia 1994; Yang 1991), and only three of five groups for Xiao 1991 because each of the comparison groups had a sample size of less than 10 . We intend to include the groups in future updates if more trials become available.

\section{Heterogeneity}

We checked for heterogeneity by visually inspecting the forest plots and by using the chi-squared test for homogeneity (using a $10 \%$ level of statistical significance). When we detected heterogeneity among studies and still considered it appropriate to pool the data, we used the random-effects model. We were unable to explore the following potential sources of heterogeneity using subgroup analyses because of the limited number of trials in each compari- 
son: drug dose; severe and/or complicated enteric fever (as defined by trialists) versus uncomplicated enteric fever; and different time points for outcome measurements.

\section{Sensitivity analyses and publication bias}

The small number of trials in each comparison also prevented us from performing a sensitivity analysis for each of the methodological quality factors for all comparisons except for fluoroquinolones compared to chloramphenicol. We assessed the presence of publication bias using a funnel plot only for primary outcomes which had more than five studies.

\section{R E S U L T S}

\section{Description of studies}

See: Characteristics of included studies; Characteristics of excluded studies; Characteristics of studies awaiting classification; Characteristics of ongoing studies.

\section{Trial selection}

We assessed 70 trials for eligibility and included 38 (3279 participants) (see 'Characteristics of included studies') and excluded 25 (see 'Characteristics of excluded studies'). Among the remaining seven trials, which we assessed for eligibility, three were duplicate publications of the included studies (Hajji 1988; Arnold 1993; Jia 1994), two are ongoing (ISRCTN53258327; ISRCTN66534807; see 'Characteristics of ongoing studies'), and we were unable to retrieve two (Flores 1991; Soewandojo 1992; see'Characteristics of studies awaiting classification').

\section{Trial design and location}

Eleven trials were conducted in Vietnam, six trials in China, two trials in each of the Philippines, Mexico, and Italy, and one trial in each of Albania, Bahrain, Bangladesh, Egypt, Guatemala, India, Indonesia, Laos, Morocco, Nepal, Pakistan, and Turkey. We could not determine the location of one trial (Gottuzzo 1992). Two were multicenter trials conducted in different countries (Nalin 1987; Arnold 1993).

Most trials were small and therefore lacked statistical power to detect differences between the treatment regimens. The smallest trial had 26 participants and the largest had 287 participants.

\section{Participants}

Most trials included only adults (minimum age for adults reported by trialists was $\geq 14$ years), and only four trials exclusively included children (Vinh 1996; Cao 1999; Huai 2000; Vinh 2005).
Seven trials included children and adults (Xiao 1991; Yang 1991; Alam 1995; Tran 1995; Pandit 2007; Parry 2007; Dolecek 2008), although $87 \%, 79 \%$, and $73 \%$ of participants were children in three of these trials (Tran 1995; Parry 2007; Dolecek 2008) and were considered as trials on (mostly) children. Two of these, Alam 1995 and Pandit 2007, had 84\% and 65\% adults respectively, and were considered as a trial on (mostly) adults. Five trial reports did not mention the participants' age; however, four used adult dosages (Nalin 1987; Quintero 1988; Bran 1991; Jia 1994), and one used the keyword "adult" (Flores 1994).

All but three trials were conducted on inpatients; Alam 1995 was conducted on both inpatients and outpatients, and Tran 1995 was a community-based outpatient trial, while Pandit 2007 recruited outpatients presenting to the outpatient or emergency department of the study hospital.

Nineteen trials were conducted exclusively on participants with uncomplicated enteric fever or participants without major complications of enteric fever, and one included only participants with severe enteric fever (Cristiano 1995). (The terms "severe", "complicated", and "uncomplicated" were as defined by the trial authors.) The remaining trials either did not provide this information or included a combination.

Most trials used blood cultures or bone marrow cultures, or both, to confirm cases of enteric fever. Although three trials included stool culture-positive cases (Girgis 1999 (three cases); Hajji 1988 (nine cases); Smith 1994 (three cases)) and urine culture-positive cases (Hajji 1988 (one case)), we included them in the review since all three trials mainly included blood culture-positive cases. Five trials did not report the site of culture (Nalin 1987; Quintero 1988; Gottuzzo 1992; Yousaf 1992; Flores 1994), but based on information available, such as mention of follow up "blood cultures", we assumed that these trials included participants with predominantly blood culture-confirmed enteric fever.

\section{Inclusion and exclusion criteria}

Trialists tended to report outcomes only for culture-confirmed cases of enteric fever. Most trialists excluded culture-negative cases from detailed analysis, even if initially enrolled.

\section{Interventions}

\section{Fluoroquinolones versus non-fluoroquinolone antibiotics}

Twenty-three trials (1867 participants) compared fluoroquinolones with chloramphenicol (10 trials), amoxicillin or ampicillin (two trials), co-trimoxazole (two trials), azithromycin (four trials), ceftriaxone (three trials), or cefixime (three trials). Of these, two compared a newer fluoroquinolone (gatifloxacin) with azithromycin and cefixime respectively (Pandit 2007; Dolecek 2008). 


\section{Norfloxacin trials}

Three trials compared the fluoroquinolone norfloxacin with chloramphenicol (259 participants), one compared it with ceftriaxone (60 participants), and five trials (171 participants) compared it with pefloxacin, ofloxacin, and enoxacin.

\section{Different durations of fluoroquinolone}

Nine trials (889 participants) compared different fluoroquinolone treatment durations: 2 days with 3 days (three trials); 3 days with 5 days (two trials), 5 days with 7 days (one trial); 7 days with 10 days (one trial); 7 days with 14 days (one trial); and 10 days with 14 days (one trial).

\section{Length of fluoroquinolone treatment}

Most trials comparing fluoroquinolones (excluding norfloxacin) with a non-fluoroquinolone antibiotic treated the participants with the fluoroquinolone for seven ( 8 trials) or 10 days ( 6 trials) (range three to 15 days). Among trials comparing fluoroquinolones with first-line antibiotics (chloramphenicol, co-trimoxazole, and ampicillin or amoxicillin), only three trials used a short-course fluoroquinolone regimen of three days (Phongmany 2005) or seven days (Arnold 1993; Gasem 2003). Among 10 trials that compared a fluoroquinolone with azithromycin (four trials), ceftriaxone (three trials), or cefixime (three trials), nine used a short-course fluoroquinolone regimen of five days (three trials) or seven days (six trials). All other trials used a long-course (greater than seven days) fluoroquinolone regimen.

\section{Primary outcomes}

We were able to extract data on all three primary outcomes - clinical failure, microbiological failure, and relapse - from 24 of the 38 trials, on any two primary outcomes from eight trials, and on any one primary outcome from six trials.

There were considerable variations regarding the time points at which outcomes were measured, particularly microbiological failure (such as day two, the end of treatment, and some days after treatment) and relapse (such as during therapy and up to two months of follow up). The precise descriptions also varied considerably; for example, some trialists defined "relapse" as the recurrence of similar signs and symptoms with confirmation by blood and/or bone marrow culture (sterile site, as defined in protocol), and others as confirmed by positive stool cultures (non-sterile site) only. Some trialists did not explicitly state how they confirmed relapse in their trial (see Table 3 'Definitions of outcomes').

\section{Secondary outcomes}

Twenty-eight trials reported mean fever clearance times, but six did not report the standard deviation or the information required to calculate a standard deviation. The $95 \%$ confidence intervals for the mean were reported in three trials (Chinh 2000; Vinh 2005; Parry 2007) from which we derived the standard deviation. Fever clearance times are usually skewed: several trials used non-parametric tests of statistical significance, and authors provided mean and standard deviation on request for four trials that reported a median fever clearance time in the original report (Cao 1999; Phongmany 2005; Pandit 2007; Dolecek 2008). The Student's $t$ test was also used (it is unclear whether data were log transformed before conducting this test). Caution is required when interpreting the mean difference for fever clearance times, as there may be some skew, and the denominator in calculating mean fever clearance times was not clear in most trials. Since we could not ascertain whether trial authors had excluded "clinical failures" in their calculation of mean fever clearance times (or included the fever clearance time of participants who were switched over to another drug), the mean fever clearance times included in our meta-analyses could be biased.

We could extract data on the length of hospitalization from 12 trials, complications from 20 trials, serious adverse events from 28 trials, and other adverse events from 23 trials. Five trials reported laboratory adverse events distinct from clinical adverse events ( Nalin 1987; Hajji 1988; Yang 1991; Abejar 1993; Smith 1994). We extracted these laboratory data but did not analyse them together with clinical adverse events to avoid the overlap of participants. Four trials reported the number of adverse events, and not the number of participants (Morelli 1992; Duong 1995; Girgis 1999; Chinh 2000). Only one author provided the cost of therapy as additional data (Girgis 1999).

Twenty-five trials measured convalescent faecal carriage, but we could not extract data from three trials because one included three participants who were not blood culture confirmed at enrolment ( Arnold 1993), and two because the participants with positive stool cultures at follow up also had recurrence of symptoms and were reported as relapses (Wallace 1993; Unal 1996).

\section{MDR and $\mathrm{NaR}$ strains}

See Table 1.

Of the 38 included trials, only 13 trials reported the proportion of participants with $\mathrm{NaR}$ strains, among which it was absent in four trials (Hajji 1988; Smith 1994; Cao 1999; Phongmany 2005). The MICs of different fluoroquinolones reported in trials that did not report $\mathrm{NaR}$ strains ranged from $<0.016$ to $8 \mathrm{mg} / \mathrm{L}$, suggesting that some strains may have had reduced susceptibility to fluoroquinolones (reference ranges available in (CLSI 2006)), but precise numbers of such strains in each arm were not available.

One of the two trials comparing norfloxacin with chloramphenicol did not report the proportion of participants with MDR strains. Among 13 trials comparing other fluoroquinolones (excluding norfloxacin) with first-line antibiotics, MDR strains were present in one trial (Phongmany 2005) absent in eight trials, and four 
trials did not report on this (Gottuzzo 1992; Yousaf 1992; Arnold 1993; Flores 1994).

\section{Risk of bias in included studies}

See risk of bias summary in Table 5 .

\section{Generation of allocation sequence}

Twenty-one trials used an adequate randomization method: eight used random-numbers lists or tables; and the rest were computer generated. Five of these trials used block randomization. The method used to generate the allocation sequence in the remaining 17 trials was unclear.

\section{Allocation concealment}

Sixteen trials used an adequate method (sealed envelopes) to conceal allocation. The method used in the remaining 22 trials was unclear.

\section{Blinding}

Four trials were described as "double blinded" and 34 trials were open; two trials did not mention use of placebo, but we assumed they were open (Flores 1994; Bai 1995).

\section{Inclusion of all randomized culture-positive participants in the final analysis}

We obtained the percentage of participants lost to follow up by dividing the number of culture-positive participants unaccounted for at follow up by the total number of culture-positive participants randomized. Thus 26 trials included an adequate ( $90 \%$ or more) number of participants in final analysis (or assumed to be adequate when there was no mention of losses to follow up), and 12 trials included an inadequate number (less than 90\%).

\section{Overall methodological quality}

Only six open trials used adequate methods to generate the allocation sequence and conceal allocation, and had few or no losses at the final follow up for which data were reported (Hajji 1988; Girgis 1999; Gasem 2003; Phongmany 2005; Vinh 2005; Dolecek 2008). Ten other open trials used adequate methods of randomization and allocation concealment but did not have adequate follow up (Smith 1994; Tran 1994; Duong 1995; Tran 1995; Vinh
1996; Nguyen 1997; Cao 1999; Chinh 2000; Pandit 2007; Parry 2007).

Five open trials reported an adequate method of randomization and follow up (Nalin 1987; Limson 1989; Sarma 1991; Morelli 1992; Cristiano 1995). Four trials were double-blinded trials with adequate follow up (Quintero 1988; Bran 1991; Gottuzzo 1992; Jia 1994). Two open trials did not have any adequate quality measure (Yousaf 1992; Alam 1995), and the remaining 11 trials had only adequate follow up.

\section{Effects of interventions}

\section{Fluoroquinolones versus first-line antibiotics (chloramphenicol, co-trimoxazole, and ampicillin or amoxicillin)}

\section{I.I. Fluoroquinolones versus chloramphenicol}

Ten trials made this comparison (Quintero 1988; Bran 1991; Gottuzzo 1992; Morelli 1992; Yousaf 1992; Abejar 1993; Arnold 1993; Cristiano 1995; Gasem 2003; Phongmany 2005). Three did not clarify the proportion of participants with MDR strains (Gottuzzo 1992; Yousaf 1992; Arnold 1993), and nine did not report $\mathrm{NaR}$ data, while this was absent in one trial (Phongmany 2005). One trial included five different fluoroquinolones ofloxacin, pefloxacin, ciprofloxacin, enoxacin, and norfloxacin ( Morelli 1992); we analysed the norfloxacin group separately, as explained above.

\section{Clinical failure}

We did not detect any statistically significant difference in clinical failure (Analysis 1.1, Figure 1) in trials that included participants without MDR strains (307 participants, 5 trials), participants with some MDR strains (50 participants, 1 trial), or an unknown proportion of participants with MDR strains (237 participants, 3 trials), or when we combined all subgroups (594 participants, 9 trials). In one included trial (Phongmany 2005), participants randomized to chloramphenicol when found infected with a chloramphenicol-resistant isolate were switched to the fluoroquinolone group and consequently did not experience clinical failure. These were restored to their originally randomized groups by the authors (with no clinical failures reported in chloramphenicol arm) (Phongmany 2005). 
Figure I. Fluoroquinolones vs chloramphenicol: clinical failure

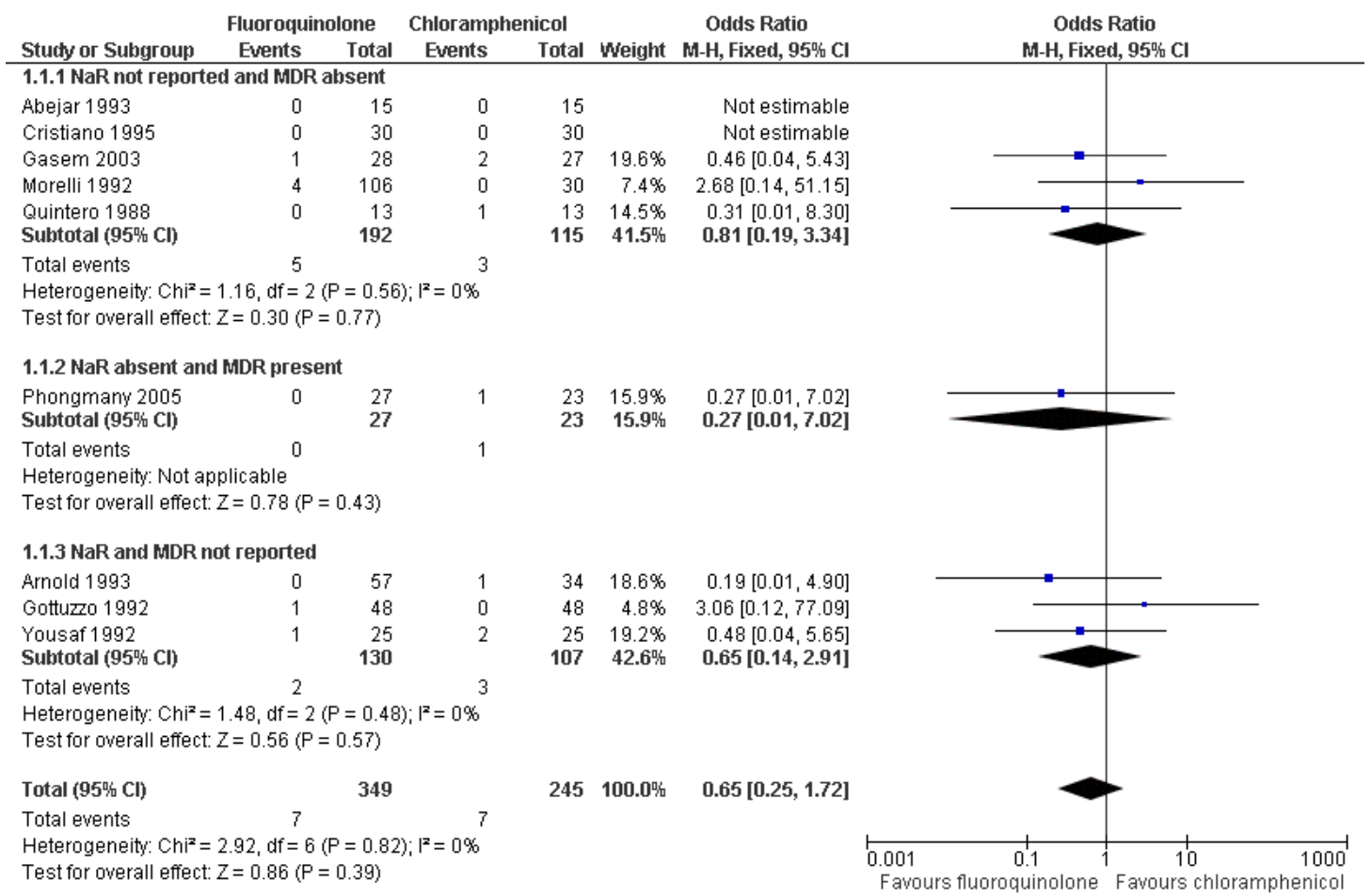

We did not detect any statistically significant difference in clinical failure when we included only trials with adequate methodological quality (adequate methods of randomization and adequate allocation concealment, and no losses to follow up) (105 participants; Gasem 2003; Phongmany 2005; Analysis not shown).

\section{Microbiological failure}

We did not detect any statistically significant difference in the odds of microbiological failure in people without MDR strains (237 participants, 4 trials, Analysis 1.2). (Gasem 2003 performed both blood and bone marrow cultures for half of the participants at day three and for the other half of participants at day five of treatment to assess microbiological failure. We combined the blood culture results for both days, but we did not use bone marrow culture results as they were conducted on the same participants whose blood cultures were also taken.) In trials with an unknown proportion of participants with MDR strains (Analysis 1.2), the odds of microbiological failure were statistically significantly lower in the fluoroquinolone group (OR 0.24, 95\% CI 0.06 to 0.96 ; 141 participants, 2 trials), but the results that favoured fluoroquinolones were of borderline statistical significance when we combined the two subgroups (OR $0.43,95 \%$ CI 0.18 to 1.03 ; 378 participants,

6 trials).

We did not detect any statistically significant difference in microbiological failure when we included only trials with adequate methodological quality (adequate methods of randomization and adequate allocation concealment, and no losses to follow up) (45 participants, Gasem 2003, Analysis 1.2).

\section{Relapse}

In trials with no participants with MDR strains, the odds of relapse were reduced by $88 \%$, which is statistically significant (OR $0.12,95 \%$ CI 0.02 to $0.69 ; 281$ participants, 4 trials, Analysis 1.3 , Figure 2). However, we could not determine the precise definition or the site of culture to confirm a relapse in most of the trials (see Table 3), including Morelli 1992, which received the greatest weight in this meta-analysis. In the two trials with an unknown proportion of participants with MDR strains, we did not detect any statistically significant differences, but statistical power was very limited (186 participants, 2 trials, Analysis 1.3). The odds of relapse were reduced significantly when we combined both subgroups (OR 0.14, 95\% CI 0.04 to 0.50; 467 participants, 6 trials, Analysis 1.3). When we excluded from the analysis those trials that did not clearly define relapse (Abejar 1993; Cristiano 1995), 
did not confirm relapse using cultures (Gasem 2003), or did not define relapse at all (Gottuzzo 1992; Morelli 1992), thus retaining only one trial (91 participants) with clearly blood culture-confirmed relapses (Arnold 1993) (sterile site-culture confirmed, as defined in the protocol), we did not find any difference between fluoroquinolones and chloramphenicol.

Figure 2. Fluoroquinolones vs chloramphenicol: relapse

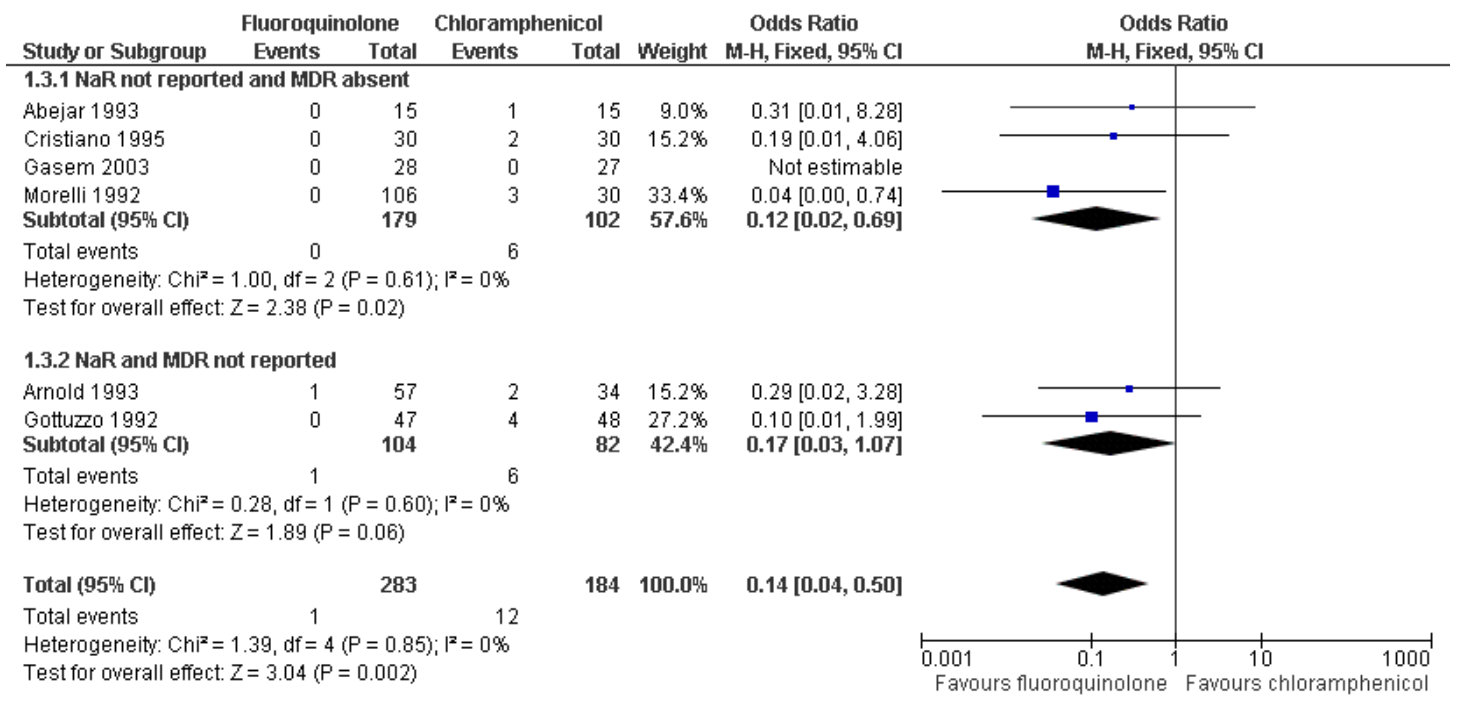

The only trial with adequate methodological quality in this comparison did not report any relapses (55 participants, Gasem 2003, Analysis 1.3); however this was only reported during hospitalization, and longer follow up was not done.

\section{Fever clearance time}

The meta-analysis of two trials without participants with MDR strains tended to favour fluoroquinolones for fever clearance time, but the sample size was small so that the confidence intervals were very wide (MD -16.07 hours, $95 \%$ CI -35.03 to 2.88 ; 81 participants, Analysis 1.4). One trial (Phongmany 2005) with NaR absent and MDR present reported a statistically significantly lower mean fever clearance time in the fluoroquinolone group (MD 38.5 hours, $95 \%$ CI -59.90 to -17.10 ; 48 participants, Analysis 1.4). On combining both subgroups we detected a statistically significantly lower mean fever clearance time in the fluoroquinolone group (MD -25.93 hours, 95\% CI -40.12 to $-11.74 ; 129$ participants, 3 trials, Analysis 1.4). Fever clearance time was slightly shorter in both Abejar 1993 and Cristiano 1995, but the trialists did not report standard deviations or precise results of tests of sta- tistical significance, and we could not include them in the metaanalysis.

When we included only two above-mentioned trials with adequate methodological quality (Gasem 2003; Phongmany 2005), we detected a statistically significantly lower mean fever clearance time in the fluoroquinolone group (MD -27.56 hours, 95\% CI -43.38 to -11.75 ; 103 participants, Analysis not shown).

\section{Length of hospital stay}

Three trials reported on the length of hospital stay (Analysis 1.5). One of them, Cristiano 1995, did not report the standard deviation (fluoroquinolone group: mean 5 days, range 3 to 8 days; chloramphenicol group: mean 5.53 days, range 2 to 8 days). We did not detect any statistically significant difference in the length of hospital stay in the other trial, Gasem 2003 (55 participants), which had no MDR and did not report NaR. One trial reported a statistically significantly lower mean length of hospital stay in the fluoroquinolone group (MD -5.90 days, 95\% CI -7.42 to 4.38; 50 participants, Phongmany 2005). On combining both 
subgroups we detected a statistically significantly lower length of hospital stay in the fluoroquinolone group (MD -2.57 days, 95\% CI -3.53 to -1.62 ; 105 participants, 2 trials). We detected statistically significant heterogeneity in the two trials; this could be due to differences in durations of fluoroquinolone treatment (15 days versus 3 days) in the two trials. We did not detect any statistically significant difference in the length of hospital stay when we used a random-effects model to combine the trials (105 participants, 2 trials, Analysis not shown). These were the only two trials that reported length of hospital stay, and both were of adequate methodological quality.

\section{Convalescent faecal carriage}

We found a statistically significant decrease in the odds of convalescent faecal carriage in the fluoroquinolone group in trials that did not have any participants with MDR strains (OR 0.17, 95\% CI 0.04 to $0.70 ; 298$ participants, 3 trials, Analysis 1.6). No trial of adequate methodological quality reported this outcome.

\section{Complications and adverse events}

We did not detect a statistically significant difference in the number of complications in two trials, both of adequate methodological quality, that reported these data (105 participants, Analysis 1.7). The complications included pneumonia, sepsis, myocarditis, gastrointestinal bleeding, and perforation (see Table 2). We did not detect a statistically significant difference in the number of nonserious adverse events, such as nausea, epigastric pain, skin rash, headache, and dizziness (245 participants, 5 trials, Analysis 1.8; see Table 6). We also did not detect any statistically significant differences in non-serious adverse events in the two trials with adequate methodological quality (105 participants, Analysis not shown; see Table 6). One trial mentioned selected serious adverse events, including one participant with severe rash in the ciprofloxacin group and one with severe leucopenia in the chloramphenicol group ( Gottuzzo 1992; Table 7).

Table 6. Non-serious adverse events ${ }^{a}$

\begin{tabular}{|c|c|c|c|c|c|}
\hline \multirow[t]{2}{*}{ Comparison } & \multirow[t]{2}{*}{ Trial } & \multicolumn{2}{|c|}{$\begin{array}{l}\text { No. of participants (in brackets except } \\
\text { where specifically stated) with non-seri- } \\
\text { ous adverse events }{ }^{b}\end{array}$} & \multicolumn{2}{|c|}{ Laboratory adverse events ${ }^{c}$} \\
\hline & & Intervention & Control & Intervention & Control \\
\hline $\begin{array}{l}\text { Fluoroquinolone vs } \\
\text { chloramphenicol }\end{array}$ & Abejar 1993 & $\begin{array}{l}\text { Fleroxacin: } \\
\text { numbness in upper } \\
\text { extremities (2) }\end{array}$ & $\begin{array}{l}\text { Chloram- } \\
\text { phenicol: numbness } \\
\text { in upper extremities } \\
\text { (1) }\end{array}$ & $\begin{array}{l}\text { Flerox- } \\
\text { acin: increased crea- } \\
\text { tinine }(4)\end{array}$ & $\begin{array}{l}\text { Chloramphenicol: } \\
\text { increased creatinine } \\
\text { (4); increased blood } \\
\text { urea nitrogen (2) }\end{array}$ \\
\hline
\end{tabular}

Fluoroquinolones for treating typhoid and paratyphoid fever (enteric fever) (Review)

Copyright ( 2008 The Cochrane Collaboration. Published by John Wiley \& Sons, Ltd. 
Table 6. Non-serious adverse events ${ }^{a} \quad$ (Continued)

\begin{tabular}{|c|c|c|c|c|}
\hline Arnold 1993 & $\begin{array}{l}\text { Seborrheic dermati- } \\
\text { tis in 14-day fluoro- } \\
\text { quinolone group (1) } \\
\text { Gastrointesti- } \\
\text { nal complaints were } \\
\text { most common, in- } \\
\text { clud- } \\
\text { ing nausea and vom- } \\
\text { iting; insomnia also } \\
\text { reported; no. were } \\
\text { not provided for cul- } \\
\text { ture-positive partic- } \\
\text { ipants but reported } \\
\text { together with cul- } \\
\text { ture-negative partic- } \\
\text { ipants }\end{array}$ & $\begin{array}{l}\text { Gastrointesti- } \\
\text { nal complaints were } \\
\text { most common, in- } \\
\text { clud- } \\
\text { ing nausea and vom- } \\
\text { iting; insomnia also } \\
\text { reported; no. were } \\
\text { not provided for cul- } \\
\text { ture-positive partic- } \\
\text { ipants but reported } \\
\text { together with cul- } \\
\text { ture-negative partic- } \\
\text { ipants }\end{array}$ & \multicolumn{2}{|c|}{$\begin{array}{l}\text { Most frequent laboratory abnormalities in- } \\
\text { cluded low neutrophil or total leukocyte } \\
\text { count, low haemoglobin and haematocrit, } \\
\text { but it does not say which event occurred in } \\
\text { which arm and are reported together with } \\
\text { culture-negative participants }\end{array}$} \\
\hline Bran 1991 & 0 & 0 & 0 & 0 \\
\hline Cristiano 1995 & $\begin{array}{l}\text { Pe- } \\
\text { floxacin: nausea (3); } \\
\text { epigastric pain (3); } \\
\text { transient rash (1) }\end{array}$ & $\begin{array}{l}\text { Chloramphenicol: } \\
\text { epigastric pain }(5)\end{array}$ & 0 & 0 \\
\hline Gasem 2003 & Ciprofloxacin: 0 & $\begin{array}{l}\text { Chloramphenicol: } \\
\text { rash (1) }\end{array}$ & Ciprofloxacin: 0 & $\begin{array}{l}\text { Chlorampheni- } \\
\text { col: mean decrease } \\
\text { in haemoglobin lev- } \\
\text { els (no. not stated) }\end{array}$ \\
\hline Gottuzzo 1992 & $\begin{array}{l}\text { Ciprofloxacin: not } \\
\text { described }\end{array}$ & $\begin{array}{l}\text { Chlorampheni- } \\
\text { col: leucopenia (10); } \\
\text { others not described }\end{array}$ & Not described & Not described \\
\hline Morelli 1992 & $\begin{array}{l}\text { Events in } 4 \text { fluo- } \\
\text { roquinolone groups: } \\
\text { epigastric pain (26); } \\
\text { flushing (12); } \\
\text { headache (8); dizzi- } \\
\text { ness (11); skin rash } \\
(4)\end{array}$ & $\begin{array}{l}\text { Events in chloram- } \\
\text { phenicol group: di- } \\
\text { arrhoea (3); epigas- } \\
\text { tric pain (6); abdom- } \\
\text { inal pain (4) }\end{array}$ & Not described & Not described \\
\hline Phongmany 2005 & 0 & 0 & - & - \\
\hline Yousaf 1992 & Ofloxacin: (3) & $\begin{array}{l}\text { Chloramphenicol: } \\
\text { (4) }\end{array}$ & Not described & Not described \\
\hline
\end{tabular}


Table 6. Non-serious adverse events ${ }^{a} \quad$ (Continued)

\begin{tabular}{|c|c|c|c|c|c|}
\hline $\begin{array}{l}\text { Fluoroquinolone vs } \\
\text { ampicillin or amox- } \\
\text { icillin }\end{array}$ & Yousaf 1992 & Ofloxacin: (3) & $\begin{array}{l}\text { Amoxicillin: (11) } \\
\text { (mostly diarrhoea, } \\
\text { pruritis, rash) }\end{array}$ & Not described & Not described \\
\hline \multirow[t]{2}{*}{$\begin{array}{l}\text { Fluoroquinolone vs } \\
\text { co-trimoxazole }\end{array}$} & Најji 1988 & $\begin{array}{l}\text { Pefloxacin: photoxi- } \\
\text { city (1) }\end{array}$ & $\begin{array}{l}\text { Co-trimoxazole: } \\
\text { rash (1) }\end{array}$ & $\begin{array}{l}\text { Pefloxacin: increased } \\
\text { transaminase }(2)\end{array}$ & $\begin{array}{l}\text { Co-tri- } \\
\text { moxazole: increased } \\
\text { transaminases }(1)\end{array}$ \\
\hline & Limson 1989 & $\begin{array}{l}\mathrm{Ci}- \\
\text { profloxacin: abdom- } \\
\text { inal discomfort (4); } \\
\text { dizziness (1) }\end{array}$ & $\begin{array}{l}\text { Co-trimoxa- } \\
\text { zole: nausea (5); pru- } \\
\text { ritis (1) }\end{array}$ & 0 & 0 \\
\hline \multirow[t]{4}{*}{$\begin{array}{l}\text { Fluoroquinolone vs } \\
\text { azithromycin }\end{array}$} & Dolecek 2008 & $\begin{array}{l}\text { Gatifloxacin: vomit- } \\
\text { ing (1); diarrhoea (1) }\end{array}$ & $\begin{array}{l}\text { Azithromycin: rash } \\
\text { (1) }\end{array}$ & - & - \\
\hline & Chinh 2000 & $\begin{array}{l}\text { No. } \\
\text { events for ofloxacin: } \\
\text { nausea (1); vomiting } \\
\text { (3); abdominal pain } \\
\text { (4) }\end{array}$ & $\begin{array}{l}\text { No. events for } \\
\text { azithromycin: nau- } \\
\text { sea (5); vomiting (5); } \\
\text { abdominal pain (4); } \\
\text { rash (1) }\end{array}$ & $\begin{array}{l}\text { Ofloxacin: increased } \\
\text { mean lev- } \\
\text { els of alanine and as- } \\
\text { partate aminotrans- } \\
\text { ferase (all } 44 \text { partici- } \\
\text { pants) }\end{array}$ & $\begin{array}{l}\text { Azithromycin: } \\
\text { increased mean lev- } \\
\text { els of alanine and as- } \\
\text { partate aminotrans- } \\
\text { ferase (all } 44 \text { partici- } \\
\text { pants) }\end{array}$ \\
\hline & Girgis 1999 & $\begin{array}{l}\text { Ciprofloxacin: nau- } \\
\text { sea and vomiting } \\
\text { (4); lightheadedness } \\
\text { (2); dry mouth (4); } \\
\text { loose stools (3); con- } \\
\text { stipation (2) }\end{array}$ & $\begin{array}{l}\text { Azithromycin: nau- } \\
\text { sea and vomiting } \\
\text { (6); lightheadedness } \\
\text { (2); dry mouth (3); } \\
\text { loose stools (3); con- } \\
\text { stipation (2) }\end{array}$ & $\begin{array}{l}\text { Ciproflo- } \\
\text { xacin: thrombocyto- } \\
\text { sis (1); mild aspar- } \\
\text { tate transaminase in- } \\
\text { crease (3) }\end{array}$ & $\begin{array}{l}\text { Azithro- } \\
\text { mycin: thrombocy- } \\
\text { tosis }(4) \text {; mild aspar- } \\
\text { tate transaminase in- } \\
\text { crease (2) }\end{array}$ \\
\hline & Parry 2007 & $\begin{array}{l}\text { Ofloxacin: joint dis- } \\
\text { comfort which re- } \\
\text { solved (1); gastroin- } \\
\text { testinal side effects } \\
\text { (no. not stated) }\end{array}$ & $\begin{array}{l}\text { Azithro- } \\
\text { mycin: joint discom- } \\
\text { fort which resolved } \\
\text { (1); gastrointestinal } \\
\text { side effects (no. not } \\
\text { stated) }\end{array}$ & - & - \\
\hline \multirow[t]{2}{*}{$\begin{array}{l}\text { Fluoroquinolone vs } \\
\text { cefixime }\end{array}$} & Cao 1999 & $\begin{array}{l}\text { No. } \\
\text { events for ofloxacin: } \\
\text { abdominal pain (4); } \\
\text { diarrhoea (4); vom- } \\
\text { iting (1) }\end{array}$ & $\begin{array}{l}\text { No. events for ce- } \\
\text { fixime group: ab- } \\
\text { dominal pain (1); di- } \\
\text { arrhoea (4); vomit- } \\
\text { ing (1); rash (1) }\end{array}$ & Not described & Not described \\
\hline & Pandit 2007 & $\begin{array}{l}\text { Gatifloxacin: nausea } \\
\text { and vomiting (18). } \\
\text { Among these, } 2 \text { with } \\
\text { ex- } \\
\text { cessive vomiting re- }\end{array}$ & $\begin{array}{l}\text { Cefixime: nausea } \\
\text { and vomiting }(1)\end{array}$ & - & - \\
\hline
\end{tabular}

Fluoroquinolones for treating typhoid and paratyphoid fever (enteric fever) (Review)

Copyright @ 2008 The Cochrane Collaboration. Published by John Wiley \& Sons, Ltd. 
Table 6. Non-serious adverse events ${ }^{a} \quad$ (Continued)

\begin{tabular}{|l|l|} 
quired intravenous \\
antiemetics and flu- \\
ids and observation \\
in the hospital emer- \\
gency room for up \\
to 6 hours; 2 needed \\
oral antiemetics
\end{tabular}

Yu 1998 Levofloxacin: 0 Cefixime:

Yu 1998 Levofloxacin: 0 Cefixime: nausea Lev- Cefixime: increased and low appetite (2) ofloxacin: increased alanine aminotransalanine aminotrans- ferase (1) ferase (2)

\begin{tabular}{|c|c|c|c|c|c|}
\hline $\begin{array}{l}\text { Fluoroquinolone vs } \\
\text { ceftriaxone }\end{array}$ & Smith 1994 & $\begin{array}{l}\text { Ofloxacin: pruritis } \\
\text { (1) }\end{array}$ & $\begin{array}{l}\text { Ceftriaxone: skin } \\
\text { rash }(2)\end{array}$ & $\begin{array}{l}\text { Ofloxacin: } \\
\text { mildly increased cre- } \\
\text { atinine (1) }\end{array}$ & Ceftriaxone: 0 \\
\hline \multirow[t]{2}{*}{$\begin{array}{l}\text { Norfloxacin vs chlo- } \\
\text { ramphenicol }\end{array}$} & Nalin 1987 & $\begin{array}{l}\text { Norfloxacin: clinical } \\
\text { adverse events }(6)\end{array}$ & $\begin{array}{l}\text { Chloram- } \\
\text { phenicol: clinical ad- } \\
\text { verse events ( } 7 \text { ) }\end{array}$ & $\begin{array}{l}\text { Norfloxacin: labora- } \\
\text { tory adverse events } \\
\text { (10) }\end{array}$ & $\begin{array}{l}\text { Chloram- } \\
\text { phenicol: laboratory } \\
\text { adverse events }(20)\end{array}$ \\
\hline & Sarma 1991 & $\begin{array}{l}\text { Norfloxacin: nausea } \\
\text { (3); vomiting (3); } \\
\text { headache (2) }\end{array}$ & Chloramphenicol: 0 & Norfloxacin: 0 & $\begin{array}{l}\text { Chlorampheni- } \\
\text { col: decreased mean } \\
\text { hematocrit ( } 2) \text {, de- } \\
\text { creased white blood } \\
\text { cell } \\
\text { count (3), decreased } \\
\text { platelet count (2) }\end{array}$ \\
\hline $\begin{array}{l}\text { Norfloxacin vs cef- } \\
\text { triaxone }\end{array}$ & Huai 2000 & $\begin{array}{l}\text { Norfloxacin: nausea } \\
\text { and vomiting ( } 3 \text { ) }\end{array}$ & $\begin{array}{l}\text { Cef- } \\
\text { triaxone: abdominal } \\
\text { discomfort and nau- } \\
\text { sea (1) }\end{array}$ & Not described & Not described \\
\hline \multirow[t]{3}{*}{$\begin{array}{l}\text { Norfloxacin vs other } \\
\text { fluoroquinolones }\end{array}$} & Bai 1995 & Enoxacin: rash (1) & $\begin{array}{l}\text { Norfloxacin: ab- } \\
\text { dominal discomfort } \\
\text { (1) }\end{array}$ & Not described & Not described \\
\hline & Jia 1994 & Norfloxacin: 0 & $\begin{array}{l}\text { Pefloxacin: included } \\
\text { nausea, } \\
\text { vomiting, dizziness, } \\
\text { measles like rash and } \\
\text { abdominal discom- } \\
\text { fort (no. not stated) }\end{array}$ & Not described & Not described \\
\hline & Morelli 1992 & $\begin{array}{l}\text { Norfloxacin: epigas- } \\
\text { tric pain (6); flush- } \\
\text { ing (4); dizziness ( } 4 \text { ) }\end{array}$ & $\begin{array}{l}\text { No. events } \\
\text { for ofloxacin: epigas- } \\
\text { tric pain (4); flush- } \\
\text { ing (4); headache (2) }\end{array}$ & Not described & Not described \\
\hline
\end{tabular}


Table 6. Non-serious adverse events ${ }^{a} \quad$ (Continued)

\begin{tabular}{|c|c|c|c|c|c|}
\hline & & & $\begin{array}{l}\text { No. events for pe- } \\
\text { floxacin: rash (2); } \\
\text { headache (6); epi- } \\
\text { gastric pain (10) } \\
\text { No. events } \\
\text { for enoxacin: epigas- } \\
\text { tric pain (3); flush } \\
\text { (4); dizziness (7) }\end{array}$ & & \\
\hline & Xiao 1991 & $\begin{array}{l}\text { Norfloxacin: not de- } \\
\text { scribed }\end{array}$ & $\begin{array}{l}\text { Pefloxacin: nau- } \\
\text { sea and anorexia (2); } \\
\text { agitation and abnor- } \\
\text { mal behaviour (1) } \\
\text { Ofloxacin: nausea } \\
\text { (2); measles like rash } \\
\text { (3); salivation (1) }\end{array}$ & Not described & Not described \\
\hline & Yang 1991 & Ofloxacin: rash (1) & $\begin{array}{l}\text { Norfloxacin: rash } \\
\text { (1) }\end{array}$ & $\begin{array}{l}\text { Norfloxacin: } \\
\text { increased alanine } \\
\text { aminotransferase (1) }\end{array}$ & - \\
\hline \multirow[t]{4}{*}{$\begin{array}{l}\text { Different durations } \\
\text { of fluoroquinolones }\end{array}$} & Alam 1995 & $\begin{array}{l}\text { 10-day: } \\
4 \text { participants had } \\
11 \text { events including } \\
\text { malaise (1), dizziness } \\
(1) \text {, nausea (1), in- } \\
\text { somnia (1), rash (1), } \\
\text { pruritis (1), lethargy } \\
(1) \text {, weakness (1), } \\
\text { and headache (3) }\end{array}$ & $\begin{array}{l}\text { 14-day: } 9 \text { partici- } \\
\text { pants had } 18 \text { events } \\
\text { including joint pain } \\
\text { (1), malaise (3), ab- } \\
\text { dominal pain (3), } \\
\text { headache (1), dizzi- } \\
\text { ness (1), nausea (1), } \\
\text { oral mucosal pain } \\
\text { (2), insomnia (1), } \\
\text { vomiting (1), vertigo } \\
\text { (1), palpitations (1), } \\
\text { and photosensitivity } \\
\text { (1) }\end{array}$ & $\begin{array}{l}\text { 10-day: moderate } \\
\text { eosinophilia }(5)\end{array}$ & $\begin{array}{l}\text { 14-day: moder- } \\
\text { ate eosinophilia ( } 3 \text { ); } \\
\text { increased serum cre- } \\
\text { atinine (1) }\end{array}$ \\
\hline & Duong 1995 & $\begin{array}{l}\text { 3-day: insomnia } \\
\text { (10) }\end{array}$ & $\begin{array}{l}\text { No. events for 5-day: } \\
\text { insomnia (17), nau- } \\
\text { sea and vomiting (1) }\end{array}$ & Not described & Not described \\
\hline & Kalo 1997 & $\begin{array}{l}\text { 7-day: nausea, vom- } \\
\text { iting, or abdominal } \\
\text { discomfort ( } 4 \text { ), but } \\
\text { it does not say in } \\
\text { which group }\end{array}$ & $\begin{array}{l}\text { 10-day: nau- } \\
\text { sea, vomiting, or ab- } \\
\text { dominal discomfort } \\
\text { (4), but it does not } \\
\text { say in which group }\end{array}$ & Not described & Not described \\
\hline & Tran 1995 & $\begin{array}{l}\text { 3-day (11): partici- } \\
\text { pants had insomnia, } \\
\text { dizziness, epigastric }\end{array}$ & $\begin{array}{l}\text { 5-day } \\
\text { (4): participants had } \\
\text { insomnia, dizziness, }\end{array}$ & Not described & Not described \\
\hline
\end{tabular}


Table 6. Non-serious adverse events ${ }^{a} \quad$ (Continued)

\begin{tabular}{|c|c|c|c|c|}
\hline & $\begin{array}{l}\text { pain, nausea, diar- } \\
\text { rhoea, headache and } \\
>1 \text { symptom; joint } \\
\text { symptoms reported } \\
\text { were not thought to } \\
\text { be fluoroquinolone- } \\
\text { induced } \\
\text { Details were } \\
\text { reported for culture- } \\
\text { positive participants } \\
\text { together with cul- } \\
\text { ture-negative partic- } \\
\text { ipants }\end{array}$ & $\begin{array}{l}\text { vomiting, rash; joint } \\
\text { symptoms reported } \\
\text { were not thought to } \\
\text { be fluoroquinolone- } \\
\text { induced } \\
\text { Details were } \\
\text { reported for culture- } \\
\text { positive participants } \\
\text { together with- } \\
\text { culture negative par- } \\
\text { ticipants }\end{array}$ & & \\
\hline Unal 1996 & $\begin{array}{l}\text { 5-day: nausea and } \\
\text { vomiting (3) }\end{array}$ & $\begin{array}{l}\text { 7-day: nausea and } \\
\text { vomiting ( } 3 \text { ) }\end{array}$ & 5-day: 0 & $\begin{array}{l}\text { 7-day: increase in } \\
\text { transaminases (1) }\end{array}$ \\
\hline Vinh 1996 & 2-day: 0 & 3-day: urticaria (1) & Not described & Not described \\
\hline Vinh 2005 & 2-day: 0 & 3-day: 0 & - & - \\
\hline
\end{tabular}

${ }^{a}$ Only trials reporting on non-serious adverse events are included.

${ }^{b}$ Zero (0) events only when specifically stated by trial author.

${ }^{c}$ Whenever this was reported separately.

Table 7. Serious adverse events ${ }^{a}$

\begin{tabular}{|c|c|c|c|}
\hline \multirow[t]{2}{*}{ Comparison } & \multirow[t]{2}{*}{ Trial $^{*}$} & \multicolumn{2}{|c|}{ No. participants (in brackets) with serious adverse events ${ }^{b}$} \\
\hline & & Intervention & Control \\
\hline \multirow[t]{7}{*}{$\begin{array}{l}\text { Fluoroquinolone vs chloram- } \\
\text { phenicol }\end{array}$} & Arnold 1993 & \multicolumn{2}{|c|}{$\begin{array}{l}\text { Urinary retention (1), but it does not say in which group or if a culture-negative } \\
\text { or culture-positive participant }\end{array}$} \\
\hline & Bran 1991 & 0 & 0 \\
\hline & Cristiano 1995 & 0 & 0 \\
\hline & Gasem 2003 & 0 & 0 \\
\hline & Gottuzzo 1992 & $\begin{array}{l}\text { Ciprofloxacin: severe rash (1); others } \\
\text { not described }\end{array}$ & $\begin{array}{l}\text { Chloramphenicol: severe leucopenia } \\
\text { (1); others not described }\end{array}$ \\
\hline & Phongmany 2005 & 0 & 0 \\
\hline & Quintero 1988 & 0 & 0 \\
\hline
\end{tabular}


Table 7. Serious adverse events ${ }^{a}$ (Continued)

\begin{tabular}{|c|c|c|c|}
\hline Fluoroquinolone vs ampicillin & Flores 1994 & 0 & 0 \\
\hline \multirow{2}{*}{$\begin{array}{l}\text { Fluoroquinolone vs co-trimox- } \\
\text { azole }\end{array}$} & Hajji 1988 & 0 & 0 \\
\hline & Limson 1989 & 0 & 0 \\
\hline \multirow{4}{*}{$\begin{array}{l}\text { Fluoroquinolone vs azithromy- } \\
\text { cin }\end{array}$} & Dolecek 2008 & 0 & 0 \\
\hline & Chinh 2000 & 0 & 0 \\
\hline & Girgis 1999 & 0 & 0 \\
\hline & Parry 2007 & 0 & 0 \\
\hline Fluoroquinolone vs cefixime & Pandit 2007 & 0 & 0 \\
\hline \multirow[t]{2}{*}{ Fluoroquinolone vs ceftriaxone } & Smith 1994 & 0 & 0 \\
\hline & Tran 1994 & Fleroxacin: 0 & Ceftriaxone: anaphylaxis (1) \\
\hline \multirow[t]{2}{*}{$\begin{array}{l}\text { Norfloxacin vs chlorampheni- } \\
\text { col }\end{array}$} & Nalin 1987 & $\begin{array}{l}\text { Nausea and vomiting "considered seri- } \\
\text { ous by the investigator": number with } \\
\text { event unclear }\end{array}$ & 0 \\
\hline & Sarma 1991 & 0 & 0 \\
\hline \multirow{4}{*}{$\begin{array}{l}\text { Norfloxacin vs other fluoro- } \\
\text { quinolones }\end{array}$} & Bai 1995 & 0 & 0 \\
\hline & Jia 1994 & 0 & 0 \\
\hline & Xiao 1991 & Not described & Not described \\
\hline & Yang 1991 & 0 & 0 \\
\hline \multirow{8}{*}{$\begin{array}{l}\text { Different durations of fluoro- } \\
\text { quinolones }\end{array}$} & Alam 1995 & 0 & 0 \\
\hline & Duong 1995 & 0 & 0 \\
\hline & Kalo 1997 & 0 & 0 \\
\hline & Nguyen 1997 & 0 & 0 \\
\hline & Tran 1995 & 0 & 0 \\
\hline & Unal 1996 & 0 & 0 \\
\hline & Vinh 1996 & 0 & 0 \\
\hline & Vinh 2005 & 0 & 0 \\
\hline
\end{tabular}


${ }^{a}$ Only trials reporting on serious adverse events are included.

${ }^{b}$ Zero (0) events only when specifically stated by trial author.

\section{Funnel plot}

We generated a funnel plot for clinical failure, microbiological failure, and relapse for the comparison of fluoroquinolone versus chloramphenicol, as there were more than five trials in these comparisons. No asymmetry was detected for the outcome clinical failure (Figure 3), while asymmetry was detected for microbiological failure (Figure 4) and relapse (Figure 5). However, the number of trials was very limited and well below the recommended number (10 trials) for meaningful interpretation.

Figure 3. Funnel plot to assess publication bias in outcome clinical failure for comparison of fluoroquinolones vs chloramphenicol

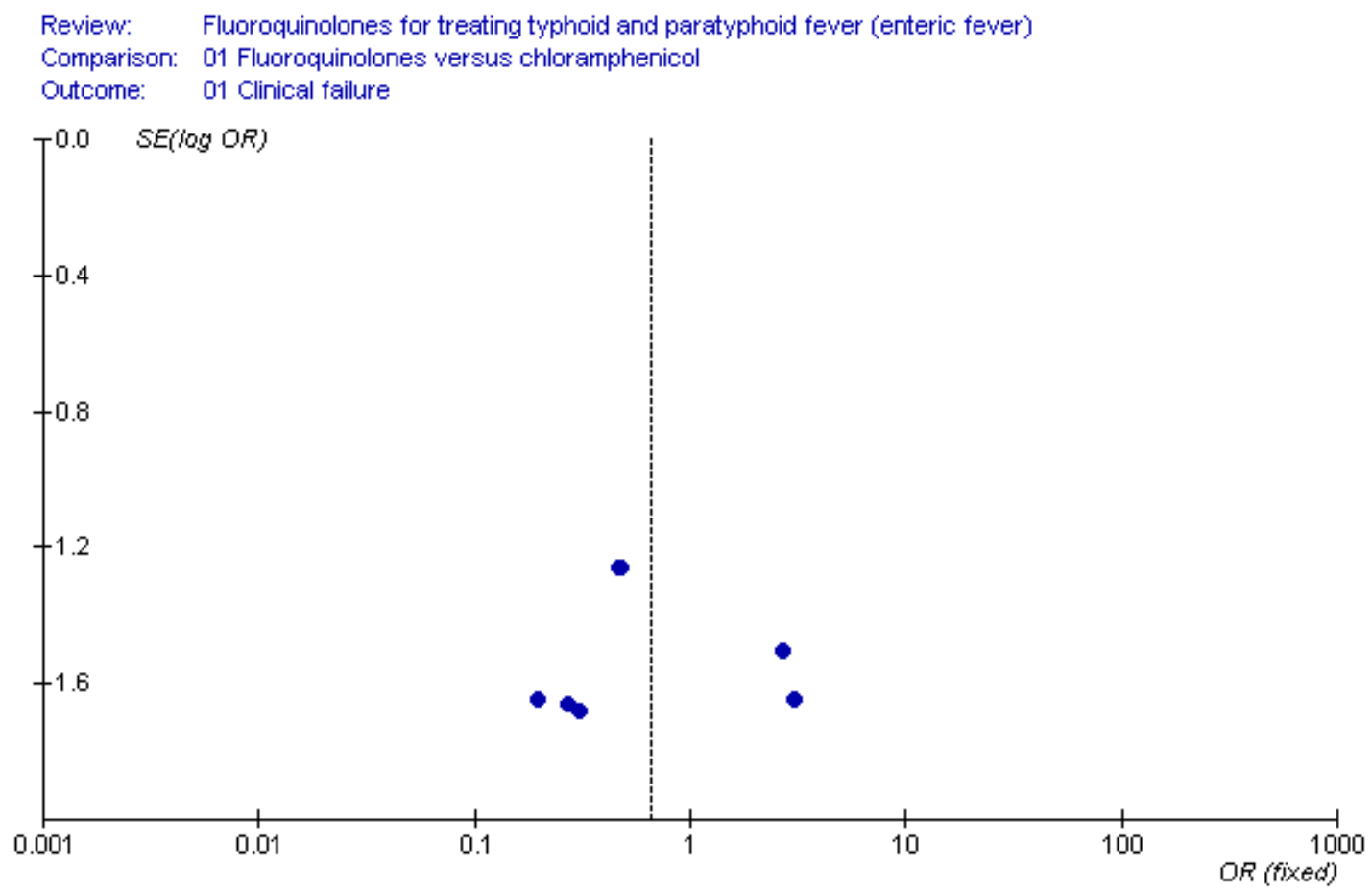


Figure 4. Funnel plot to assess publication bias in outcome microbiological failure for comparison of fluoroquinolones vs chloramphenicol

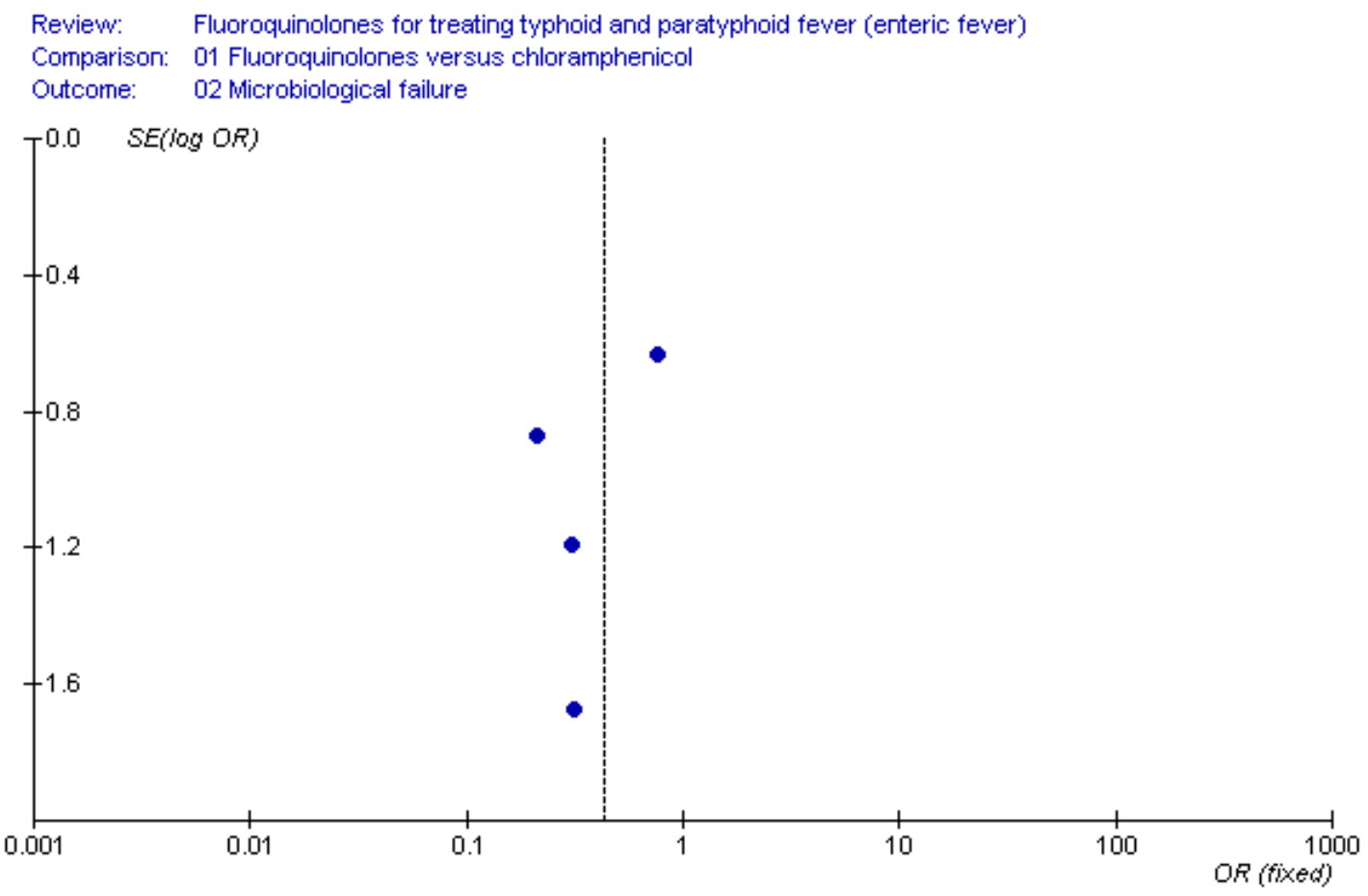

Fluoroquinolones for treating typhoid and paratyphoid fever (enteric fever) (Review) 
Figure 5. Funnel plot to assess publication bias in outcome relapse for comparison of fluoroquinolones vs chloramphenicol

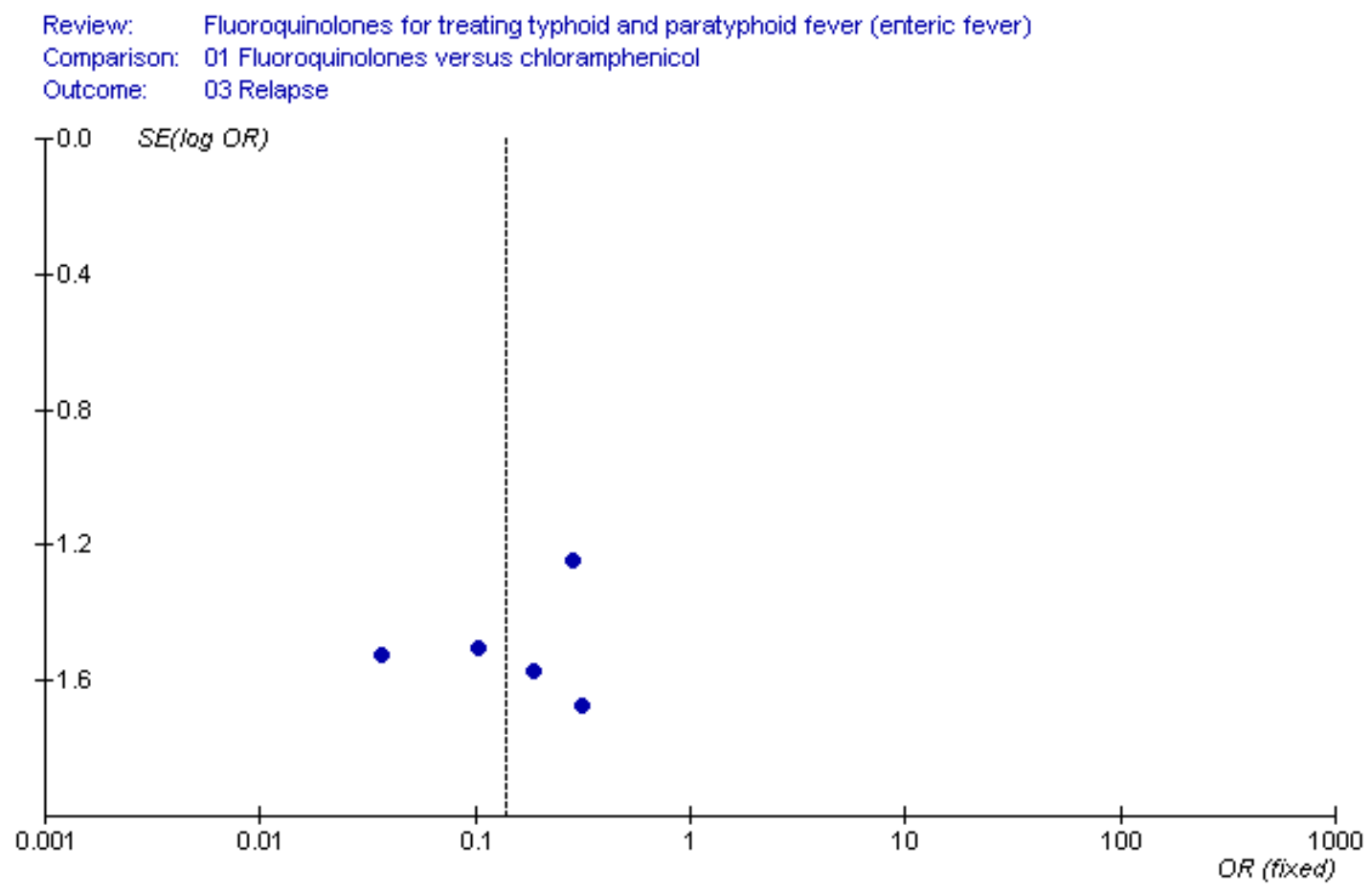

\section{I.2. Fluoroquinolones versus amoxicillin or ampicillin}

Two trials, both with an unknown proportion of participants with $\mathrm{MDR}$ and $\mathrm{NaR}$ strains, compared a fluoroquinolone with amoxicillin (Yousaf 1992) or ampicillin (Flores 1994, only abstract available).

\section{Clinical and microbiological failure, and adverse events}

All results for measured outcomes were statistically significant in favour of the fluoroquinolones - clinical failure (OR 0.08, 95\% CI 0.01 to 0.46 ; 90 participants, 2 trials, Analysis 2.1), microbiological failure (OR $0.10,95 \%$ CI 0.02 to 0.58 ; 90 participants, 2 trials, Analysis 2.2), and non-serious adverse events, described as mostly diarrhoea and rashes (OR $0.17,95 \%$ CI 0.04 to $0.73 ; 50$ participants, 1 trial, Analysis 2.3 and Table 6) - but the numbers of participants were very small so that confidence intervals were very wide.

\subsection{Fluoroquinolones versus co-trimoxazole}

Hajji 1988 and Limson 1989 compared pefloxacin and ciprofloxacin respectively with co-trimoxazole. Neither trial included participants with MDR strains, and only Hajji 1988 specifically reported the absence of $\mathrm{NaR}$ strains in the participants.

\section{Clinical and microbiological failure}

There was a trend favouring fluoroquinolones over co-trimoxazole for clinical failure (82 participants, 2 trials, Analysis 3.1) and microbiological failure (82 participants, 2 trials, Analysis 3.2), but we did not find any statistically significant results for these outcomes, although statistical power was extremely low so that confidence intervals were very wide.

\section{Fever clearance time}

Only Hajji 1988 reported on fever clearance time, which was 104.88 hours (mean) for the fluoroquinolone group compared with 186.00 hours (mean) for the co-trimoxazole group (Student's $t$ test; P value $<0.01$; no standard deviation reported). 


\section{Relapse and convalescent faecal carriers}

Hajji 1988 reported no relapses or convalescent faecal carriers.

\section{Adverse events}

We did not detect any statistically significant differences in nonserious adverse events, such as rash, nausea, abdominal discomfort, and dizziness (82 participants, 2 trials, Analysis 3.3; see Table 6). Hajji 1988 reported no serious adverse events.

\section{Fluoroquinolones versus second-line antibiotics (azithromycin, cefixime, and ceftriaxone)}

\section{I. Fluoroquinolones versus azithromycin: adults}

Girgis 1999 and Chinh 2000 compared ofloxacin and ciprofloxacin, respectively, with azithromycin. Chinh 2000 reported that almost half of the participants were infected with $\mathrm{NaR}$ strains, while Girgis 1999 did not test for NaR. We observed a tendency for azithromycin to perform better than fluoroquinolones for all outcomes except fever clearance times, but the sample size was very small and confidence intervals were very wide. Thus we cannot exclude chance as an explanation for these findings.

\section{Clinical and microbiological failure}

We did not detect any statistically significant differences in clinical failure (152 participants, 2 trials, Analysis 4.1) or microbiological failure (152 participants, 2 trials, Analysis 4.2). Out of the six clinical failures in the fluoroquinolone arm in Chinh 2000, four were infected with $\mathrm{NaR}$ strains, which could possibly explain the trend favouring azithromycin for clinical failure.

\section{Relapse}

We did not detect any statistically significant differences in relapse (102 participants, 2 trials, Analysis 4.3).

\section{Fever clearance time}

We also did not find any statistically significant difference in fever clearance time (152 participants, 2 trials, Analysis 4.4). The higher proportion of $\mathrm{NaR}$ strains in Chinh 2000 could explain the heterogeneity observed between the two trials.

\section{Length of hospital stay}

We did not detect any statistically significant difference in the length of hospital stay (152 participants, Analysis 4.5).

\section{Cost of therapy}

We did not detect any statistically significant difference in the cost of therapy (mean US\$ 28 (standard deviation (SD) 0) for fluoroquinolone group and mean US\$35 (SD 0) for azithromycin group).

\section{Convalescent faecal carriage}

We detected a statistically significant increase in the odds of convalescent faecal carriage in the fluoroquinolone group (OR 21.33, 95\% CI 1.18 to 386.00; 133 participants, 2 trials, Analysis 4.6), but the outcome was measured very early, at days two to three after the end of treatment, and the confidence interval is wide.

\section{Complications and adverse events}

We did not detect any statistically significant difference in complications (see Table 2 for details), as only one participant in each arm in Chinh 2000 had gastrointestinal bleeding (152 participants, 2 trials, Analysis 4.7). Non-serious adverse events included rashes and gastrointestinal symptoms, such as vomiting, abdominal pain, and diarrhoea or constipation, but the trialists did not report the number of participants with these events (Table 6); there were no serious adverse events.

\subsection{Fluoroquinolones versus azithromycin: children}

Two trials made this comparison: Parry 2007 had $98 \%$ NaR strains in the fluoroquinolone arm; and Dolecek 2008 had 96\% NaR strains in the fluoroquinolone arm. However, the fluoroquinolone used in Dolecek 2008 was a new-generation fluoroquinolone, gatifloxacin, which is active against $\mathrm{NaR}$ strains; thus the two trial were not combined. In both trials more than $60 \%$ of participants were children.

\section{Clinical and microbiological failure}

Parry 2007: We found a statistically significant increase in odds of clinical failure with fluoroquinolone (OR 2.67, 95\% CI1.16 to 6.11; 125 participants, 1 trial, Analysis 4.8). We did not detect any statistically significant difference in microbiological failure (125 participants, 1 trial, Analysis 4.9).

Dolecek 2008: We did not detect any statistically significant differences in clinical failure (285 participants, Analysis 4.8) or microbiological failure (285 participants, Analysis 4.9).

\section{Relapse}

Parry 2007: There were no relapses among participants seen at one month's follow up (114 participants followed from among 130 participants with culture-confirmed enteric fever, or less than $90 \%$ follow up at month for two included arms of the trial).

Dolecek 2008: We did not detect a statistically significant difference in relapse (264 participants, Analysis 4.10). 


\section{Fever clearance time}

Parry 2007: There was a statistically significant increase in fever clearance time (MD 57.60 hours, 95\% CI 28.31 to 86.89; 125 participants, Analysis 4.11).

Dolecek 2008: We did not detect a statistically significant difference in fever clearance time (285 participants, Analysis 4.11).

\section{Length of hospital stay}

Parry 2007: There was a borderline statistically significant increase in the length of hospital stay in the fluoroquinolone group (MD 1.10 days, $95 \%$ CI 0.00 to 2.20; 125 participants, Analysis 4.12). Dolecek 2008: We did not detect a statistically significant difference in length of hospital stay (285 participants, Analysis 4.12).

\section{Convalescent faecal carriage}

Parry 2007: We found a statistically significant increase convalescent faecal carriage in the fluoroquinolone group (OR 14.64, 95\% CI 1.84 to 116.48 ; 124 participants, 1 trial, Analysis 4.13).

Dolecek 2008: We did not detect a statistically significant difference in convalescent faecal carriage (268 participants, Analysis 4.13).

\section{Complications and adverse events}

Parry 2007: We did not detect any statistically significant difference in complications, which included gastrointestinal bleeding (125 participants, Analysis 4.14; see Table 2). Non-serious adverse events such as gastrointestinal and temporary joint symptoms were reported (numbers for gastrointestinal symptoms not reported, see Table 6). No serious adverse events were reported.

Dolecek 2008: There was a statistically significant decrease in complications in the fluoroquinolone group (OR 0.05, 95\% CI 0.00 to $0.94 ; 285$ participants, Analysis 4.14). We did not detect any statistically significant differences in non-serious adverse events (285 participants, Analysis 4.15). There were no serious adverse events.

\subsection{Fluoroquinolones versus cefixime: adults}

Two trials of adults, or mostly adults (Yu 1998; Pandit 2007) compared a fluoroquinolone with cefixime. Although one trial ( Pandit 2007) did have a high proportion of NaR strains, the newer fluoroquinolone used, gatifloxacin, is reported to be active against $\mathrm{NaR}$ strains; the other trial on adults (Yu 1998) did not provide any data for $\mathrm{NaR}$ strains. These two trials were combined in a metaanalysis. Pandit 2007 was an outpatient trial, where community medical auxiliaries conducted twice daily home-based assessments and provided directly observed treatment with study drugs; all participants were also seen at the hospital on day 10 .

\section{Clinical and microbiological failure}

We detected a statistically significant decrease in odds of clinical failure in the fluoroquinolone group (OR 0.05, 95\% CI 0.01 to 0.24; 238 participants, 2 trials, Analysis 5.1, Figure 6), and no statistically significant difference in microbiological failure $(238$ participants, Analysis 5.2).

Figure 6. Fluoroquinolones vs cefixime: clinical failure (adults or mostly adults)

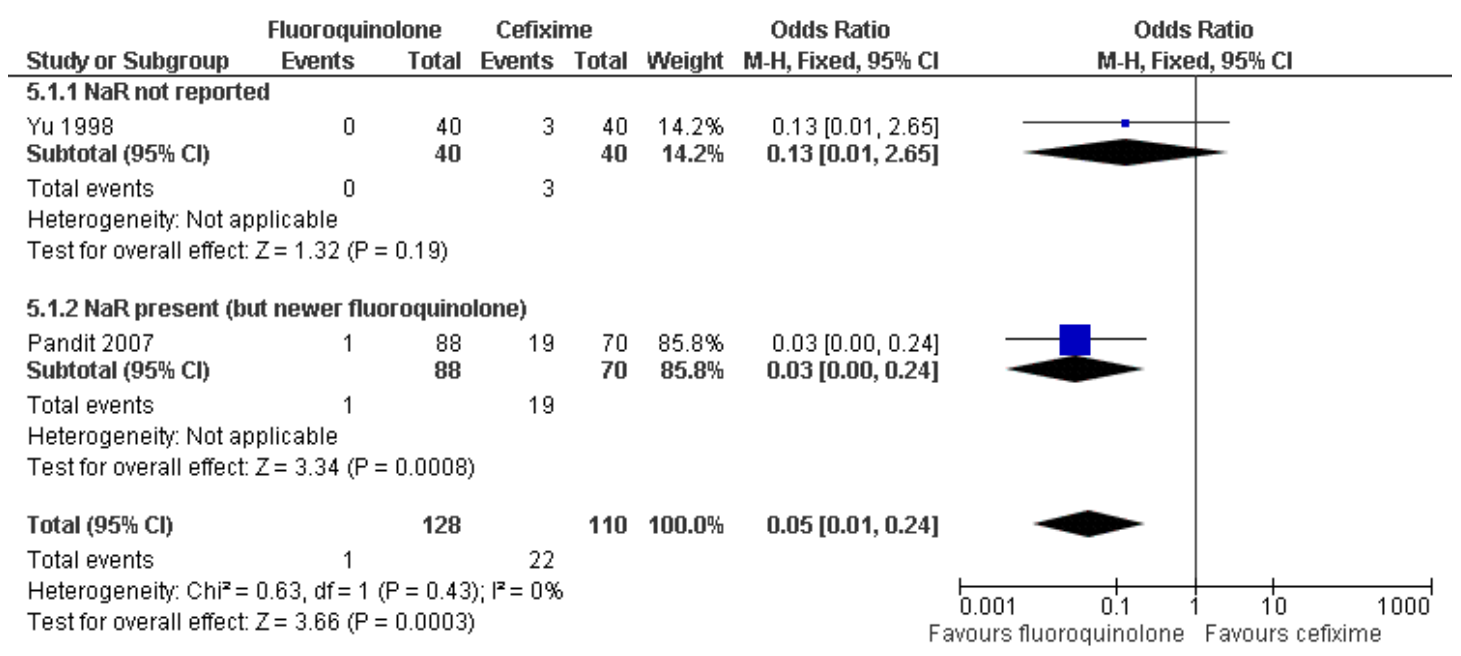

Fluoroquinolones for treating typhoid and paratyphoid fever (enteric fever) (Review) 


\section{Relapse}

There was a statistically significant reduction in relapse with the fluoroquinolone (OR 0.18, 95\% CI 0.03 to $0.91 ; 218$ participants, 2 trials, Analysis 5.3, Figure 7).

Figure 7. Fluoroquinolones vs cefixime: relapse (adults or mostly adults)

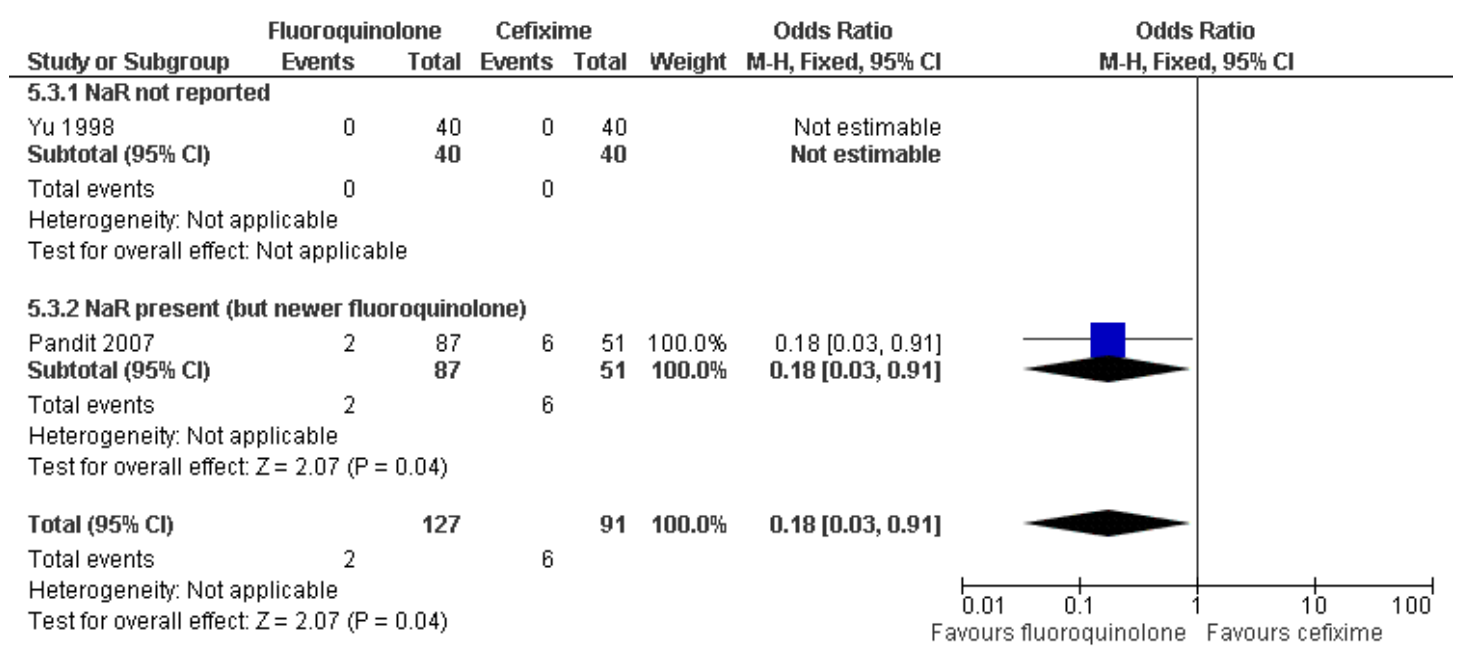

\section{Fever clearance time}

We detected a statistically significant reduction in fever clearance time with the fluoroquinolone (MD - 41.69 hours, 95\% CI -54.96 to -28.42 ; 238 participants, 2 trials, Analysis 5.4).

\section{Cost of therapy}

The estimated cost of treatment provided in one trial was US\$ 1.2 for seven days of gatifloxacin and US\$ 12 for seven days of cefixime (generic drugs manufactured in India) (Pandit 2007).

\section{Convalescent faecal carriers}

We did not find any statistically significant difference in convalescent faecal carriers (227 participants, 2 trials, Analysis 5.5).

\section{Complications and adverse events}

There was no statistically significant difference for complications, which included one death in the cefixime group (158 participants,
1 trial, Analysis 5.6; see Table 2), and no serious adverse events (158 participants, 1 trial).

We found statistically significant heterogeneity in trials comparing non-serious adverse events (Analysis 5.7). We did not find any statistically significant difference in the one included trial (80 participants), but we found a statistically significant increase in the odds of nausea and vomiting in the other trial (OR 17.74, 95\% CI 2.30 to $136.58 ; 158$ participants; see Table 6). The heterogeneity could be attributed to the different fluoroquinolones used in the two trials (the relatively newer, gatifloxacin and levofloxacin). We did not find any statistically significant difference in non-serious adverse events when we used a random-effects model to combine the trials (OR 3.30, 95\% CI 0.11 to $97.30 ; 238$ participants, 2 trials).

\subsection{Fluoroquinolones versus cefixime: children}

One trial, Cao 1999, compared a fluoroquinolone with cefixime in children; no NaR strains were reported. The results for all measured outcomes favoured fluoroquinolones, but the sample sizes were small and hence the confidence intervals were very wide. 


\section{Clinical and microbiological failure}

We did not detect any statistically significant difference in clinical failure (82 participants, Analysis 5.8) or microbiological failure (82 participants, Analysis 5.9).

\section{Relapse}

We did not detect any statistically significant difference in relapse (40 participants, Analysis 5.10).

\section{Fever clearance time}

There was a statistically significant reduction in fever clearance time in the fluoroquinolone group (MD -91.00 hours, 95\% CI 115.89 to $-66.11 ; 78$ participants, Analysis 5.11).

\section{Length of hospital stay}

We found a statistically significant reduction in the length of hospital stay in the fluoroquinolone group (MD -3.00 days, 95\% CI -4.53 to $-1.47 ; 81$ participants, Analysis 5.12).

\section{Convalescent faecal carriers}

No convalescent faecal carriers were reported.

\section{Complications and adverse events}

There was no statistically significant difference in complications (82 participants, Analysis 5.13), which included one death and one child with gastrointestinal bleeding in the fluoroquinolone arm, and one child requiring blood transfusion in the cefixime arm (see Table 2). Non-serious adverse events included gastrointestinal symptoms, such as abdominal pain (see Table 6), but the number of participants was not reported.

\subsection{Fluoroquinolones versus ceftriaxone}

Three trials compared a fluoroquinolone with ceftriaxone protocol (Wallace 1993; Smith 1994; Tran 1994). There were no NaR strains in Smith 1994, but this information was unavailable for Tran 1994 and Wallace 1993. The stratifications by NaR strains were not meaningful due to the limited number of trials in the comparison.

\section{Clinical and microbiological failure}

The odds of clinical failure were reduced by $92 \%$ in the fluoroquinolone group (OR $0.08,95 \%$ CI 0.01 to 0.45 ; 120 participants, 3 trials, Analysis 6.1, Figure 8). We did not detect a statistically significant difference in the odds of microbiological failure (119 participants, 3 trials, Analysis 6.2), but sample sizes were small and confidence intervals were wide.

Figure 8. Fluoroquinolones vs ceftriaxone: clinical failure

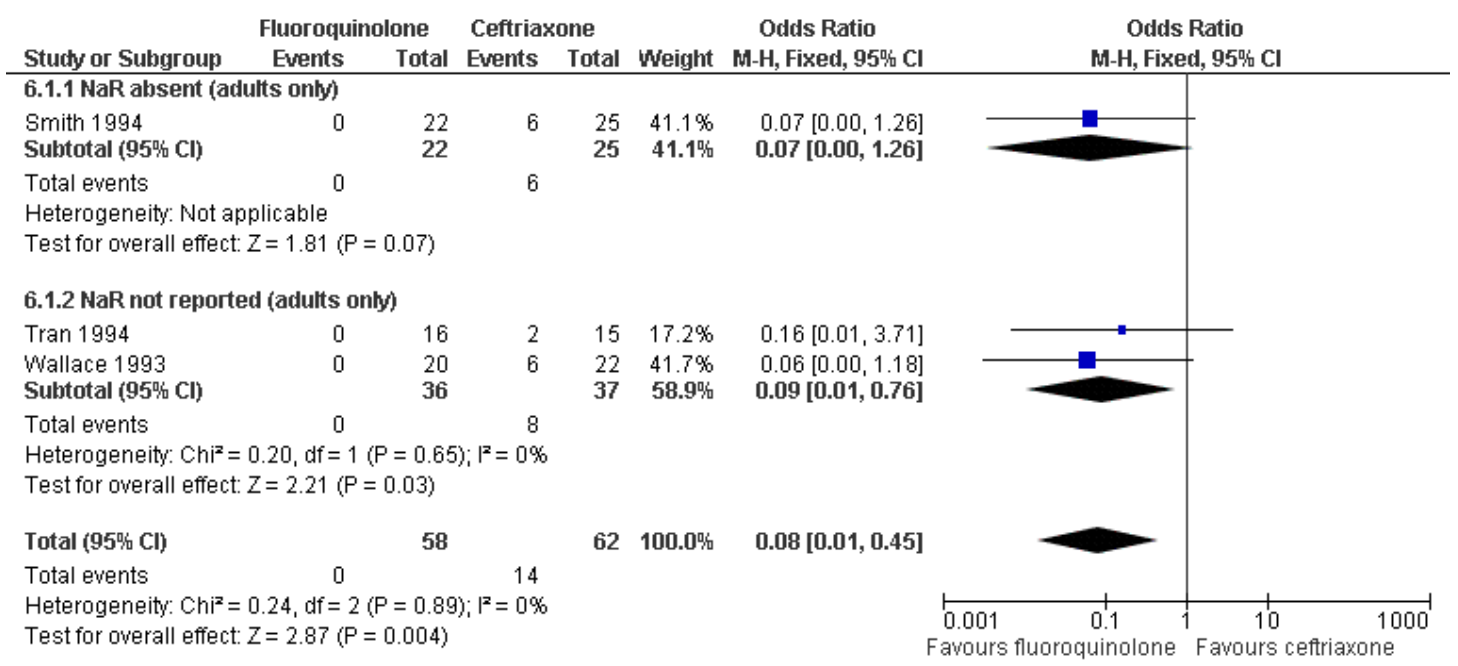




\section{Relapse}

We did not detect a statistically significant difference in the odds of relapse ( 81 participants, 3 trials, Analysis 6.3), but sample sizes were small and confidence intervals were wide.

\section{Fever clearance time}

Fever clearance times were statistically significantly lower in the fluoroquinolone group (MD -101.20 hours, 95\% CI -129.21 to 73.19; 76 participants, 2 trials, Analysis 6.4). Tran 1994 excluded two clinical failures in the ceftriaxone group when calculating mean fever clearance time, which may result in an underestimate of the difference in this outcome.

\section{Length of hospital stay}

Smith 1994 reported the on length of hospital stay as a mean of nine days (range 6 to 13 days) in the ofloxacin group and a mean of 12 days (range 7 to 23 days) in the ceftriaxone group; standard deviation was not reported $(\mathrm{P}<0.01)$.

\section{Convalescent faecal carriage}

We did not detect any significant difference for convalescent faecal carriage (81 participants, 3 trials, Analysis 6.5).

\section{Complications and adverse events}

There was no significant difference in the number of complications, including anaemia, jaundice (120 participants, 3 trials, Analysis 6.6; see Table 2), serious adverse events - one case of anaphylaxis in the ceftriaxone group (78 participants, 2 trials, Analysis 6.7; see Table 7), or non-serious adverse events, which included skin rash and pruritis (47 participants, 1 trial, Analysis 6.8; see Table 6); however, sample sizes were very small so confidence intervals were very wide.

\section{Norfloxacin trials}

We analysed norfloxacin trials separately from other fluoroquinolone trials because the WHO does not recommend this fluoroquinolone for treating enteric fever (WHO 2003).

\section{I. Norfloxacin versus chloramphenicol}

Only one of three trials reported the proportion of participants with $\mathrm{NaR}$ strains (all strains were resistant to norfloxacin) (Sarma 1991). MDR strains were present in Sarma 1991, not reported in Nalin 1987, and absent in Morelli 1992. In Sarma 1991 all eight MDR strains appeared in the norfloxacin group, and only 40 participants were randomized in all.

\section{Clinical and microbiological failure}

There was a statistically significant increase in the odds of clinical failure in the norfloxacin group among all trials (OR 5.80, 95\% CI 1.87 to $17.98 ; 259$ participants, Analysis 7.1); the one trial that did not report the proportion of MDR or NaR showed no statistically significant difference (169 participants, Nalin 1987).
We did not detect a statistically significant difference in the odds of microbiological failure (209 participants, 2 trials, Analysis 7.2).

\section{Relapse}

We did not detect a statistically significant difference in the odds of relapse (90 participants, 2 trials, Analysis 7.3).

\section{Fever clearance time}

For fever clearance time (Analysis 7.4), we observed marked heterogeneity between Sarma 1991 and Nalin 1987, which could be explained by the different definitions of fever clearance time used (see Table 3), thus we did not combine the trials. Sarma 1991, which also had some participants with MDR strains, showed that the fever clearance time was statistically significantly lower in the norfloxacin group (MD -36.00 hours, 95\% CI -44.77 to -27.23; 40 participants), while Nalin 1987 showed that it was statistically significantly higher in the norfloxacin group (MD 38.40 hours, 95\% CI 23.08 to $53.72 ; 169$ participants).

\section{Length of hospital stay}

The length of hospital stay was uniform (14 days) in all participants included (Analysis 7.5).

\section{Convalescent faecal carriage}

We did not detect a statistically significant difference for convalescent faecal carriage (259 participants, 3 trials, Analysis 7.6).

\section{Adverse events}

We did not detect a statistically significant difference for nonserious adverse events, such as gastrointestinal symptoms (Table 6 ), in the norfloxacin group and decreased blood cell counts in the chloramphenicol group (209 participants, 2 trials, Analysis 7.7).

\subsection{Norfloxacin versus ceftriaxone}

One trial involving children, and which did not report the proportion of participants with $\mathrm{NaR}$ strains, made this comparison ( Huai 2000).

\section{Clinical failure}

There was insufficient statistical power to detect a statistically significant difference in clinical failure between the two treatment groups (60 participants, Analysis 8.1).

\section{Relapse}

There was insufficient statistical power to detect a statistically significant difference in relapse between the two treatment groups (60 participants, Analysis 8.2).

\section{Fever clearance time}

The fever clearance time was statistically significantly higher in the norfloxacin group (MD 48 hours, 95\% CI 30.82 to 65.18 ; 60 participants, Analysis 8.3). 


\section{Adverse events}

We did not find a statistically significant difference for non-serious adverse events, which were all gastrointestinal (60 participants, Analysis 8.4; see Table 6).

\subsection{Norfloxacin versus other fluoroquinolones}

Three trials compared norfloxacin with pefloxacin (three trials), ofloxacin (three trials, of which two did not state the proportion of children included in the trials (Xiao 1991; Yang 1991)), or enoxacin (two trials).

\section{Clinical failure}

The odds of clinical failure (Analysis 9.1) increased with norfloxacin compared with pefloxacin (OR 30.60, 95\% CI 5.75 to 162.86; 200 participants, 3 trials), ofloxacin (OR 28.15, 95\% CI 4.80 to $165.14 ; 123$ participants, 3 trials), and enoxacin (OR 4.15, 95\% CI 1.77 to 9.76 ; 142 participants, 2 trials).

\section{Relapse}

No relapses were reported for norfloxacin and pefloxacin or enoxacin, and we did not detect a statistically significant difference between ofloxacin and norfloxacin (106 participants, 2 trials, Analysis 9.2).

\section{Fever clearance time}

The fever clearance time (Analysis 9.3) was statistically significantly longer in the norfloxacin groups compared with the pefloxacin group (MD 18.83 hours, 95\% CI 2.62 to 35.03; 144 participants, 2 trials) and enoxacin group (MD 60.00 hours, $95 \%$ CI 33.81 to $86.19 ; 102$ participants, 1 trial). It was also longer compared with ofloxacin in Yang 1991 (MD 69.60 hours, 95\% CI 42.03 to 97.17 ; 56 participants), but not in Xiao 1991 (MD 12.00 hours, $95 \%$ CI -40.58 to $16.58 ; 17$ participants). However, the mean difference for Xiao 1991, which contributed data to the pefloxacin and ofloxacin comparisons, could have been underestimated because it was unclear whether the trialists had excluded clinical failures when calculating the mean fever clearance time.

\section{Convalescent faecal carriage}

We did not detect any statistically significant difference in convalescent faecal carriage (Analysis 9.4) between norfloxacin and pefloxacin (56 participants, 1 trial), ofloxacin (106 participants, 2 trials), and enoxacin (40 participants, 1 trial).

\section{Adverse events}

We did not detect a statistically significant difference in the number of non-serious adverse events (Analysis 9.5), mainly skin rashes (see Table 6), between norfloxacin and ofloxacin (56 participants, 1 trial) or enoxacin (102 participants, 1 trial).

\section{Different fluoroquinolone durations}

4.I. Fluoroquinolones for 2 days versus 3 days
One trial in adults (Nguyen 1997) and two in children (Vinh 1996; Vinh 2005) made this comparison. Although all three trials reported the percentage of participants with $\mathrm{NaR}$ strains $-2.5 \%$ (Vinh 2005), 5\% (Nguyen 1997), and 13\% (Vinh 1996) - we were unable to determine its impact on the results because there were so few trials.

\section{Adults}

We did not detect a statistically significant difference in clinical failure (100 participants, Analysis 10.1), relapse (50 participants, Analysis 10.3), fever clearance time (100 participants, Analysis 10.4), length of hospital stay (100 participants, Analysis 10.5), or complications (gastrointestinal bleeding, jaundice, and hypotension in the 3-day arm) (100 participants, Analysis 10.7; see Table 2). There were no microbiological failures or convalescent faecal carriers.

\section{Children}

We did not detect a statistically significant difference in clinical failure (296 participants, 2 trials, Analysis 10.1), microbiological failure (296 participants, 2 trials, Analysis 10.2), relapse (262 participants, 2 trials, Analysis 10.3), fever clearance time (296 participants, 2 trials, Analysis 10.4), length of hospital stay (296 participants, 2 trials, Analysis 10.5), convalescent faecal carriage (262 participants, 2 trials, Analysis 10.6), complications (gastrointestinal bleeding and delirium (296 participants, 2 trials, Analysis 10.7; see Table 2), or non-serious adverse events (urticaria in 3-day arm) (296 participants, Analysis 10.8; see Table 6). There were no serious adverse events.

\subsection{Fluoroquinolones for 3 days versus 5 days}

Two trials - one with over 70\% children (Tran 1995) and one on adults (Duong 1995) - compared three days with five days of ofloxacin and fleroxacin, respectively. Duong 1995 did not perform NaR testing, while some participants in Tran 1995 had NaR strains, although the precise number of these participants was not available.

Adults

We did not detect a statistically significant difference in clinical failure (63 participants, Analysis 11.1), relapse (40 participants, Analysis 11.2), fever clearance time (663 participants, Analysis 11.3), or length of hospital stay (63 participants, Analysis 11.4). There were no microbiological failures, complications, or serious adverse events in either arm.

\section{Children}

We did not detect a statistically significant difference in relapse (154 participants, Analysis 11.2). Fever clearance time was statistically significantly lower in the three-day group (MD -12.00 hours, 95\% CI -18.07 to -5.93; 195 participants, Analysis 11.3). There were no statistically significant differences in non-serious 
adverse events, such as insomnia, headache, nausea, vomiting and diarrhoea (228 participants, Analysis 11.5). There were no clinical failures, microbiological failures, convalescent faecal carriers, or serious adverse events in either arm.

\subsection{Fluoroquinolones for 5 days versus 7 days}

One trial, which did not report the proportion of participants with $\mathrm{NaR}$ strains, made this comparison (Unal 1996). There were no clinical failures in either arm, and we did not detect a statistically significant difference in microbiological failure (46 participants, Analysis 12.1), relapse (46 participants, Analysis 12.2), fever clearance time (46 participants, Analysis 12.3), or non-serious adverse events (46 participants, Analysis 12.4; see Table 6).

\section{Fluoroquinolone for 7 days versus 10 or 14 days}

We defined short-course treatment as seven or less days and longcourse treatment as more than seven days. One trial compared perfloxacin for 7 days with 10 days (Kalo 1997), and a multicenter trial compared fleroxacin for 7 days with 14 days (Arnold 1993). Neither trial reported the proportion of participants with $\mathrm{NaR}$ strains. We did not detect a statistically significant difference in microbiological failure (87 participants, 2 trials, Analysis 13.1) or relapse ( 87 participants, 2 trials, Analysis 13.2). There were no clinical failures or convalescent faecal carriers.

\section{Fluoroquinolones for 10 days versus 14 days}

One trial, with seven per cent of the participants with $\mathrm{NaR}$ strains, made this comparison (Alam 1995). We did not detect a statistically significant difference in relapse (69 participants, Analysis 14.1), fever clearance time (69 participants, Analysis 14.2), or nonserious adverse events (gastrointestinal symptoms, headache and rashes in both arms, and one case of joint pain in the 14-day arm (69 participants, Analysis 14.3; see Table 6). There were no clinical or microbiological failures, or convalescent faecal carriers.

\section{ISCUSSION}

Even though in endemic areas enteric fever most commonly affects children, this review demonstrates the paucity of data from adequately designed randomized controlled trials in children.

\section{Limitations in analysis and interpretation}

The sample sizes in each trial, as well as the number of trials in each comparison, were very small. The pooled sample sizes were also very small, thus there was very little statistical power, and we cannot exclude chance as an explanation for results of many comparisons. The methodological quality and the quality of reporting of the trials were variable and sometimes poor. The method of allocation concealment was unclear in 22 trials, and the method of randomization was unclear in 17 of the 38 trials, meaning they were potentially open to selection bias. We could not perform meaningful sensitivity analyses excluding trials with poor methodological quality due to the small number of trials in each comparison, except for a limited number of comparisons of fluoroquinolones with chloramphenicol.

The small number of trials also precluded the meaningful use of a funnel plot (assessment of publication bias). In the funnel plots generated for primary outcomes for fluoroquinolones versus chloramphenicol, no asymmetry was found for clinical failure, but asymmetry was detected for microbiological failure and relapse. Although interpretation is extremely limited due to the limited number of trials, the asymmetry could indicate the failure to publish smaller trials that did not show any statistically significant difference between older drugs and fluoroquinolones (Stern 1997). However, we conducted a thorough search for trials and also identified an additional two ongoing trials, which we will include in a future update.

Another factor limiting these analyses and interpretation was the lack of explicit definitions of outcomes measured in some trials, especially for relapse, and wide variations in the times at which the outcomes were measured, particularly for microbiological failure and relapse. Some trials did not clearly report whether symptomatic relapse was confirmed by a positive blood culture. Resistance data were also not explicitly reported, particularly in older trials. Four of the 13 trials that compared fluoroquinolones with a first-line agent (chloramphenicol, co-trimoxazole, and ampicillin or amoxicillin) did not report the proportion of participants with MDR strains, and only 13 of 38 trials reported $\mathrm{NaR}$ data.

Most of the trials did not explicitly report the number of participants included when measuring fever clearance time. The mean fever clearance times may also be skewed, which means some participants take longer times to clear fever due to a variety of reasons, and a meta-analysis of such arithmetic means may not be entirely accurate. The persistence of fever despite clearance of $S$. Typhi and $S$. Paratyphi from the bloodstream has been attributed to the continued production of pyrogenic cytokines (Islam 1988; Lasserre 1991; Bhutta 1994; Acharya 1995) and may also not be an adequate indicator of antibiotic efficacy.

We did not observe statistically significant heterogeneity in the included trials with the exception of those involving comparisons with norfloxacin and for selected secondary outcomes among some trials, such as in non-serious adverse events with use of a newer fluoroquinolone (gatifloxacin), and length of hospitalization for fluoroquinolones versus chloramphenicol. For the norfloxacin trials, we were unable to explain the observed heterogeneity due to the small number of trials, although the WHO does not recommend norfloxacin for treating enteric fever because of poor oral bioavailability (WHO 2003). Gatifloxacin is a relatively new drug; an ongoing trial of gatifloxacin in enteric fever was temporarily stopped due to safety concerns (ISRCTN53258327).

\section{Applicability}


These meta-analyses included only blood or bone marrow cultureconfirmed cases of enteric fever, whereas in most endemic areas, enteric fever is treated on the basis of clinical suspicion without confirmation by culture, owing to the absence of culture facilities. Most trials included adult inpatients. Inpatients represent the severe end of the spectrum of enteric fever. In endemic countries, up to $90 \%$ of cases of enteric fever are managed safely in the outpatient setting (Parry 2002). Also, children differ from adults with enteric fever in terms of disease presentation, severity, and complications (Butler 1991; Mahle 1993; Walia 2006). Another factor that may limit applicability in children is the relatively limited data regarding fluoroquinolone pharmacokinetics and adverse effects in this age group (Gendrel 2003; Committee 2006). Thus data obtained from adult inpatients have limited applicability to situations in many parts of the developing world where the vast majority of cases of enteric fever are in children and most of which are treated in the outpatient setting.

The use of fluoroquinolones as first-line antibiotics in such settings has resulted in gross overuse of these antibiotics and high levels of resistance to fluoroquinolone have emerged rapidly in $S$. Typhi and $S$. Paratyphi in countries where fluoroquinolones are used as firstline antibiotics (Biswal 1994; Brown 1994; Rowe 1995; Murdoch 1998; Chandel 2000; Chandel 2001; Threlfall 2001; Butt 2003; Threlfall 2003; Karunanayake 2004; Slinger 2004; Butt 2005; Manchanda 2006; Mohanty 2006; Walia 2006; Chau 2007; Joshi 2007).

\section{Impact of resistance strains}

The changing pattern of resistance, including rapidly rising resistance to fluoroquinolones, also affects the applicability of these results - particularly for older fluoroquinolones, such as ofloxacin. In one recent trial that included mainly children with a very high proportion of $\mathrm{NaR}$ strains, ofloxacin had significantly higher number of clinical failures (Parry 2007). We conducted sensitivity analyses to determine the impact of excluding trials with $\mathrm{NaR}$ or MDR strains, and those trials that did not report the proportion of participants with $\mathrm{NaR}$ or MDR strains. However, the analyses were mostly uninformative owing to lack of statistical power. Newer fluoroquinolones (such as gatifloxacin) are not affected by $\mathrm{NaR}$ strains (Pandit 2007; Dolecek 2008), and two trials with NaR strains but which used gatifloxacin were considered separately, where possible, from trials of older fluoroquinolones that reported $\mathrm{NaR}$ strains. More meaningful sensitivity analyses involving different proportions of strains with resistance to fluoroquinolones may be possible in future updates of this review.

\section{Fluoroquinolones versus first-line antibiotics (chloramphenicol, co-trimoxazole, and ampicillin or amoxicillin) in children}

We are unable to draw conclusions about the use of fluoroquinolones compared to first-line drugs in children as we did not find any trials involving these comparisons.

\section{Fluoroquinolones versus second-line drugs (azithromycin, ceftriaxone, and cefixime) in children}

There were very limited data for these comparisons, and thus no firm conclusions can be made. One small, open trial with adequate methods of randomization and allocation concealment compared fluoroquinolone with cefixime (Cao 1999). The trial showed that fluoroquinolones were not significantly different from cefixime for all the primary outcomes studied (clinical failure, microbiological failure, and relapse), although confidence intervals were very wide. Fluoroquinolones were significantly better than cefixime in reducing fever clearance times.

An open trial published in 2007, which had adequate methods of randomization and allocation concealment, involved mostly children, and had a high proportion of $\mathrm{NaR}$ strains, found that ofloxacin administered for seven days had significantly higher clinical failure and fever clearance times compared with azithromycin (Parry 2007). Another trial, published in 2008 (Dolecek 2008), which also had adequate methodological quality, found no statistically significant differences between a newer fluoroquinolone (gatifloxacin) and azithromycin, although confidence intervals were very wide.

One trial that could not be included in this review compared ofloxacin and ceftriaxone in children (Kumar 2007). The only results presented were mean fever clearance times, which were significantly different in the ofloxacin group compared to the ceftriaxone group ( 4.97 vs 4.26 days, $\mathrm{P}<0.05$ ); other details, such as the number of participants in each group and number of strains with reduced susceptibility to fluoroquinolones, were not specified.

\section{Fluoroquinolones versus first-line antibiotics (chloramphenicol, co-trimoxazole, and ampicillin or amoxicillin) in adults}

The sample sizes in these trials were very small and confidence intervals were very wide. Among 10 trials comparing fluoroquinolones with chloramphenicol, only two open trials reported using adequate methods of randomization and allocation concealment, and lost no participants during the short-term follow up ( Gasem 2003; Phongmany 2005). We found fluoroquinolones to be better than chloramphenicol for reducing the odds of relapse. However, most trials were also of low methodological quality and did not explicitly report the definition, or the culture site used to confirm symptomatic relapse, or both. We did not find any significant difference between fluoroquinolones and chloramphenicol for relapses clearly confirmed by blood cultures, although this was based on only one trial with low statistical power and low methodological quality. We found fluoroquinolones to be better in reducing fever clearance time and duration of hospitalization including in trials of adequate methodological quality. Clinical failure and microbiological failure were comparable between the two groups although the confidence intervals were wide. 
We did not detect a statistically significant difference between fluoroquinolones and co-trimoxazole for any measured outcome in adults, but statistical power was very low and only one of the two open trials had adequate method of randomization, as well as allocation concealment and follow up (Hajji 1988). We found fluoroquinolones to be significantly better than amoxicillin or ampicillin for clinical and microbiological failure. However, neither of the two included trials reported the proportion of participants with MDR strains. One trial report was an abstract with limited information (Flores 1994), and the other was of low methodological quality (an open trial that had inadequate randomization and allocation concealment, and included less than $90 \%$ of the participants in the analysis) (Yousaf 1992). Thus both may have been open to selection bias, which may have operated in favour of the newer fluoroquinolones.

\section{Fluoroquinolones versus second-line drugs (azithromycin, ceftriaxone, and cefixime) in adults}

Fluoroquinolones were not significantly different from azithromycin for any primary outcome in inpatients. However, the confidence intervals were wide because only two small trials contributed data, although both used adequate methods of randomization and allocation concealment. The higher number of clinical failures observed in the fluoroquinolone group in one of these trials, Chinh 2000 , could be due to a high proportion (> 50\%) of participants infected with $\mathrm{NaR}$ strains.

Fluoroquinolones were associated with a significant and large reduction in the odds of clinical failure and fever clearance time compared with ceftriaxone, based on three small open trials involving inpatients (Wallace 1993; Smith 1994; Tran 1994). Two of these trials used an adequate method of randomization and allocation concealment (Smith 1994; Tran 1994). We did not find a statistically significant difference between the antibiotics for any other measured outcomes, although confidence intervals were wide, and we could not assess the impact of $\mathrm{NaR}$ strains on these results meaningfully.

We found results in favour of fluoroquinolones for clinical failure and relapse as well as fever clearance time from two trials comparing cefixime and a fluoroquinolone (Pandit 2007; Yu 1998), although one was of low methodological quality and did not report $\mathrm{NaR}$ strains. One of these trials used a newer fluoroquinolone active against NaR strains (Pandit 2007).

\section{Comparison with past reviews}

The results of this systematic review differ from those of an earlier summary of 57 randomized controlled trials of enteric fever, 10 of which compared a fluoroquinolone with a non-fluoroquinolone antibiotic (Parry 2002). Parry 2002, which did not use meta-analytic techniques, reported that clinical failures and fever clearance times with fluoroquinolone therapy were lower compared with first-line antibiotics, ceftriaxone, and cefixime. In our meta-analyses of 23 randomized controlled trials comparing fluoroquinolones with different antibiotics and which separated trials on children and adults, we found most trials to be of low methodological quality and lacking in statistical power, with wide confidence intervals. Hence, no conclusive evidence of superiority of fluoroquinolones over first-line antibiotics (chloramphenicol, co-trimoxazole, and ampicillin or amoxicillin) could be made for clinical failure. There is, however, better evidence to suggest that fever clearance times are lower with fluoroquinolones compared to chloramphenicol in adults. For comparisons of fluoroquinolones with ceftriaxone and cefixime, as mentioned above, we found clinical failures to be lower and fever clearance times also shorter with fluoroquinolones. However, these results are based on few trials, including some of low methodological quality, and mainly on data from adults.

\section{Cost of therapy}

In this review, we could not compare the cost of fluoroquinolone therapy in relation to other antibiotics because all but Girgis 1999 and Pandit 2007 did not report these data. In most low-income countries, fluoroquinolones are available at a much higher cost than first-line antibiotics; for example, in Pakistan, a 10-day course of ciprofloxacin costs approximately 1.5 times (local brand) to up to five times (international brand) that of a conventional 14day course of chloramphenicol (retail prices of several brands in Karachi, Pakistan). The cost of a shorter (three-day) course of ciprofloxacin, however, ranges from less than half to 1.5 times greater than a 14-day chloramphenicol regimen; only one trial made this comparison (Phongmany 2005). Fluoroquinolones may be the least costly option for the treatment of MDR enteric fever compared with costs of azithromycin, cefixime, and ceftriaxone (retail prices of several brands in Karachi, Pakistan), but increasing numbers of clinical failures in trials with $\mathrm{NaR}$ strains with older fluoroquinolones (Chinh 2000; Parry 2007) suggest that this cost advantage of using older fluoroquinolones has been overwhelmed by declining efficacy in the face of rising resistance. Rising levels of resistance could in the near future also affect the efficacy of newer generation fluoroquinolones (Turner 2006).

\section{Different fluoroquinolones}

Among various fluoroquinolones, we found all three classes analysed (pefloxacin, ofloxacin, and enoxacin) to be significantly superior compared with norfloxacin for reducing clinical failure. These trials originated largely from China, and none specified the method of randomization, allocation concealment, blinding, or follow up, and thus we deemed them to be of low methodological quality.

\section{Different durations of fluoroquinolone therapy}

A large number of different durations were compared in several trials. The number of trials for each comparison was small, mostly with small sample sizes and hence lacking considerably in statistical power. Only two trials compared a short-course regimen (seven 
days or less) with a long-course regimen (more than seven days). Clinical failure, microbiological failure, and relapse rates were low in both arms, but the data were not sufficient to enable us to exclude chance as an explanation for these findings.

\section{Adverse events}

A serious adverse event was reported in three instances (anaphylaxis in ceftriaxone group, severe leucopenia in chloramphenicol group, and a rash in the ciprofloxacin group). Overall, few participants reported adverse events. These were mainly abdominal symptoms, such as nausea or vomiting, abdominal pain, or rashes; however, a trial involving a newer fluoroquinolone (gatifloxacin) reported a statistically significantly larger number of participants with nausea and vomiting when compared to the non-fluoroquinolone arm. Mild joint pain was reported in one case in a 14-day fluoroquinolone group. One child in each arm (fluoroquinolone and azithromycin arm) of a trial reported temporary joint discomfort. The maximum period of follow up was six months (two trials), thus most trials could not adequately address long-term adverse effects, particularly on growing joints.

\section{A U THORS, CONCLUSIONS}

\section{Implications for practice}

A lack of data precludes firm conclusions to be made regarding superiority of fluoroquinolones over first-line antibiotics (chloramphenicol, ampicillin, amoxicillin), cefixime, or ceftriaxone in children. Data from one trial suggest that azithromycin may be better than ofloxacin (an older fluoroquinolone) in children infected with a high proportion of strains with reduced susceptibility to fluoroquinolones. We did not find any statistically significant differences in primary outcomes in one trial of azithromycin and gatifloxacin (a new-generation fluoroquinolone) in children.

In adult inpatients, data suggest that fluoroquinolones may be better than chloramphenicol for reducing clinical relapse. Limited data from adults suggest that fluoroquinolones may also be better than ceftriaxone for reducing clinical failure, and may be better than cefixime for reducing clinical failure and relapse. We did not find any statistically significant differences in primary comparisons of azithromycin and older fluoroquinolones in adults.

No conclusions could be made for superiority of any particular duration of fluoroquinolone therapy.

No conclusions can be made regarding adverse effects in children, owing to the short length of follow up in most of these trials, and few trials involving children.

\section{Implications for research}

Appropriate therapy for enteric fever remains a clinical and public health dilemma. High prevalence of resistance to first-line antibiotics (MDR strains) and rapid emergence of resistance to fluoroquinolones among $S$. Typhi and $S$. Paratyphi have added to the complexity of this issue in resource-constrained environments.

More evidence is required in the form of larger or multicentred well-designed and adequately powered trials of fluoroquinolones in children, particularly in outpatient settings with adequate follow up and monitoring of adverse events.

To prevent inappropriate use of fluoroquinolones in children with prolonged fever, a step-wise approach to determining the cause of such fever in children and appropriate guidelines for management must be developed and evaluated in outpatient settings in areas endemic for enteric fever. Close monitoring of resistance patterns as well as check on indiscriminate use of alternate agents is needed.

Combination therapy may also reduce the rate of development of resistance in $S$. Typhi and $S$. Paratyphi, and could also be evaluated further in trial settings. Newer fluoroquinolones, such as gatifloxacin, may have efficacy against $\mathrm{NaR}$ strains; however more evidence is needed, including an investigation in tolerance and safety profile.

Trialists must improve both the methodological quality of randomized controlled trials and explicitly document the methods they use to minimize selection and observeration bias, including the use of double blinding.

Trialists should also standardize definitions of primary outcomes and the time points at which these are measured, including quantification of strains with reduced susceptibility to fluoroquinolones and other study drugs (including proportion of isolates with high MICs for fluoroquinolones, and proportion of MDR and $\mathrm{NaR}$ strains for each study arm).

\section{ACKNOWLEDGEMENTS}

Cochrane Infectious Diseases Group: Professor Paul Garner (Coordinating Editor) for guidance in the preparation of the protocol; Dr Vittoria Lutje (Information Retrieval Specialist) for help in searching databases and trial retrieval; and Dr Harriet MacLehose (Assistant Editor) for help during various stages of the protocol and review.

The first version of the review (Thaver 2005) was prepared (in part) during the Fellowship Programme organized by the Cochrane Infectious Disease Group in July 2003. The editorial base for the Cochrane Infectious Diseases Group is funded by the UK Department for International Development (DFID) for the benefit of developing countries. 


\section{R E F E R E N C E S}

\section{References to studies included in this review}

\section{Abejar 1993 \{published data only\}}

Abejar NH, Dimaano EM, Cabanban AB. Fleroxacin versus chloramphenicol in enteric fever. An open, randomized, parallel study. Philippine Journal of Internal Medicine 1993;31(6):327-30.

Alam 1995 \{published data only\}

Alam MN, Haq SA, Das KK, Baral PK, Mazid MN, Siddique RU, et al.Efficacy of ciprofloxacin in enteric fever: comparison of treatment duration in sensitive and multidrug resistant salmonella. American Journal of Tropical Medicine and Hygiene 1995;53(3): 306-11.

Arnold 1993 \{published data only\}

* Arnold K, Hong C, Nelwan R, Trujillo Z, Kadio A, Barros M, et al.Randomized comparative study of fleroxacin and chloramphenicol in typhoid fever. American Journal of Medicine 1993;94(3A):195S-200S.

Brown J, et al.Open label, randomized, parallel multicenter study comparing safety and efficacy of fleroxacin vs chloramphenicol. Roche Research 1990;November 1.

Bai 1995 \{published data only\}

Bai YM, Lin JB, Duan MT, Zhang Y, Liang B. Comparison of therapeutic effect of enoxacin and norfloxacin in the treatment of typhoid fever. Zhonghua Chuan Ran Bing Za Zhi [Chinese Journal of Infectious Diseases] 1995;13(2):117-8.

Bran 1991 \{published data only\}

Bran JL, Garcia JF, Mendez O. Comparative, double blind study of chloramphenicol and ciprofloxacin in the treatment of typhoid fever (abstract 751). Proceedings of the 31st Interscience Conference on Antimicrobial Agents and Chemotherapy; 1991 Sept 29-Oct 2; Chicago, Illinois. Washington, DC: American Society of Microbiology, 1991:224.

Cao 1999 \{published and unpublished data\} Cao XT, Kneen R, Nguyen TA, Truong DL, White NJ, Parry CM. A comparative study of ofloxacin and cefixime for treatment of typhoid fever in children. The Dong Nai Pediatric Center Typhoid Study Group. Pediatric Infectious Disease Journal 1999;18(3):245-8.

Chinh 2000 \{published and unpublished data\}

Chinh NT, Parry CM, Ly NT, Ha HD, Thong MX, Diep TS, et al.A randomized controlled comparison of azithromycin and ofloxacin for treatment of multidrug-resistant or nalidixic acidresistant enteric fever. Antimicrobial Agents and Chemotherapy 2000; 44(7):1855-9.

Cristiano 1995 \{published data only\}

Cirstiano P, Imparato L, Carpinelli C, Lauria F, Iovene MR Corrado MF, et al.Pefloxacin versus chloramphenicol in the therapy of typhoid fever. Infection 1995;23(2):103-6.

Dolecek 2008 \{published and unpublished data\}

Dolecek C, Phi La TT, Rang NN, Phuong LT, Vinh H, et al.A multi-center randomised controlled trial of gatifloxacin versus azithromycin for the treatment of uncomplicated typhoid fever in children and adults in Vietnam. PLoS ONE 2008;3(5):e2188 (111).
Duong 1995 \{published and unpublished data\} Duong NM, Chau NVV, Anh DCV, Hoa NTT, Tam DTH, Hai DT, et al.Short course fleroxacin in the treatment of typhoid fever. JAMA Southeast Asia 1995;11 Suppl:21-5.

Flores 1994 \{published data only\} Flores GR, Dorantes F, Aviles J. Safety and efficacy of oral ofloxacin vs oral ampicillin in the management of typhoid. Investigacion Medica Internacional 1994;21(2):88-92.

Gasem 2003 \{published data only\}

Gasem MH, Keuter M, Dolmans WM, Van Der Ven-Jongekriig J, Djokomoeljanto R, Van Der Meer JW. Persistence of Salmonellae in blood and bone marrow: randomized controlled trial comparing ciprofloxacin and chloramphenicol treatments against enteric fever. Antimicrobial Agents and Chemotherapy 2003;47(5):1727-31.

Girgis 1999 \{published and unpublished data\}

Girgis NI, Butler T, Frenck RW, Sultan Y, Brown FM, Tribble D, et al.Azithromycin versus ciprofloxacin for treatment of uncomplicated typhoid fever in a randomized trial in Egypt that included patients with multidrug resistance. Antimicrobial Agents and Chemotherapy 1999;43(6):1441-4.

Gottuzzo 1992 \{published data only\}

Gottuzzo E, Carillo C. Typhoid fever. Evaluation of the efficacy and safety of ciprofloxacin in comparison with chloramphenicol. In: HL Dupont editor(s). Use of quinolones in travel medicine. Second Conference on International Travel Medicine, Atlanta, Georgia 1991. Berlin, Germany: Springer-Verlag, 1992:16-22.

Hajji 1988 \{published and unpublished data\}

* Hajji M, el Mdaghri N, Benbachir M, el Filali KM, Himmich H. Prospective randomized comparative trial of pefloxacin versus cotrimoxazole in the treatment of typhoid fever in adults. European Journal of Clinical Microbiology and Infectious Diseases 1988;7(3): 361-3.

Himmich H. Comparison of activity and tolerance of pefloxacin and cotrimoxazole in the treatment of typhoid fever [Comparaison de l'activité et de la tolérance de la pefloxacine et du cotrimoxazole dans le traitement de la fievre typhoide]. Medecine et Maladies Infectieuses 1986;16:401.

Huai 2000 \{published data only\}

Huai Y, Zhu Q, Wang X. Ceftriaxone vs. norfloxacin in the treatment of resistant typhoid fever in 60 children. Zhonghua Er Ke Za Zhi [Chinese Journal of Pediatrics] 2000;38(6):386-8.

Jia 1994 \{published data only\}

* Jia FZ, Zhu JQ, Huang SY, Chen HK, Bai JY, Li ZC, et al.Clinical study on therapy of typhoid fever with pefloxacin. Zhonghua Chuan Ran Bing Za Zhi Zhonghua Chuan Ran Bing Za Zhi [Chinese Journal of Infectious Diseases] 1994;12 Suppl 3:134-6. Weng Y-L, Jia F-Z. Clinical efficacy evaluation of fluoroquinolones in typhoid fever (clinical analysis of 391 cases). Zhongguo Lin Chuang Yao Li Xue Za Zhi [Chinese Journal of Clinical Pharmacology] 1996;12(3):142-7.

Kalo 1997 \{published data only\}

Kalo T, Davachi F, Nushi A, Dedja S, Karapici L, Como N, et al.Therapeutic efficacy of perfloxacin in treatment of ampicillin- 
resistant typhoid fever in 7 days versus 10 days. International Journal of Infectious Diseases 1997;2(1):12-4.

Limson 1989 \{published data only\}

Limson BM, Littaua RT. Comparative study of ciprofloxacin versus co-trimoxazole in the treatment of Salmonella enteric fever. Infection 1989;17(2):105-6.

Morelli 1992 \{published data only\}

Morelli G, Mazzoli S, Tortoli E, Simonetti MT, Perruna F, Postiglione A. Fluoroquinolones versus chloramphenicol in the therapy of typhoid fever: a clinical and microbiological study. Current Therapeutic Research 1992;52(4):532-42.

Nalin 1987 \{published data only\}

Nalin DR, Hoagland VL, Acuna G, Bran JL, Carrilo C, Gotuzzo E, et al.Clinical trial of norfloxacin versus chloramphenicol therapy for acute typhoid fever. Progress in antimicrobial and anti-cancer chemotherapy. Proceedings of the 15th International Congress of Chemotherapy; 1987 July 19-24; Istanbul. Copenhagen, Munksgaard, 1987:1174-5.

Nguyen 1997 \{published and unpublished data\} Nguyen TC, Solomon T, Mai XT, Nguyen TL, Nguyen TT, Wain $\mathrm{J}$, et al.Short courses of ofloxacin for the treatment of enteric fever. Transactions of the Royal Society of Tropical Medicine and Hygiene 1997;91(3):347-9.

Pandit 2007 \{published and unpublished data\} Pandit, A, Arjyal, A, Day JN, Paudyal B, Dangol S, Zimmerman $\mathrm{MD}$, et al.An open randomized comparison of gatifloxacin versus cefixime for the treatment of uncomplicated enteric fever. PLoS ONE 2007;2(6):e542.

Parry 2007 \{published and unpublished data\}

Parry CM, Ho VA, Phuong IT, Bay PV, Lanh MN, Tung IT, et al.Randomized controlled comparison of ofloxacin, azithromycin, and an ofloxacin-azithromycin combination for treatment of multidrug-resistant and nalidixic acid-resistant typhoid fever. Antimicrobial Agents and Chemotherapy 2007;51(3):819-25.

Phongmany 2005 \{published and unpublished data\}

Phongmany S, Phetsouvanh R, Sisouphone S, Darasavath C, Vongphachane P, Rattanavong O, et al.A randomized comparison of oral chloramphenicol versus ofloxacin in the treatment of uncomplicated typhoid fever in Laos. Transactions of the Royal Society of Tropical Medicine and Hygiene 2005;99(6):451-8.

Quintero 1988 \{published data only\}

Quintero-Perez NP, Andrea-Villanueva J, Leon-Garnica M, BertinMontano M, Chagollan-Rodriguez E, Rodiguez-Noriega E. Comparative double blind efficacy and safety study of ciprofloxacin with chloramphenicol in the treatment of typhoid fever. Abstract number 385. Programs and abstracts of the 28th Interscience Conference on Antimicrobial Agents and Chemotherapy; 1988 Oct 23-26; Los Angeles, California. Washington, DC: American Society for Microbiology, 1988:177.

Sarma 1991 \{published and unpublished data\} Sarma PS, Durairaj P. Randomized treatment of patients with typhoid and paratyphoid fevers using norfloxacin or chloramphenicol. Transactions of the Royal Society of Tropical Medicine and Hygiene 1991;85(5):670-1.
Smith 1994 \{published and unpublished data\}

Smith MD, Duong NM, Hoa NT, Wain J, Ha HD, Diep TS, et al.Comparison of ofloxacin and ceftriaxone for short-course treatment of enteric fever. Antimicrobial Agents and Chemotherapy 1994;38(8):1716-20.

Tran 1994 \{published and unpublished data\}

Tran TH, Nguyen MD, Huynh DH, Nguyen TT, To SD, Le TP, et al.A randomized comparative study of fleroxacin and ceftriaxone in enteric fever. Transactions of the Royal Society of Tropical Medicine and Hygiene 1994;88(4):464-5.

Tran 1995 \{published and unpublished data\} Tran TH, Bethell DB, Nguyen TT, Wain J, To SD, Le TP, et al.Short course of ofloxacin for treatment of multidrug-resistant typhoid. Clinical Infectious Diseases 1995;20(4):917-23.

Unal 1996 \{published data only\}

Unal S, Hayran M, Tuncer S, Gur D, Uzun O, Akova M, et al.Treatment of enteric fever with pefloxacin for 7 days versus 5 days: a randomized clinical trial. Antimicrobial Agents and Chemotherapy 1996;40(12):2898-900.

Vinh 1996 \{published and unpublished data\}

Vinh H, Wain J, Vo TN, Cao NN, Mai TC, Bethell D, et al.Two or three days of ofloxacin treatment for uncomplicated multidrugresistant typhoid fever in children. Antimicrobial Agents and Chemotherapy 1996;40(4):958-61.

Vinh 2005 \{published and unpublished data\}

Vinh H, Duong NM, Phuong IT, Truong NT, Bay PV, Wain J, et al.Comparative trial of short-course ofloxacin for uncomplicated typhoid fever in Vietnamese children. Annals of Tropical Paediatrics 2005;25(1):17-22.

Wallace 1993 \{published data only\}

Wallace MR, Yousif AA, Mahroos GA, Mapes T, Threlfall EJ, Rowe $\mathrm{B}$, et al.Ciprofloxacin versus ceftriaxone in the treatment of multiresistant typhoid fever. European Journal of Clinical Microbiology and Infectious Diseases 1993;12(12):907-10.

Xiao 1991 \{published data only\}

Xiao YH, Wang QN, Du JZ, Zheng XP, Li ZZ, Qui J, et al.Clinical and laboratory study of the fluoroquinolone antibiotics in the treatment of multiresistant typhoid fever. Sichuan I Hseuh 1991;12 (4):196-8.

Yang 1991 \{published data only\} Yang JG, Wa XL, Shen XJ, Wang KL, Zhu XB, Chen ZY. Short course of ofloxacin in the treatment of 28 patients of typhoid fever. Xin Yao Yu Lin Chuang [New Drugs and Clinical Remedies] 1991;11 (2):79-80.

Yousaf 1992 \{published data only\}

Yousaf MH, Hasnain SS, Mohsin A, Ara N. A comparative study of efficacy and safety of three antimicrobials in the treatment of enteric fever. Pakistan Journal of Gastroenterology 1992;6(2):46-8.

Yu 1998 \{published data only\}

Yu Y-S, Zheng J-C, Gan M-J, Ma Y-L. Clinical study on the efficacy and safety of the levofloxacin used to treat the patients with typhoid fever or paratyphoid fever. Zhongguo Lin Chuang Yao Li Xue Za Zhi [Chinese Journal of Clinical Pharmacology] 1998;14(2):70-4.

References to studies excluded from this review 
Agalar 1997 \{published data only\}

Agalar C, Usubutun S, Tutuncu E, Turkyilmaz R. Comparison of two regimens for ciprofloxacin treatment of enteric infections. European Journal of Clinical Microbiology and Infectious Diseases 1997;16(11):803-6.

Akhtar 1989 \{published data only\} Akhtar MA, Karamat KA, Malik AZ, Hashmi A, Khan QM, Rasheed P. Efficacy of ofloxacin in typhoid fever, particularly in drug resistant cases. Reviews of Infectious Diseases 1989;2 Suppl 5:1193.

Akhtar 1992 \{published data only\} Akhtar MA, Hussain A, Karamat KA, Naqi N, Zubdi N. Role of ciprofloxacin in typhoid fever. Journal of Pakistan Medical Association 1992;42(1):9-10.

\section{Bavdekar 1991 \{published data only\}}

Bavdekar A, Chaudhari M, Bhave S, Pandit A. Ciprofloxacin in typhoid fever. Indian Journal Pediatrics 1991;58(3):335-9.

Bethell 1996 \{published data only\} Bethell DB, Day NP, Dung NM, McMullin C, Loan HT, Tam DT, et al.Pharmacokinetics of oral and intravenous ofloxacin in children with multidrug-resistant typhoid fever. Antimicrobial Agents and Chemotherapy 1996;40(9):2167-72.

Chakravorty 1991 \{published data only\} Chakravorty B, Jain N, Gupta B, Rajvanshi P, Sen MK, Krishna A. Chloramphenicol resistant enteric fever. Journal of Indian Medical Association 1993;91(1):10-13.

Chukwani 1998 \{published data only\} Chukwani CM, Onyemelukwe GC, Okonkwo PO, Coker HAB, Ifudu ND. Fleroxacin vs ciprofloxacin in the management of typhoid fever. Clinical Drugs Investigation 1998;16(4):279-88.

Daga 1994 \{published data only\}

Daga MK, Sarin K, Sarkar R. A study of culture positive multidrug resistant enteric fever--changing pattern and emerging resistance to ciprofloxacin. Journal of the Association of Physicians of India 1994; 42(8):599-600.

Hou 1993 \{published data only\}

Hou SR. Clinical study of Chinese ofloxacin. Zhonghua Yi Xue Za Zhi 1993;73(4):255-6.

Jinlong 1998 \{published data only\}

Jinlong Z, Wennan H, Guosheng M, Zhigang H, Jianping Z. Evaluation of effectiveness of ofloxacin and $S-(-)$ ofloxacin in treating typhoid fever. Zhonghua Chuan Ran Bing Za Zhi [Chinese Journal of Infectious Diseases] 1998;16(4):237.

Kumar 2007 \{published data only\}

Kumar R, Gupta N. Multidrug-resistant typhoid fever. Indian Journal of Pediatrics 2007;74(1):39-42.

Liberti 2000 \{published data only\}

Liberti A, Loiacono L. Ciprofloxacin versus chloramphenicol in the treatment of salmonella infection. International Journal of Antimicrobial Agents 2000;16(3):347-8.

Lu 1995 \{published data only\}

Lu Y, Zhang H, Mu G, Qiu D, Hu P. Clinical study of intravenous enoxacin versus cefotaxime in aute [sic] infectious diseases. Zhongguo Kang Sheng Su Za Zhi [Chinese Journal of Antibiotics] 1995;20(2):123-8.
Nelwan 1995 \{published data only\}

Nelwan RH, Hendarwanto, Zulkarnain I, Gunawan J,

Supandiman I, Yusuf H, et al.A comparative study of short course ciprofloxacin treatment in typhoid and paratyphoid fever. Drugs 1995;49 Suppl 2:463-5.

Peyramond 1986 \{published data only\}

Peyramond D, Tiguad S, Lucht F, Vedrinne JM, Salord F, Bertrand JL. Clinical evaluation of ofloxacin in the treatment of various infections [Bilan d'utilisation de l'ofloxacine dans le traitement d'infections variées]. Pathologie-Biologie 1986;34(5):471-5.

Secmeer 1997 \{published data only\}

Secmeer G, Kanra G, Figen G, Akan O, Ceyhan M, Ecevit Z. Ofloxacin versus co-trimoxazole in the treatment of typhoid fever in children. Acta Paediatrica Japonica 1997;39(2):218-21.

Singh 1993 \{published data only\} Singh CP, Singh N, Brar GK, Lal G, Kumar H. Efficacy of ciprofloxacin and norfloxacin in multidrug resistant enteric fever in adults. Journal of the Indian Medical Association 1993;91(6):156-7.

Suhendro 2007 \{published data only\}

Suhendro, Chen K, Pohan HT. Open study on efficacy and tolerability of ciprofloxacin XR compared with ciprofloxacin BID in the treatment of typhoid fever. Acta Medica Indonesiana 2007;39 (1):22-6.

Takkar 1994 \{published data only\}

Takkar VP, Kumar R, Khurana S, Takkar R. Comparison of ciprofloxacin versus cephelexin and gentamicin in the treatment of multi-drug resistant typhoid fever. Indian Pediatrics 1994;31(2): 200-1.

Tanphaichitra 1986 \{published data only\}

Tanphaichitra D, Sahaphong S, Srimuang S. Ofloxacin, a new quinolone in the treatment of genitourinary and enteric infections. Infection 1986;14 Suppl 4:321-3.

Uwaydah 1992 \{published data only\} Uwaydah AK, al Soub H, Matar I. Randomized prospective study comparing two dosage regimens of ciprofloxacin for the treatment of typhoid fever. Journal of Antimicrobial Chemotherapy 1992;30 (5):707-11.

Wain 1997 \{published data only\}

Wain J, Hoa NTT, Chinh NT, Vinh H, Everett J, Diep TS, et al.Quinolone-resistant Salmonella typhi in Viet Nam: molecular basis of resistance and clinical response to treatment. Clinical Infectious Diseases 1997;25(6):1404-10.

Zavala 1989 \{published data only\} Zavala-Trujillo I, Nava-Zavala A, Marcano MG, Renteria M. Fleroxacin in the treatment of enteric fever and salmonellosis in adults. Reviews of Infectious Diseases 1989;2 Suppl 5:1188-9.

Zhang 1991 \{published data only\} Zhang YY, Zhun BY, Jiang SC. Clinical evaluation of enoxacin. Zhonghua Nei Ke Za Zhi [Chinese Journal of Internal Medicine] 1991;30(8):480-3, 521.

ZhongYang 1997 \{published data only\} ZhongYang H, Zheng L, Xinchung L, Hanjun H, Yi L X. Compared observation on therapeutic effect of ofloxacin and norfloxacin in treatment of typhoid fever. Zhonghua Chuan Ran 
Bing Za Zhi [Chinese Journal of Infectious Diseases] 1997;15(4): 229-30.

\section{References to studies awaiting assessment}

Flores 1991 \{published data only\}

Flores GR. Pharmacological treatment of typhoid fever: A comparative study of ciprofloxacin versus trimethoprimsulfamethoxazole [Tratamiento farmacológico del paciente con fiebre tifoidea: estudio comparativo entre ciprofloxacina y trimetoprim-sulfametoxazol]. Investigacion Medica International 1991;17(4):185-8.

Soewandojo 1992 \{published data only\}

Soewandojo E, Suharto JR. Comparative study of ciprofloxacin vs chloramphenicol in patients with typhoid fever. Typhoid fever, profile, diagnosis and treatment in 1990. Proceedings of the 1st ISAC International Symposium. Jakarta: Acta Medica Indonesia, 1992:119-25.

\section{References to ongoing studies}

\section{ISRCTN53258327 \{unpublished data only\}}

ISRCTN53258327. An open randomised study to assess the efficacy of gatifloxacin versus chloramphenicol for the treatment of uncomplicated typhoid fever in Kathmandu, Nepal. www.controlled-trials.com/ISRCTN53258327 (accessed 26 November 2007).

ISRCTN66534807 \{unpublished data only\}

ISRCTN66534807. A randomised clinical trial of Azithromycin versus Ofloxacin in the treatment of adults with uncomplicated typhoid fever at Mahosot Hospital, Vientiane, Lao People's Democratic Republic (PDR). www.controlled-trials.com/ ISRCTN66534807.

\section{Additional references}

\section{Acharya 1995}

Acharya G, Butler T, Ho M, Sharma PR, Tiwari M, Adhikari RK, et al.Treatment of typhoid fever: randomized trial of a three-day course of ceftriaxone versus a fourteen-day course of chloramphenicol. American Journal of Tropical Medicine and Hygiene 1995;52(2):162-5.

Adachi 2005

Adachi T, Sagara H, Hirose K, Watanabe H. Fluoroquinoloneresistant Salmonella paratyphi A. Emerging Infectious Diseases 2005; 11(1):172-4.

Ahmad 2002

Ahmad K. Experts call for surveillance of drug-resistant typhoid at a global level. Lancet 2002;359(9306):592.

\section{Ahmed 2006}

Ahmed D, D'Costa LT, Alam K, Nair GB, Hossain MA. Multidrug-resistant Salmonella enterica serovar typhi isolates with high-level resistance to ciprofloxacin in Dhaka, Bangladesh. Antimicrobial Agents and Chemotherapy 2006;50(10):3516-7.

Asna 2003

Asna SM, Haq JA, Rahman MM. Nalidixic acid-resistant Salmonella enterica serovar typhi with decreased susceptibility to ciprofloxacin caused treatment failure: a report from Bangladesh. Japan Journal of Infectious Diseases 2003;56(1):32-3.

Bhutta 1994

Bhutta ZA, Khan IA, Molla AM. Therapy of multidrug-resistant typhoid fever with oral cefixime vs. intravenous ceftriaxone.

Pediatric Infectious Diseases Journal 1994;13(11):990-4.

Bhutta 1996

Bhutta ZA. Impact of age and drug resistant on mortality in typhoid fever. Archives of Disease in Childhood 1996;75(3):214-7.

Biswal 1994

Biswal N, Mathai B, Bhatia BD, Srinivasan S. Use of ciprofloxacin and its antimicrobial resistance in typhoid fever. Indian Pediatrics 1994;31(2):229-30.

\section{Brooks 2005}

Brooks WA, Hossain A, Goswami D, Nahar K, Alam K, Ahmed N, et al.Bacteremic typhoid fever in children in an urban slum, Bangladesh. Emerging Infectious Diseases 2005;11(2):326-9.

\section{Brown 1994}

Brown NM, Millar MR, Frost JA, Rowe B. Ciprofloxacin resistance in Salmonella paratyphi A. Journal of Antimicrobial Agents and Chemotherapy 1994;33(6):1258-9.

\section{Butler 1991}

Butler T, Islam A, Kabir I, Jones PK. Patterns of morbidity and mortality in typhoid fever dependent on age and gender: review of 552 hospitalized patients with diarrhea. Reviews of Infectious Diseases 1991;13(1):85-90.

Butt 2003

Butt T, Ahmad RN, Mahmood A, Zaidi S. Ciprofloxacin treatment failure in typhoid fever case, Pakistan. Emerging Infectious Diseases 2003;9(12):1621-2.

\section{Butt 2005}

Butt T, Ahmad RN, Salman M, Kazmi SY. Changing trends in drug resistance among typhoid salmonellae in Rawalpindi, Pakistan. Eastern Mediterranean Health Journal 2005;11(5-6):1038-44.

\section{Chandel 2000}

Chandel DS, Chaudhry R, Dhawan B, Pandey A, Dey AB. Drugresistant Salmonella enterica serotype paratyphi A in India. Emerging Infectious Diseases 2000;6(4):420-1.

\section{Chandel 2001}

Chandel DS, Chaudhry R. Enteric fever treatment failures: a global concern. Emerging Infectious Diseases 2001;7(4):762-3.

Chau 2007

Chau TT, Campbell JI, Galindo CM, Van Minh HN, Diep TS, Nga TT, et al.Antimicrobial drug resistance of Salmonella enterica serovar typhi in Asia and molecular mechanism of reduced susceptibility to the fluoroquinolones. Antimicrobial Agents and Chemotherapy 2007;51(12):4315-23.

Cleary 2000

Cleary TG. Salmonella. Nelson textbook of pediatrics. 16th Edition. Philadelphia: Saunders, 2000:842-8.

\section{CLSI 2006}

Clinical and Laboratory Standards Institute (CLSI). Performance standards for antimicrobial susceptibility testing; Sixteenth 
informational supplement. Vol. 26(3), Clinical and Laboratory Standards Institute: Wayne, PA, 2006:109.

\section{Committee 2006}

Committee on Infectious Diseases. The use of systemic fluoroquinolones. Pediatrics 2006;118(3):1287-92.

\section{Congeni 2002}

Congeni BL, Thomson RB Jr. Fluoroqinolones: consideration for future use. Pediatric Infectious Diseases Journal 2002;21(4):345-6.

\section{Cooke 2006}

Cooke FJ, Wain J, Threlfall EJ. Fluoroquinolone resistance in Salmonella typhi. BMJ 2006;333(7563):353-4.

\section{Cross 2001}

Cross JT Jr. New anti-infectives. Fluoroquinolones. Seminars in Pediatric Infectious Diseases. Vol. 12, W.B. Saunders Company, 2001:211-23.

\section{Crump 2004}

Crump JA, Luby SP, Mintz ED. The global burden of typhoid fever Bulletin of the World Health Organization 2004;82(5):346-53.

\section{Davies 1998}

Davies HTO, Crombie IK. What is a systematic review?. What is...? series 2001;1(5):1-6.

Dimitrov 2007

Dimitrov T, Udo EE, Albaksami O, Kilani AA, Shehab el-DM. Ciprofloxacin treatment failure in a case of typhoid fever caused by Salmonella enterica serotype Paratyphi A with reduced susceptibility to ciprofloxacin. Journal of Medical Microbiology 2007;56(Pt 2):277-9.

\section{Fish 2001}

Fish DN. Fluoroquinolone adverse effects and drug interactions. Pharmacotherapy 2001;21(10 Part 2):253-72.

\section{Gendrel 2003}

Gendrel D, Chalumeau M, Moulin F, Raymond J.

Fluoroquinolones in paediatrics: a risk for the patient or for the community?. Lancet Infectious Diseases 2003;3(9):537-46.

\section{Harish 2004}

Harish BN, Madhulika U, Parija SC. Isolated high-level ciprofloxacin resistance in Salmonella enterica subsp. enterica serotype Paratyphi A. Journal of Medical Microbiology 2004;53(Pt 8):819.

\section{Higgins 2006}

Higgins J, Green S, editors. Highly sensitive search strategies for identifying reports of randomized controlled trials in MEDLINE. Cochrane Handbook for Systematic Reviews of Interventions 4.2.6 [updated September 2006]; Appendix 5b. www.cochrane.org/ resources/handbook/hbook.htm (accessed 26 November 2007).

\section{Hooper 2000}

Hooper DC. Quinolones. In: Mandell GL, Bennett JE, Dolin R editor(s). Mandell, Douglas and Bennett's principles and practice of infectious diseases. 5th Edition. Philadelphia. London: Churchill Livingstone, 2000:405-20.

\section{Islam 1988}

Islam A, Butler T, Nath SK, Alam NH, Stoeckel K, Houser HB, et al.Randomized treatment of patients with typhoid fever by using ceftriaxone or chloramphenicol. Journal of Infectious Diseases 1988; 158(4):742-7.

\section{Ivanoff 1995}

Ivanoff B. Typhoid fever: global situation and WHO recommendations. Southeast Asian Journal of Topical Medicine and Public Health 1995;26 Suppl 2:1-6.

\section{Jesudason 2005}

Jesudason MV. Paratyphoid fever in Vellore, South India. Tropical Doctor 2005;35(3):191.

Joshi 2007

Joshi S, Amarnath SK. Fluoroquinolone resistance in Salmonella typhi and S. paratyphi A in Bangalore, India. Transactions of the Royal Society of Tropical Medicine and Hygiene 2007;101(3):308-10.

\section{Jüni 2001}

Jüni P, Altman DG, Egger M. Systematic reviews in health care: Assessing the quality of controlled clinical trials. BMJ 2001;323 (7303):42-6.

\section{Karunanayake 2004}

Karunanayake L, Atukorala SD. Enteric fever caused by Salmonella isolates with decreased susceptibility to ciprofloxacin. Ceylon Medical Journal 2004;49(4):137-8.

\section{Lasserre 1991}

Lasserre R, Sangalang RP, Santiago L. Three-day treatment of typhoid fever with two different doses of ceftriaxone, compared to 14-day therapy with chloramphenicol: a randomized trial. Journal of Antimicrobial Agents and Chemotherapy 1991;28(5):765-72.

\section{Lee 2000}

Lee TP, Hoffman SL. Typhoid fever. In: Strickland GT editor(s). Hunter's Tropical medicine and emerging infectious diseases. 8th Edition. Philadelphia. London: Saunders, 2000:471-83.

Lin 2000

Lin FY, Vo AH, Phan VB, Nguyen TT, Bryla D, Tran CT, et al.The epidemiology of typhoid fever in the Dong Thap Province, Mekong Delta region of Vietnam. American Journal of Tropical Medicine and Hygiene 2000;62(5):644-8.

\section{Mahle 1993}

Mahle WT, Levine MM. Salmonella typhi infection in children younger than five years of age. Pediatric Infectious Diseases Journal 1993;12(8):627-31.

\section{Mahmood 2000}

Mahmood A. Multidrug resistant Salmonella paratyphi A. Journal of College of Physicians and Surgeons of Pakistan 2000;10(11):444.

\section{Manchanda 2006}

Manchanda V, Bhalla P, Sethi M, Sharma VK. Treatment of enteric fever in children on the basis of current trends of antimicrobial susceptibility of Salmonella enterica serovar typhi and paratyphi A. Indian Journal of Medical Microbiology 2006;24(2):101-6.

\section{Maskey 2006}

Maskey AP, Day JN, Phung QT, Thwaites GE, Campbell JI, Zimmerman M, et al.Salmonella enterica serovar Paratyphi A and S. enterica serovar Typhi cause indistinguishable clinical syndromes in Kathmandu, Nepal. Clinical Infectious Diseases 2006;42(9): 1247-53.

\section{Maskey 2008}

Maskey AP, Basnyat B, Thwaites GE, Campbell JI, Farrar JJ, Zimmerman MD. Emerging trends in enteric fever in Nepal: 9124 
cases confirmed by blood culture 1993-2003. Transactions of the Royal Society of Tropical Medicine and Hygiene 2008;102:91-5.

\section{Mermin 1998}

Mermin JH, Townes JM, Gerber M, Dolan N, Mintz ED, Tauxe RV. Typhoid fever in the United States, 1985-1994: changing risks of international travel and increasing antimicrobial resistance. Archives of Internal Medicine 1998;158(6):633-8.

\section{Miller 2000}

Miller SI, Pegues DA. Salmonella species, including Salmonella typhi. In: Mandell GL, Bennett JE, Dolin R editor(s). Mandell, Douglas and Bennett's principles and practice of infectious diseases. 5 th Edition. Philadelphia. London: Churchill Livingstone, 2000: $2344-56$.

\section{Mohanty 2006}

Mohanty S, Renuka K, Sood S, Das BK, Kapil A. Antibiogram pattern and seasonality of Salmonella serotypes in a North Indian tertiary care hospital. Epidemiology and infection 2006;134(5): 961-6.

\section{Murdoch 1998}

Murdoch DA, Banatvaia N, Bone A, Shoismatulloev BI, Ward LR, Threlfall EJ, et al.Epidemic ciprofloxacin-resistant Salmonella typhi in Tajikistan. Lancet 1998;351(9099):339.

\section{Ochiai 2005}

Ochiai RL, Wang X, von SL, Yang J, Bhutta ZA, Bhattacharya SK, et al.Salmonella paratyphi A rates, Asia. Emerging Infectious Diseases 2005;11(11):1764-6.

Parry 2002

Parry CM, Hien TT, Dougan G, Whilte NJ, Farrar JJ. Typhoid fever. New England Journal of Medicine 2002;347(22):1770-82.

\section{Parry 2004}

Parry CM. The treatment of multidrug-resistant and nalidixic acidresistant typhoid fever in Viet Nam. Transactions of the Royal Society of Tropical Medicine and Hygiene 2004;98(7):413-22.

\section{Renuka 2005}

Renuka K, Sood S, Das BK, Kapil A. High-level ciprofloxacin resistance in Salmonella enterica serotype Typhi in India. Journal of Medical Microbiology 2005;54(Pt 10):999-1000.

Review Manager 4.2.

The Nordic Cochrane Centre, The Cochrane Collaboration. Review Manager (RevMan). 4.2 for Windows. Copenhagen: The Nordic Cochrane Centre, The Cochrane Collaboration, 2002.

Richens 1995

Richens J. Typhoid in the highlands of Papua New Guinea 19841990: a hospital-based perspective. Papua and New Guinea Medical Journal 1995;38(4):305-14.

Richens 2000

Richens J. Typhoid fever. In: Armstrong D, Cohen J editor(s). Infectious diseases. 1st Edition. London: Mosby, 2000:6-24.1-4.

Rodrigues 2003

Rodrigues C, Shenai S, Mehta A. Enteric fever in Mumbai, India: the good news and the bad news. Clinical Infectious Diseases 2003; 36(4):535.

Rowe 1995

Rowe B, Ward LR, Threlfall EJ. Ciprofloxacin-resistant Salmonella typhi in the UK. Lancet 1995;346(8985):1302.

\section{Rupali 2004}

Rupali P, Abraham OC, Jesudason MV, John TJ, Zachariah A, Sivaram $S$, et al.Treatment failure in typhoid fever with ciprofloxacin susceptible Salmonella enterica serotype Typhi. Diagnostic Microbiology and Infectious Disease 2004;49(1):1-3.

Saha 2001

Saha SK, Baqui AH, Hanif M, Darmstadt GL, Ruhulamin M, Nagatake T, et al.Typhoid fever in Bangladesh: implications for vaccination policy. Pediatric Infectious Diseases Journal 2001;20(5): $521-4$.

Saha 2003

Saha MR, Dutta P, Palit A, Dutta D, Bhattacharya MK, Mitra U, et al.A note on incidence of typhoid fever in diverse age groups in Kolkata, India. Japan Journal of Infectious Diseases 2003;56(3): 121-2.

\section{Siddiqui 2006}

Siddiqui FJ, Rabbani F, Hasan R, Nizami SQ, Bhutta ZA. Typhoid fever in children: some epidemiological considerations from Karachi, Pakistan. International Journal of Infectious Diseases 2006; 10(3):215-22.

\section{Simonin 1999}

Simonin MA, Gegout-Pottie P, Minn A, Gillet P, Netter P, Terlain P. Proteoglycan and collagen biochemical variations during fluoroquinolone-induced chondrotoxicity in mice. Antimicrobial Agents and Chemotherapy 1999;43(12):2915-21.

Sinha 1999

Sinha A, Sazawal S, Kumar R, Sood S, Reddaiah VP, Singh B, et al.Typhoid fever in children aged less than 5 years. Lancet 1999; 354(9180):734-7.

\section{Slinger 2004}

Slinger R, Desjardins M, McCarthy AE, Ramotar K, Jessamine P, Guibord C, et al.Suboptimal clinical response to ciprofloxacin in patients with enteric fever due to Salmonella spp. with reduced fluoroquinolone susceptibility: a case series. BMC Infectious Diseases 2004;4:36.

Sood 1999

Sood S, Kapil A, Das B, Jain Y, Kabra SK. Re-emergence of chloramphenicol-sensitive Salmonella typhi. Lancet 1999;353 (9160):1241-2.

Stern 1997

Stern JM, Simes RJ. Publication bias: evidence of delayed publication in a cohort study of clinical research projects. BMJ 1997;315(7109):640-5.

Sur 2006

Sur D, von SL, Manna B, Dutta S, Deb AK, Sarkar BL, et al.The malaria and typhoid fever burden in the slums of Kolkata, India: data from a prospective community-based study. Transactions of the Royal Society of Tropical Medicine and Hygiene 2006;100(8):725-33.

Takkar 1995

Takkar VP, Kumar R, Takkar R, Khurana S. Resurgence of chloramphenicol sensitive Salmonella typhi. Indian Pediatrics 1995 32(5):586-7.

Threlfall 2001

Threlfall EJ, Ward LR. Decreased susceptibility to ciprofloxacin in Salmonella enterica serotype typhi, United Kingdom. Emerging Infectious Diseases 2001;7(3):448-50. 


\section{Threlfall 2003}

Threlfall EJ, Fisher IS, Berghold C, Gerner-Smidt P, Tschape H, Cormican $\mathrm{M}$, et al.Trends in antimicrobial drug resistance in Salmonella enterica serotypes Typhi and Paratyphi A isolated in Europe, 1999-2001. International Journal of Antimicrobial Agents 2003;22(5):487-91.

Turner 2006

Turner AK, Nair S, Wain J. The acquisition of full fluoroquinolone resistance in Salmonella Typhi by accumulation of point mutations in the topoisomerase targets. Journal of Antimicrobial Chemotherapy 2006;58(4):733-40.

Walia 2005

Walia M, Gaind R, Mehta R, Paul P, Aggarwal P, Kalaivani M. Current perspectives of enteric fever: a hospital-based study from India. Annals of Tropical Paediatrics 2005;25(3):161-74.

\section{Walia 2006}

Walia M, Gaind R, Paul P, Mehta R, Aggarwal P, Kalaivani M. Agerelated clinical and microbiological characteristics of enteric fever in India. Transactions of the Royal Society of Tropical Medicine and Hygiene 2006;100(10):942-8.

\section{Wasfy 2002}

Wasfy MO, Frenck R, Ismail TF, Masour H, Malone JL, Mahoney
FJ. Trends of multiple-drug resistance among Salmonella serotype Typhi isolates during a 14-year period in Egypt. Clinical Infectious Diseases 2002;35(10):1265-8.

\section{WHO 2003}

World Health Organization. Dept. of Vaccines and Biologicals. Treatment of typhoid fever. Background document: the diagnosis, treatment and prevention of typhoid fever [WHO/V\&B/03.07]. Geneva: World Health Organization, 2003:19-23.

\section{Woods 2006}

Woods CW, Murdoch DR, Zimmerman MD, Glover WA, Basnyat $B$, Wolf $L$, et al.Emergence of Salmonella enterica serotype Paratyphi A as a major cause of enteric fever in Kathmandu, Nepal. Transactions of the Royal Society of Tropical Medicine and Hygiene 2006;100(11):1063-7.

\section{References to other published versions of this review}

\section{Thaver 2005}

Thaver D, Zaidi AK, Critchley J, Madni SA, Bhutta ZA. Fluoroquinolones for treating typhoid and paratyphoid fever (enteric fever). Cochrane Database of Systematic Reviews 2005, Issue 2. [DOI: 10.1002/14651858.CD004530.pub2]

* Indicates the major publication for the study 


\section{CHARACTERISTICS OF STUDIES}

\section{Characteristics of included studies [ordered by study ID]}

\section{Abejar 1993}

\begin{tabular}{l|l} 
Methods & $\begin{array}{l}\text { Generation of allocation sequence: unclear } \\
\text { Allocation concealment: unclear } \\
\text { Blinding: open } \\
\text { Inclusion of all randomized culture-positive participants in the final analysis: } 100 \%\end{array}$ \\
\hline Participants & $\begin{array}{l}30 \text { analysed }^{a}: 15 \text { in fleroxacin group; } 15 \text { in chloramphenicol group } \\
\text { Adult inpatients } \\
\text { Inclusion criteria: clinical with blood culture positive } \\
\text { Exclusion criteria: children and culture negative }\end{array}$ \\
\hline Interventions & $\begin{array}{l}\text { 1. Fleroxacin ( } 400 \text { mg oral once daily for } 10 \text { days) } \\
\text { 2. Chloramphenicol (50 mg/kg/day in } 3 \text { divided doses every } 8 \text { hours for } 14 \text { days) }\end{array}$ \\
\hline Outcomes & $\begin{array}{l}\text { 1. Clinical failure } \\
\text { 2. Microbiological failure } \\
\text { 3. Relapse } \\
\text { 4. Fever clearance time (no SD) } \\
\text { 5. Other adverse events }\end{array}$ \\
\hline Notes & $\begin{array}{l}\text { Location: Philippines } \\
\text { Date: not reported } \\
\text { Severity of illness at entry: not reported }\end{array}$ \\
\hline
\end{tabular}

\section{Alam 1995}

Methods Generation of allocation sequence: unclear

Allocation concealment: unclear

Blinding: open

Inclusion of all randomized culture-positive participants in the final analysis: 64/72 (88.9\%)

Participants 69 analysed $^{a}: 35$ in 10-day group: 34 in 14-day group

Adults (18 to 65 years) and 11 children (< 18 years)

Both outpatients and inpatients (ciprofloxacin 10-day group had 20 outpatients and 14 inpatients, ciprofloxacin 14day group had 21 outpatients and 14 inpatients)

Inclusion criteria: blood or bone marrow culture positive for $S$. Typhi or $S$. Paratyphi

Exclusion criteria: hypersensitivity to quinolones; severe renal disease; pregnant or lactating; patients < 18 years were randomized only if had MDR strain

Interventions 1. Ciprofloxacin (500 mg oral twice daily for 10 days)

2. Ciprofloxacin (500 mg oral twice daily for 14 days)

Outcomes 1. Clinical failure

2. Microbiological failure

3. Relapse

Fluoroquinolones for treating typhoid and paratyphoid fever (enteric fever) (Review)

Copyright ( 2008 The Cochrane Collaboration. Published by John Wiley \& Sons, Ltd. 
Alam 1995 (Continued)

4. Fever clearance time

5. Convalescent faecal carriage

6. Serious adverse events

7. Other adverse events

Notes Location: Bangladesh

Date: $1992-3$

Severity of illness at entry: not reported

\section{Arnold 1993}

Methods Generation of allocation sequence: unclear

Allocation concealment: unclear

Blinding: open

Inclusion of all randomized culture-positive participants in the final analysis: $100 \%$

Participants 91 analysed $^{a}: 24$ in the fluoroquinolone 7-day group; 33 in the fluoroquinolone 14-day group; 34 in the chloramphenicol group

Adult inpatients aged 18 to 65 years

Inclusion criteria: clinical

Exclusion criteria: fever > 14 days; signs of typhoid fever > 21 days before enrolment; pregnant or lactating; hypersensitivity to chloramphenicol; nalidixic acid and its derivatives; history of cerebral disorders; severe concomitant disease; concomitant antimicrobial treatment

Interventions 1 . Fleroxacin (400 mg oral once daily for 7 days)

2. Fleroxacin ( $400 \mathrm{mg}$ oral once daily for 14 days)

3. Chloramphenicol $(50 \mathrm{mg} / \mathrm{kg} /$ day oral for 14 days $)$

Outcomes 1. Clinical failure

2. Microbiological failure

3. Relapse

4. Fever clearance time (time to event)

5. Convalescent faecal carriage

Notes Location: multicentre; country names not reported, but authors' affiliations were Brazil, Mexico, Korea, Indonesia, and Ivory Coast

Date: not available

Severity of illness at entry: major complications were excluded

Bai 1995

Methods Generation of allocation sequence: unclear

Allocation concealment: unclear

Blinding: open

Inclusion of all randomized culture-positive participants in the final analysis: $100 \%$

Fluoroquinolones for treating typhoid and paratyphoid fever (enteric fever) (Review)

Copyright @ 2008 The Cochrane Collaboration. Published by John Wiley \& Sons, Ltd. 
Bai 1995 (Continued)

\begin{tabular}{ll}
\hline Participants & $\begin{array}{l}102 \text { analysed }^{a}: 52 \text { in enoxacin group; } 50 \text { in norfloxacin group } \\
\text { Adult inpatients aged } 17 \text { to } 64 \text { years } \\
\text { Inclusion criteria: clinical with blood or bone marrow culture positive for } S . \text { Typhi } \\
\text { Exclusion criteria: not mentioned }\end{array}$ \\
\hline Interventions & $\begin{array}{l}\text { 1. Enoxacin (300 mg oral twice daily for } 10 \text { days or } 3 \text { to } 5 \text { days after afebrile) } \\
\text { 2. Norfloxacin (200 mg oral } 3 \text { to } 4 \text { times a day for } 14 \text { days or } 3 \text { to } 5 \text { days after afebrile) }\end{array}$ \\
\hline Outcomes & $\begin{array}{l}\text { 1. Clinical failure } \\
\text { 2. Fever clearance time } \\
\text { 3. Complications } \\
\text { 4. Serious adverse events } \\
\text { 5. Other adverse events }\end{array}$ \\
\hline Notes & $\begin{array}{l}\text { Location: China (Chinese language) } \\
\text { Date: } 1989-94 \\
\text { Severity of illness at entry: not reported }\end{array}$ \\
\hline
\end{tabular}

Bran 1991

\begin{tabular}{ll}
\hline Methods & $\begin{array}{l}\text { Generation of allocation sequence: unclear } \\
\text { Allocation concealment: unclear } \\
\text { Blinding: double } \\
\text { Inclusion of all randomized culture-positive participants in the final analysis: } 100 \%\end{array}$ \\
\hline Participants & $\begin{array}{l}102 \text { analysed } \text { : } 51 \text { in ciprofloxacin group; } 51 \text { in chloramphenicol group; only the total number of participants (102) } \\
\text { was provided, but we assumed } 51 \text { in each group } \\
\text { Age not mentioned (adult dosages used); most probably inpatients } \\
\text { Inclusion criteria: blood and/or bone marrow culture positive for } S . \text { Typhi } \\
\text { Exclusion criteria: not reported }\end{array}$ \\
\hline Interventions & $\begin{array}{l}\text { 1. Ciprofloxacin (500 mg oral twice daily for } 10 \text { days) } \\
\text { 2. Chloramphenicol (750 mg oral every } 6 \text { hours for } 14 \text { days) }\end{array}$ \\
\hline Outcomes & $\begin{array}{l}\text { 1. Microbiological failure } \\
\text { 2. Fever clearance time (no SD) } \\
\text { 3. Convalescent faecal carriage } \\
\text { 4. Serious adverse events } \\
\text { 5. Other adverse events }\end{array}$ \\
\hline Notes & $\begin{array}{l}\text { Location: Guatemala } \\
\text { Date: not reported } \\
\text { Severity of illness at entry: not reported } \\
\text { Conference abstract }\end{array}$ \\
\hline
\end{tabular}


Methods Generation of allocation sequence: computer-generated

Allocation concealment: sealed envelopes

Blinding: open

Inclusion of all randomized culture-positive participants in the final analysis: 40/82 (49\%)

Participants 82 analysed $^{a}: 38$ in ofloxacin group; 44 in cefixime group

Children inpatients aged $<15$ years

Inclusion criteria: fever and no obvious source of infection for $>7$ days or $<7$ days if family history of typhoid fever Exclusion criteria: severe disease; hypersensitivity to quinolones or third-generation cephalosporins; received either drug during this illness; or responded to ampicillin, chloramphenicol, or co-trimoxazole

Interventions 1 . Ofloxacin $(10 \mathrm{mg} / \mathrm{kg} /$ day oral in 2 divided doses for 5 days $)$

2. Cefixime $(20 \mathrm{mg} / \mathrm{kg} /$ day oral in 2 divided doses for 7 days)

\begin{tabular}{l|l} 
Outcomes & 1. Clinical failure \\
& 2. Microbiological failure \\
3. Relapse \\
4. Fever clearance time \\
5. Complications \\
6. Length of hospitalization \\
7. Convalescent faecal carriage \\
8. Serious adverse events \\
9. Other adverse events
\end{tabular}

Notes Location: Vietnam

Date: $1995-6$

Severity of illness at entry: all uncomplicated

Author provided further information

\section{Chinh 2000}

Methods Generation of allocation sequence: computer-generated

Allocation concealment: sealed envelopes

Blinding: open

Inclusion of all randomized culture-positive participants in the final analysis: 38/91(42\%)

Participants 88 analysed $^{a}: 44$ in ofloxacin group; 44 in azithromycin group

Adult inpatients aged $\geq 15$ years

Inclusion criteria: clinical with blood culture positive for $S$. Typhi or $S$. Paratyphi

Exclusion criteria: severe or complicated disease; significant underlying disease; hypersensitivity to either trial drug; pregnant; history of treatment with fluoroquinolone or third-generation cephalosporins or macrolides within 1 week of admission

Interventions 1 . Ofloxacin (200 mg oral twice daily for 5 days at $8 \mathrm{mg} / \mathrm{kg} / \mathrm{day}$ )

2. Azithromycin ( $1 \mathrm{~g}$ oral daily for 5 days at $20 \mathrm{mg} / \mathrm{kg} /$ day)

Outcomes 1. Clinical failure

2. Microbiological failure

3. Relapse

Fluoroquinolones for treating typhoid and paratyphoid fever (enteric fever) (Review)

Copyright () 2008 The Cochrane Collaboration. Published by John Wiley \& Sons, Ltd. 


\section{Chinh 2000 (Continued)}

4. Fever clearance time (mean and $95 \%$ confidence intervals; SD calculated by review author)

5. Complications

6. Length of hospitalization (mean and 95\% confidence interval; SD calculated by review author)

7. Convalescent faecal carriage

8. Serious adverse events

9. Other adverse events (number of events stated)

$\begin{array}{ll}\text { Notes } & \text { Location: Vietnam } \\ & \text { Date: not available } \\ \text { Severity of illness at entry: all uncomplicated } \\ \text { Author provided further information }\end{array}$

\section{Cristiano 1995}

Methods Generation of allocation sequence: computer-generated

Allocation concealment: unclear

Blinding: open

Inclusion of all randomized culture-positive participants in the final analysis: $100 \%$

Participants 60 analysed $^{a}: 30$ in pefloxacin group; 30 in chloramphenicol group

Adult inpatients aged 17 to 64 years

Inclusion criteria: severe culture-positive typhoid sepsis

Exclusion criteria: received drug active against $S$. Typhi

Interventions 1. Pefloxacin (1200 mg intravenous in 3 divided doses every 8 hours for 5 days, then oral for 10 days)

2. Chloramphenicol ( $2 \mathrm{~g}$ oral in 4 divided doses every 6 hours for 15 days)

$\begin{array}{ll}\text { Outcomes } & \text { 1. Clinical failure } \\ & \text { 2. Microbiological failure } \\ \text { 3. Relapse } \\ \text { 4. Fever clearance time (no SD) } \\ \text { 5. Convalescent faecal carriage } \\ \text { 6. Length of hospitalization (no SD) } \\ \text { 7. Serious adverse events } \\ \text { 8. Other adverse events }\end{array}$

Notes Location: Italy

Date: 1991-3

Severity of illness at entry: all severe 
Dolecek 2008

Methods Generation of allocation sequence: computer-generated, block randomization

Allocation concealment: sealed envelopes

Blinding: open

Inclusion of all randomized culture-positive participants in the final analysis: $268 / 288$ (93\%)

Participants 285 analysed $^{a}: 145$ in gatifloxacin group; 140 in azithromycin group

Adult and children inpatients aged 1 to 41 years (210/287 (73\%) participants below the age of 15 years)

Inclusion criteria: clinical or culture-positive enteric fever

Exclusion criteria: no consent; pregnancy; age < 6 months; history of hypersensitivity to either of the trial drugs; any signs of severe typhoid fever or previous reported treatment with a fluoroquinolone antibiotics; a third-generation cephalosporin or macrolide antibiotic within 1 week before to hospital admission

Interventions 1. Gatifloxacin $(10 \mathrm{mg} / \mathrm{kg} /$ day oral once daily for 7 days $)$

2. Azithromycin ( $20 \mathrm{mg} / \mathrm{kg} /$ day oral once daily for 7 days)

Outcomes 1. Clinical failure

2. Microbiological failure

3. Relapse

4. Fever clearance time

5. Complications

6. Length of hospitalization

7. Convalescent faecal carriage

8. Serious adverse events

9. Other adverse events

Notes Location: Vietnam (multi-centre, 3 hospitals)

Date: 2004-5

Severity of illness at entry: all uncomplicated

Received as an unpublished trial (with additional data), but reference updated to current citation upon publication

\section{Duong 1995}

Methods Generation of allocation sequence: random-number table

Allocation concealment: sealed envelopes

Blinding: open

Inclusion of all randomized culture-positive participants in the final analysis: 40/63 (63\%)

Participants 63 analysed $^{a}: 22$ in 3-day group; 41 in 5-day group

Adult inpatients aged 15 to 65 years

Inclusion criteria: clinical

Exclusion criteria: pregnant or lactating; hypersensitivity to nalidixic acid and derivatives; history of cerebral disorders;

severe concomitant disease; complications of enteric fever; received any fluoroquinolone in previous week

Interventions 1. Fleroxacin (400 mg oral once daily for 3 days)

2. Fleroxacin ( $400 \mathrm{mg}$ oral once daily for 5 days)

Outcomes 1. Clinical failure

2. Microbiological failure

Fluoroquinolones for treating typhoid and paratyphoid fever (enteric fever) (Review)

Copyright () 2008 The Cochrane Collaboration. Published by John Wiley \& Sons, Ltd. 
Duong 1995 (Continued)

\begin{tabular}{|c|c|}
\hline & $\begin{array}{l}\text { 3. Relapse } \\
\text { 4. Fever clearance time } \\
\text { 5. Complications } \\
\text { 6. Length of hospitalization } \\
\text { 7. Serious adverse events } \\
\text { 8. Other adverse events (number of events stated) }\end{array}$ \\
\hline Notes & $\begin{array}{l}\text { Location: Vietnam } \\
\text { Date: } 1993-4 \\
\text { Severity of illness at entry: all uncomplicated } \\
\text { Author provided further information }\end{array}$ \\
\hline
\end{tabular}

Flores 1994

\begin{tabular}{ll}
\hline Methods & $\begin{array}{l}\text { Generation of allocation sequence: unclear } \\
\text { Allocation concealment: unclear } \\
\text { Blinding: open } \\
\text { Inclusion of all randomized culture-positive participants in the final analysis: } 100 \%\end{array}$ \\
\hline Participants & $\begin{array}{l}40 \text { analysed } \text { : } 20 \text { in ofloxacin group; } 20 \text { in ampicillin group } \\
\text { Adults (abstract keyword); most probably inpatients } \\
\text { Inclusion criteria: clinical with culture positive } \\
\text { Exclusion criteria: not reported }\end{array}$ \\
\hline Interventions & $\begin{array}{l}\text { 1. Ampicillin }(1 \text { g every } 6 \text { hours for } 10 \text { days) } \\
\text { 2. Ofloxacin (400 mg every } 12 \text { hours for } 10 \text { days })\end{array}$ \\
\hline Outcomes & $\begin{array}{l}\text { 1. Clinical failure } \\
\text { 2. Microbiological failure } \\
\text { 3. Serious adverse events }\end{array}$ \\
\hline Notes & $\begin{array}{l}\text { Location: Mexico } \\
\text { Date: not reported } \\
\text { Severity of illness at entry: not reported } \\
\text { Abstract only }\end{array}$ \\
\hline
\end{tabular}

Gasem 2003

Methods Generation of allocation sequence: random-number table

Allocation concealment: sealed envelopes

Blinding: open

Inclusion of all randomized culture-positive participants in the final analysis: $100 \%$

Participants 55 analysed $^{a}: 28$ in ciprofloxacin group; 27 in chloramphenicol group

Adult inpatients

Inclusion criteria: clinical and $\geq 14$ years

Exclusion criteria: severe complications; treatment with chloramphenicol, ciprofloxacin, other fluoroquinolones before admission; history of allergy to chloramphenicol/quinolone; malaria or other infection; white blood cell count 
Gasem 2003 (Continued)

$<2000 / \mathrm{mL}$; pregnant or lactating

$\begin{array}{ll}\text { Interventions } & \text { 1. Ciprofloxacin (500 mg twice daily for } 7 \text { days) } \\ & \text { 2. Chloramphenicol (500 mg oral } 4 \text { times a day for } 14 \text { days) }\end{array}$

Outcomes 1. Clinical failure

2. Microbiological failure

3. Relapse

4. Fever clearance time

5. Complications

6. Length of hospitalization

7. Serious adverse events

8. Other adverse events

Notes Location: Indonesia

Date: not reported

Severity of illness at entry: none had severe complications on enrolment

\section{Girgis 1999}

Methods Generation of allocation sequence: random-number list, block randomization

Allocation concealment: sealed envelopes

Blinding: open

Inclusion of all randomized culture-positive participants in the final analysis: $100 \%$

Participants 64 analysed $^{a}: 28$ in ciprofloxacin group; 36 in azithromycin group

Adult inpatients aged $>18$ years

Inclusion criteria: clinical

Exclusion criteria: pregnant or lactating; allergy to ciprofloxacin or erythromycin/other macrolides; those with complications of typhoid fever; inability to swallow medications; significant underlying illness; treatment within past 4 days with an antibiotic with potential efficacy against $S$. Typhi

Interventions 1. Ciprofloxacin (500 mg oral twice daily for 7 days)

2. Azithromycin ( $1 \mathrm{~g}$ oral once daily for the first day followed by oral $500 \mathrm{mg}$ once daily for total duration of 7 days)

Outcomes 1. Clinical failure

2. Microbiological failure

3. Relapse

4. Fever clearance time

5. Complications

6. Length of hospitalization

7. Cost of treatment

8. Convalescent faecal carriage

9. Serious adverse events

10. Other adverse events (number of events stated)

Fluoroquinolones for treating typhoid and paratyphoid fever (enteric fever) (Review)

Copyright @ 2008 The Cochrane Collaboration. Published by John Wiley \& Sons, Ltd. 


\section{Girgis 1999 (Continued)}

\begin{tabular}{ll}
\hline Notes & Location: Egypt \\
& Date: $1997-8$ \\
& Severity of illness at entry: all uncomplicated \\
& Author provided further information \\
\hline
\end{tabular}

\section{Gottuzzo 1992}

Methods Generation of allocation sequence: unclear

Allocation concealment: unclear

Blinding: double

Inclusion of all randomized culture-positive participants in the final analysis: 95/98 (97\%)

Participants 98 analysed $^{a}: 49$ in ciprofloxacin group; 49 in chloramphenicol group

Adult inpatients

Inclusion criteria: clinical with culture positive for $S$. Typhi or $S$. Paratyphi

Interventions 1. Ciprofloxacin (500 mg oral every 12 hours for 10 days)

2. Chloramphenicol (750 mg oral every 6 hours for 14 days)

\begin{tabular}{ll} 
Outcomes & $\begin{array}{l}\text { 1. Clinical failure } \\
\text { 2. Relapse }\end{array}$ \\
\hline Notes & $\begin{array}{l}\text { Location: not available } \\
\text { Date: not available } \\
\text { Severity of illness at entry: not reported }\end{array}$
\end{tabular}

\section{Hajji 1988}

Methods Generation of allocation sequence: random-number table

Allocation concealment: sealed envelopes

Blinding: open

Inclusion of all randomized culture-positive participants in the final analysis: $100 \%$

Participants 42 analysed $^{a}: 24$ in pefloxacin group; 18 in co-trimoxazole group

Adult inpatients aged $>16$ years

Inclusion criteria: clinical

Exclusion criteria: not reported

Interventions 1. Pefloxacin (400 mg oral twice daily for 14 days)

2. Co-trimoxazole (160/800 mg oral twice daily for 14 days)

5 participants were given intravenous pefloxacin for mean 4.8 days; 4 were given intramuscular co-trimoxazole for mean 6 days

Outcomes
1. Clinical failure
2. Microbiological failure
3. Relapse
4. Fever clearance time (no SD, non-exact P value) 


\section{Hajji 1988 (Continued)}

\begin{tabular}{|c|c|}
\hline & $\begin{array}{l}\text { 5. Complications } \\
\text { 6. Convalescent faecal carriage } \\
\text { 7. Serious adverse events } \\
\text { 8. Other adverse events }\end{array}$ \\
\hline Notes & $\begin{array}{l}\text { Location: Morocco } \\
\text { Date: } 1984-5 \\
\text { Severity of illness at entry: comatose or neurological disorders in } 3 \text { participants in pefloxacin group and } 2 \text { participants } \\
\text { in co-trimoxazole group } \\
\text { Author provided further information }\end{array}$ \\
\hline
\end{tabular}

Huai 2000

\begin{tabular}{ll} 
Methods & $\begin{array}{l}\text { Generation of allocation sequence: unclear } \\
\text { Allocation concealment: unclear } \\
\text { Blinding: open } \\
\text { Inclusion of all randomized culture-positive participants in the final analysis: } 100 \%\end{array}$ \\
\hline Participants & $\begin{array}{l}60 \text { analysed } \text { : } 30 \text { in norfloxacin group; } 30 \text { in ceftriaxone group } \\
\text { Children inpatients } \\
\text { Inclusion criteria: clinical with blood or bone marrow positive for } S \text {. Typhi } \\
\text { Exclusion criteria: not mentioned }\end{array}$ \\
\hline Interventions & $\begin{array}{l}\text { 1. Norfloxacin (10 to } 20 \mathrm{mg} / \mathrm{kg} / \text { day oral divided in } 2 \text { times per day until afebrile, no drug for } 5 \text { days, and administer } \\
\text { again for } 5 \text { days) } \\
\text { 2. Ceftriaxone (100 mg/kg/day intravenous until afebrile, no drug for } 5 \text { days, and administer again for } 5 \text { days) }\end{array}$ \\
\hline Outcomes & $\begin{array}{l}\text { 1. Clinical failure } \\
\text { 2. Relapse } \\
\text { 3. Fever clearance time } \\
\text { 4. Other adverse events }\end{array}$ \\
\hline Notes & $\begin{array}{l}\text { Location: China (Chinese language) } \\
\text { Date: } 1995-8 \\
\text { Severity of illness at entry: norfloxacin group had } 7 \text { participants with liver damage and } 4 \text { with myocardial damage; } \\
\text { and ceftriaxone group had } 8 \text { participants with liver damage and } 3 \text { with myocardial damage }\end{array}$ \\
\hline & \\
&
\end{tabular}

Jia 1994

Methods Generation of allocation sequence: unclear

Allocation concealment: unclear

Blinding: double

Inclusion of all randomized culture-positive participants in the final analysis: $100 \%$

Participants 130 analysed $^{a}: 63$ in pefloxacin group; 67 in norfloxacin group

Age not mentioned (adult dosages used); inpatients

Inclusion criteria: clinical with culture positive blood or bone marrow for $S$. Typhi

Exclusion criteria: not mentioned 
Interventions 1. Pefloxacin ( $400 \mathrm{mg}$ plus placebo oral twice daily for 10 to 14 days)

2. Norfloxacin (300 mg plus placebo oral 3 times a day for 10 to 14 days)

\begin{tabular}{l|l} 
Outcomes & 1. Clinical failure \\
& 2. Fever clearance time \\
& 3. Complications \\
4. Serious adverse events
\end{tabular}

Notes Location: China (Chinese language)

Date: $1991-2$

Severity of illness at entry: not reported

Both groups in Jia 1994 were the same as Weng 1996. Weng 1996 did not report use of placebo as Jia 1994 did. Weng 1996 also included other groups. Due to the ambiguity surrounding the use of placebo in the norfloxacin group, we decided to make Jia 1994 the primary reference because the methodology was clearer in this publication

Kalo 1997

\begin{tabular}{ll}
\hline Methods & $\begin{array}{l}\text { Generation of allocation sequence: unclear } \\
\text { Allocation concealment: unclear } \\
\text { Blinding: open } \\
\text { Inclusion of all randomized culture-positive participants in the final analysis: } 100 \%\end{array}$ \\
\hline Participants & $\begin{array}{l}\text { 30 analysed }{ }^{a}: 15 \text { in } 7 \text {-day group; } 15 \text { in } 10 \text {-day group } \\
\text { Adult inpatients aged } 16 \text { to } 42 \text { years } \\
\text { Inclusion criteria: blood-culture positive; ampicillin-resistant } S . \text { Typhi } \\
\text { Exclusion criteria: received quinolones within } 2 \text { weeks before hospitalization }\end{array}$ \\
\hline Interventions & $\begin{array}{l}\text { 1. Perfloxacin (400 mg oral twice daily for } 7 \text { days) } \\
\text { 2. Perfloxacin (400 mg oral twice daily for } 10 \text { days) }\end{array}$ \\
\hline Outcomes & $\begin{array}{l}\text { 1. Clinical failure } \\
\text { 2. Microbiological failure } \\
\text { 3. Relapse } \\
\text { 4. Convalescent faecal carriage } \\
\text { 5. Serious adverse events }\end{array}$ \\
\hline Notes & $\begin{array}{l}\text { Location: Albania } \\
\text { Date: } 1992-4 \\
\text { Severity of illness at entry: not reported }\end{array}$ \\
\hline
\end{tabular}


Limson 1989

Methods Generation of allocation sequence: random-number table

Allocation concealment: unclear

Blinding: open

Inclusion of all randomized culture-positive participants in the final analysis: $100 \%$

Participants 40 analysed $^{a}: 20$ in ciprofloxacin group; 20 in co-trimoxazole group

Adult inpatients aged 18 to 77 years

Inclusion criteria: clinical

Exclusion criteria: complications; drug allergy; renal impairment

Interventions 1. Ciprofloxacin (500 mg oral twice daily for 10 days)

2. Co-trimoxazole (160/800 mg oral twice daily for 14 days)

$\begin{array}{ll}\text { Outcomes } & \text { 1. Clinical failure } \\ & \text { 2. Microbiological failure } \\ & \text { 3. Serious adverse events } \\ \text { 4. Other adverse events }\end{array}$

Notes Location: Philippines

Date: not reported

Severity of illness at entry: all uncomplicated

\section{Morelli 1992}

Methods Generation of allocation sequence: computer-generated

Allocation concealment: unclear

Blinding: open

Inclusion of all randomized culture-positive participants in the final analysis: $100 \%$

Participants 156 analysed $^{a}: 30$ each in ofloxacin and chloramphenicol groups; 36 in pefloxacin group; 20 each in ciprofloxacin, enoxacin, and norfloxacin groups

Adult inpatients aged 16 to 60 years

Inclusion criteria: blood culture positive for $S$. Typhi; high fever for not more than 5 days; toxic symptomatology

Exclusion criteria: hypersensitivity or allergy to fluoroquinolone or antibiotic treatment

Interventions 1 . Ofloxacin (300 mg oral every 8 hours for 15 days)

2. Pefloxacin ( $400 \mathrm{mg}$ oral every 8 hours for 15 days)

3. Ciprofloxacin ( $500 \mathrm{mg}$ oral every 8 hours for 15 days)

4. Enoxacin (300 mg oral every 8 hours for 15 days)

5. Norfloxacin ( $400 \mathrm{mg}$ oral every 8 hours for 15 days)

6. Chloramphenicol (500 mg oral every 6 hours for 15 days)

Outcomes 1. Clinical failure

2. Relapse

3. Fever clearance time (no SD)

4. Convalescent faecal carriage

5. Other adverse events (number of events stated)

Fluoroquinolones for treating typhoid and paratyphoid fever (enteric fever) (Review)

Copyright ( 2008 The Cochrane Collaboration. Published by John Wiley \& Sons, Ltd. 


\section{Morelli 1992 (Continued)}

\begin{tabular}{l|l}
\hline Notes & Location: Italy \\
& Date: $1985-90$ \\
& Severity of illness at entry: not reported \\
& We prepared different comparisons with these data: a combination of all 5 fluoroquinolone groups vs the chloram- \\
& phenicol group; and norfloxacin vs ofloxacin, pefloxacin, and enoxacin \\
\hline
\end{tabular}

\section{Nalin 1987}

Methods Generation of allocation sequence: randomized numbers

Allocation concealment: unclear

Blinding: open

Inclusion of all randomized culture-positive participants in the final analysis: $100 \%$

Participants 169 analysed $^{a}: 90$ in norfloxacin group; 79 in chloramphenicol group

Age not mentioned (adult dosages used); most probably inpatients

Inclusion criteria: clinical

Exclusion criteria: pregnant; prior antibiotic

Interventions 1. Chloramphenicol ( $500 \mathrm{mg}$ every 4 to 6 hours for 14 days)

2. Norfloxacin ( $400 \mathrm{mg}$ every 8 hours for 14 days)

\begin{tabular}{l|l} 
Outcomes & 1. Clinical failure \\
& 2. Microbiological failure \\
& 3. Fever clearance time \\
4. Convalescent faecal carriage \\
5. Other adverse events
\end{tabular}

Notes Location: multicentre in Chile, Guatemala, Mexico, and Peru

Date: not reported

Severity of illness at entry: all uncomplicated

\section{Nguyen 1997}

Methods Generation of allocation sequence: block randomization

Allocation concealment: sealed envelopes

Blinding: open

Inclusion of all randomized culture-positive participants in the final analysis: 49/101 (49\%)

Participants 100 analysed $^{a}: 47$ in 2-day group; 53 in 3-day group

Adult inpatients aged $>15$ years

Inclusion criteria: clinical

Exclusion criteria: pregnant; severe disease requiring intensive care; known hypersensitivity to quinolones; received treatment with quinolones in the week before admission or responded to ampicillin, chloramphenicol, or co-trimoxazole

Interventions 1 . Ofloxacin $(15 \mathrm{mg} / \mathrm{kg} / \mathrm{day}$ oral for 2 days $)$

2. Ofloxacin $(10 \mathrm{mg} / \mathrm{kg} /$ day oral for 3 days $)$

Fluoroquinolones for treating typhoid and paratyphoid fever (enteric fever) (Review)

Copyright ( 2008 The Cochrane Collaboration. Published by John Wiley \& Sons, Ltd. 
Nguyen 1997 (Continued)

\begin{tabular}{l|l}
\hline Outcomes & 1. Clinical failure \\
& 2. Microbiological failure \\
& 3. Relapse \\
& 4. Fever clearance time \\
& 5. Complications \\
& 6. Length of hospitalization \\
& 7. Convalescent faecal carriage \\
8. Serious adverse events
\end{tabular}

\section{Pandit 2007}

Methods Generation of allocation sequence: computer-generated, block randomization

Allocation concealment: sealed envelopes

Blinding: open

Inclusion of all randomized culture-positive participants in the final analysis: $147 / 169$ (87\%)

Participants 158 analysed $^{a}: 88$ in gatifloxacin group; 70 in cefixime group

Adults and children outpatients aged 2.75 to 50 years $(60 / 169(35.5 \%)$ were children aged $<14$ years)

Inclusion criteria: clinical

Exclusion criteria: not residing $2.5 \mathrm{~km}$ radius from hospital; age not between 2 to 65 years; not willing to give informed consent; not able to take oral medications; pregnant or lactating; history of seizures; not able to stay in city for treatment duration; known contraindication to cephalosporins or fluoroquinolones; complicated typhoid fever or received third-generation cephalosporins, fluoroquinolones, or macrolide in week before presentation to clinic

Interventions 1. Gatifloxacin $(10 \mathrm{mg} / \mathrm{kg} /$ day in single dose oral for 7 days)

2. Cefixime $(20 \mathrm{mg} / \mathrm{kg} /$ day in 2 divided doses oral for 7 days)

$\begin{array}{ll}\text { Outcomes } & \text { 1. Clinical failure } \\ & \text { 2. Microbiological failure } \\ & \text { 3. Relapse } \\ & \text { 4. Fever clearance time } \\ & \text { 5. Convalescent faecal carriage } \\ & \text { 6. Complications } \\ & \text { 7. Serious adverse events } \\ \text { 8 Other adverse events }\end{array}$

Fluoroquinolones for treating typhoid and paratyphoid fever (enteric fever) (Review)

Copyright ( 2008 The Cochrane Collaboration. Published by John Wiley \& Sons, Ltd. 
Methods Generation of allocation sequence: computer-generated

Allocation concealment: sealed envelopes

Blinding: open

Inclusion of all randomized culture-positive participants in the final analysis: $114 / 130$ (88\%)

Participants 125 analysed $^{a}: 63$ in ofloxacin group; 62 in azithromycin group

Adults and children inpatients 3 to 42 years $(87 \%$ (163/187) were children < 15 years for all three arms)

Inclusion criteria: clinical

Exclusion criteria: severe or complicated disease; inability to swallow oral medications; history of significant underlying disease or hypersensitivity to either of trial drugs; pregnant or lactating; history of treatment with fluoroquinolones

or expanded spectrum cephalosporins; macrolide within 1 week of hospital admission

Interventions 1. Ofloxacin $(20 \mathrm{mg} / \mathrm{kg} /$ day in 2 divided doses oral for 7 days $)$

2. Azithromycin $(10 \mathrm{mg} / \mathrm{kg} /$ day once a day oral for 7 days $)$

Comparison not included in this review:

3. Ofloxacin-azithromycin ( $15 \mathrm{mg} / \mathrm{kg} /$ day in 2 divided doses oral ofloxacin for 7 days and $10 \mathrm{mg} / \mathrm{kg} /$ day once a day oral azithromycin for first 3 days)

Outcomes 1. Clinical failure

2. Microbiological failure

3. Relapse

4. Fever clearance time (mean and 95\% confidence intervals; SD calculated by review author)

5. Complications

6. Length of hospitalization (mean and 95\% confidence intervals; SD calculated by review author)

7. Convalescent faecal carriage

8. Serious adverse events

9. Other adverse events (numbers not stated)

Notes Location: Vietnam

Date: 1998-2002

Severity of illness at entry: all uncomplicated

Author provided further information

\section{Phongmany 2005}

Methods Generation of allocation sequence: random-number table, block randomization

Allocation concealment: sealed envelopes

Blinding: open

Inclusion of all randomized culture-positive participants in the final analysis: 48/50 (96\%)

Participants 50 analysed $^{a}: 27$ in ofloxacin group; 23 in chloramphenicol group

Adult inpatients aged $>15$ years

Inclusion criteria: clinical or blood culture positive typhoid fever

Exclusion criteria: age $\leq 15$ years; pregnant; lactating; not able to take oral medication; not willing to give informed consent; not able to stay in hospital for the duration of treatment; known to have contraindications to chloramphenicol or ofloxacin; severe typhoid fever; or intractable vomiting 
Phongmany 2005 (Continued)

\begin{tabular}{ll} 
Interventions & $\begin{array}{l}\text { 1. Ofloxacin }(15 \mathrm{mg} / \mathrm{kg} / \text { day in } 2 \text { divided doses oral for } 3 \text { days }) \\
\text { 2. Chloramphenicol }(50 \mathrm{mg} / \mathrm{kg} / \text { day oral in } 4 \text { divided doses for } 14 \text { days })\end{array}$ \\
\hline Outcomes & $\begin{array}{l}\text { 1. Clinical failure } \\
\text { 2. Fever clearance time } \\
\text { 3. Complications } \\
\text { 4. Length of hospitalization } \\
\text { 5. Serious adverse events } \\
\text { 6. Other adverse events }\end{array}$ \\
\hline Notes & $\begin{array}{l}\text { Location: Laos } \\
\text { Date: } 2001-3 \\
\text { Severity of illness at entry: all uncomplicated } \\
\text { Author provided further information }\end{array}$ \\
\hline
\end{tabular}

\section{Quintero 1988}

\begin{tabular}{ll}
\hline Methods & $\begin{array}{l}\text { Generation of allocation sequence: unclear } \\
\text { Allocation concealment: unclear } \\
\text { Blinding: double } \\
\text { Inclusion of all randomized culture-positive participants in the final analysis: } 100 \%\end{array}$ \\
\hline Participants & $\begin{array}{l}26 \text { analysed }{ }^{a}: 13 \text { in ciprofloxacin group; } 13 \text { in chloramphenicol group } \\
\text { Age not mentioned (adult dosages used); most probably inpatients } \\
\text { Inclusion criteria: not reported } \\
\text { Exclusion criteria: not reported }\end{array}$ \\
\hline Interventions & $\begin{array}{l}\text { 1. Ciprofloxacin (750 mg oral } 3 \text { times a day for unknown duration) } \\
\text { 2. Chloramphenicol (750 mg oral } 4 \text { times a day for unknown duration) }\end{array}$ \\
\hline Outcomes & $\begin{array}{l}\text { 1. Clinical failure } \\
\text { 2. Fever clearance time } \\
\text { 3. Serious adverse events }\end{array}$ \\
\hline Notes & $\begin{array}{l}\text { Location: Mexico } \\
\text { Date: not reported } \\
\text { Severity of illness at entry: not reported } \\
\text { Conference abstract }\end{array}$ \\
\hline
\end{tabular}

\section{Sarma 1991}

Methods Generation of allocation sequence: randomized numbers

Allocation concealment: unclear

Blinding: open

Inclusion of all randomized culture-positive participants in the final analysis: $100 \%$ 


\section{Sarma 1991 (Continued)}

\begin{tabular}{ll} 
Participants & $\begin{array}{l}40 \text { analysed }^{a}: 20 \text { participants in norfloxacin group; } 20 \text { participants in chloramphenicol group } \\
\text { Adult inpatients aged } 17 \text { to } 32 \text { years } \\
\text { Inclusion criteria: clinical with blood-culture positive for } S \text {. Typhi or } S \text {. Paratyphi } \\
\text { Exclusion criteria: complications of typhoid fever; pregnancy; previous antibiotic; known allergy to norfloxacin or } \\
\text { chloramphenicol }\end{array}$ \\
\hline Interventions & $\begin{array}{l}\text { 1. Norfloxacin ( } 400 \mathrm{mg} \text { oral every } 12 \text { hours for } 7 \text { days) } \\
\text { 2. Chloramphenicol }(60 \mathrm{mg} / \mathrm{kg} / \text { day oral in } 4 \text { divided doses until afebrile then } 40 \mathrm{mg} / \mathrm{kg} / \text { day in } 3 \text { divided doses to } \\
\text { complete } 14 \text { days) }\end{array}$ \\
\hline Outcomes & $\begin{array}{l}\text { 1. Clinical failure } \\
\text { 2. Microbiological failure } \\
\text { 3. Relapse } \\
\text { 4. Fever clearance time } \\
\text { 5. Complications } \\
\text { 6. Length of hospitalization } \\
\text { 7. Convalescent faecal carriage } \\
\text { 8. Serious adverse events } \\
\text { 9. Other adverse events }\end{array}$ \\
\hline $\begin{array}{l}\text { Location: India } \\
\text { Date: } 1990 \\
\text { Severity of illness at entry: all uncomplicated } \\
\text { Author provided further information }\end{array}$ \\
\hline Notes
\end{tabular}

\section{Smith 1994}

Methods Generation of allocation sequence: computer-generated

Allocation concealment: sealed envelopes

Blinding: open

Inclusion of all randomized culture-positive participants in the final analysis: $50 \%$

Participants 47 analysed $^{a}: 22$ in ofloxacin group; 25 in ceftriaxone group

Adult inpatients aged 15 to 63 years

Inclusion criteria: clinical or culture positive for enteric fever

Exclusion criteria: hypersensitivity to beta-lactam antibiotics or quinolones; previous treatment with broad-spectrum cephalosporins or quinolone within 1 week of hospital admission; those who responded to ampicillin, chloramphenicol, or co-trimoxazole

Interventions 1 . Ofloxacin (200 mg oral every 12 hours for 5 days)

2. Ceftriaxone ( $3 \mathrm{~g}$ intravenous once a day for 3 days)

Outcomes
1. Clinical failure
2. Microbiological failure
3. Relapse
4. Fever clearance time
5. Complications
6. Length of hospitalization (mean and range)

Fluoroquinolones for treating typhoid and paratyphoid fever (enteric fever) (Review) 
Smith 1994 (Continued)

7. Convalescent faecal carriage

8. Serious adverse events

9. Other adverse events

Notes Location: Vietnam

Date: $1992-3$

Severity of illness at entry: all uncomplicated

Author provided further information

Tran 1994

\begin{tabular}{ll}
\hline Methods & $\begin{array}{l}\text { Generation of allocation sequence: computer-generated } \\
\text { Allocation concealment: sealed envelopes } \\
\text { Blinding: open } \\
\text { Inclusion of all randomized culture-positive participants in the final analysis: } 15 / 31(48.4 \%)\end{array}$ \\
\hline Participants & $\begin{array}{l}\text { 31 analysed }{ }^{a}: 16 \text { in fleroxacin group; } 15 \text { in ceftriaxone group } \\
\text { Adult inpatients aged } \geq 16 \text { years } \\
\text { Inclusion criteria: clinical with a negative malaria blood film }\end{array}$ \\
\hline Interventions & $\begin{array}{l}\text { 1. Fleroxacin (400 mg oral for } 7 \text { days) } \\
\text { 2. Ceftriaxone (2 g intravenous for } 5 \text { days) }\end{array}$ \\
\hline Outcomes & $\begin{array}{l}\text { 1. Clinical failure } \\
\text { 2. Microbiological failure } \\
\text { 3. Relapse } \\
\text { 4. Fever clearance time } \\
\text { 5. Complications } \\
\text { 6. Convalescent faecal carriage } \\
\text { 7. Serious adverse events }\end{array}$ \\
\hline Notes & $\begin{array}{l}\text { Location: Vietnam } \\
\text { Date: } 1992-3 \\
\text { Severity of illness at entry: all uncomplicated } \\
\text { Author provided further information }\end{array}$ \\
\hline
\end{tabular}

\section{Tran 1995}

Methods Generation of allocation sequence: computer-generated

Allocation concealment: sealed envelopes

Blinding: open

Inclusion of all randomized culture-positive participants in the final analysis: 50\% (114/228)

Participants 228 analysed $^{a}: 118$ in 3-day group; 110 in 5-day group

Adults and children outpatients (180 culture positive were aged $<17$ years)

Inclusion criteria: clinical

Exclusion criteria: unable to take oral medications due to vomiting; severe disease; shock; impaired consciousness;

bleeding; peritonitis; pregnant; neonates; received a fluoroquinolone 
Tran 1995 (Continued)

\begin{tabular}{ll}
\hline Interventions & $\begin{array}{l}\text { 1. Ofloxacin }(15 \mathrm{mg} / \mathrm{kg} / \text { day oral for } 3 \text { days }) \\
\text { 2. Ofloxacin }(10 \mathrm{mg} / \mathrm{kg} / \text { day oral for } 5 \text { days })\end{array}$ \\
\hline Outcomes & $\begin{array}{l}\text { 1. Clinical failure } \\
\text { 2. Microbiological failure } \\
\text { 3. Relapse } \\
\text { 4. Fever clearance time } \\
\text { 5. Convalescent faecal carriage } \\
\text { 6. Serious adverse events } \\
\text { 7. Other adverse events }\end{array}$ \\
\hline Notes & $\begin{array}{l}\text { Location: Vietnam } \\
\text { Date: } 1993-3 \\
\text { Severity of illness at entry: all uncomplicated } \\
\text { Author provided further information }\end{array}$ \\
\hline
\end{tabular}

\section{Unal 1996}

Methods Generation of allocation sequence: unclear

Allocation concealment: unclear

Blinding: open

Inclusion of all randomized culture-positive participants in the final analysis: $100 \%$

Participants 46 analysed $^{a}: 22$ in 5-day group; 24 in 7-day group

Adult inpatients aged $>16$ years

Inclusion criteria: febrile disease; 1 or more blood and/or bone marrow culture positive for Salmonella species

Exclusion criteria: age < 16 years; pregnant or lactating; jaundice; hepatic failure; antibiotics in the last 2 weeks

Interventions 1. Pefloxacin ( $400 \mathrm{mg}$ oral twice daily for 5 days)

2. Pefloxacin ( $400 \mathrm{mg}$ oral twice daily for 7 days)

Outcomes $\quad$ 1. Clinical failure

2. Microbiological failure

3. Relapse

4. Fever clearance time

5. Convalescent faecal carriage

6. Serious adverse events

7. Other adverse events

Notes Location: Turkey

Date: $1992-4$

Severity of illness at entry: not reported 
Methods Generation of allocation sequence: computer-generated

Allocation concealment: sealed envelopes

Blinding: open

Inclusion of all randomized culture-positive participants in the final analysis: $26 / 100$ (26\%)

\begin{tabular}{|c|c|}
\hline Participants & $\begin{array}{l}100 \text { analysed }^{a}: 53 \text { in } 2 \text {-day grou } \\
\text { Children inpatients aged } 1 \text { to } 1 \\
\text { Inclusion criteria: clinical or blo } \\
\text { Exclusion criteria: severe diseas } \\
\text { testinal bleed, clinical signs of in } \\
\text { to fluoroquinolones; received an }\end{array}$ \\
\hline Interventions & $\begin{array}{l}\text { 1. Ofloxacin }(15 \mathrm{mg} / \mathrm{kg} / \text { day ora } \\
\text { 2. Ofloxacin }(15 \mathrm{mg} / \mathrm{kg} / \text { day ora }\end{array}$ \\
\hline Outcomes & $\begin{array}{l}\text { 1. Clinical failure } \\
\text { 2. Microbiological failure } \\
\text { 3. Relapse } \\
\text { 4. Fever clearance time } \\
\text { 5. Complications } \\
\text { 6. Length of hospitalization } \\
\text { 7. Convalescent faecal carriage } \\
\text { 8. Serious adverse events } \\
\text { 9. Other adverse events }\end{array}$ \\
\hline
\end{tabular}

Notes Location: Vietnam

Date: not reported

Severity of illness at entry: all uncomplicated

Author provided further information

Vinh 2005

Methods Generation of allocation sequence: computer-generated

Allocation concealment: sealed envelopes

Blinding: open

Inclusion of all randomized culture-positive participants in the final analysis: 196/202 (97\%)

Participants 196 analysed $^{a}: 89$ in ofloxacin 2-day group; 107 in ofloxacin 3-day group

Children inpatients aged $<15$ years

Inclusion criteria: clinical

Exclusion criteria: no informed consent from parent or guardian; previous treatment active against $S$. Typhi or $S$. Paratyphi (but those with no response to chloramphenicol, ampicillin, or co-trimoxazole were included); severe or complicated disease

Interventions 1. Ofloxacin $(10 \mathrm{mg} / \mathrm{kg} /$ day oral in 2 divided doses for 2 days)

2. Ofloxacin ( $10 \mathrm{mg} / \mathrm{kg} /$ day oral in 2 divided doses for 3 days)

Fluoroquinolones for treating typhoid and paratyphoid fever (enteric fever) (Review)

Copyright ( 2008 The Cochrane Collaboration. Published by John Wiley \& Sons, Ltd. 
Vinh 2005 (Continued)

\begin{tabular}{ll} 
Outcomes & 1. Clinical failure \\
& 2. Microbiological failure \\
3. Relapse \\
4. Fever clearance time (mean and $95 \%$ confidence intervals; SD calculated by review author) \\
5. Complications \\
6. Length of hospitalization (mean and $95 \%$ confidence intervals; SD calculated by review author) \\
7. Convalescent faecal carriage \\
8. Serious adverse events \\
9. Other adverse events \\
\hline Notes \\
Location: Vietnam \\
Date: $1994-6$ \\
Severity of illness at entry: all uncomplicated \\
Author provided further information
\end{tabular}

\section{Wallace 1993}

Methods Generation of allocation sequence: unclear

Allocation concealment: unclear

Blinding: open

Inclusion of all randomized culture-positive participants in the final analysis: $41 / 42$ (97.6\%)

Participants 42 analysed $^{a}: 20$ in ciprofloxacin group; 22 in ceftriaxone group

Adult inpatients

Inclusion criteria: blood culture positive for $S$. Typhi

Exclusion criteria: only positive Widal and/or a positive stool culture; age < 16 years; unable to take oral medications; possible proven pregnancy; and lack of fever at admission

Interventions 1. Ciprofloxacin (500 mg oral twice daily for 7 days)

2. Ceftriaxone ( $3 \mathrm{~g} /$ day intravenous for 7 days)

$\begin{array}{ll}\text { Outcomes } & \text { 1. Clinical failure } \\ & \text { 2. Microbiological failure } \\ \text { 3. Relapse } \\ \text { 4. Fever clearance time (SD not reported) } \\ \text { 5. Convalescent faecal carriage } \\ \text { 6. Complications }\end{array}$

Notes Location: Bahrain

Date: not reported

Severity of illness at entry: not reported 


\begin{tabular}{ll} 
Methods & $\begin{array}{l}\text { Generation of allocation sequence: unclear } \\
\text { Allocation concealment: unclear } \\
\text { Blinding: open } \\
\text { Inclusion of all randomized culture-positive participants in the final analysis: } 100 \%\end{array}$ \\
\hline Participants & $\begin{array}{l}23 \text { analysed } a \text { : } 8 \text { in norfloxacin group; } 6 \text { in pefloxacin group; and } 9 \text { in ofloxacin group } \\
\text { Adult and children inpatients aged } 11 \text { to } 62 \text { years } \\
\text { Inclusion criteria: clinical with blood or bone marrow culture positive for } S . \text { Typhi } \\
\text { Exclusion criteria: not mentioned }\end{array}$ \\
\hline Interventions & $\begin{array}{l}\text { We evaluated } 3 \text { of the available } 5 \text { groups: } \\
\text { 1. Norfloxacin (300 to } 400 \text { mg oral thrice a day for } 14 \text { days) } \\
\text { 2. Pefloxacin (400 mg oral twice daily for } 14 \text { days) } \\
\text { 3. Ofloxacin (300 mg oral twice daily for } 14 \text { days) }\end{array}$ \\
\hline Outcomes & $\begin{array}{l}\text { 1. Clinical failure } \\
\text { 2. Fever clearance time }\end{array}$ \\
\hline Notes & $\begin{array}{l}\text { Location: China (Chinese language) } \\
\text { Date: not reported } \\
\text { Severity of illness at entry: some participants had complications }\end{array}$ \\
\hline
\end{tabular}

\section{Yang 1991}

Methods Generation of allocation sequence: unclear

Allocation concealment: unclear

Blinding: open

Inclusion of all randomized culture-positive participants in the final analysis: $100 \%$

Participants 56 analysed $^{a}: 28$ in ofloxacin group; 28 in norfloxacin group

Inpatients; mean age 27 years (standard deviation 14 years) in ofloxacin group; mean 21 years (standard deviation 10 years) in norfloxacin group

Inclusion criteria: clinical with blood culture positive for $S$. Typhi

Exclusion criteria: not mentioned

Interventions 1 . Ofloxacin (200 $\mathrm{mg}$ oral twice daily for 7 to 14 days)

2. Norfloxacin (300 to $400 \mathrm{mg}$ oral 3 to 4 times a day for 10 to 24 days)

Outcomes 1. Clinical failure

2. Microbiological failure (definition incorrect, thus we did not enter data in this review)

3. Relapse

4. Fever clearance time

5. Convalescent faecal carriage

6. Serious adverse events

7. Other adverse events

Notes Location: China (Chinese language)

Date: $1989-91$

Severity of illness at entry: not reported 
Yousaf 1992

\begin{tabular}{ll} 
Methods & $\begin{array}{l}\text { Generation of allocation sequence: unclear } \\
\text { Allocation concealment: unclear } \\
\text { Blinding: open } \\
\text { Inclusion of all randomized culture-positive participants in the final analysis: } 75 / 85(88.4 \%)\end{array}$ \\
\hline Participants & $\begin{array}{l}75 \text { analysed }^{a}: 25 \text { in ofloxacin group; } 25 \text { in chloramphenicol group; } 25 \text { in amoxicillin group } \\
\text { Adult inpatients } \\
\text { Inclusion criteria: culture positive } \\
\text { Exclusion criteria: if received previous antibiotic therapy known to be effective against } S . \text { Typhi }\end{array}$ \\
\hline Interventions & $\begin{array}{l}\text { 1. Ofloxacin (200 mg oral twice daily for } 14 \text { days) } \\
\text { 2. Chloramphenicol }(50 \mathrm{mg} / \mathrm{kg} / \text { day, then } 30 \mathrm{mg} / \mathrm{kg} / \text { day when afebrile for } 14 \text { days }) \\
\text { 3. Amoxicillin }(4 \text { to } 6 \mathrm{~g} / \mathrm{day} \text { oral for } 14 \text { days })\end{array}$ \\
\hline Outcomes & $\begin{array}{l}\text { 1. Clinical failure } \\
\text { 2. Microbiological failure } \\
\text { 3. Other adverse events }\end{array}$ \\
\hline Notes & $\begin{array}{l}\text { Location: Pakistan } \\
\text { Date: } 1989-92 \\
\text { Severity of illness at entry: not reported }\end{array}$ \\
\hline
\end{tabular}

\section{Yu 1998}

\begin{tabular}{ll} 
Methods & $\begin{array}{l}\text { Generation of allocation sequence: unclear } \\
\text { Allocation concealment: unclear } \\
\text { Blinding: open } \\
\text { Inclusion of all randomized culture-positive participants in the final analysis: } 100 \%\end{array}$ \\
\hline Participants & $\begin{array}{l}80 \text { analysed }^{a}: 40 \text { in levofloxacin group; } 40 \text { in cefixime group } \\
\text { Adult aged } 18 \text { to } 65 \text { years; most probably inpatients } \\
\text { Inclusion criteria: clinical with blood or bone marrow culture positive for } S . \text { Typhi or } S . \text { Paratyphi } \\
\text { Exclusion criteria: not mentioned }\end{array}$ \\
\hline Interventions & $\begin{array}{l}\text { 1. Levofloxacin (200 mg oral twice a day for } 10 \text { days) } \\
\text { 2. Cefixime (200 mg oral twice a day for } 10 \text { days) }\end{array}$ \\
\hline Outcomes & $\begin{array}{l}\text { 1. Clinical failure } \\
\text { 2. Microbiological failure } \\
\text { 3. Relapse } \\
\text { 4. Fever clearance time } \\
\text { 5. Complications } \\
\text { 6. Convalescent faecal carriage } \\
\text { 7. Other adverse events }\end{array}$ \\
\hline
\end{tabular}

Notes Location: China (Chinese language)

Date: not reported 
Yu 1998 (Continued)

Severity of illness at entry: included 'mild, common, and severe' types (1 'severe type' illness in levofloxacin group and 2 in cefixime group)

MDR: multiple-drug resistant; SD: standard deviation; S. Typhi/Paratyphi: Salmonella enterica serotype Typhi/Paratyphi.

${ }^{a}$ For details of number of participants enrolled, number randomized, and the number of participants with culture-confirmed enteric fever, see Table 1: Microbiology.

\section{Characteristics of excluded studies [ordered by study ID]}

\begin{tabular}{|c|c|}
\hline Agalar 1997 & $\begin{array}{l}\text { Not a randomized controlled trial because } 1 \text { group consisted of participants admitted in } 1994 \text { and the other } \\
\text { group of participants admitted in } 1995\end{array}$ \\
\hline Akhtar 1989 & No mention of randomization \\
\hline Akhtar 1992 & $\begin{array}{l}\text { Quasi-randomized controlled trial: participants were allocated alternatively to either ciprofloxacin group or } \\
\text { chloramphenicol group, and resistance strains assigned to a third ciprofloxacin group; author provided this } \\
\text { additional information }\end{array}$ \\
\hline Bavdekar 1991 & Interventions not randomly assigned \\
\hline Bethell 1996 & $\begin{array}{l}\text { Children from the Vinh } 1996 \text { trial (which is included in this review) were entered into this pharmacokinetic } \\
\text { study of oral vs intravenous ofloxacin }\end{array}$ \\
\hline Chakravorty 1991 & All treated with chloramphenicol; some switched over to another drug based on culture results \\
\hline Chukwani 1998 & $\begin{array}{l}2 \text { fluoroquinolone drugs were given for different durations ( } 7 \text { days vs } 14 \text { days) in this randomized controlled } \\
\text { trial }\end{array}$ \\
\hline Daga 1994 & Treatment assigned depending on treatment already taken, clinical course, and complications \\
\hline Hou 1993 & Randomized controlled trial comparing Chinese ofloxacin with Japanese ofloxacin \\
\hline Jinlong 1998 & Quasi-randomized controlled trial \\
\hline Kumar 2007 & $\begin{array}{l}\text { Described as a randomized controlled parallel study of ofloxacin vs ceftriaxone in } 93 \text { children with multi- } \\
\text { drug resistant typhoid fever proven by blood culture. The main outcome reported for both arms is mean fever } \\
\text { clearance time; however the number of children in each arm is not available. We have contacted the author for } \\
\text { additional information (December 2007) and will include this study if further information becomes available }\end{array}$ \\
\hline Liberti 2000 & No mention of randomization \\
\hline Lu 1995 & $\begin{array}{l}\text { A total of } 130 \text { participants with any infectious disease were randomized into } 2 \text { groups (enoxacin and cefotaxime) } \\
\text {; there were only } 2 \text { participants with enteric fever in enoxacin group and } 1 \text { participant with enteric fever in } \\
\text { cefotaxime group }\end{array}$ \\
\hline
\end{tabular}

Fluoroquinolones for treating typhoid and paratyphoid fever (enteric fever) (Review)

Copyright ( 2008 The Cochrane Collaboration. Published by John Wiley \& Sons, Ltd. 
(Continued)

\begin{tabular}{|c|c|}
\hline Nelwan 1995 & $\begin{array}{l}\text { Randomized controlled trial comparing } 3 \text { days with } 6 \text { days of ciprofloxacin that included } 20 \text { participants with } \\
\text { serologically confirmed enteric fever (of a total of } 59 \text { participants randomized). We contacted the author (17 } \\
\text { December 2003) to obtain additional data for blood culture confirmed cases and will include this in future } \\
\text { updates should it become available }\end{array}$ \\
\hline Peyramond 1986 & Not a randomized controlled trial \\
\hline Secmeer 1997 & No randomization; allocation based on co-trimoxazole susceptibility \\
\hline Singh 1993 & No mention of randomization \\
\hline Suhendro 2007 & $\begin{array}{l}\text { Compares } 2 \text { different formulations of ciprofloxacin; described as a prospective, open labelled, clinical trial, } \\
\text { comparing safety and efficacy of extended-release ciprofloxacin } 1000 \mathrm{mg} \text { once daily (Ciprofloxacin XR) and } \\
\text { ciprofloxacin intermediate release } 500 \mathrm{mg} 2 \text { times daily (Ciprofloxacin bid) in adults with typhoid fever }\end{array}$ \\
\hline Takkar 1994 & Not randomized \\
\hline Tanphaichitra 1986 & $\begin{array}{l}\text { Randomized controlled trial of gonorrhoea; part of the report, but not part of the trial, were } 8 \text { participants } \\
\text { with enteric fever that treated with ofloxacin }\end{array}$ \\
\hline Uwaydah 1992 & Compares 2 ciprofloxacin doses, not durations \\
\hline Wain 1997 & $\begin{array}{l}\text { Study on S. Typhi isolates from blood cultures of participants included in } 3 \text { trials included in this review: } \\
\text { Smith 1994; Vinh 1996; and Nguyen } 1997\end{array}$ \\
\hline Zavala 1989 & No mention of randomization \\
\hline Zhang 1991 & $\begin{array}{l}\text { Randomized controlled trial including several infections; randomization not applied to the } 63 \text { typhoid partic- } \\
\text { ipants treated with enoxacin }\end{array}$ \\
\hline ZhongYang 1997 & $\begin{array}{l}\text { Randomized controlled trial comparing ofloxacin with norfloxacin for } 14 \text { days. It included } 158 \text { people with } \\
\text { serologically confirmed enteric fever, out of a total of } 429 \text { people randomized. Microbiological failure, the } \\
\text { only reported outcome for blood culture confirmed cases, was measured at different times for both arms ( } 7 \\
\text { to } 14 \text { days for the ofloxacin group vs } 7 \text { days for the norfloxacin group). We contacted the author (July 2003) } \\
\text { to obtain additional data for blood culture-confirmed cases and will include this in future updates should it } \\
\text { become available }\end{array}$ \\
\hline
\end{tabular}

S. Typhi: Salmonella enterica serotype Typhi. 


\section{Characteristics of studies awaiting assessment [ordered by study ID]}

Flores 1991

\begin{tabular}{ll}
\hline Methods & NA \\
\hline Participants & NA \\
\hline Interventions & NA \\
\hline Outcomes & NA \\
\hline Notes & Unable to retrieve this study \\
\hline
\end{tabular}

Soewandojo 1992

\begin{tabular}{ll}
\hline Methods & NA \\
\hline Participants & NA \\
\hline Interventions & NA \\
\hline Outcomes & NA \\
\hline Notes & Unable to retrieve this study \\
\hline
\end{tabular}

NA: not available.

\section{Characteristics of ongoing studies [ordered by study ID]}

\section{ISRCTN53258327}

Trial name or title

"An open randomised study to assess the efficacy of gatifloxacin versus chloramphenicol for the treatment of uncomplicated typhoid fever in Kathmandu, Nepal"

Methods "open randomised study”

Participants

Inclusion criteria: any patient with suspected uncomplicated enteric fever who gives consent Exclusion criteria: no consent, or pregnant

Interventions $\quad$ 1. Gatifloxacin $10 \mathrm{mg} / \mathrm{kg} /$ day once a day for 7 days

2. Chloramphenicol $75 \mathrm{mg} / \mathrm{kg} /$ day in 4 divided doses for 14 days

Outcomes

1. Failure of treatment, defined as occurrence of any 1 of: persistent fever at day 10 of treatment; failure to clear completely the admission symptoms at day 10; blood culture positive at day 10 of treatment; need for 'rescue' treatment with ceftriaxone; culture-confirmed relapse within 28 days of starting therapy; development on treatment of any complication (clinically significant bleeding, fall in the Glasgow Coma Score, perforation of the gastrointestinal tract, admission to hospital within 28 days of starting therapy) 
ISRCTN53258327 (Continued)

\begin{tabular}{|c|c|}
\hline Starting date & $\begin{array}{l}1 \text { May } 2006 \\
\text { Anticipated end date: } 30 \text { June } 2008\end{array}$ \\
\hline Contact information & Dr Buddha Basnyat (rishibas@wlink.com.np), Patan Hospital, Kathmandu, Nepal \\
\hline Notes & $\begin{array}{l}\text { Location: Nepal } \\
\text { Registration number: ISRCTN } 53258327 \\
\text { Source of funding: The Wellcome Trust (UK) } \\
\text { Note: trial was stopped temporarily in September } 2006 \text { after the reports of dysglycaemia associated with } \\
\text { gatifloxacin, but it was resumed in December } 2006 \\
\text { Percentage of children aged < } 14 \text { years in trial: around } 45 \% \text { of } 703 \text { enrolled participants }\end{array}$ \\
\hline
\end{tabular}

\section{ISRCTN66534807}

Trial name or title "A randomised clinical trial of Azithromycin versus Ofloxacin in the treatment of adults with uncomplicated typhoid fever at Mahosot Hospital, Vientiane, Lao People’s Democratic Republic (PDR)”

\begin{tabular}{|c|c|}
\hline Methods & "randomised clinical trial" \\
\hline Participants & $\begin{array}{l}\text { Inclusion criteria: adult ( } \geq 15 \text { years) non-pregnant patients with suspected or blood-culture proven typhoid; } \\
\text { fever }>37.5^{\circ} \mathrm{C} \text {; informed written consent to the study; able to stay in hospital for } 7 \text { days; able to take oral } \\
\text { medication; bodyweight }>40 \mathrm{~kg} \text {; likely to be able to complete } 6 \text { months' follow up; none of the exclusion } \\
\text { criteria } \\
\text { Exclusion criteria: known hypersensitivity to ofloxacin or azithromycin; administration of chloramphenicol, } \\
\text { co-trimoxazole, ampicillin, azithromycin, or a fluoroquinolone during previous week; pregnancy or breast- } \\
\text { feeding; contraindications to ofloxacin or azithromycin; evidence for severe typhoid }\end{array}$ \\
\hline Interventions & $\begin{array}{l}\text { 1. Ofloxacin } 7.5 \mathrm{mg} / \mathrm{kg} \text { every } 12 \text { hours for } 3 \text { days } \\
\text { 2. Azithromycin } 20 \mathrm{mg} / \mathrm{kg} \text { every } 24 \text { hours for } 3 \text { days }\end{array}$ \\
\hline Outcomes & $\begin{array}{l}\text { 1. Fever clearance time } \\
\text { 2. Cure } \\
\text { 3. Relapse } \\
\text { 4. Faecal carriage }\end{array}$ \\
\hline Starting date & $\begin{array}{l}1 \text { May } 2004 \\
\text { Anticipated end date: } 31 \text { December } 2007\end{array}$ \\
\hline Contact information & $\begin{array}{l}\text { Dr Paul Newton (paul@tropmedres.ac), Microbiology laboratory, Ministry of Health, Mahosot Hospital, } \\
\text { Vientiane, Laos }\end{array}$ \\
\hline Notes & $\begin{array}{l}\text { Location: Laos } \\
\text { Registration number: ISRCTN66534807 } \\
\text { Source of funding: The Wellcome Trust (UK) } \\
\text { Percentage of children in trial: none } \\
\text { E-mail update by Dr Newton on } 5 \text { December 2007: on hold because of considerable decline in incidence of } \\
\text { typhoid in Vientiane }\end{array}$ \\
\hline
\end{tabular}

Fluoroquinolones for treating typhoid and paratyphoid fever (enteric fever) (Review)

Copyright ( 2008 The Cochrane Collaboration. Published by John Wiley \& Sons, Ltd. 
DATA AND ANALYSES

\section{Comparison 1. Fluoroquinolones vs chloramphenicol}

\begin{tabular}{|c|c|c|c|c|}
\hline Outcome or subgroup title & $\begin{array}{l}\text { No. of } \\
\text { studies }\end{array}$ & $\begin{array}{c}\text { No. of } \\
\text { participants }\end{array}$ & Statistical method & Effect size \\
\hline 1 Clinical failure & 9 & 594 & Odds Ratio (M-H, Fixed, 95\% CI) & $0.65[0.25,1.72]$ \\
\hline $\begin{array}{l}1.1 \mathrm{NaR} \text { not reported and } \\
\text { MDR absent }\end{array}$ & 5 & 307 & Odds Ratio (M-H, Fixed, 95\% CI) & $0.81[0.19,3.34]$ \\
\hline $\begin{array}{l}1.2 \mathrm{NaR} \text { absent and MDR } \\
\text { present }\end{array}$ & 1 & 50 & Odds Ratio (M-H, Fixed, 95\% CI) & $0.27[0.01,7.02]$ \\
\hline $\begin{array}{l}1.3 \mathrm{NaR} \text { and MDR not } \\
\text { reported }\end{array}$ & 3 & 237 & Odds Ratio (M-H, Fixed, 95\% CI) & $0.65[0.14,2.91]$ \\
\hline 2 Microbiological failure & 6 & 378 & Odds Ratio (M-H, Fixed, 95\% CI) & $0.43[0.18,1.03]$ \\
\hline $\begin{array}{l}2.1 \mathrm{NaR} \text { not reported and } \\
\text { MDR absent }\end{array}$ & 4 & 237 & Odds Ratio (M-H, Fixed, 95\% CI) & $0.67[0.21,2.12]$ \\
\hline $\begin{array}{l}2.2 \mathrm{NaR} \text { and MDR not } \\
\text { reported }\end{array}$ & 2 & 141 & Odds Ratio (M-H, Fixed, 95\% CI) & $0.24[0.06,0.96]$ \\
\hline 3 Relapse & 6 & 467 & Odds Ratio (M-H, Fixed, 95\% CI) & $0.14[0.04,0.50]$ \\
\hline $\begin{array}{l}3.1 \mathrm{NaR} \text { not reported and } \\
\text { MDR absent }\end{array}$ & 4 & 281 & Odds Ratio (M-H, Fixed, 95\% CI) & $0.12[0.02,0.69]$ \\
\hline $\begin{array}{l}3.2 \mathrm{NaR} \text { and } \mathrm{MDR} \text { not } \\
\text { reported }\end{array}$ & 2 & 186 & Odds Ratio (M-H, Fixed, 95\% CI) & $0.17[0.03,1.07]$ \\
\hline 4 Fever clearance time & 3 & 129 & Mean Difference (IV, Fixed, 95\% CI) & $\begin{array}{l}-25.93[-40.12,- \\
11.74]\end{array}$ \\
\hline $\begin{array}{l}\text { 4.1 NaR not reported and } \\
\text { MDR absent }\end{array}$ & 2 & 81 & Mean Difference (IV, Fixed, 95\% CI) & $\begin{array}{l}-16.07[-35.03 \\
2.88]\end{array}$ \\
\hline $\begin{array}{l}4.2 \mathrm{NaR} \text { absent and MDR } \\
\text { present }\end{array}$ & 1 & 48 & Mean Difference (IV, Fixed, 95\% CI) & $\begin{array}{l}-38.5[-59.90,- \\
17.10]\end{array}$ \\
\hline 5 Length of hospital stay & 2 & 105 & Mean Difference (IV, Fixed, 95\% CI) & $-2.57[-3.53,-1.62]$ \\
\hline $\begin{array}{l}\text { 5.1 NaR not reported and } \\
\text { MDR absent }\end{array}$ & 1 & 55 & Mean Difference (IV, Fixed, 95\% CI) & $-0.40[-1.63,0.83]$ \\
\hline $\begin{array}{l}5.2 \mathrm{NaR} \text { absent and MDR } \\
\text { present }\end{array}$ & 1 & 50 & Mean Difference (IV, Fixed, 95\% CI) & $-5.9[-7.42,-4.38]$ \\
\hline $\begin{array}{l}6 \text { Convalescent faecal carriage } \\
\text { (NaR not reported and MDR } \\
\text { absent) }\end{array}$ & 3 & 298 & Odds Ratio (M-H, Fixed, 95\% CI) & $0.17[0.04,0.70]$ \\
\hline 7 Complications & 2 & 105 & Odds Ratio (M-H, Fixed, 95\% CI) & $0.71[0.16,3.05]$ \\
\hline $\begin{array}{l}\text { 7.1 NaR not reported and } \\
\text { MDR absent }\end{array}$ & 1 & 55 & Odds Ratio (M-H, Fixed, 95\% CI) & $0.96[0.18,5.23]$ \\
\hline $\begin{array}{l}7.2 \mathrm{NaR} \text { absent and MDR } \\
\text { present }\end{array}$ & 1 & 50 & Odds Ratio (M-H, Fixed, 95\% CI) & $0.27[0.01,7.02]$ \\
\hline 8 Adverse events (not serious) & 5 & 245 & Odds Ratio (M-H, Fixed, 95\% CI) & $1.10[0.46,2.62]$ \\
\hline $\begin{array}{l}\text { 8.1 NaR not reported and } \\
\text { MDR absent }\end{array}$ & 3 & 145 & Odds Ratio (M-H, Fixed, 95\% CI) & $1.32[0.47,3.73]$ \\
\hline $\begin{array}{l}\text { 8.2 NaR not reported and } \\
\text { MDR not reported }\end{array}$ & 1 & 50 & Odds Ratio (M-H, Fixed, 95\% CI) & $0.72[0.14,3.59]$ \\
\hline
\end{tabular}




\section{Comparison 2. Fluoroquinolones vs amoxicillin (AMX) or ampicillin (AMP)}

\begin{tabular}{lcccc} 
Outcome or subgroup title & $\begin{array}{c}\text { No. of } \\
\text { studies }\end{array}$ & $\begin{array}{c}\text { No. of } \\
\text { participants }\end{array}$ & Statistical method & Effect size \\
\hline $\begin{array}{c}1 \text { Clinical failure (MDR and NaR } \\
\text { not reported) }\end{array}$ & 2 & 90 & Odds Ratio (M-H, Fixed, 95\% CI) & $0.08[0.01,0.46]$ \\
$\begin{array}{c}2 \text { Microbiological failure (MDR } \\
\text { and NaR not reported) }\end{array}$ & 2 & 90 & Odds Ratio (M-H, Fixed, 95\% CI) & $0.10[0.02,0.58]$ \\
$\begin{array}{c}\text { Adverse events (not serious) } \\
\text { (MDR and NaR not reported) }\end{array}$ & 1 & & Odds Ratio (M-H, Fixed, 95\% CI) & Totals not selected \\
\hline
\end{tabular}

\section{Comparison 3. Fluoroquinolones vs co-trimoxazole}

\begin{tabular}{|c|c|c|c|c|}
\hline Outcome or subgroup title & $\begin{array}{l}\text { No. of } \\
\text { studies }\end{array}$ & $\begin{array}{c}\text { No. of } \\
\text { participants }\end{array}$ & Statistical method & Effect size \\
\hline 1 Clinical failure (MDR absent) & 2 & 82 & Odds Ratio (M-H, Fixed, 95\% CI) & $0.18[0.01,4.01]$ \\
\hline $1.1 \mathrm{NaR}$ absent & 1 & 42 & Odds Ratio (M-H, Fixed, 95\% CI) & Not estimable \\
\hline $1.2 \mathrm{NaR}$ not reported & 1 & 40 & Odds Ratio (M-H, Fixed, 95\% CI) & $0.18[0.01,4.01]$ \\
\hline $\begin{array}{l}2 \text { Microbiological failure (MDR } \\
\text { absent) }\end{array}$ & 2 & 82 & Odds Ratio (M-H, Fixed, 95\% CI) & $0.18[0.01,4.01]$ \\
\hline $2.1 \mathrm{NaR}$ absent & 1 & 42 & Odds Ratio (M-H, Fixed, 95\% CI) & Not estimable \\
\hline 2.2 NaR not reported & 1 & 40 & Odds Ratio (M-H, Fixed, 95\% CI) & $0.18[0.01,4.01]$ \\
\hline $\begin{array}{l}3 \text { Adverse events (not serious) } \\
\quad \text { (MDR absent) }\end{array}$ & 2 & 82 & Odds Ratio (M-H, Fixed, 95\% CI) & $0.77[0.22,2.69]$ \\
\hline $3.1 \mathrm{NaR}$ absent & 1 & 42 & Odds Ratio (M-H, Fixed, 95\% CI) & $0.74[0.04,12.67]$ \\
\hline 3.2 NaR not reported & 1 & 40 & Odds Ratio (M-H, Fixed, 95\% CI) & $0.78[0.19,3.13]$ \\
\hline
\end{tabular}

\section{Comparison 4. Fluoroquinolones vs azithromycin}

\begin{tabular}{lcccc} 
Outcome or subgroup title & $\begin{array}{c}\text { No. of } \\
\text { studies }\end{array}$ & $\begin{array}{c}\text { No. of } \\
\text { participants }\end{array}$ & Statistical method & Effect size \\
\hline 1 Clinical failure (in adults) & 2 & 152 & Odds Ratio (M-H, Fixed, 95\% CI) & $3.32[0.63,17.43]$ \\
$1.1 \mathrm{NaR}$ present & 1 & 88 & Odds Ratio (M-H, Fixed, 95\% CI) & $3.32[0.63,17.43]$ \\
$\begin{array}{l}1.2 \mathrm{NaR} \text { not reported } \\
\text { Microbiological failure (in }\end{array}$ & 1 & 64 & Odds Ratio (M-H, Fixed, 95\% CI) & $\begin{array}{l}\text { Not estimable } \\
\text { adults) }\end{array}$ \\
$\begin{array}{l}2.1 \mathrm{NaR} \text { present } \\
\text { 2.2 NaR not reported }\end{array}$ & 1 & 152 & Odds Ratio (M-H, Fixed, 95\% CI) & $2.05[0.18,23.44]$ \\
\\
$\begin{array}{l}\text { Fluoroquinolones for treating typhoid and paratyphoid fever (enteric fever) (Review) } \\
\text { Copyright } \odot \text { 2008 The Cochrane Collaboration. Published by John Wiley \& Sons, Ltd. }\end{array}$
\end{tabular}




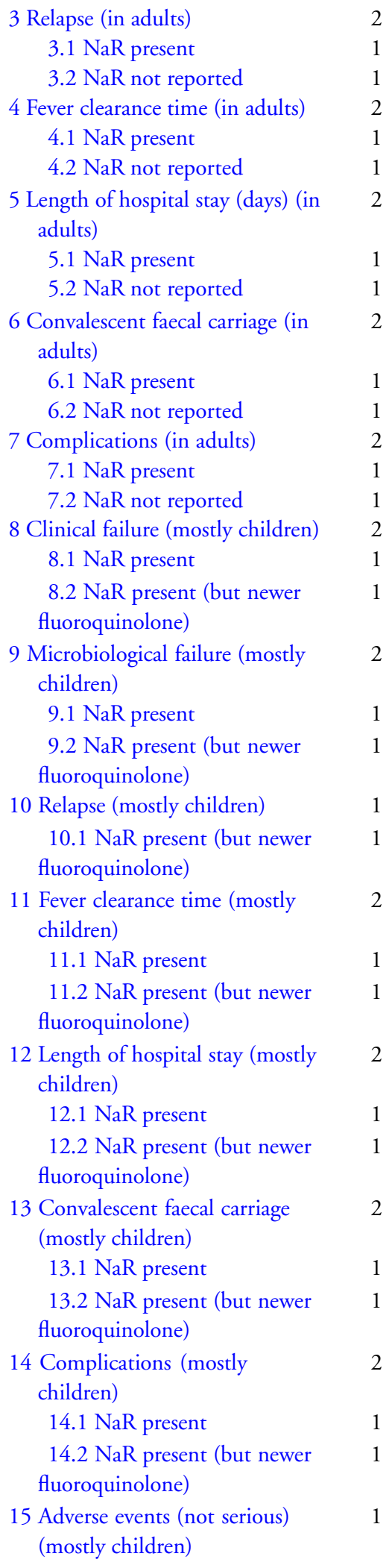

$6.94[0.31,154.85]$

$6.94[0.31,154.85]$

Not estimable

$-8.95[-20.09,2.19]$

$4.0[-21.50,29.50]$

$-12.0[-24.39,0.39]$

$0.90[-0.32,2.12]$

$0.90[-0.32,2.12]$

Not estimable

$21.33[1.18,386.00]$

$21.33[1.18,386.00]$

Not estimable

$1.0[0.06,16.51]$

$1.0[0.06,16.51]$

Not estimable

Totals not selected

Not estimable

Not estimable

Totals not selected

Not estimable

Not estimable

Totals not selected

Not estimable

Totals not selected

Not estimable

Not estimable

Totals not selected

Not estimable

Not estimable

Totals not selected

Not estimable

Not estimable

Totals not selected

Not estimable

Not estimable

Totals not selected 


\begin{tabular}{|c|c|c|c|c|}
\hline Outcome or subgroup title & $\begin{array}{l}\text { No. of } \\
\text { studies }\end{array}$ & $\begin{array}{c}\text { No. of } \\
\text { participants }\end{array}$ & Statistical method & Effect size \\
\hline $\begin{array}{l}1 \text { Clinical failure (adults or mostly } \\
\text { adults) }\end{array}$ & 2 & 238 & Odds Ratio (M-H, Fixed, 95\% CI) & $0.05[0.01,0.24]$ \\
\hline $1.1 \mathrm{NaR}$ not reported & 1 & 80 & Odds Ratio (M-H, Fixed, 95\% CI) & $0.13[0.01,2.65]$ \\
\hline $\begin{array}{l}1.2 \mathrm{NaR} \text { present (but newer } \\
\text { fluoroquinolone) }\end{array}$ & 1 & 158 & Odds Ratio (M-H, Fixed, 95\% CI) & $0.03[0.00,0.24]$ \\
\hline $\begin{array}{l}2 \text { Microbiological failure (adults } \\
\text { or mostly adults) }\end{array}$ & 2 & 238 & Odds Ratio (M-H, Fixed, 95\% CI) & $0.14[0.02,1.23]$ \\
\hline $2.1 \mathrm{NaR}$ not reported & 1 & 80 & Odds Ratio (M-H, Fixed, 95\% CI) & $0.10[0.01,1.92]$ \\
\hline $\begin{array}{l}2.2 \mathrm{NaR} \text { present (but newer } \\
\text { fluoroquinolone) }\end{array}$ & 1 & 158 & Odds Ratio (M-H, Fixed, 95\% CI) & $0.26[0.01,6.53]$ \\
\hline 3 Relapse (adults or mostly adults) & 2 & 218 & Odds Ratio (M-H, Fixed, 95\% CI) & $0.18[0.03,0.91]$ \\
\hline $3.1 \mathrm{NaR}$ not reported & 1 & 80 & Odds Ratio (M-H, Fixed, 95\% CI) & Not estimable \\
\hline $\begin{array}{l}3.2 \mathrm{NaR} \text { present (but newer } \\
\text { fluoroquinolone) }\end{array}$ & 1 & 138 & Odds Ratio (M-H, Fixed, 95\% CI) & $0.18[0.03,0.91]$ \\
\hline $\begin{array}{l}4 \text { Fever clearance time (adults or } \\
\text { mostly adults) }\end{array}$ & 2 & 238 & Mean Difference (IV, Fixed, 95\% CI) & $\begin{array}{l}-41.69[-54.96,- \\
28.42]\end{array}$ \\
\hline $4.1 \mathrm{NaR}$ not reported & 1 & 80 & Mean Difference (IV, Fixed, 95\% CI) & $\begin{array}{l}-36.0[-51.29,- \\
20.71]\end{array}$ \\
\hline $\begin{array}{l}\text { 4.2 NaR present (but newer } \\
\text { fluoroquinolone) }\end{array}$ & 1 & 158 & Mean Difference (IV, Fixed, 95\% CI) & $\begin{array}{l}-57.00[-85.68,- \\
32.32]\end{array}$ \\
\hline $\begin{array}{l}5 \text { Convalescent faecal carriage } \\
\text { (adults or mostly adults) }\end{array}$ & 2 & 227 & Odds Ratio (M-H, Fixed, 95\% CI) & $0.26[0.01,6.50]$ \\
\hline $5.1 \mathrm{NaR}$ not reported & 1 & 80 & Odds Ratio (M-H, Fixed, 95\% CI) & Not estimable \\
\hline $\begin{array}{l}5.2 \mathrm{NaR} \text { present (but newer } \\
\text { fluoroquinolone) }\end{array}$ & 1 & 147 & Odds Ratio (M-H, Fixed, 95\% CI) & $0.26[0.01,6.50]$ \\
\hline $\begin{array}{l}6 \text { Complications (adults or mostly } \\
\text { adults) }\end{array}$ & 1 & & Odds Ratio (M-H, Fixed, 95\% CI) & Totals not selected \\
\hline $\begin{array}{l}\text { 6.1 NaR present (but newer } \\
\text { fluoroquinolone) }\end{array}$ & 1 & & Odds Ratio (M-H, Fixed, 95\% CI) & Not estimable \\
\hline $\begin{array}{l}7 \text { Adverse events (not serious) } \\
\text { (adults or mostly adults) }\end{array}$ & 2 & 238 & Odds Ratio (M-H, Random, 95\% CI) & $3.30[0.11,97.30]$ \\
\hline 7.1 NaR not reported & 1 & 80 & Odds Ratio (M-H, Random, 95\% CI) & $0.65[0.10,4.11]$ \\
\hline $\begin{array}{l}7.2 \mathrm{NaR} \text { present (but newer } \\
\text { fluoroquinolone) }\end{array}$ & 1 & 158 & Odds Ratio (M-H, Random, 95\% CI) & $17.74[2.30,136.58]$ \\
\hline 8 Clinical failure (children only) & 1 & & Odds Ratio (M-H, Fixed, 95\% CI) & Totals not selected \\
\hline $8.1 \mathrm{NaR}$ absent & 1 & & Odds Ratio (M-H, Fixed, 95\% CI) & Not estimable \\
\hline $\begin{array}{l}9 \text { Microbiological failure (children } \\
\text { only) }\end{array}$ & 1 & & Odds Ratio (M-H, Fixed, 95\% CI) & Totals not selected \\
\hline $9.1 \mathrm{NaR}$ absent & 1 & & Odds Ratio (M-H, Fixed, 95\% CI) & Not estimable \\
\hline 10 Relapse (children only) & 1 & & Odds Ratio (M-H, Fixed, 95\% CI) & Totals not selected \\
\hline $10.1 \mathrm{NaR}$ absent & 1 & & Odds Ratio (M-H, Fixed, 95\% CI) & Not estimable \\
\hline
\end{tabular}




\begin{tabular}{|c|c|c|c|}
\hline $\begin{array}{l}11 \text { Fever clearance time (children } \\
\text { only) }\end{array}$ & 1 & Mean Difference (IV, Fixed, 95\% CI) & Totals not selected \\
\hline $11.1 \mathrm{NaR}$ absent & 1 & Mean Difference (IV, Fixed, 95\% CI) & Not estimable \\
\hline $\begin{array}{l}12 \text { Length of hospital stay } \\
\text { (children only) }\end{array}$ & 1 & Mean Difference (IV, Fixed, 95\% CI) & Totals not selected \\
\hline $12.1 \mathrm{NaR}$ absent & 1 & Mean Difference (IV, Fixed, 95\% CI) & Not estimable \\
\hline 13 Complications (children only) & 1 & Odds Ratio (M-H, Fixed, 95\% CI) & Totals not selected \\
\hline 13.1 NaR absent & 1 & Odds Ratio (M-H, Fixed, 95\% CI) & Not estimable \\
\hline
\end{tabular}

\section{Comparison 6. Fluoroquinolones vs ceftriaxone}

\begin{tabular}{|c|c|c|c|c|}
\hline Outcome or subgroup title & $\begin{array}{l}\text { No. of } \\
\text { studies }\end{array}$ & $\begin{array}{c}\text { No. of } \\
\text { participants }\end{array}$ & Statistical method & Effect size \\
\hline 1 Clinical failure & 3 & 120 & Odds Ratio (M-H, Fixed, 95\% CI) & $0.08[0.01,0.45]$ \\
\hline $1.1 \mathrm{NaR}$ absent (adults only) & 1 & 47 & Odds Ratio (M-H, Fixed, 95\% CI) & $0.07[0.00,1.26]$ \\
\hline $\begin{array}{l}1.2 \mathrm{NaR} \text { not reported (adults } \\
\text { only) }\end{array}$ & 2 & 73 & Odds Ratio (M-H, Fixed, 95\% CI) & $0.09[0.01,0.76]$ \\
\hline 2 Microbiological failure & 3 & 119 & Odds Ratio (M-H, Fixed, 95\% CI) & $0.32[0.03,3.17]$ \\
\hline $2.1 \mathrm{NaR}$ absent & 1 & 47 & Odds Ratio (M-H, Fixed, 95\% CI) & $0.36[0.01,9.37]$ \\
\hline $2.2 \mathrm{NaR}$ not reported & 2 & 72 & Odds Ratio (M-H, Fixed, 95\% CI) & $0.27[0.01,7.25]$ \\
\hline 3 Relapse & 3 & 81 & Odds Ratio (M-H, Fixed, 95\% CI) & $0.34[0.03,3.47]$ \\
\hline 3.1 NaR absent (adults only) & 1 & 23 & Odds Ratio (M-H, Fixed, 95\% CI) & $0.33[0.01,9.07]$ \\
\hline $\begin{array}{l}3.2 \mathrm{NaR} \text { not reported (adults } \\
\text { only) }\end{array}$ & 2 & 58 & Odds Ratio (M-H, Fixed, 95\% CI) & $0.35[0.01,9.08]$ \\
\hline 4 Fever clearance time & 2 & 76 & Mean Difference (IV, Fixed, 95\% CI) & $\begin{array}{l}-101.20[-129.21,- \\
73.19]\end{array}$ \\
\hline 4.1 NaR absent (adults only) & 1 & 47 & Mean Difference (IV, Fixed, 95\% CI) & $\begin{array}{l}-113.00[-150.67,- \\
79.33]\end{array}$ \\
\hline $\begin{array}{l}\text { 4.2 NaR not reported (adults } \\
\text { only) }\end{array}$ & 1 & 29 & Mean Difference (IV, Fixed, 95\% CI) & $\begin{array}{l}-79.0[-124.24,- \\
33.76]\end{array}$ \\
\hline 5 Convalescent faecal carriage & 3 & 81 & Odds Ratio (M-H, Fixed, 95\% CI) & $0.35[0.01,9.08]$ \\
\hline $5.1 \mathrm{NaR}$ absent & 1 & 23 & Odds Ratio (M-H, Fixed, 95\% CI) & Not estimable \\
\hline $5.2 \mathrm{NaR}$ not reported & 2 & 58 & Odds Ratio (M-H, Fixed, 95\% CI) & $0.35[0.01,9.08]$ \\
\hline 6 Complications & 3 & 120 & Odds Ratio (M-H, Fixed, 95\% CI) & $0.21[0.01,4.59]$ \\
\hline 6.1 NaR absent & 1 & 47 & Odds Ratio (M-H, Fixed, 95\% CI) & $0.21[0.01,4.59]$ \\
\hline $6.2 \mathrm{NaR}$ not reported & 2 & 73 & Odds Ratio (M-H, Fixed, 95\% CI) & Not estimable \\
\hline 7 Serious adverse events & 2 & 78 & Odds Ratio (M-H, Fixed, 95\% CI) & $0.29[0.01,7.76]$ \\
\hline $7.1 \mathrm{NaR}$ absent & 1 & 47 & Odds Ratio (M-H, Fixed, 95\% CI) & Not estimable \\
\hline $7.2 \mathrm{NaR}$ not reported & 1 & 31 & Odds Ratio (M-H, Fixed, 95\% CI) & $0.29[0.01,7.76]$ \\
\hline 8 Adverse events (not serious) & 1 & & Odds Ratio (M-H, Fixed, 95\% CI) & Totals not selected \\
\hline $8.1 \mathrm{NaR}$ absent & 1 & & Odds Ratio (M-H, Fixed, 95\% CI) & Not estimable \\
\hline
\end{tabular}

Fluoroquinolones for treating typhoid and paratyphoid fever (enteric fever) (Review)

Copyright ( 2008 The Cochrane Collaboration. Published by John Wiley \& Sons, Ltd. 


\begin{tabular}{|c|c|c|c|c|}
\hline Outcome or subgroup title & $\begin{array}{l}\text { No. of } \\
\text { studies }\end{array}$ & $\begin{array}{c}\text { No. of } \\
\text { participants }\end{array}$ & Statistical method & Effect size \\
\hline 1 Clinical failure & 3 & 259 & Odds Ratio (M-H, Fixed, 95\% CI) & $5.80[1.87,17.98]$ \\
\hline 1.1 MDR and $\mathrm{NaR}$ present & 1 & 40 & Odds Ratio (M-H, Fixed, 95\% CI) & Not estimable \\
\hline $\begin{array}{l}1.2 \mathrm{MDR} \text { absent and } \mathrm{NaR} \\
\text { not reported }\end{array}$ & 1 & 50 & Odds Ratio (M-H, Fixed, 95\% CI) & $41.48[2.22,774.60]$ \\
\hline $\begin{array}{l}1.3 \mathrm{MDR} \text { and } \mathrm{NaR} \text { not } \\
\text { reported }\end{array}$ & 1 & 169 & Odds Ratio (M-H, Fixed, 95\% CI) & $2.81[0.73,10.79]$ \\
\hline 2 Microbiological failure & 2 & 209 & Odds Ratio (M-H, Fixed, 95\% CI) & $2.81[0.73,10.79]$ \\
\hline 2.1 MDR and $\mathrm{NaR}$ present & 1 & 40 & Odds Ratio (M-H, Fixed, 95\% CI) & Not estimable \\
\hline $\begin{array}{l}2.2 \mathrm{MDR} \text { and } \mathrm{NaR} \text { not } \\
\text { reported }\end{array}$ & 1 & 169 & Odds Ratio (M-H, Fixed, 95\% CI) & $2.81[0.73,10.79]$ \\
\hline 3 Relapse & 2 & 90 & Odds Ratio (M-H, Fixed, 95\% CI) & $0.19[0.01,3.92]$ \\
\hline 3.1 MDR and $\mathrm{NaR}$ present & 1 & 40 & Odds Ratio (M-H, Fixed, 95\% CI) & Not estimable \\
\hline $\begin{array}{l}3.2 \mathrm{MDR} \text { absent and } \mathrm{NaR} \\
\text { not reported }\end{array}$ & 1 & 50 & Odds Ratio (M-H, Fixed, 95\% CI) & $0.19[0.01,3.92]$ \\
\hline 4 Fever clearance time & 2 & & Mean Difference (IV, Fixed, 95\% CI) & Totals not selected \\
\hline 4.1 MDR and $\mathrm{NaR}$ present & 1 & & Mean Difference (IV, Fixed, 95\% CI) & Not estimable \\
\hline $\begin{array}{l}4.2 \mathrm{MDR} \text { and } \mathrm{NaR} \text { not } \\
\text { reported }\end{array}$ & 1 & & Mean Difference (IV, Fixed, 95\% CI) & Not estimable \\
\hline $\begin{array}{l}5 \text { Length of hospital stay (MDR } \\
\text { and NaR present) }\end{array}$ & 1 & & Mean Difference (IV, Fixed, 95\% CI) & Totals not selected \\
\hline 6 Convalescent faecal carriage & 3 & 259 & Odds Ratio (M-H, Fixed, 95\% CI) & $1.03[0.29,3.61]$ \\
\hline 6.1 MDR and $\mathrm{NaR}$ present & 1 & 40 & Odds Ratio (M-H, Fixed, 95\% CI) & Not estimable \\
\hline $\begin{array}{l}\text { 6.2 MDR absent and } \mathrm{NaR} \\
\text { not reported }\end{array}$ & 1 & 50 & Odds Ratio (M-H, Fixed, 95\% CI) & $1.15[0.23,5.78]$ \\
\hline $\begin{array}{l}6.3 \mathrm{MDR} \text { and } \mathrm{NaR} \text { not } \\
\text { reported }\end{array}$ & 1 & 169 & Odds Ratio (M-H, Fixed, 95\% CI) & $0.88[0.12,6.36]$ \\
\hline 7 Adverse events (not serious) & 2 & 209 & Odds Ratio (M-H, Fixed, 95\% CI) & $0.92[0.40,2.15]$ \\
\hline 7.1 MDR and $\mathrm{NaR}$ present & 1 & 40 & Odds Ratio (M-H, Fixed, 95\% CI) & $1.24[0.34,4.46]$ \\
\hline $\begin{array}{l}7.2 \mathrm{MDR} \text { and } \mathrm{NaR} \text { not } \\
\text { reported }\end{array}$ & 1 & 169 & Odds Ratio (M-H, Fixed, 95\% CI) & $0.73[0.24,2.29]$ \\
\hline
\end{tabular}

\section{Comparison 8. Norfloxacin vs ceftriaxone}

\begin{tabular}{|c|c|c|c|c|}
\hline Outcome or subgroup title & $\begin{array}{l}\text { No. of } \\
\text { studies }\end{array}$ & $\begin{array}{c}\text { No. of } \\
\text { participants }\end{array}$ & Statistical method & Effect size \\
\hline $\begin{array}{l}1 \text { Clinical failure (NaR not } \\
\text { reported) }\end{array}$ & 1 & & Odds Ratio (M-H, Fixed, 95\% CI) & Totals not selected \\
\hline 2 Relapse (NaR not reported) & 1 & & Odds Ratio (M-H, Fixed, 95\% CI) & Subtotals only \\
\hline $\begin{array}{l}3 \text { Fever clearance time }(\mathrm{NaR} \text { not } \\
\text { reported) }\end{array}$ & 1 & & Mean Difference (IV, Fixed, 95\% CI) & Subtotals only \\
\hline $\begin{array}{l}4 \text { Adverse events (not serious) } \\
\text { (NaR not reported) }\end{array}$ & 1 & & Odds Ratio (M-H, Fixed, 95\% CI) & Subtotals only \\
\hline
\end{tabular}




\begin{tabular}{|c|c|c|c|c|}
\hline Outcome or subgroup title & $\begin{array}{l}\text { No. of } \\
\text { studies }\end{array}$ & $\begin{array}{c}\text { No. of } \\
\text { participants }\end{array}$ & Statistical method & Effect size \\
\hline 1 Clinical failure & 5 & & Odds Ratio (M-H, Fixed, 95\% CI) & Subtotals only \\
\hline 1.1 Versus pefloxacin & 3 & 200 & Odds Ratio (M-H, Fixed, 95\% CI) & $30.60[5.75,162.86]$ \\
\hline 1.2 Versus ofloxacin & 3 & 123 & Odds Ratio (M-H, Fixed, 95\% CI) & $28.15[4.80,165.14]$ \\
\hline 1.3 Versus enoxacin & 2 & 142 & Odds Ratio (M-H, Fixed, 95\% CI) & $4.15[1.77,9.76]$ \\
\hline 2 Relapse & 2 & & Odds Ratio (M-H, Fixed, 95\% CI) & Subtotals only \\
\hline 2.1 Versus ofloxacin & 2 & 106 & Odds Ratio (M-H, Fixed, 95\% CI) & $2.08[0.18,24.31]$ \\
\hline 3 Fever clearance time & 4 & & Mean Difference (IV, Fixed, 95\% CI) & Subtotals only \\
\hline 3.1 Versus pefloxacin & 2 & 144 & Mean Difference (IV, Fixed, 95\% CI) & $18.83[2.62,35.03]$ \\
\hline 3.2 Versus ofloxacin & 2 & 73 & Mean Difference (IV, Fixed, 95\% CI) & $30.26[10.42,50.10]$ \\
\hline 3.3 Versus enoxacin & 1 & 102 & Mean Difference (IV, Fixed, 95\% CI) & $60.0[33.81,86.19]$ \\
\hline 4 Convalescent faecal carriage & 2 & & Odds Ratio (M-H, Fixed, 95\% CI) & Subtotals only \\
\hline 4.1 Versus pefloxacin & 1 & 56 & Odds Ratio (M-H, Fixed, 95\% CI) & $14.6[0.71,298.42]$ \\
\hline 4.2 Versus ofloxacin & 2 & 106 & Odds Ratio (M-H, Fixed, 95\% CI) & $3.90[0.63,24.30]$ \\
\hline 4.3 Versus enoxacin & 1 & 40 & Odds Ratio (M-H, Fixed, 95\% CI) & $1.59[0.24,10.70]$ \\
\hline 5 Adverse events (not serious) & 2 & & Odds Ratio (M-H, Fixed, 95\% CI) & Totals not selected \\
\hline 5.1 Versus ofloxacin & 1 & & Odds Ratio (M-H, Fixed, 95\% CI) & Not estimable \\
\hline 5.2 Versus enoxacin & 1 & & Odds Ratio (M-H, Fixed, 95\% CI) & Not estimable \\
\hline
\end{tabular}

Comparison 10. Fluoroquinolones for 2 days vs 3 days

\begin{tabular}{|c|c|c|c|c|}
\hline Outcome or subgroup title & $\begin{array}{l}\text { No. of } \\
\text { studies }\end{array}$ & $\begin{array}{c}\text { No. of } \\
\text { participants }\end{array}$ & Statistical method & Effect size \\
\hline 1 Clinical failure ( $\mathrm{NaR}$ present) & 3 & & Odds Ratio (M-H, Fixed, 95\% CI) & Subtotals only \\
\hline 1.1 Adults only & 1 & 100 & Odds Ratio (M-H, Fixed, 95\% CI) & $0.17[0.02,1.47]$ \\
\hline 1.2 Children only & 2 & 296 & Odds Ratio (M-H, Fixed, 95\% CI) & $2.22[0.81,6.12]$ \\
\hline $\begin{array}{l}2 \text { Microbiological failure ( } \mathrm{NaR} \\
\text { present) }\end{array}$ & 2 & & Odds Ratio (M-H, Fixed, 95\% CI) & Subtotals only \\
\hline 2.1 Children only & 2 & 296 & Odds Ratio (M-H, Fixed, 95\% CI) & $1.96[0.42,9.05]$ \\
\hline 3 Relapse (NaR present) & 3 & & Odds Ratio (M-H, Fixed, 95\% CI) & Subtotals only \\
\hline 3.1 Adults only & 1 & 50 & Odds Ratio (M-H, Fixed, 95\% CI) & $0.35[0.01,8.93]$ \\
\hline 3.2 Children only & 2 & 262 & Odds Ratio (M-H, Fixed, 95\% CI) & $2.61[0.38,18.03]$ \\
\hline $\begin{array}{l}4 \text { Fever clearance time }(\mathrm{NaR} \\
\text { present })\end{array}$ & 3 & & Mean Difference (IV, Fixed, 95\% CI) & Subtotals only \\
\hline 4.1 Adults only & 1 & 100 & Mean Difference (IV, Fixed, 95\% CI) & Not estimable \\
\hline 4.2 Children only & 2 & 296 & Mean Difference (IV, Fixed, 95\% CI) & $-8.55[-20.10,3.00]$ \\
\hline $\begin{array}{l}5 \text { Length of hospital stay ( } \mathrm{NaR} \\
\text { present) }\end{array}$ & 3 & & Mean Difference (IV, Fixed, 95\% CI) & Subtotals only \\
\hline 5.1 Adults only & 1 & 100 & Mean Difference (IV, Fixed, 95\% CI) & $-0.20[-0.79,0.39]$ \\
\hline 5.2 Children only & 2 & 296 & Mean Difference (IV, Fixed, 95\% CI) & $-0.44[-0.98,0.09]$ \\
\hline $\begin{array}{l}6 \text { Convalescent faecal carriage } \\
(\mathrm{NaR} \text { present })\end{array}$ & 2 & & Odds Ratio (M-H, Fixed, 95\% CI) & Subtotals only \\
\hline 6.1 Children only & 2 & 262 & Odds Ratio (M-H, Fixed, 95\% CI) & $0.30[0.01,7.75]$ \\
\hline 7 Complications (NaR present) & 3 & & Odds Ratio (M-H, Fixed, 95\% CI) & Subtotals only \\
\hline
\end{tabular}


7.1 Adults only

7.2 Children only

8 Adverse events (not serious)

(NaR present)

8.1 Children only
100

296

2

296
Odds Ratio (M-H, Fixed, 95\% CI)

Odds Ratio (M-H, Fixed, 95\% CI)

Odds Ratio (M-H, Fixed, 95\% CI)

Odds Ratio (M-H, Fixed, 95\% CI)

$0.12[0.01,2.21]$

$1.03[0.21,4.96]$

Subtotals only

$0.29[0.01,7.28]$

Comparison 11. Fluoroquinolones for 3 days vs 5 days

\begin{tabular}{|c|c|c|c|c|}
\hline Outcome or subgroup title & $\begin{array}{l}\text { No. of } \\
\text { studies }\end{array}$ & $\begin{array}{c}\text { No. of } \\
\text { participants }\end{array}$ & Statistical method & Effect size \\
\hline $\begin{array}{l}1 \text { Clinical failure ( } \mathrm{NaR} \text { not } \\
\text { reported) }\end{array}$ & 1 & & Odds Ratio (M-H, Fixed, 95\% CI) & Totals not selected \\
\hline 1.1 Adults only & 1 & & Odds Ratio (M-H, Fixed, 95\% CI) & Not estimable \\
\hline 2 Relapse & 2 & & Odds Ratio (M-H, Fixed, 95\% CI) & Totals not selected \\
\hline $\begin{array}{l}2.1 \text { Adults only (NaR not } \\
\text { reported) }\end{array}$ & 1 & & Odds Ratio (M-H, Fixed, 95\% CI) & Not estimable \\
\hline $\begin{array}{l}2.2 \text { Children mostly (NaR } \\
\text { present) }\end{array}$ & 1 & & Odds Ratio (M-H, Fixed, 95\% CI) & Not estimable \\
\hline 3 Fever clearance time & 2 & & Mean Difference (IV, Fixed, 95\% CI) & Totals not selected \\
\hline $\begin{array}{l}3.1 \text { Adults only }(\mathrm{NaR} \text { not } \\
\text { reported) }\end{array}$ & 1 & & Mean Difference (IV, Fixed, 95\% CI) & Not estimable \\
\hline $\begin{array}{l}3.2 \text { Children mostly }(\mathrm{NaR} \\
\text { present) }\end{array}$ & 1 & & Mean Difference (IV, Fixed, 95\% CI) & Not estimable \\
\hline $\begin{array}{l}4 \text { Length of hospital stay ( } \mathrm{NaR} \text { not } \\
\text { reported) }\end{array}$ & 1 & & Mean Difference (IV, Fixed, 95\% CI) & Totals not selected \\
\hline 4.1 Adults only & 1 & & Mean Difference (IV, Fixed, 95\% CI) & Not estimable \\
\hline $\begin{array}{l}5 \text { Adverse events (not serious) } \\
\quad \text { (NaR present) }\end{array}$ & 1 & & Odds Ratio (M-H, Fixed, 95\% CI) & Totals not selected \\
\hline 5.1 Children mostly & 1 & & Odds Ratio (M-H, Fixed, 95\% CI) & Not estimable \\
\hline
\end{tabular}

\section{Comparison 12. Fluoroquinolones for 5 days vs 7 days}

\begin{tabular}{|c|c|c|c|c|}
\hline Outcome or subgroup title & $\begin{array}{l}\text { No. of } \\
\text { studies }\end{array}$ & $\begin{array}{c}\text { No. of } \\
\text { participants }\end{array}$ & Statistical method & Effect size \\
\hline $\begin{array}{l}1 \text { Microbiological failure ( } \mathrm{NaR} \text { not } \\
\text { reported) }\end{array}$ & 1 & & Odds Ratio (M-H, Fixed, 95\% CI) & Subtotals only \\
\hline 2 Relapse (NaR not reported) & 1 & & Odds Ratio (M-H, Fixed, 95\% CI) & Subtotals only \\
\hline $\begin{array}{l}3 \text { Fever clearance time }(\mathrm{NaR} \text { not } \\
\text { reported) }\end{array}$ & 1 & & Mean Difference (IV, Fixed, 95\% CI) & Subtotals only \\
\hline $\begin{array}{l}4 \text { Adverse events (not serious) } \\
\text { (NaR not reported) }\end{array}$ & 1 & & Odds Ratio (M-H, Fixed, 95\% CI) & Subtotals only \\
\hline
\end{tabular}


Comparison 13. Fluoroquinolones for 7 days vs 10 or 14 days

\begin{tabular}{lcccc} 
Outcome or subgroup title & $\begin{array}{c}\text { No. of } \\
\text { studies }\end{array}$ & $\begin{array}{c}\text { No. of } \\
\text { participants }\end{array}$ & Statistical method & Effect size \\
\hline $\begin{array}{l}1 \text { Microbiological failure (NaR not } \\
\text { reported) }\end{array}$ & 2 & 87 & Odds Ratio (M-H, Fixed, 95\% CI) & $1.39[0.08,23.41]$ \\
2 Relapse $(\mathrm{NaR}$ not reported) & 2 & 87 & Odds Ratio (M-H, Fixed, 95\% CI) & $4.28[0.17,109.61]$ \\
\hline
\end{tabular}

\section{Comparison 14. Fluoroquinolones for 10 days vs 14 days}

\begin{tabular}{|c|c|c|c|c|}
\hline Outcome or subgroup title & $\begin{array}{l}\text { No. of } \\
\text { studies }\end{array}$ & $\begin{array}{c}\text { No. of } \\
\text { participants }\end{array}$ & Statistical method & Effect size \\
\hline 1 Relapse (NaR present) & 1 & & Odds Ratio (M-H, Fixed, 95\% CI) & Subtotals only \\
\hline $\begin{array}{l}2 \text { Fever clearance time }(\mathrm{NaR} \\
\text { present })\end{array}$ & 1 & & Mean Difference (IV, Fixed, 95\% CI) & Subtotals only \\
\hline $\begin{array}{l}3 \text { Adverse events (not serious) } \\
\quad(\mathrm{NaR} \text { present })\end{array}$ & 1 & & Odds Ratio (M-H, Fixed, 95\% CI) & Subtotals only \\
\hline
\end{tabular}

\section{Analysis I.I. Comparison I Fluoroquinolones vs chloramphenicol, Outcome I Clinical failure.}

Review: Fluoroquinolones for treating typhoid and paratyphoid fever (enteric fever)

Comparison: | Fluoroquinolones vs chloramphenicol

Outcome: I Clinical failure

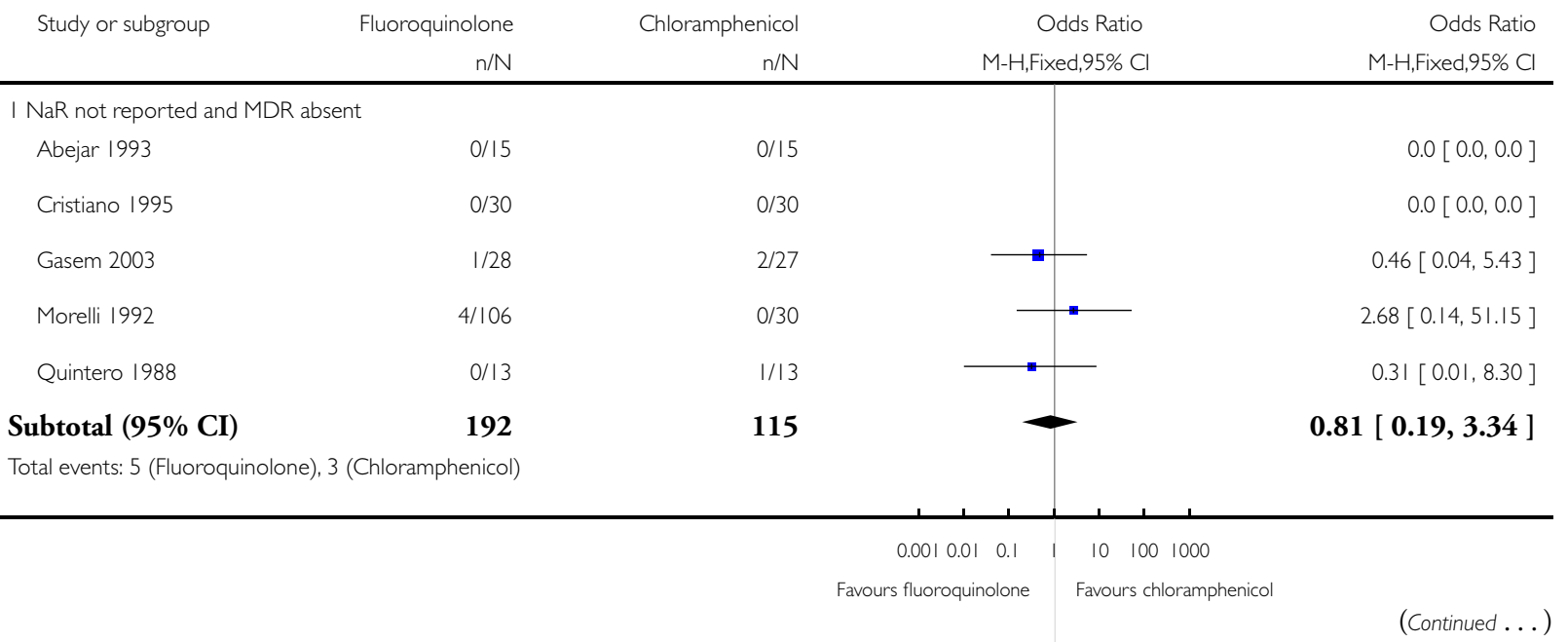

Fluoroquinolones for treating typhoid and paratyphoid fever (enteric fever) (Review)

Copyright ( 2008 The Cochrane Collaboration. Published by John Wiley \& Sons, Ltd. 


\begin{tabular}{|c|c|c|c|c|}
\hline Study or subgroup & $\begin{array}{r}\text { Fluoroquinolone } \\
\mathrm{n} / \mathrm{N}\end{array}$ & $\begin{array}{r}\text { Chloramphenicol } \\
n / N\end{array}$ & $\begin{array}{c}\text { Odds Ratio } \\
\text { M-H,Fixed,95\% Cl }\end{array}$ & $\begin{array}{c}\text { (... Continued) } \\
\text { Odds Ratio } \\
\text { M-H,Fixed,95\% Cl }\end{array}$ \\
\hline \multicolumn{5}{|c|}{ Heterogeneity: $\mathrm{Ch}^{2}=1.16, \mathrm{df}=2(\mathrm{P}=0.56) ; \mathrm{I}^{2}=0.0 \%$} \\
\hline \multicolumn{5}{|c|}{ Test for overall effect: $Z=0.30(P=0.77)$} \\
\hline \multicolumn{5}{|c|}{$2 \mathrm{NaR}$ absent and MDR present } \\
\hline Phongmany 2005 & 0/27 & $1 / 23$ & \begin{tabular}{l|l} 
\\
$*$
\end{tabular} & $0.27[0.01,7.02]$ \\
\hline Subtotal $(95 \% \mathrm{CI})$ & 27 & 23 & - & $0.27[0.01,7.02]$ \\
\hline \multicolumn{5}{|c|}{ Total events: 0 (Fluoroquinolone), I (Chloramphenicol) } \\
\hline \multicolumn{5}{|c|}{ Heterogeneity: not applicable } \\
\hline \multicolumn{5}{|c|}{ Test for overall effect: $Z=0.78(P=0.43)$} \\
\hline \multicolumn{5}{|c|}{$3 \mathrm{NaR}$ and MDR not reported } \\
\hline Arnold 1993 & $0 / 57$ & 1/34 & - & $0.19[0.01,4.90]$ \\
\hline Gottuzzo 1992 & 1/48 & $0 / 48$ & & $3.06[0.12,77.09]$ \\
\hline Yousaf 1992 & $1 / 25$ & $2 / 25$ & $\rightarrow-$ & $0.48[0.04,5.65]$ \\
\hline Subtotal $(95 \% \mathrm{CI})$ & 130 & 107 & r & $0.65[0.14,2.91]$ \\
\hline \multicolumn{5}{|c|}{ Total events: 2 (Fluoroquinolone), 3 (Chloramphenicol) } \\
\hline \multicolumn{5}{|c|}{ Heterogeneity: $\mathrm{Ch}^{2}=1.48, \mathrm{df}=2(\mathrm{P}=0.48) ; \mathrm{I}^{2}=0.0 \%$} \\
\hline \multicolumn{5}{|c|}{ Test for overall effect: $Z=0.56(P=0.57)$} \\
\hline Total $(95 \% \mathrm{CI})$ & 349 & 245 & 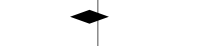 & $0.65[0.25,1.72]$ \\
\hline \multicolumn{5}{|c|}{ Total events: 7 (Fluoroquinolone), 7 (Chloramphenicol) } \\
\hline \multicolumn{5}{|c|}{ Heterogeneity: $\mathrm{Chi}^{2}=2.92, \mathrm{df}=6(\mathrm{P}=0.82) ; \mathrm{I}^{2}=0.0 \%$} \\
\hline Test for overall effect: $Z=$ & 0.39) & & & \\
\hline
\end{tabular}




\section{Analysis I.2. Comparison I Fluoroquinolones vs chloramphenicol, Outcome 2 Microbiological failure.}

\begin{tabular}{|c|c|c|c|c|}
\hline \multicolumn{5}{|c|}{ Comparison: I Fluoroquinolones vs chloramphenicol } \\
\hline \multicolumn{5}{|c|}{ Outcome: 2 Microbiological failure } \\
\hline \multirow[t]{2}{*}{ Study or subgroup } & Fluoroquinolone & Chloramphenicol & Odds Ratio & Odds Ratio \\
\hline & $\mathrm{n} / \mathrm{N}$ & $\mathrm{n} / \mathrm{N}$ & M-H,Fixed,95\% Cl & M-H,Fixed,95\% Cl \\
\hline \multicolumn{5}{|c|}{ I NaR not reported and MDR absent } \\
\hline Abejar 1993 & $0 / 15$ & $1 / 15$ & $\longrightarrow \cdot$ & $0.31[0.01,8.28]$ \\
\hline Bran 1991 & $0 / 51$ & $0 / 51$ & & $0.0[0.0,0.0]$ \\
\hline Cristiano 1995 & $0 / 30$ & $0 / 30$ & & $0.0[0.0,0.0]$ \\
\hline Gasem 2003 & $7 / 23$ & $8 / 22$ & $\rightarrow$ & $0.77[0.22,2.65]$ \\
\hline Subtotal $(95 \% \mathrm{CI})$ & 119 & 118 & 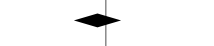 & $0.67[0.21,2.12]$ \\
\hline \multicolumn{5}{|c|}{ Total events: 7 (Fluoroquinolone), 9 (Chloramphenicol) } \\
\hline \multicolumn{5}{|c|}{ Heterogeneity: $\mathrm{Ch}^{2}=0.25, \mathrm{df}=\mathrm{I}(\mathrm{P}=0.62) ; \mathrm{I}^{2}=0.0 \%$} \\
\hline \multicolumn{5}{|c|}{ Test for overall effect: $Z=0.67(P=0.50)$} \\
\hline \multicolumn{5}{|c|}{$2 \mathrm{NaR}$ and MDR not reported } \\
\hline Arnold 1993 & $2 / 57$ & $5 / 34$ & $\longrightarrow$ & $0.21[0.04,1.15]$ \\
\hline Yousaf 1992 & $1 / 25$ & $3 / 25$ & $\longrightarrow$ & $0.31[0.03,3.16]$ \\
\hline Subtotal $(95 \% \mathrm{CI})$ & 82 & 59 & $\longrightarrow$ & $0.24[0.06,0.96]$ \\
\hline \multicolumn{5}{|c|}{ Total events: 3 (Fluoroquinolone), 8 (Chloramphenicol) } \\
\hline \multicolumn{5}{|c|}{ Heterogeneity: $\mathrm{Chi}^{2}=0.06, \mathrm{df}=\mid(P=0.80) ; 1^{2}=0.0 \%$} \\
\hline \multicolumn{5}{|c|}{ Test for overall effect: $Z=2.02(P=0.043)$} \\
\hline Total $(95 \% \mathrm{CI})$ & 201 & 177 & - & $0.43[0.18,1.03]$ \\
\hline \multicolumn{5}{|c|}{ Total events: 10 (Fluoroquinolone), 17 (Chloramphenicol) } \\
\hline \multicolumn{5}{|c|}{ Heterogeneity: $\mathrm{Chi}^{2}=1.62, \mathrm{df}=3(\mathrm{P}=0.65) ; \mathrm{I}^{2}=0.0 \%$} \\
\hline Test for overall effect: $Z$ = & $=0.058)$ & & & \\
\hline
\end{tabular}




\section{Analysis I.3. Comparison I Fluoroquinolones vs chloramphenicol, Outcome 3 Relapse.}

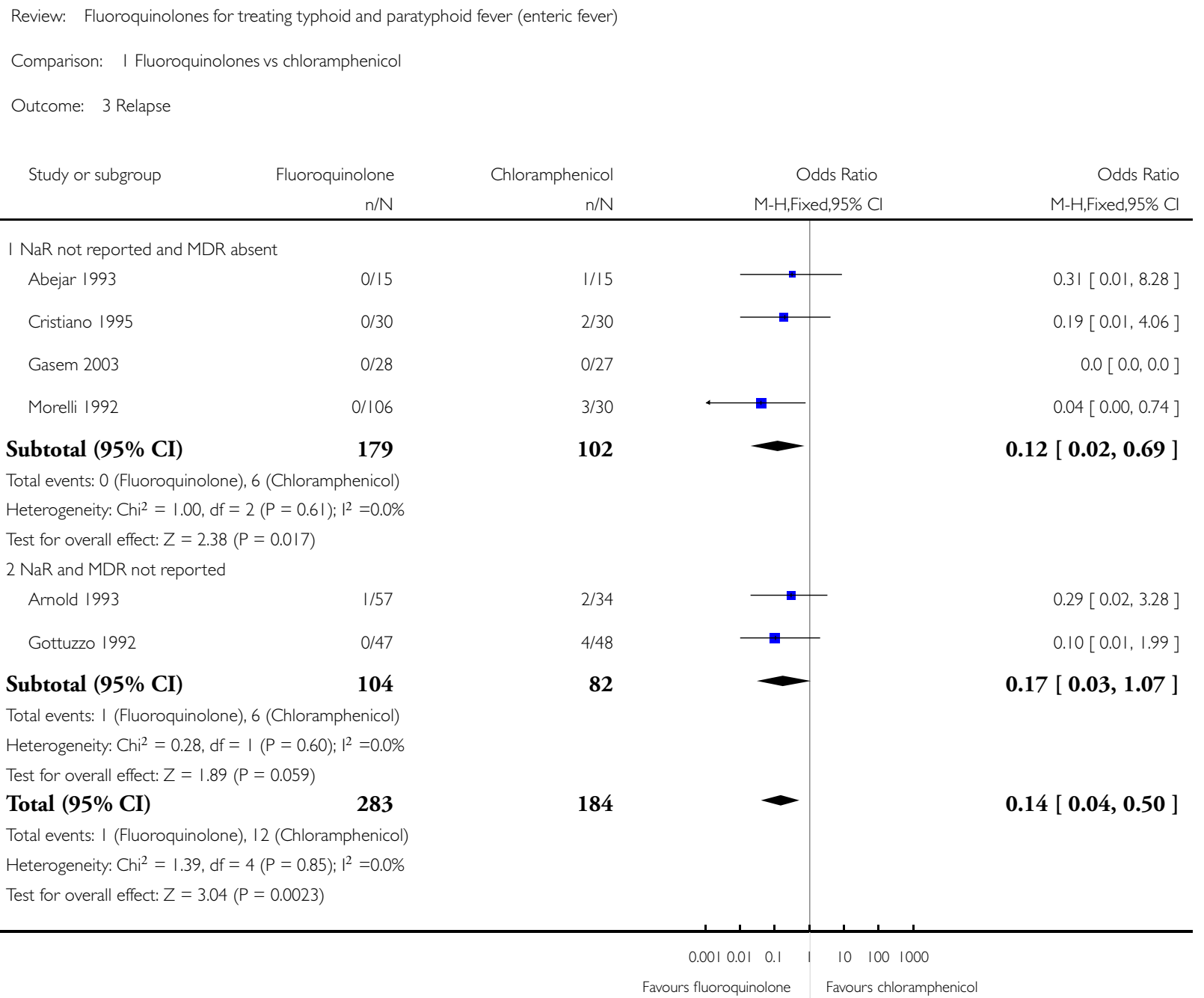




\section{Analysis I.4. Comparison I Fluoroquinolones vs chloramphenicol, Outcome 4 Fever clearance time.}

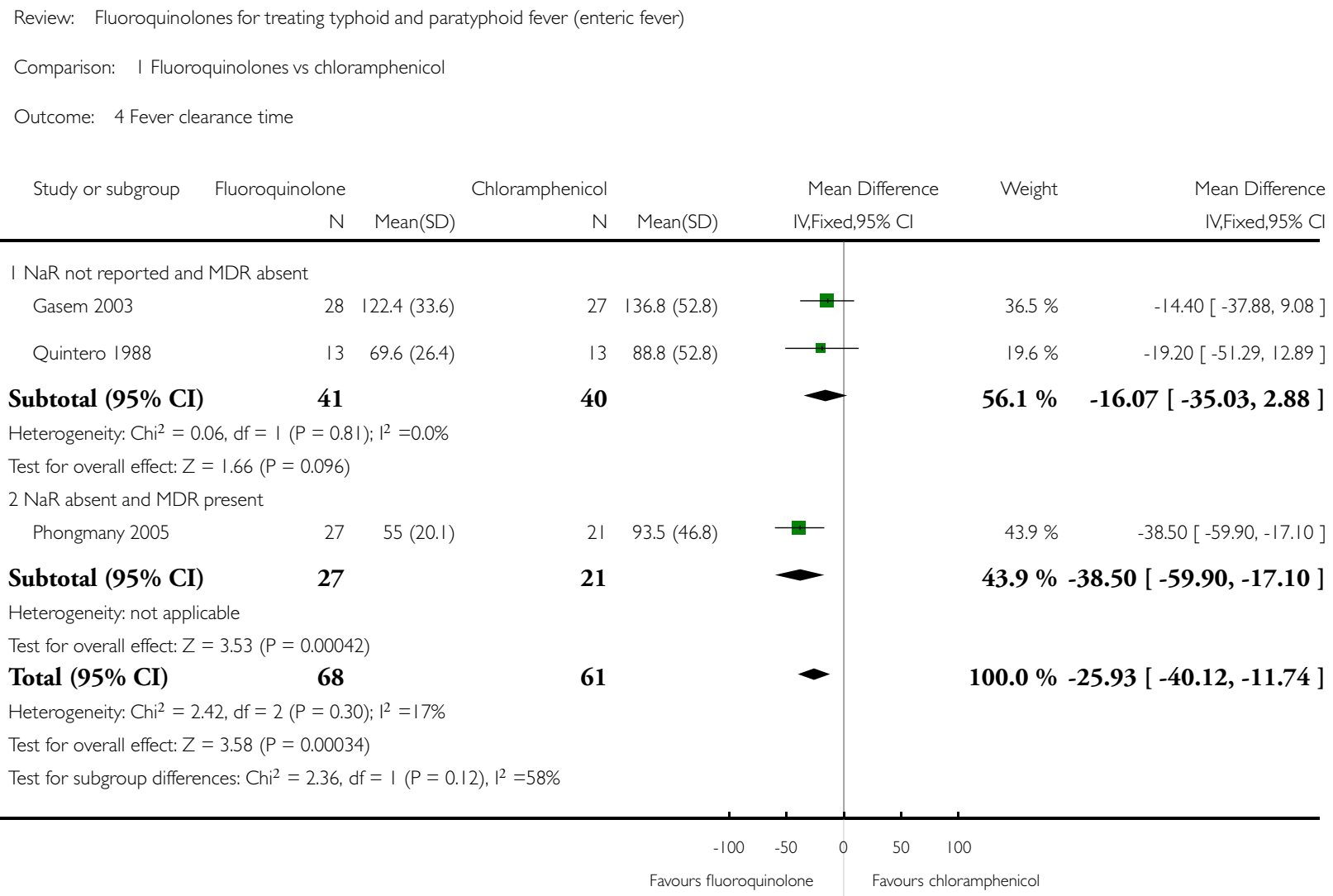




\section{Analysis I.5. Comparison I Fluoroquinolones vs chloramphenicol, Outcome 5 Length of hospital stay.}

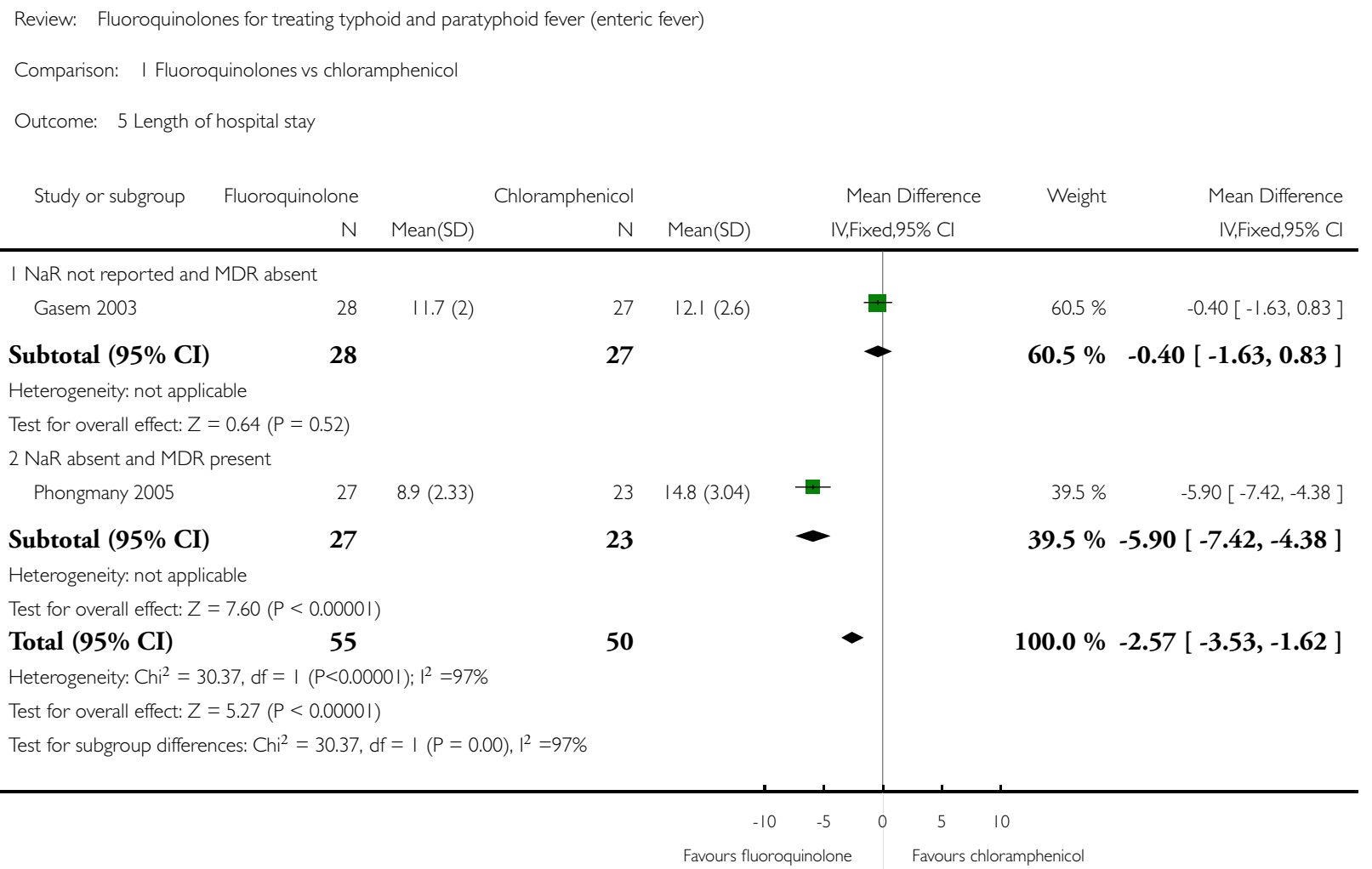


Analysis I.6. Comparison I Fluoroquinolones vs chloramphenicol, Outcome 6 Convalescent faecal carriage (NaR not reported and MDR absent).

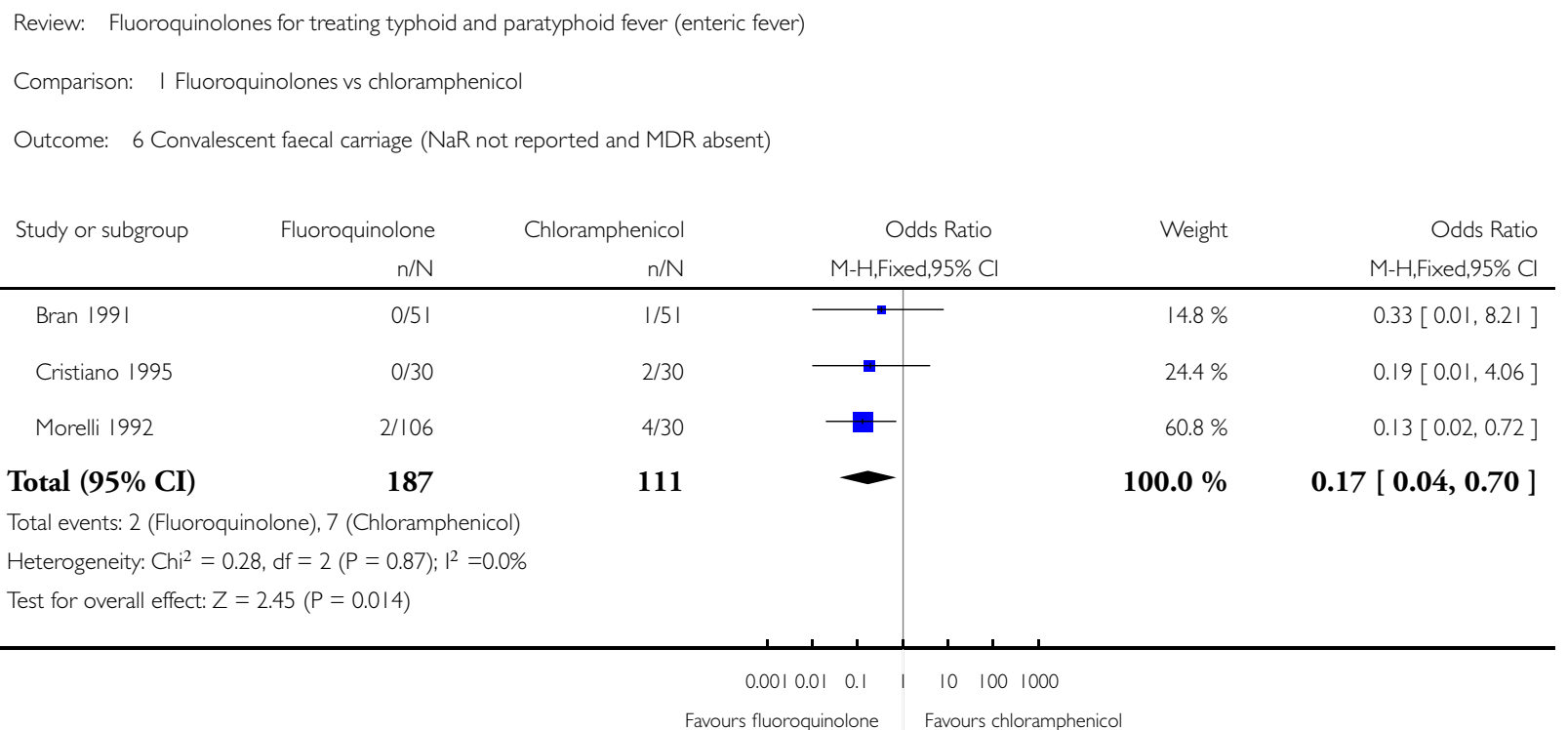

\section{Analysis I.7. Comparison I Fluoroquinolones vs chloramphenicol, Outcome 7 Complications.}

Review: Fluoroquinolones for treating typhoid and paratyphoid fever (enteric fever)

Comparison: I Fluoroquinolones vs chloramphenicol

Outcome: 7 Complications

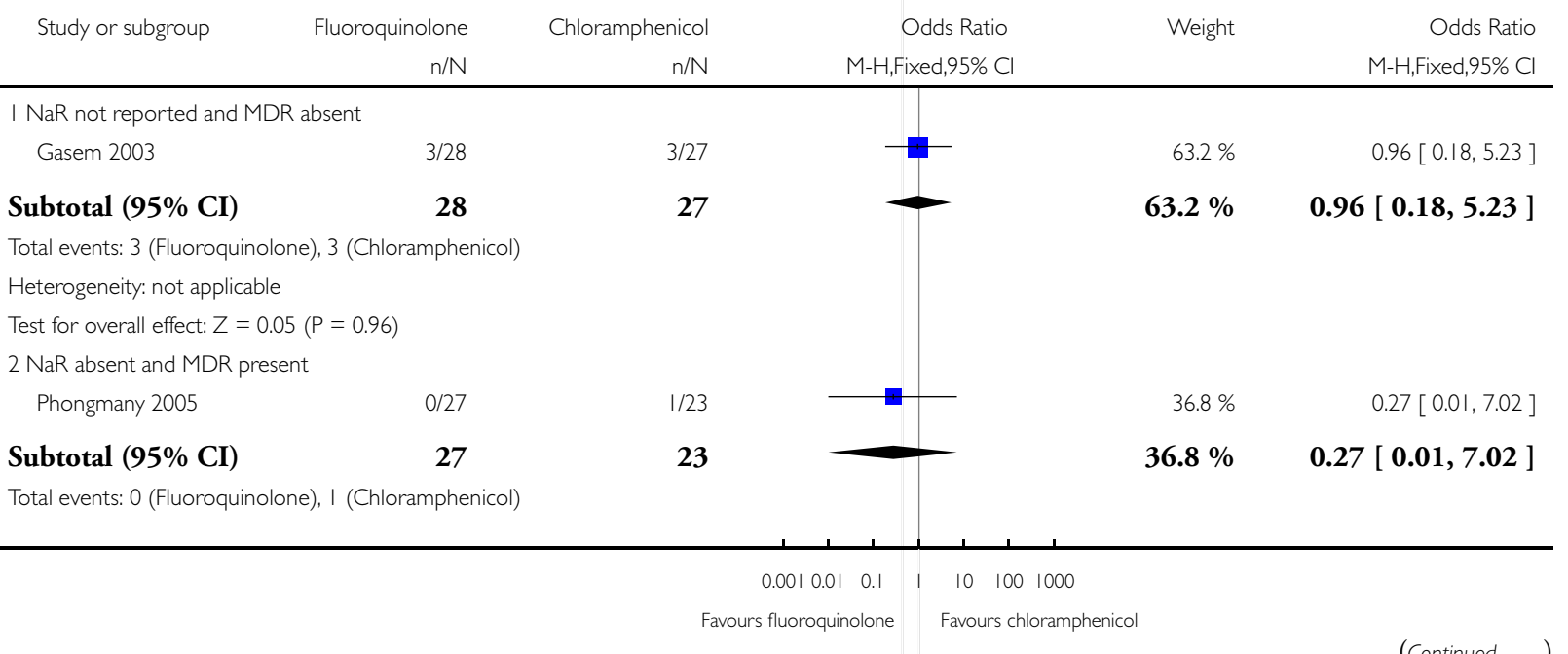

Fluoroquinolones for treating typhoid and paratyphoid fever (enteric fever) (Review)

Copyright ( 2008 The Cochrane Collaboration. Published by John Wiley \& Sons, Ltd. 


M. H, Fixed, $95 \%$ Cl

Total events: 3 (Fluoroquinolone), 4 (Chloramphenicol)

Heterogeneity: $\mathrm{Chi}^{2}=0.46, \mathrm{df}=\mathrm{I}(\mathrm{P}=0.50) ; \mathrm{I}^{2}=0.0 \%$

Test for overall effect: $Z=0.46(P=0.64)$

$0.0010 .01 \quad 0.1 \quad 1 \quad 10 \quad 100 \quad 1000$

Favours fluoroquinolone Favours chloramphenicol

\section{Analysis I.8. Comparison I Fluoroquinolones vs chloramphenicol, Outcome 8 Adverse events (not serious).}

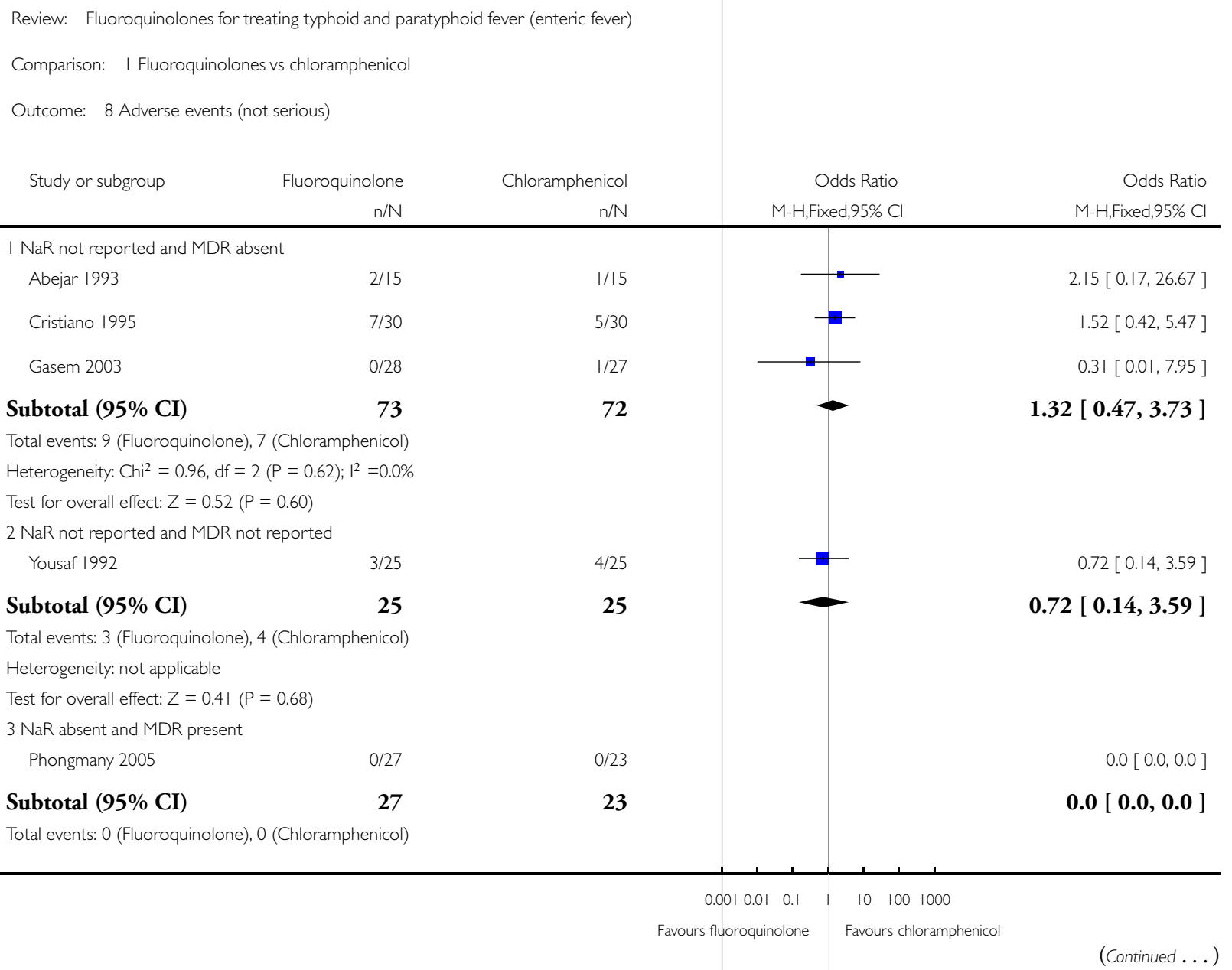




\begin{tabular}{|c|c|c|c|c|}
\hline Study or subgroup & $\begin{array}{r}\text { Fluoroquinolone } \\
\mathrm{n} / \mathrm{N}\end{array}$ & $\begin{array}{r}\text { Chloramphenicol } \\
n / N\end{array}$ & $\begin{array}{c}\text { Odds Ratio } \\
\text { M-H,Fixed,95\% Cl }\end{array}$ & $\begin{array}{c}\text { (... Continued) } \\
\text { Odds Ratio }\end{array}$ \\
\hline \multicolumn{5}{|c|}{ Heterogeneity: not applicable } \\
\hline \multicolumn{5}{|c|}{ Test for overall effect: $Z=0.0(P<0.0000 \mathrm{I})$} \\
\hline Total $(95 \% \mathrm{CI})$ & 125 & 120 & - & $1.10[0.46,2.62]$ \\
\hline \multicolumn{5}{|c|}{ Total events: I2 (Fluoroquinolone), I I (Chloramphenicol) } \\
\hline \multicolumn{5}{|c|}{ Heterogeneity: $\mathrm{Chi}^{2}=1.38, \mathrm{df}=3(\mathrm{P}=0.7 \mathrm{I}) ; \mathrm{I}^{2}=0.0 \%$} \\
\hline \multicolumn{5}{|c|}{ Test for overall effect: $Z=0.21(P=0.83)$} \\
\hline
\end{tabular}

\section{Analysis 2.I. Comparison 2 Fluoroquinolones vs amoxicillin (AMX) or ampicillin (AMP), Outcome I Clinical failure (MDR and NaR not reported).}

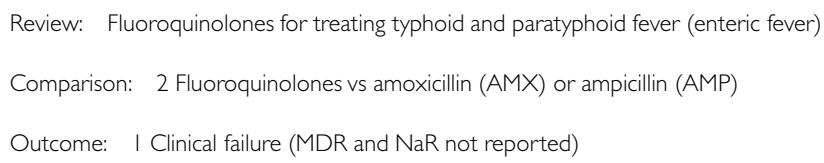

\begin{tabular}{|c|c|c|c|c|c|}
\hline \multirow[t]{2}{*}{ Study or subgroup } & Fluoroquinolone & AMX or AMP & Odds Ratio & Weight & Odds Ratio \\
\hline & $\mathrm{n} / \mathrm{N}$ & $\mathrm{n} / \mathrm{N}$ & M-H,Fixed,95\% Cl & & M-H,Fixed,95\% Cl \\
\hline Flores 1994 & $0 / 20$ & $5 / 20$ & - & $41.1 \%$ & $0.07[0.00,1.34]$ \\
\hline Yousaf 1992 & $1 / 25$ & $8 / 25$ & 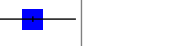 & $58.9 \%$ & $0.09[0.01,0.78]$ \\
\hline Total (95\% CI) & 45 & 45 & - & $100.0 \%$ & $0.08[0.01,0.46]$ \\
\hline \multicolumn{6}{|c|}{ Total events: I (Fluoroquinolone), I3 (AMX or AMP) } \\
\hline \multicolumn{6}{|c|}{ Heterogeneity: $\mathrm{Chi}^{2}=0.02, \mathrm{df}=\mathrm{I}(\mathrm{P}=0.89) ; \mathrm{I}^{2}=0.0 \%$} \\
\hline \multicolumn{6}{|c|}{ Test for overall effect: $Z=2.82(P=0.0048)$} \\
\hline
\end{tabular}

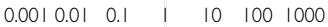
Favours fluoroquinolone Favours AMX or AMP 
Analysis 2.2. Comparison 2 Fluoroquinolones vs amoxicillin (AMX) or ampicillin (AMP), Outcome 2 Microbiological failure (MDR and NaR not reported).

\begin{tabular}{|c|c|c|c|c|c|}
\hline \multicolumn{6}{|c|}{ Comparison: 2 Fluoroquinolones vs amoxicillin (AMX) or ampicillin (AMP) } \\
\hline \multicolumn{6}{|c|}{ Outcome: 2 Microbiological failure (MDR and $\mathrm{NaR}$ not reported) } \\
\hline \multirow[t]{2}{*}{ Study or subgroup } & Fluoroquinolone & AMX or AMP & Odds Ratio & Weight & Odds Ratio \\
\hline & $\mathrm{n} / \mathrm{N}$ & $\mathrm{n} / \mathrm{N}$ & M-H,Fixed,95\% Cl & & M-H,Fixed,95\% Cl \\
\hline Flores 1994 & $0 / 20$ & $3 / 20$ & $\longrightarrow$ & $30.8 \%$ & $0.12[0.01,2.53]$ \\
\hline Yousaf 1992 & $1 / 25$ & $8 / 25$ & & $69.2 \%$ & $0.09[0.01,0.78]$ \\
\hline Total $(95 \% \mathrm{CI})$ & 45 & 45 & & $100.0 \%$ & $0.10[0.02,0.58]$ \\
\hline \multicolumn{6}{|c|}{ Total events: I (Fluoroquinolone), I I (AMX or AMP) } \\
\hline \multicolumn{6}{|c|}{ Heterogeneity: $\mathrm{Chi}^{2}=0.03, \mathrm{df}=\mathrm{I}(\mathrm{P}=0.87) ; \mathrm{I}^{2}=0.0 \%$} \\
\hline \multicolumn{6}{|c|}{ Test for overall effect: $Z=2.57(P=0.010)$} \\
\hline
\end{tabular}

\section{Analysis 2.3. Comparison 2 Fluoroquinolones vs amoxicillin (AMX) or ampicillin (AMP), Outcome 3 Adverse events (not serious) (MDR and NaR not reported).}

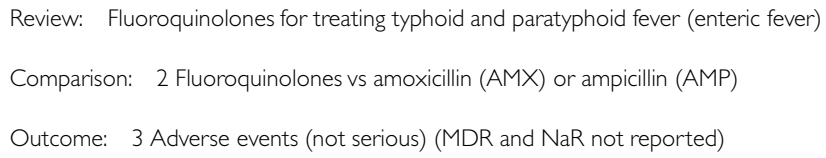

\begin{tabular}{|c|c|c|c|c|}
\hline & $\mathrm{n} / \mathrm{N}$ & $\mathrm{n} / \mathrm{N}$ & M-H,Fixed,95\% Cl & M-H,Fixed,95\% Cl \\
\hline Yousaf 1992 & $3 / 25$ & $11 / 25$ & ए & $0.17[0.04,0.73]$ \\
\hline
\end{tabular}

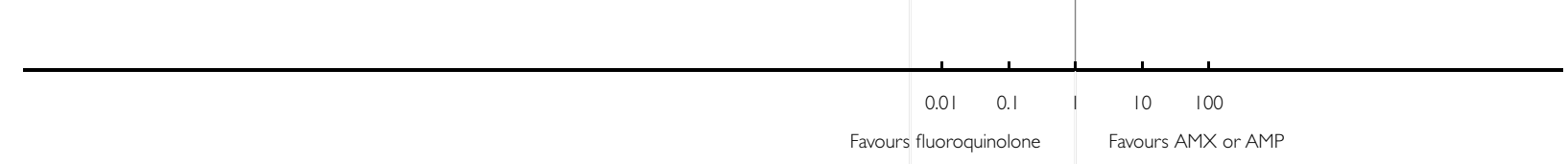




\section{Analysis 3.I. Comparison 3 Fluoroquinolones vs co-trimoxazole, Outcome I Clinical failure (MDR absent).}

\begin{tabular}{|c|c|c|c|c|}
\hline \multicolumn{5}{|c|}{ Comparison: 3 Fluoroquinolones vs co-trimoxazole } \\
\hline \multicolumn{5}{|c|}{ Outcome: I Clinical failure (MDR absent) } \\
\hline \multirow[t]{2}{*}{ Study or subgroup } & Fluoroquinolone & Co-trimoxazole & \multirow{2}{*}{$\begin{array}{c}\text { Odds Ratio } \\
\text { M-H,Fixed,95\% Cl }\end{array}$} & Odds Ratio \\
\hline & $\mathrm{n} / \mathrm{N}$ & $\mathrm{n} / \mathrm{N}$ & & M-H,Fixed,95\% Cl \\
\hline \multicolumn{5}{|l|}{ I NaR absent } \\
\hline Hajji 1988 & $0 / 24$ & $0 / 18$ & & $0.0[0.0,0.0]$ \\
\hline Subtotal $(95 \% \mathrm{CI})$ & 24 & 18 & & $0.0[0.0,0.0]$ \\
\hline \multicolumn{5}{|c|}{ Total events: 0 (Fluoroquinolone), 0 (Co-trimoxazole) } \\
\hline \multicolumn{5}{|c|}{ Heterogeneity: not applicable } \\
\hline \multicolumn{5}{|c|}{ Test for overall effect: $Z=0.0(P<0.0000 \mathrm{I})$} \\
\hline \multicolumn{5}{|c|}{$2 \mathrm{NaR}$ not reported } \\
\hline Limson 1989 & 0/20 & 2/20 & 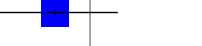 & $0.18[0.01,4.01]$ \\
\hline Subtotal $(95 \% \mathrm{CI})$ & 20 & 20 & & $0.18[0.01,4.01]$ \\
\hline \multicolumn{5}{|c|}{ Total events: 0 (Fluoroquinolone), 2 (Co-trimoxazole) } \\
\hline \multicolumn{5}{|c|}{ Heterogeneity: not applicable } \\
\hline \multicolumn{5}{|c|}{ Test for overall effect: $Z=1.08(P=0.28)$} \\
\hline Total $(95 \% \mathrm{CI})$ & 44 & 38 & & $0.18[0.01,4.01]$ \\
\hline \multicolumn{5}{|c|}{ Total events: 0 (Fluoroquinolone), 2 (Co-trimoxazole) } \\
\hline \multicolumn{5}{|c|}{ Heterogeneity: $C$ Chi ${ }^{2}=0.0, d f=0(P=1.00) ; 1^{2}=0.0 \%$} \\
\hline Test for overall effect: $Z=$ & $=0.28)$ & & & \\
\hline
\end{tabular}


Analysis 3.2. Comparison 3 Fluoroquinolones vs co-trimoxazole, Outcome 2 Microbiological failure (MDR absent).

\begin{tabular}{|c|c|c|c|c|}
\hline \multicolumn{5}{|c|}{ Comparison: 3 Fluoroquinolones vs co-trimoxazole } \\
\hline \multicolumn{5}{|c|}{ Outcome: 2 Microbiological failure (MDR absent) } \\
\hline \multirow[t]{2}{*}{ Study or subgroup } & Fluoroquinolone & Co-trimoxazole & Odds Ratio & Odds Ratio \\
\hline & $\mathrm{n} / \mathrm{N}$ & $\mathrm{n} / \mathrm{N}$ & M-H,Fixed,95\% Cl & M-H,Fixed,95\% Cl \\
\hline \multicolumn{5}{|l|}{ I NaR absent } \\
\hline Hajji 1988 & $0 / 24$ & $0 / 18$ & & $0.0[0.0,0.0]$ \\
\hline Subtotal $(95 \% \mathrm{CI})$ & 24 & 18 & & $0.0[0.0,0.0]$ \\
\hline \multicolumn{5}{|c|}{ Total events: 0 (Fluoroquinolone), 0 (Co-trimoxazole) } \\
\hline \multicolumn{5}{|c|}{ Heterogeneity: not applicable } \\
\hline \multicolumn{5}{|c|}{ Test for overall effect: $Z=0.0(P<0.0000 \mathrm{I})$} \\
\hline \multicolumn{5}{|l|}{$2 \mathrm{NaR}$ not reported } \\
\hline Limson 1989 & 0/20 & 2/20 & & $0.18[0.01,4.01]$ \\
\hline Subtotal $(95 \% \mathrm{CI})$ & 20 & 20 & & $0.18[0.01,4.01]$ \\
\hline \multicolumn{5}{|c|}{ Total events: 0 (Fluoroquinolone), 2 (Co-trimoxazole) } \\
\hline \multicolumn{5}{|c|}{ Heterogeneity: not applicable } \\
\hline \multicolumn{5}{|c|}{ Test for overall effect: $Z=1.08(P=0.28)$} \\
\hline Total $(95 \% \mathrm{CI})$ & 44 & 38 & & $0.18[0.01,4.01]$ \\
\hline \multicolumn{5}{|c|}{ Total events: 0 (Fluoroquinolone), 2 (Co-trimoxazole) } \\
\hline \multicolumn{5}{|c|}{ Heterogeneity: $C$ hi ${ }^{2}=0.0, d f=0(P=1.00) ; 1^{2}=0.0 \%$} \\
\hline Test for overall effect: $Z=$ & $0.28)$ & & & \\
\hline
\end{tabular}


Analysis 3.3. Comparison 3 Fluoroquinolones vs co-trimoxazole, Outcome 3 Adverse events (not serious) (MDR absent).

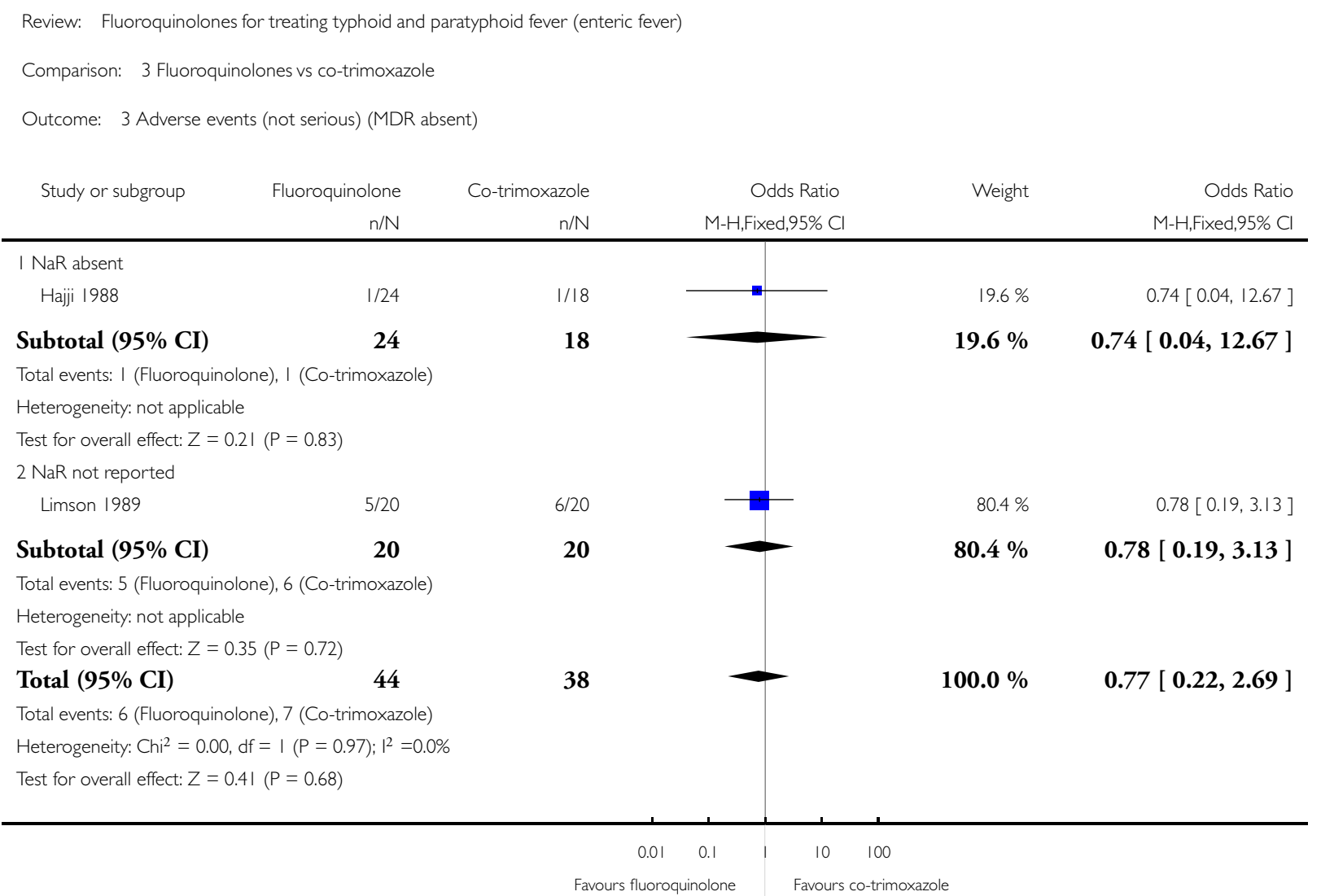




\section{Analysis 4.I. Comparison 4 Fluoroquinolones vs azithromycin, Outcome I Clinical failure (in adults).}

Review: Fluoroquinolones for treating typhoid and paratyphoid fever (enteric fever)

Comparison: 4 Fluoroquinolones vs azithromycin

Outcome: I Clinical failure (in adults)

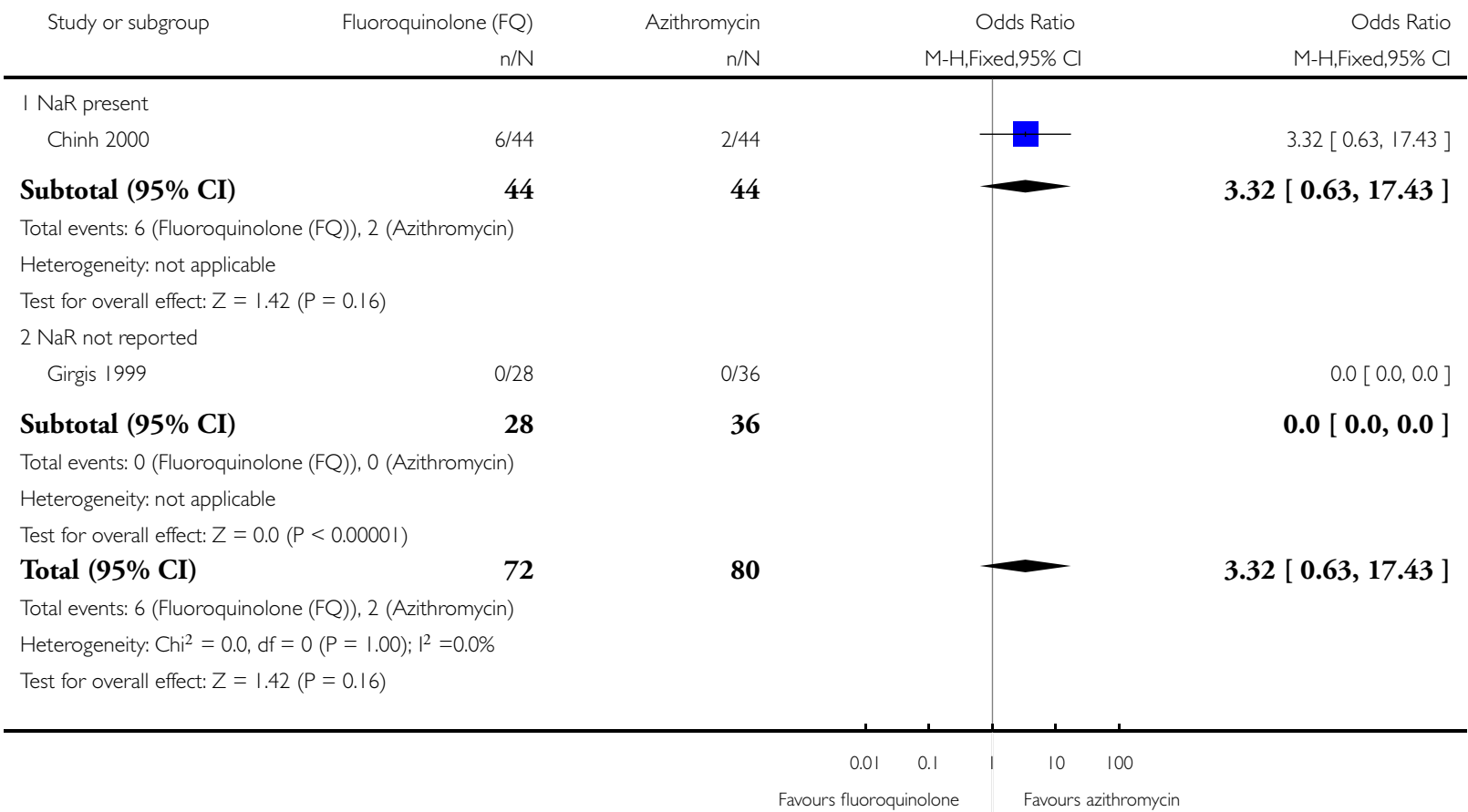


Analysis 4.2. Comparison 4 Fluoroquinolones vs azithromycin, Outcome 2 Microbiological failure (in adults).

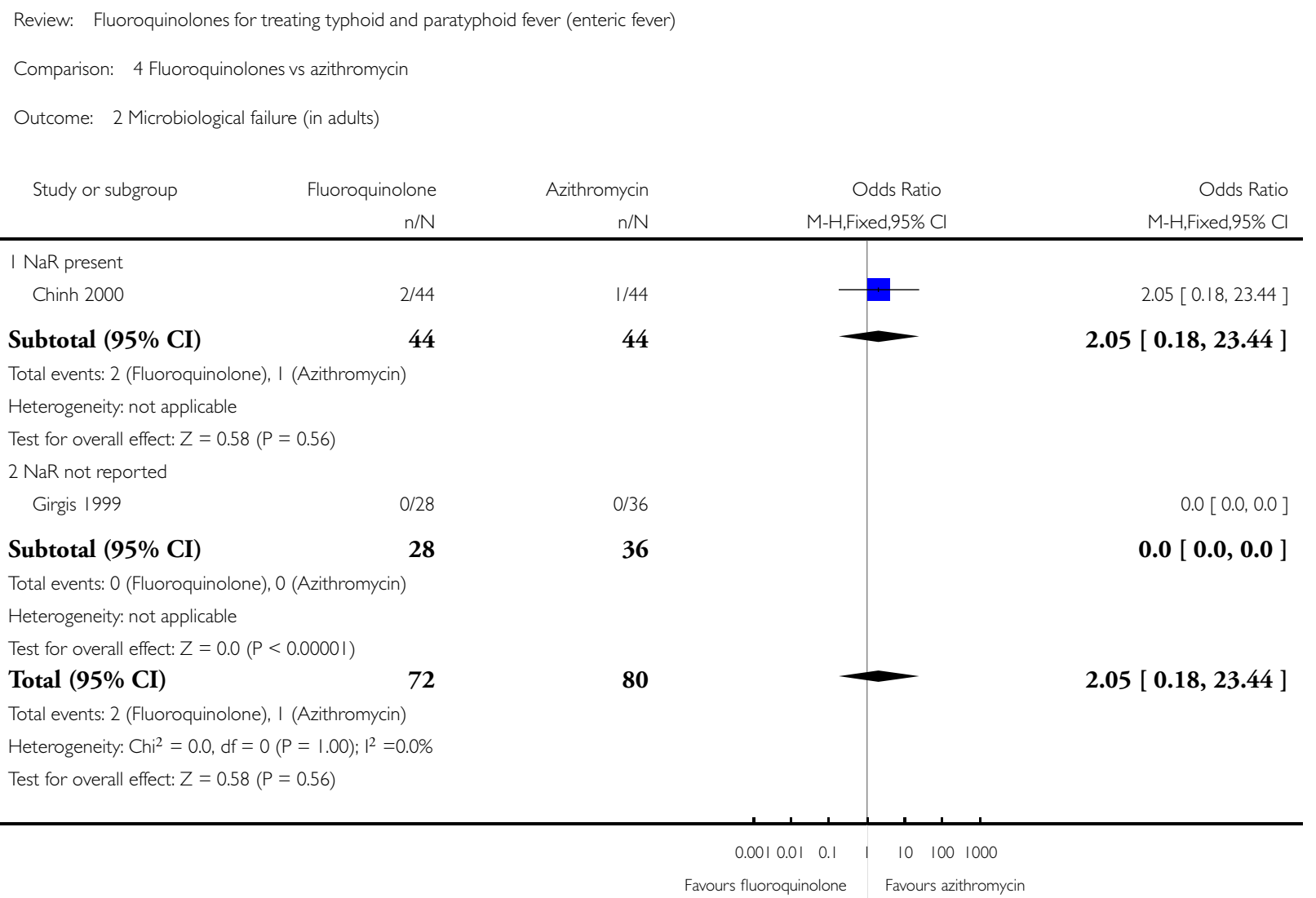




\section{Analysis 4.3. Comparison 4 Fluoroquinolones vs azithromycin, Outcome 3 Relapse (in adults).}

Review: Fluoroquinolones for treating typhoid and paratyphoid fever (enteric fever)

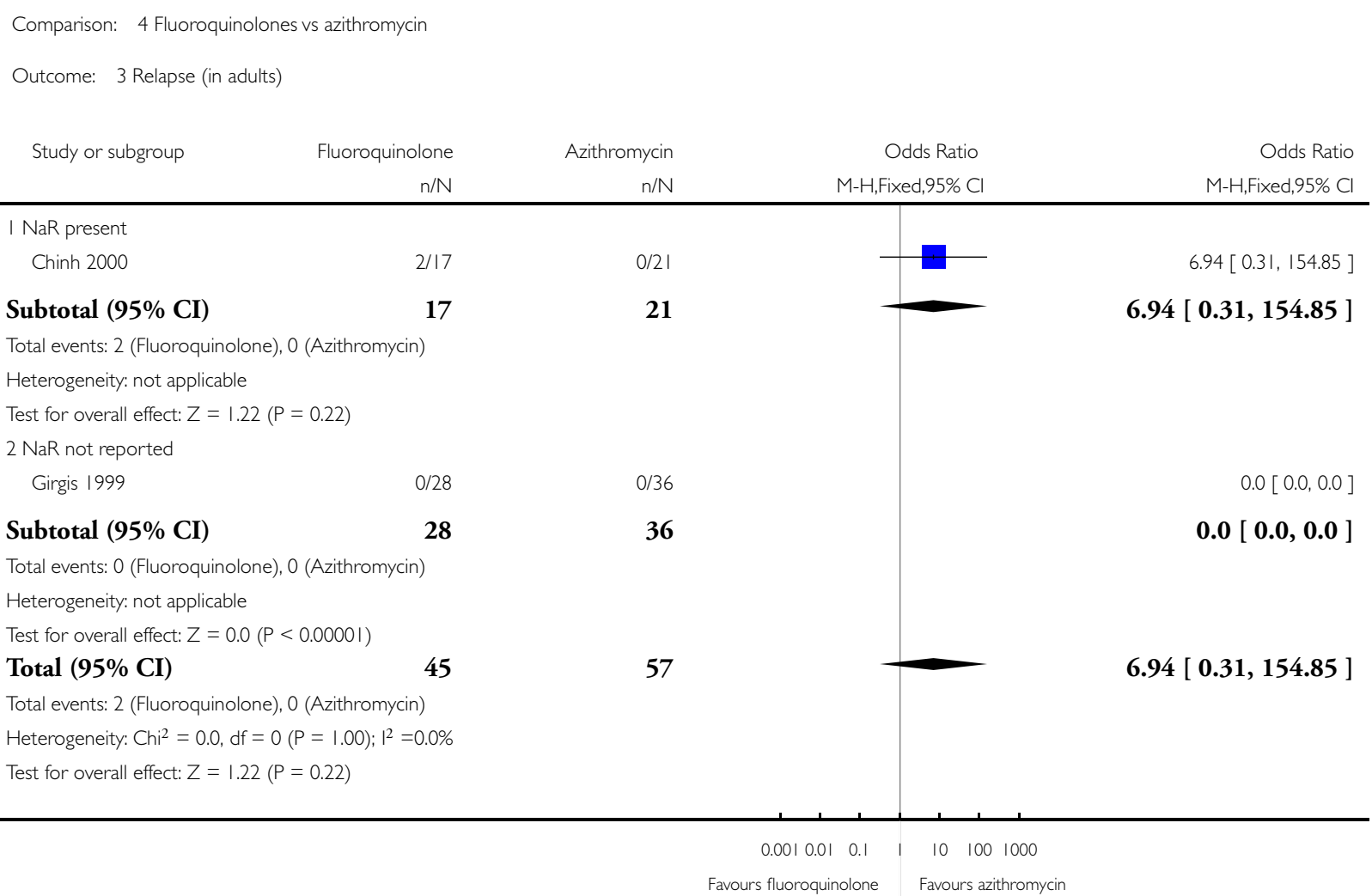


Analysis 4.4. Comparison 4 Fluoroquinolones vs azithromycin, Outcome 4 Fever clearance time (in adults).

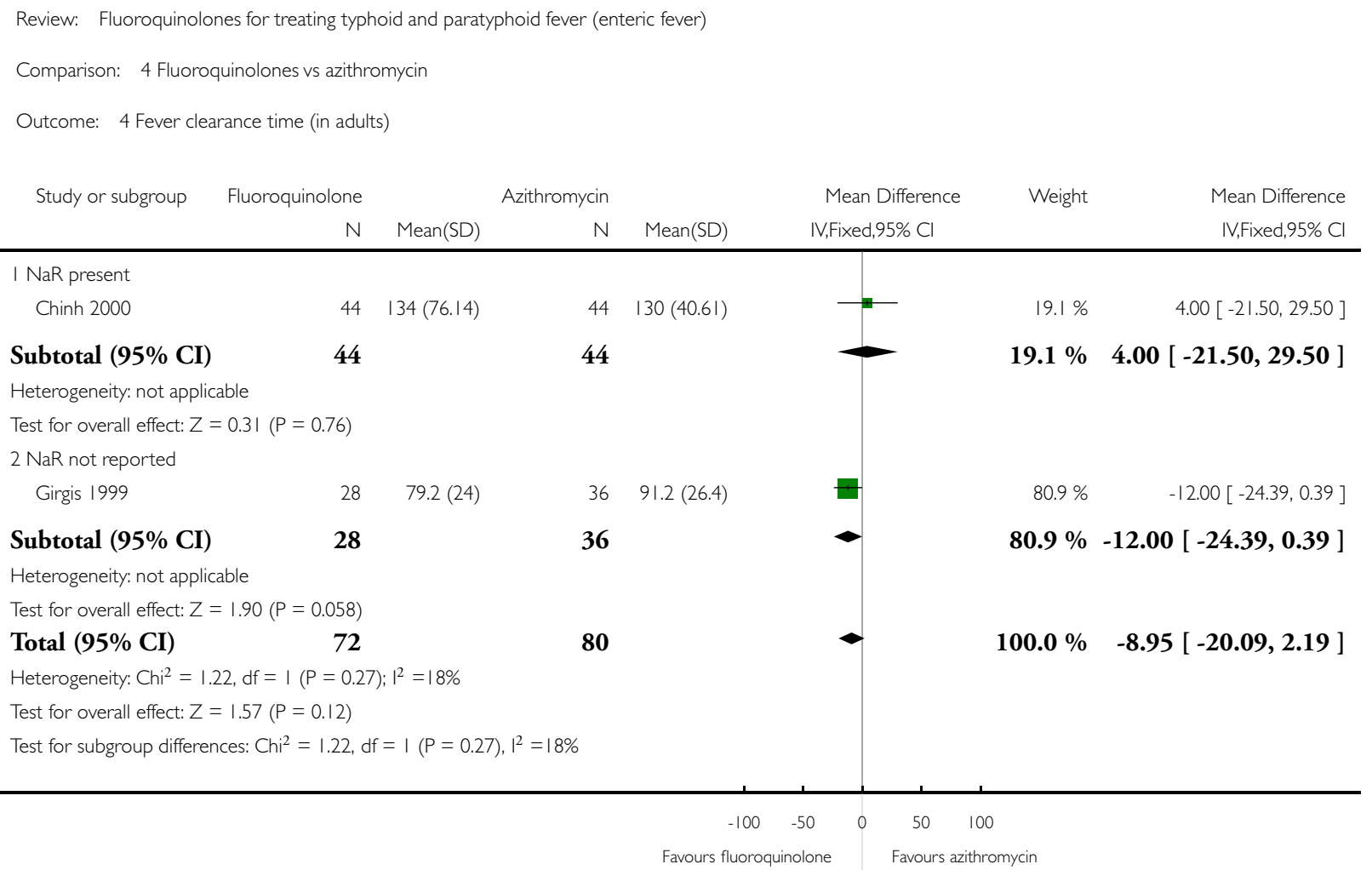


Analysis 4.5. Comparison 4 Fluoroquinolones vs azithromycin, Outcome 5 Length of hospital stay (days) (in adults).

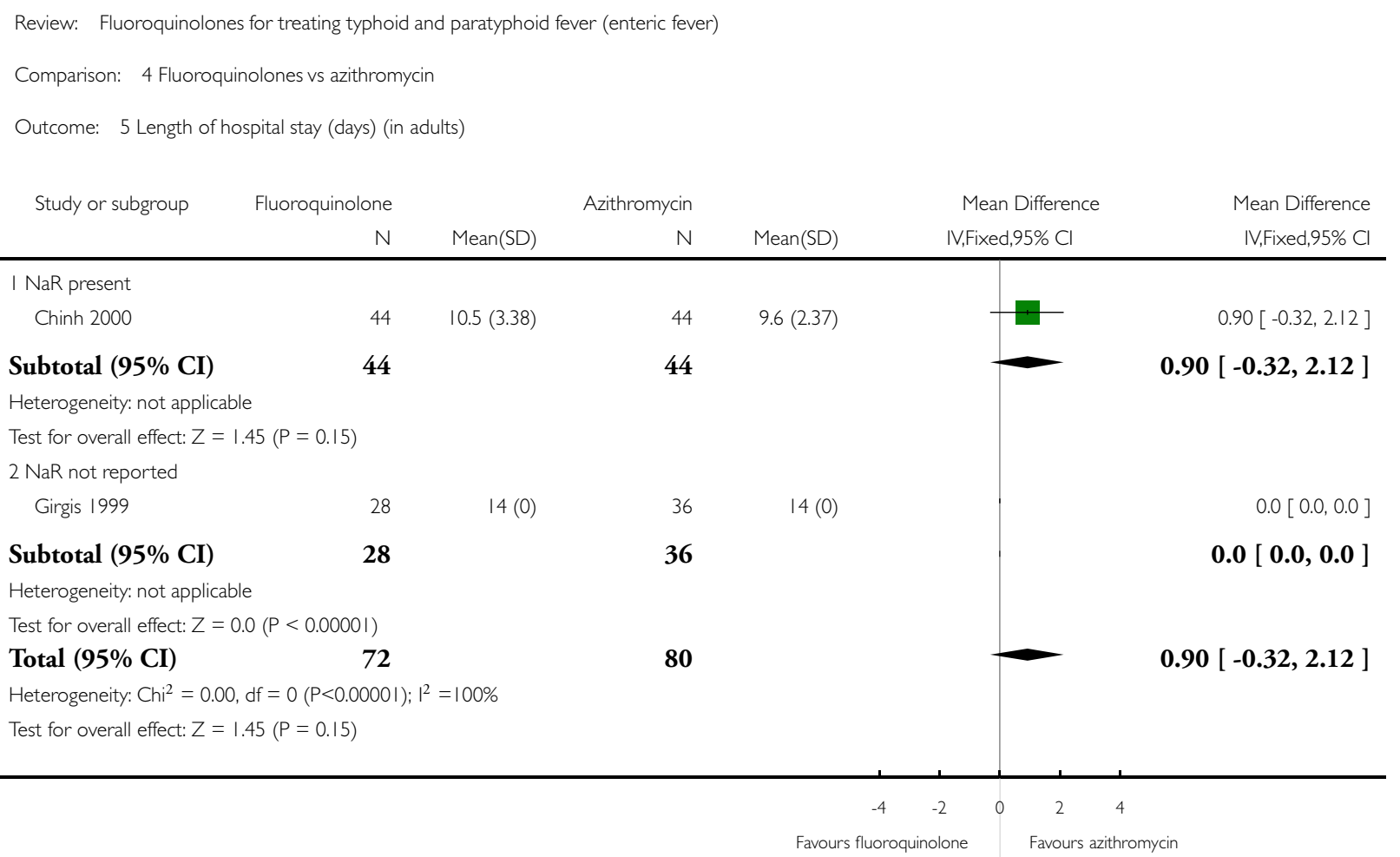


Analysis 4.6. Comparison 4 Fluoroquinolones vs azithromycin, Outcome 6 Convalescent faecal carriage (in adults).

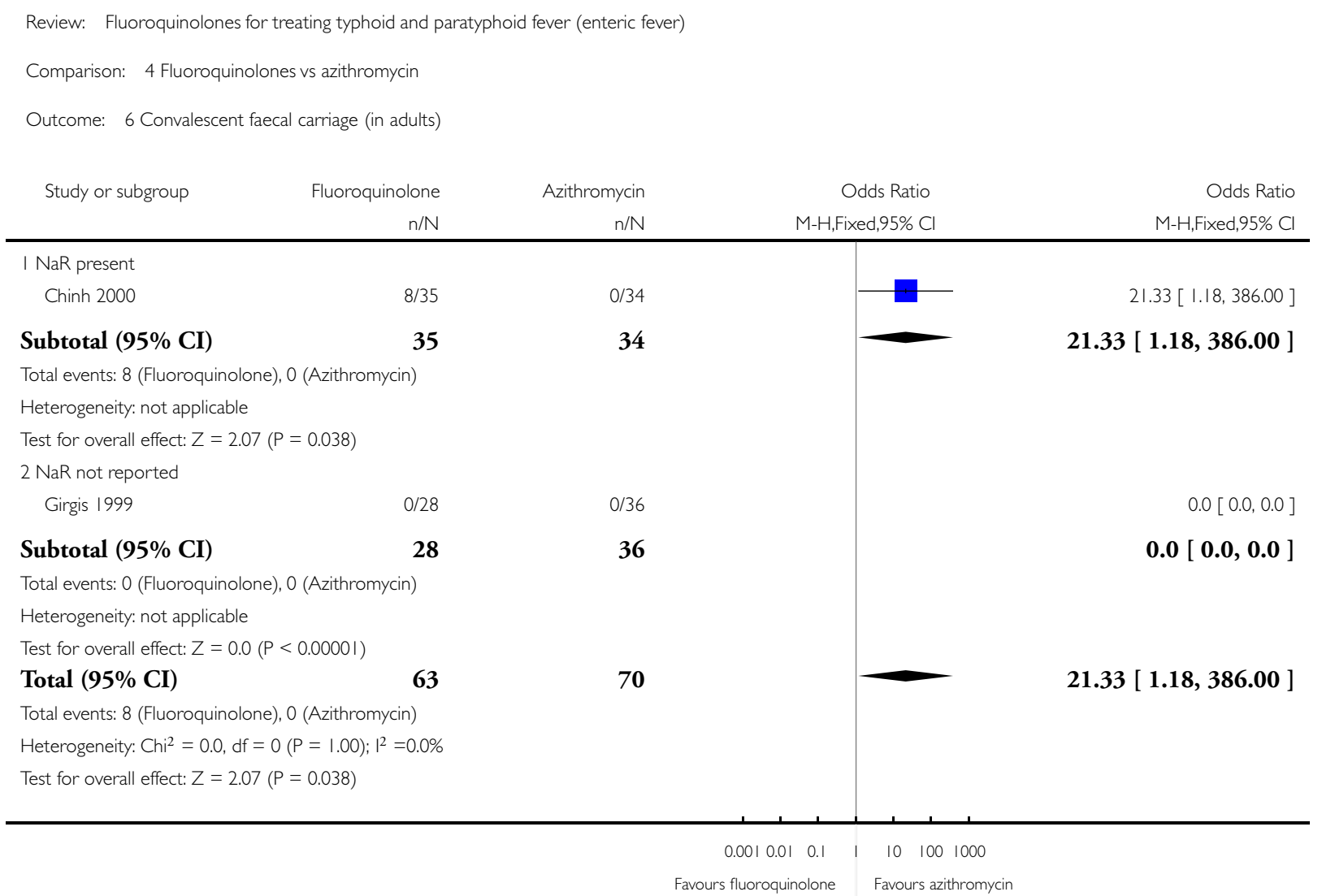




\section{Analysis 4.7. Comparison 4 Fluoroquinolones vs azithromycin, Outcome 7 Complications (in adults).}

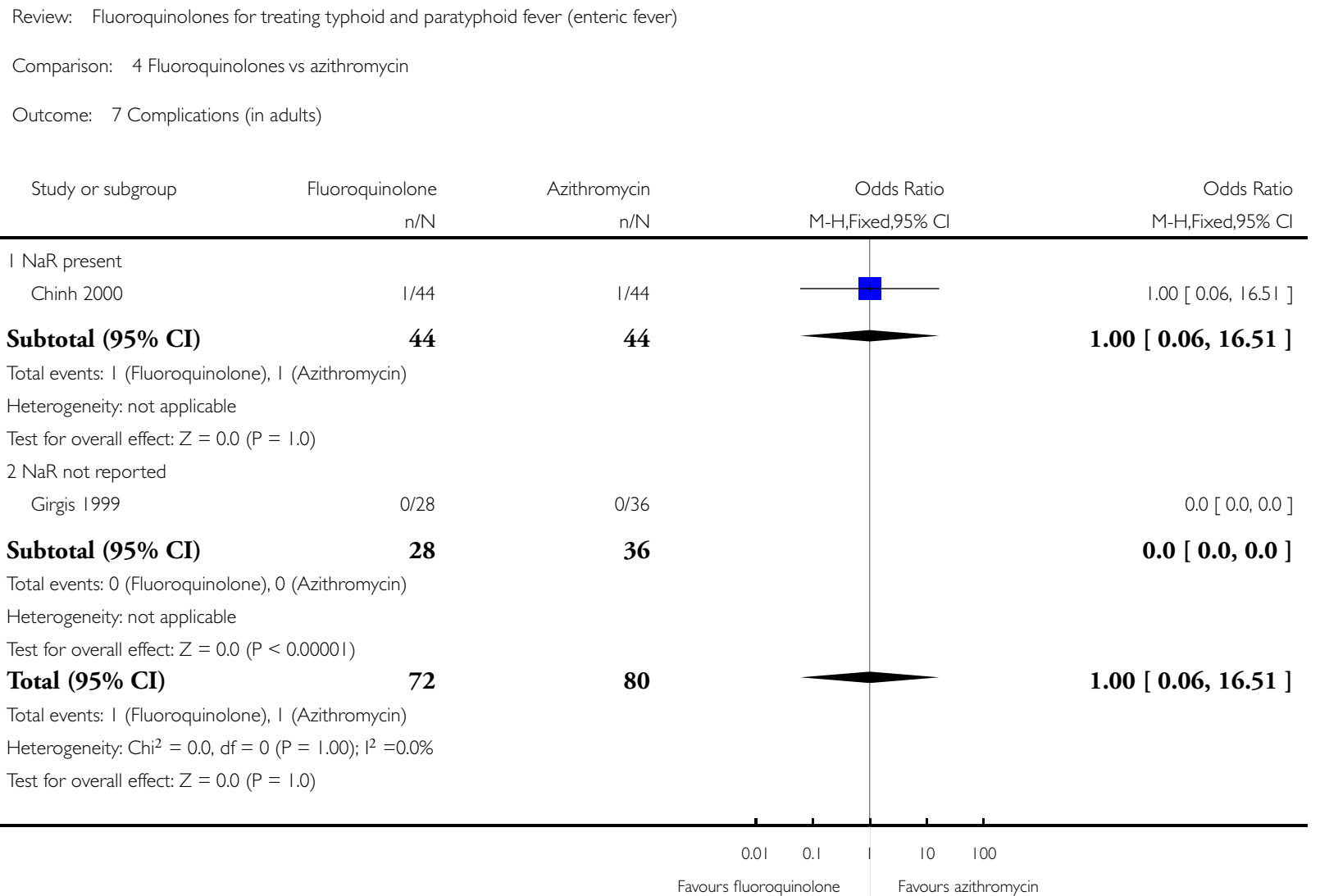

\section{Analysis 4.8. Comparison 4 Fluoroquinolones vs azithromycin, Outcome 8 Clinical failure (mostly children).}

Review: Fluoroquinolones for treating typhoid and paratyphoid fever (enteric fever)

Comparison: 4 Fluoroquinolones vs azithromycin

Outcome: 8 Clinical failure (mostly children)

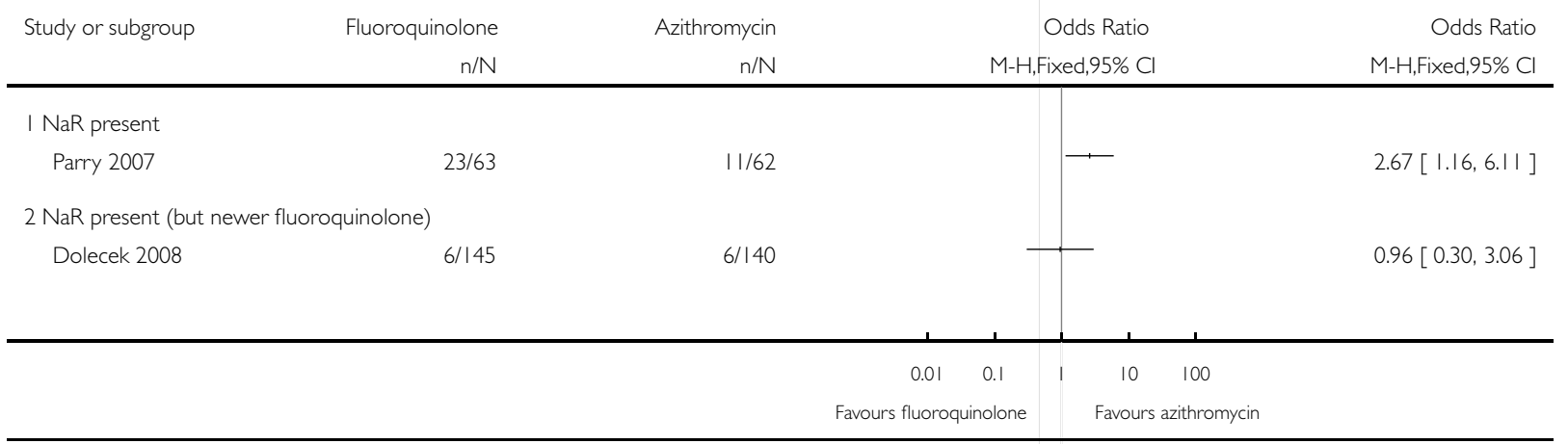




\section{Analysis 4.9. Comparison 4 Fluoroquinolones vs azithromycin, Outcome 9 Microbiological failure (mostly children).}

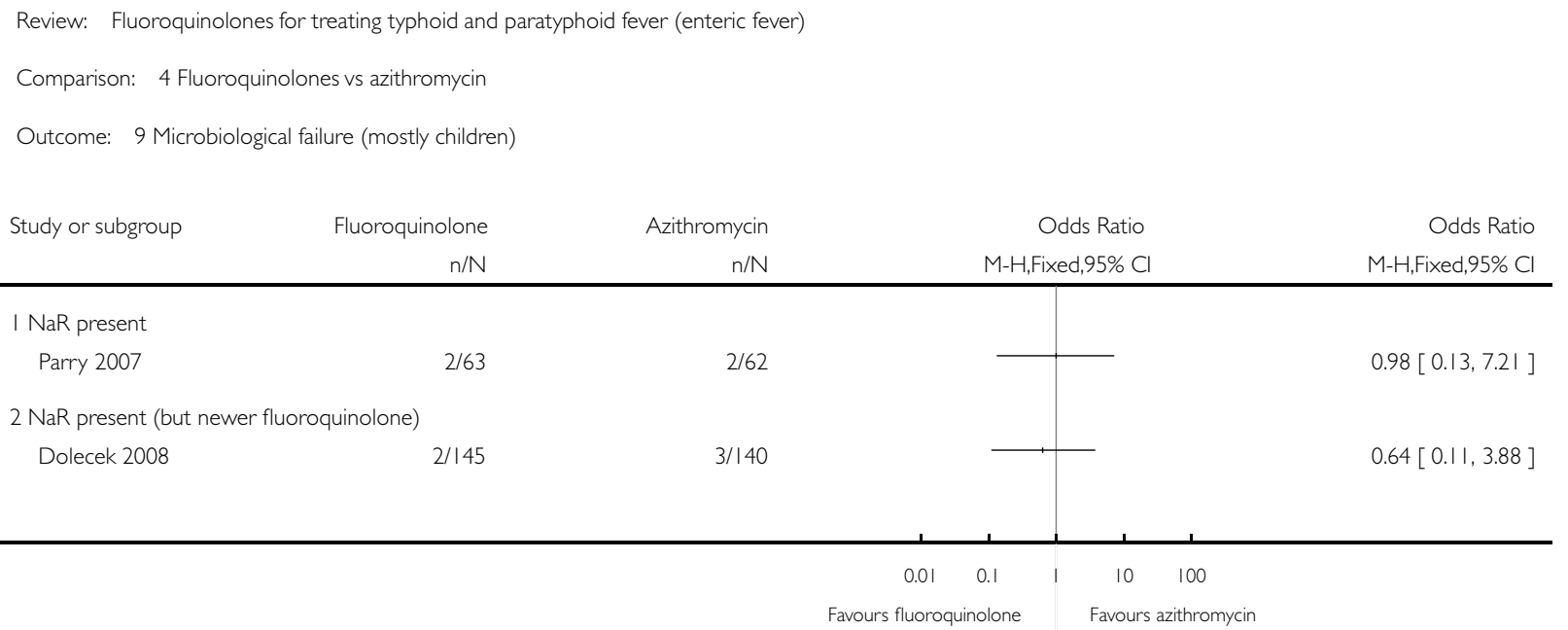

Analysis 4.10. Comparison 4 Fluoroquinolones vs azithromycin, Outcome 10 Relapse (mostly children).

Review: Fluoroquinolones for treating typhoid and paratyphoid fever (enteric fever)

Comparison: 4 Fluoroquinolones vs azithromycin

Outcome: 10 Relapse (mostly children)

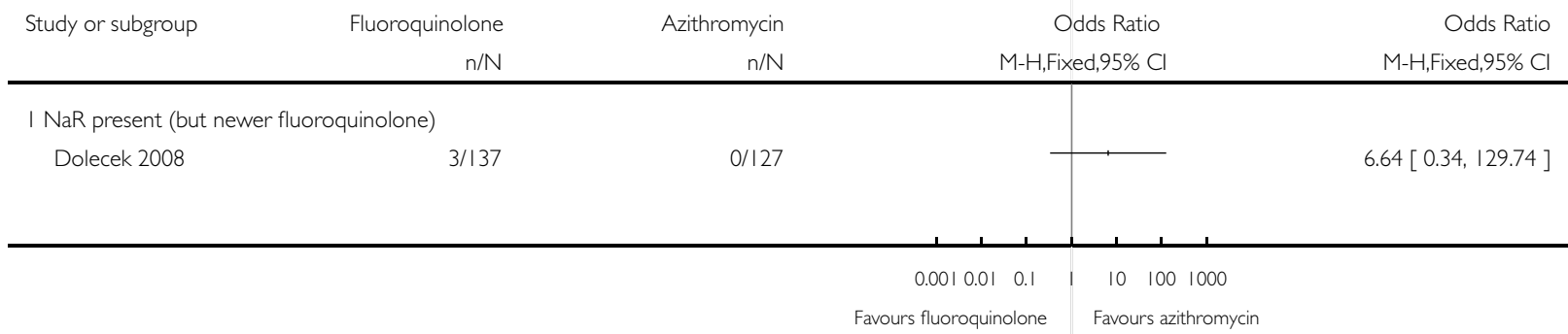




\section{Analysis 4. II. Comparison 4 Fluoroquinolones vs azithromycin, Outcome I I Fever clearance time (mostly children).}

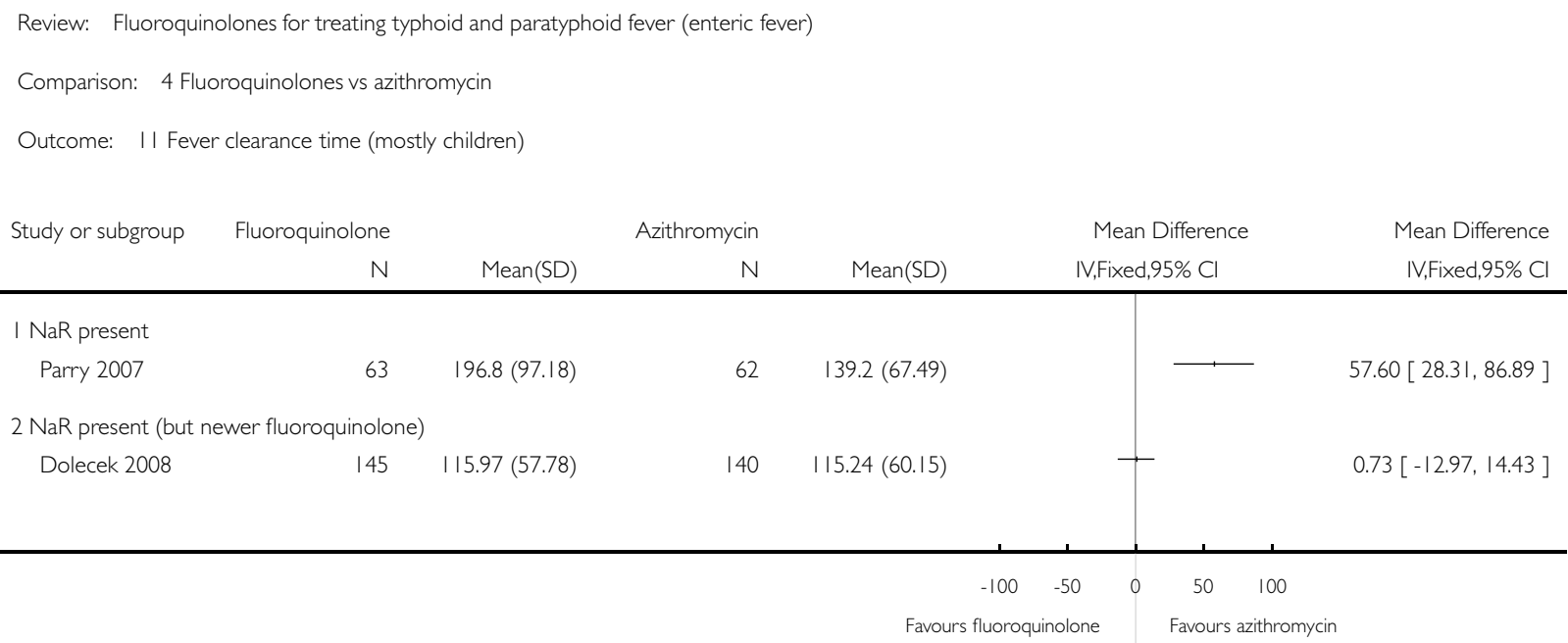

\section{Analysis 4.12. Comparison 4 Fluoroquinolones vs azithromycin, Outcome 12 Length of hospital stay} (mostly children).

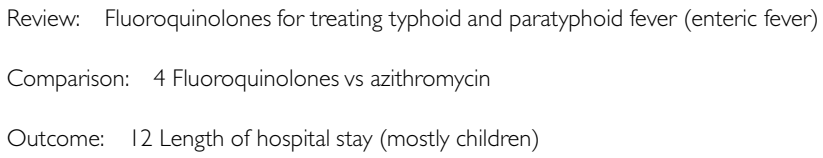

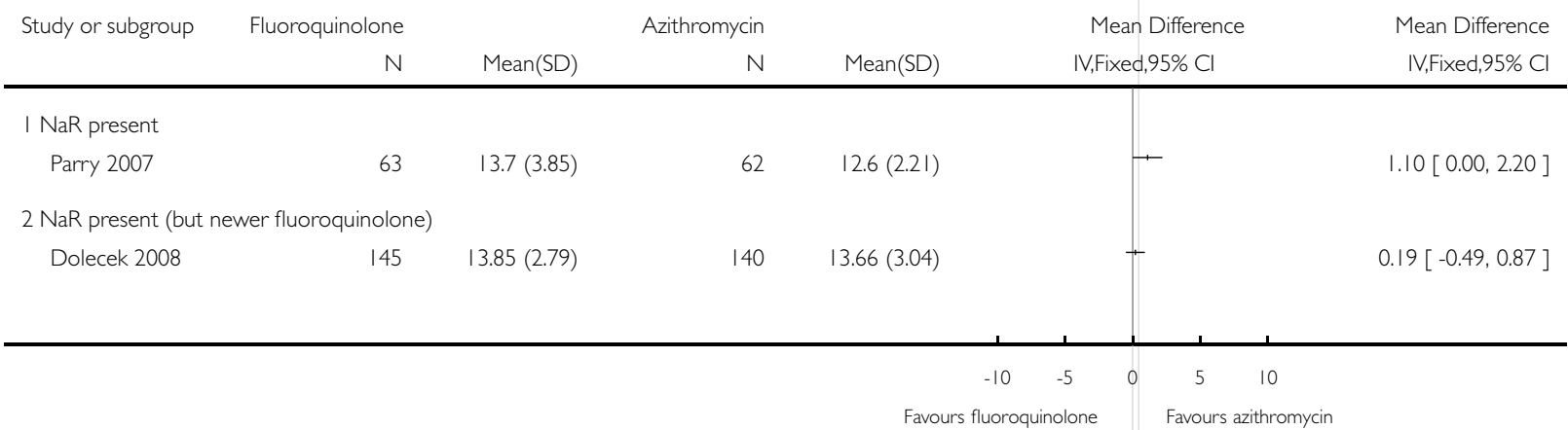




\section{Analysis 4.13. Comparison 4 Fluoroquinolones vs azithromycin, Outcome 13 Convalescent faecal carriage} (mostly children).

\begin{tabular}{|c|c|c|c|c|}
\hline \multicolumn{5}{|c|}{ Comparison: 4 Fluoroquinolones vs azithromycin } \\
\hline \multicolumn{5}{|c|}{ Outcome: 13 Convalescent faecal carriage (mostly children) } \\
\hline \multirow[t]{2}{*}{ Study or subgroup } & Fluoroquinolone & Azithromycin & Odds Ratio & Odds Ratio \\
\hline & $n / N$ & $n / N$ & M-H,Fixed,95\% Cl & M-H,Fixed,95\% Cl \\
\hline \multicolumn{5}{|l|}{ I NaR present } \\
\hline Parry 2007 & $12 / 62$ & 1/62 & $\longrightarrow$ & $14.64[1.84,116.48]$ \\
\hline \multicolumn{5}{|c|}{$2 \mathrm{NaR}$ present (but newer fluoroquinolone) } \\
\hline Dolecek 2008 & $1 / 137$ & $0 / 131$ & 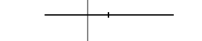 & $2.89[0.12,71.58]$ \\
\hline
\end{tabular}

\section{Analysis 4.I4. Comparison 4 Fluoroquinolones vs azithromycin, Outcome 14 Complications (mostly} children).

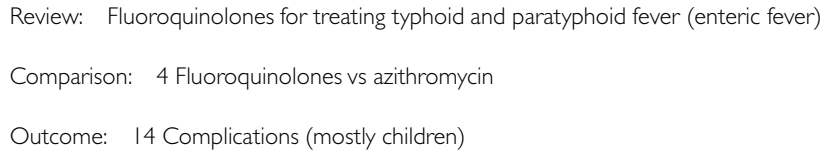

n/N $n / N \quad M-H, F i x e d, 95 \%$ Cl

$2 \mathrm{NaR}$ present (but newer fluoroquinolone) 


\section{Analysis 4.15. Comparison 4 Fluoroquinolones vs azithromycin, Outcome I5 Adverse events (not serious)} (mostly children).

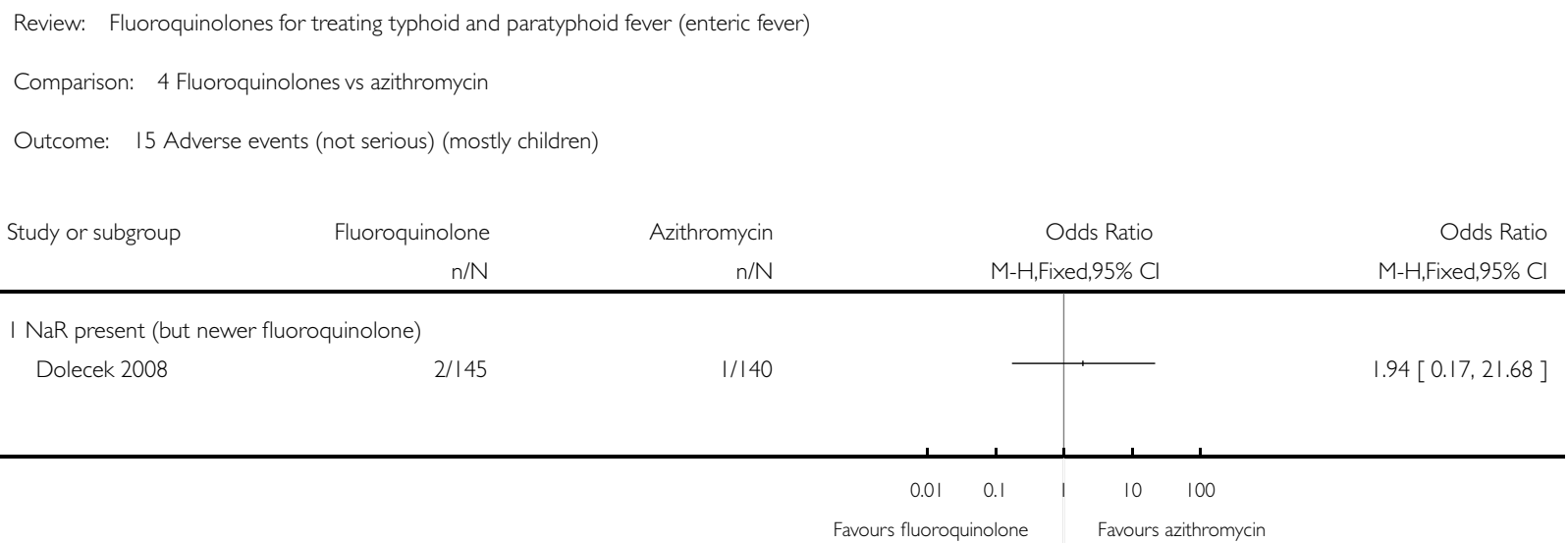

\section{Analysis 5.I. Comparison 5 Fluoroquinolones vs cefixime, Outcome I Clinical failure (adults or mostly} adults).

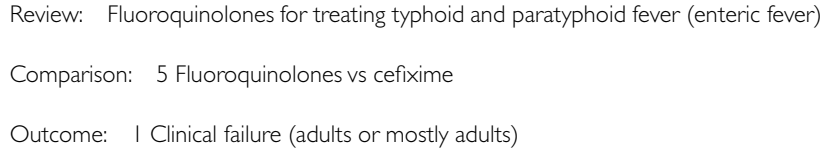

Yu 1998

Subtotal $(95 \% \mathrm{CI})$

Total events: 0 (Fluoroquinolone), 3 (Cefixime)

Heterogeneity: not applicable

Test for overall effect: $Z=1.32(P=0.19)$

$2 \mathrm{NaR}$ present (but newer fluoroquinolone)

Pandit 2007 $1 / 88$

Subtotal $(95 \% \mathrm{CI})$ 88

Total events: I (Fluoroquinolone), 19 (Cefixime)

Heterogeneity: not applicable

Test for overall effect: $Z=3.34$ ( $P=0.00083)$

Total (95\% CI)

128

Total events: I (Fluoroquinolone), 22 (Cefixime)

Heterogeneity: $\mathrm{Ch}^{2}{ }^{2}=0.63, \mathrm{df}=\mathrm{I}(\mathrm{P}=0.43) ; \mathrm{I}^{2}=0.0 \%$

Test for overall effect: $Z=3.66(P=0.00025)$

$\mathrm{n} / \mathrm{N}$

$0 / 40 \quad 3 / 40$

40

40

40

$\longrightarrow$

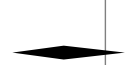

$14.2 \%$

$0.13[0.01,2.65]$

$14.2 \%$

$0.13[0.01,2.65]$

(2)




\section{Analysis 5.2. Comparison 5 Fluoroquinolones vs cefixime, Outcome 2 Microbiological failure (adults or}

mostly adults).

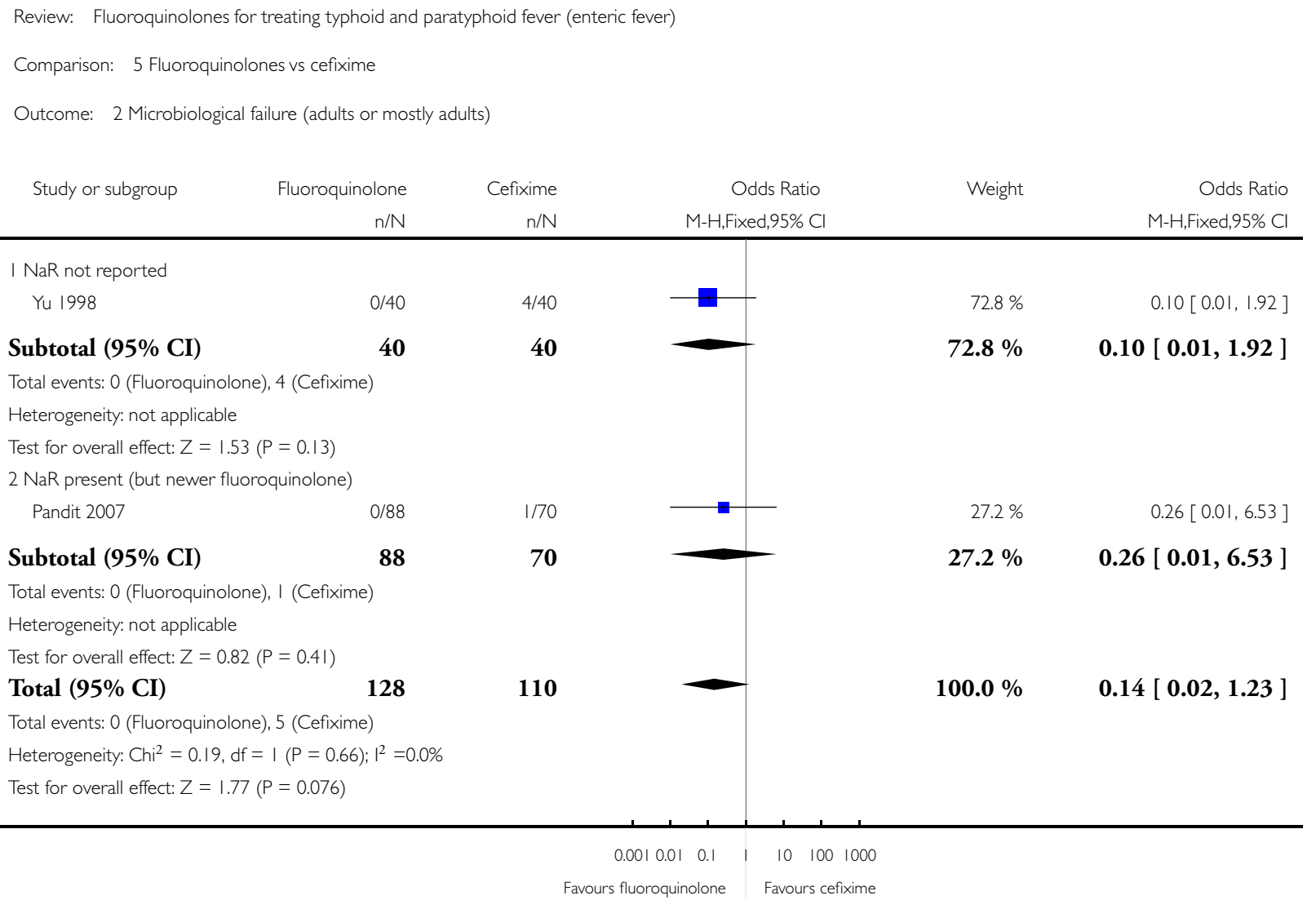


Analysis 5.3. Comparison 5 Fluoroquinolones vs cefixime, Outcome 3 Relapse (adults or mostly adults).

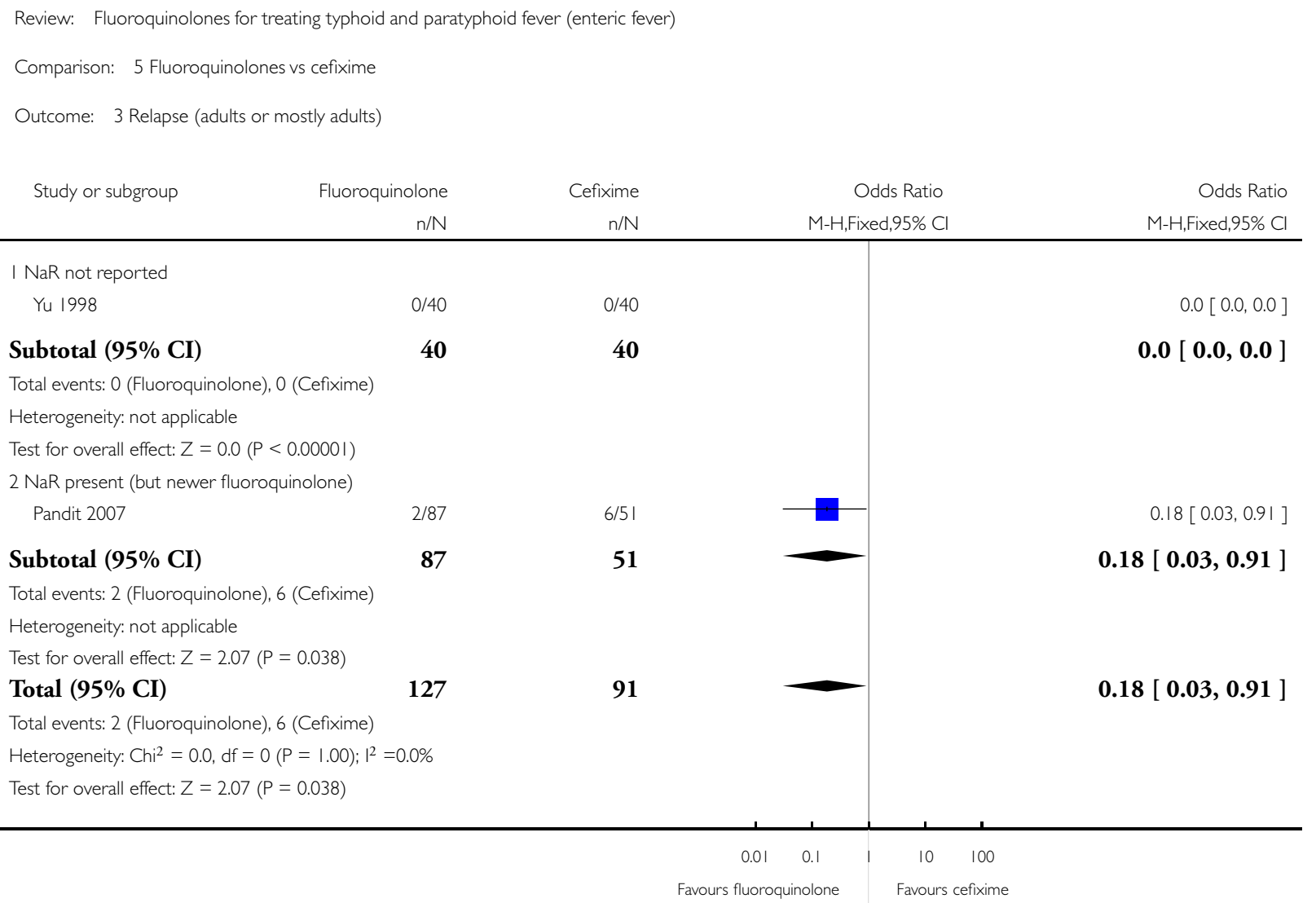


Analysis 5.4. Comparison 5 Fluoroquinolones vs cefixime, Outcome 4 Fever clearance time (adults or mostly adults).

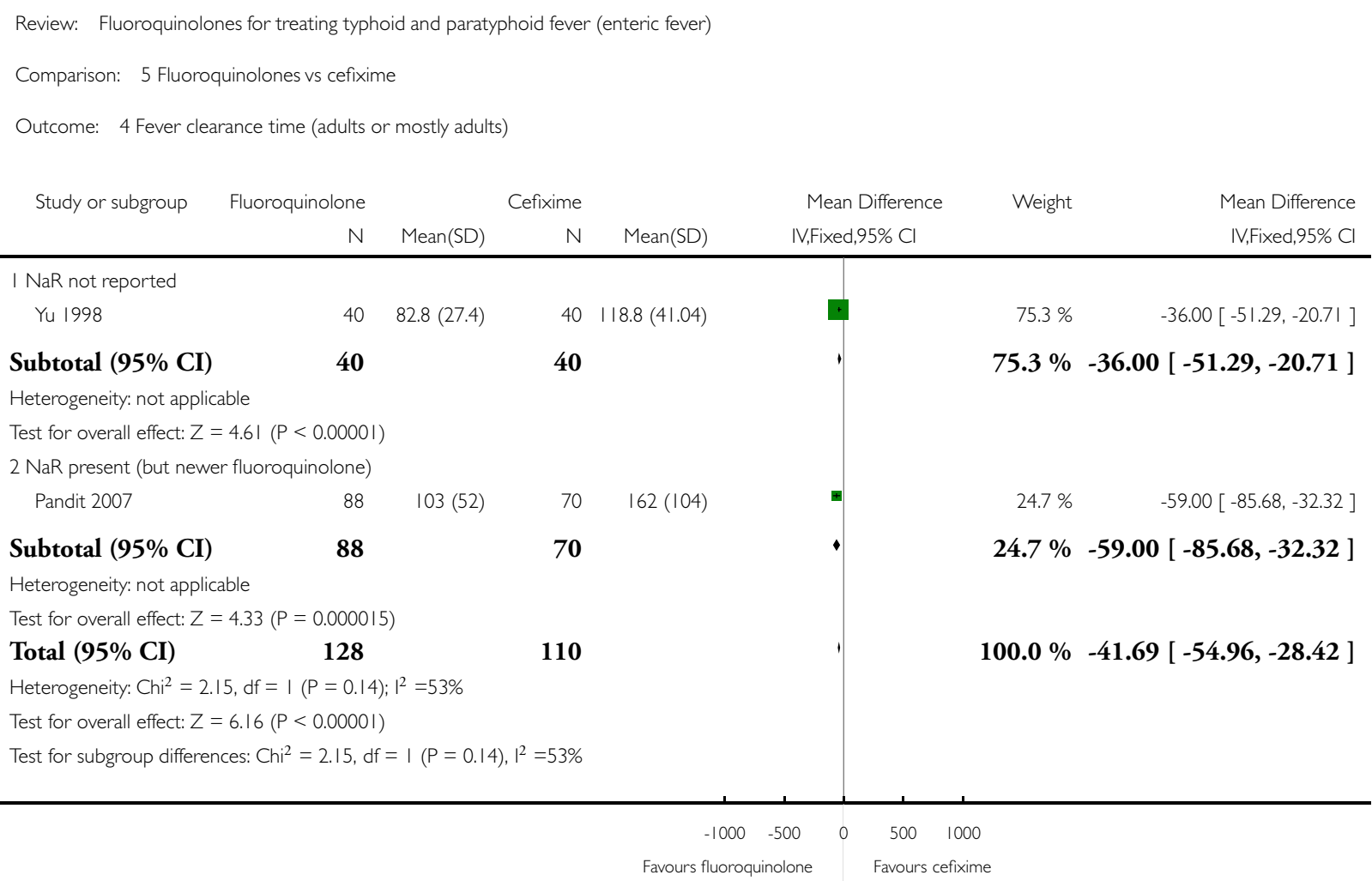


Analysis 5.5. Comparison 5 Fluoroquinolones vs cefixime, Outcome 5 Convalescent faecal carriage (adults or mostly adults).

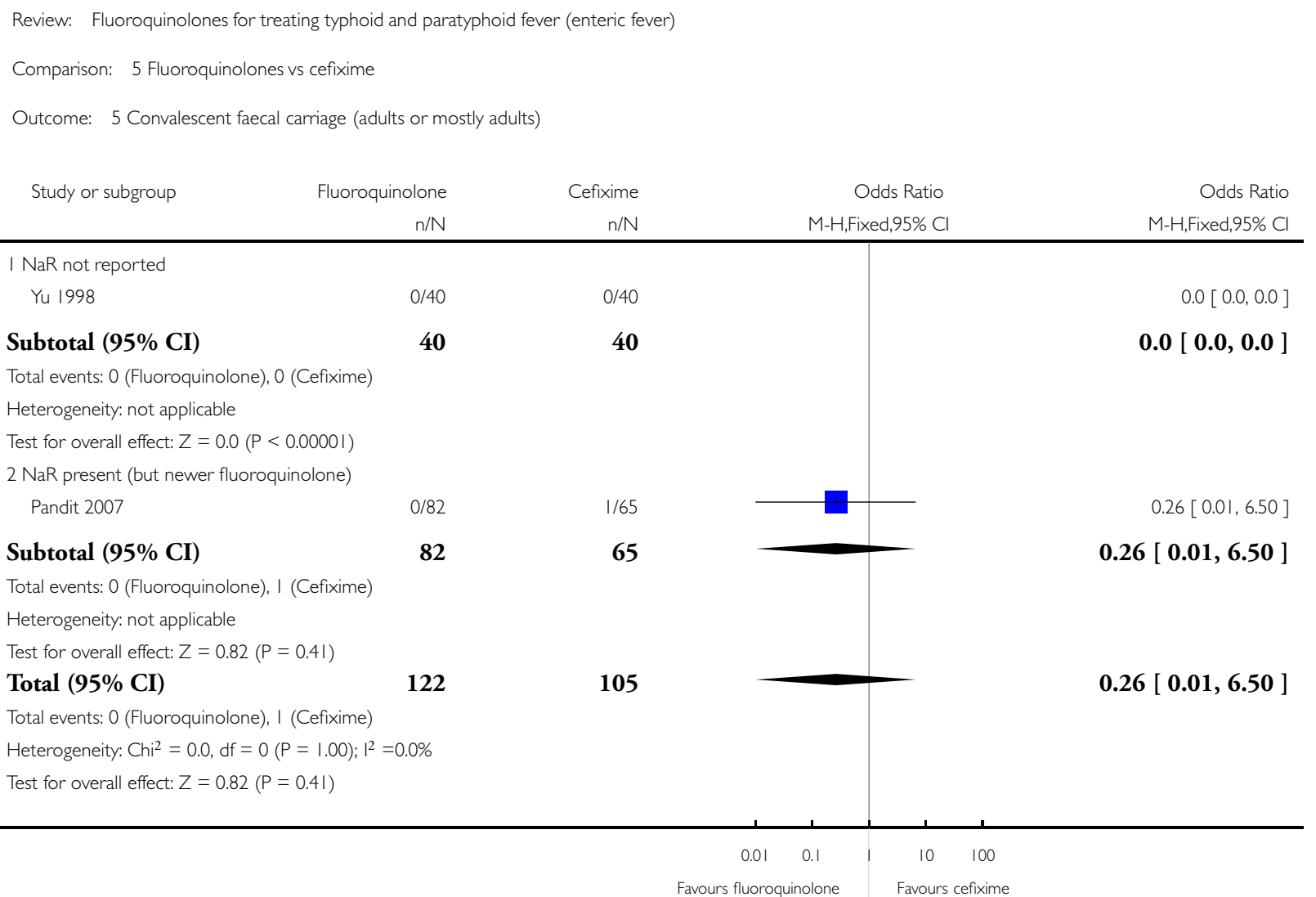

\section{Analysis 5.6. Comparison 5 Fluoroquinolones vs cefixime, Outcome 6 Complications (adults or mostly adults).}

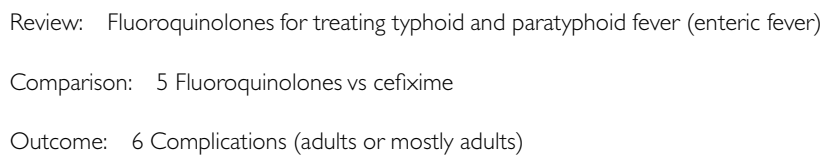

I NaR present (but newer fluoroquinolone) Pandit 2007 
Analysis 5.7. Comparison 5 Fluoroquinolones vs cefixime, Outcome 7 Adverse events (not serious) (adults or mostly adults).

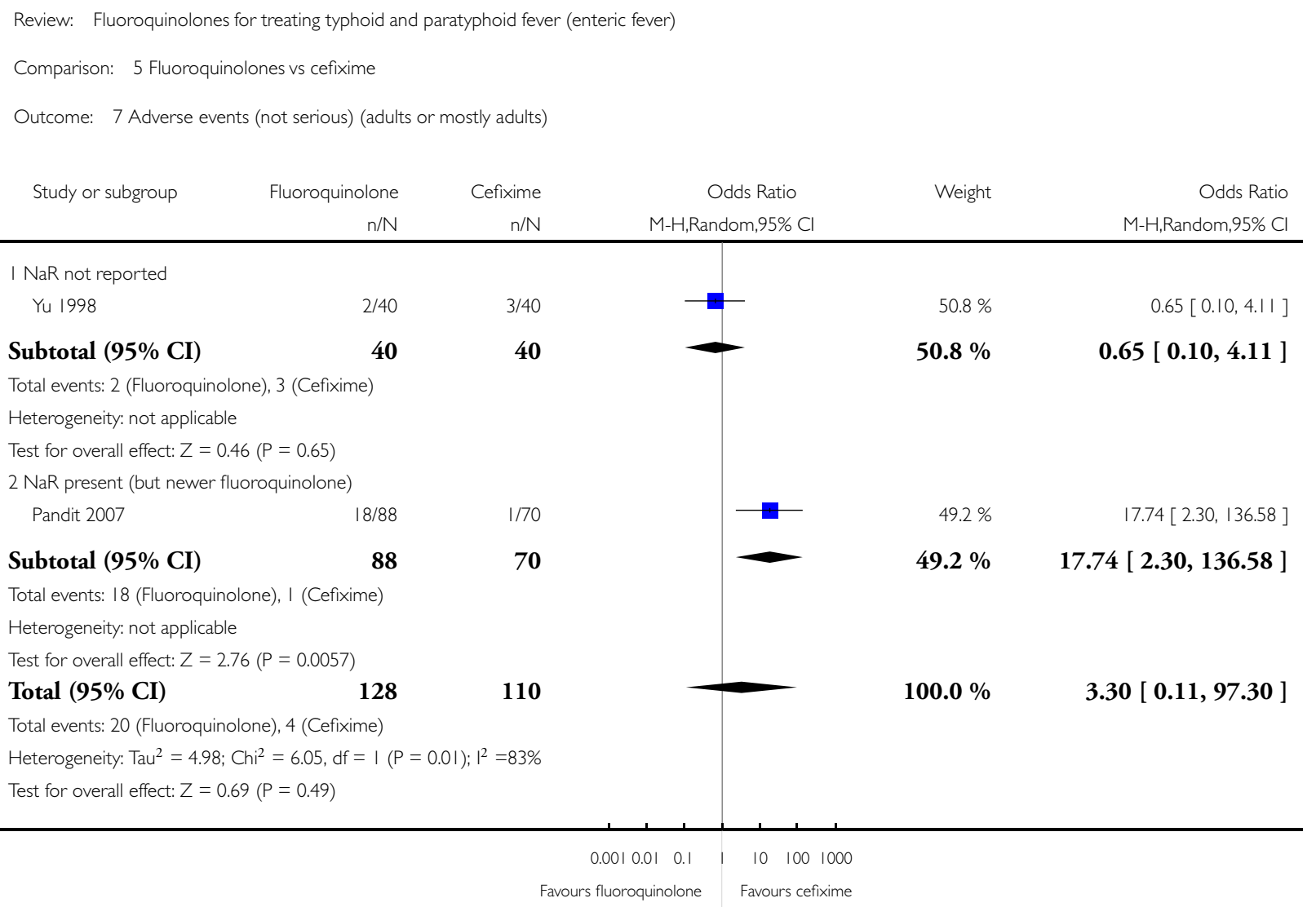


Analysis 5.8. Comparison 5 Fluoroquinolones vs cefixime, Outcome 8 Clinical failure (children only).

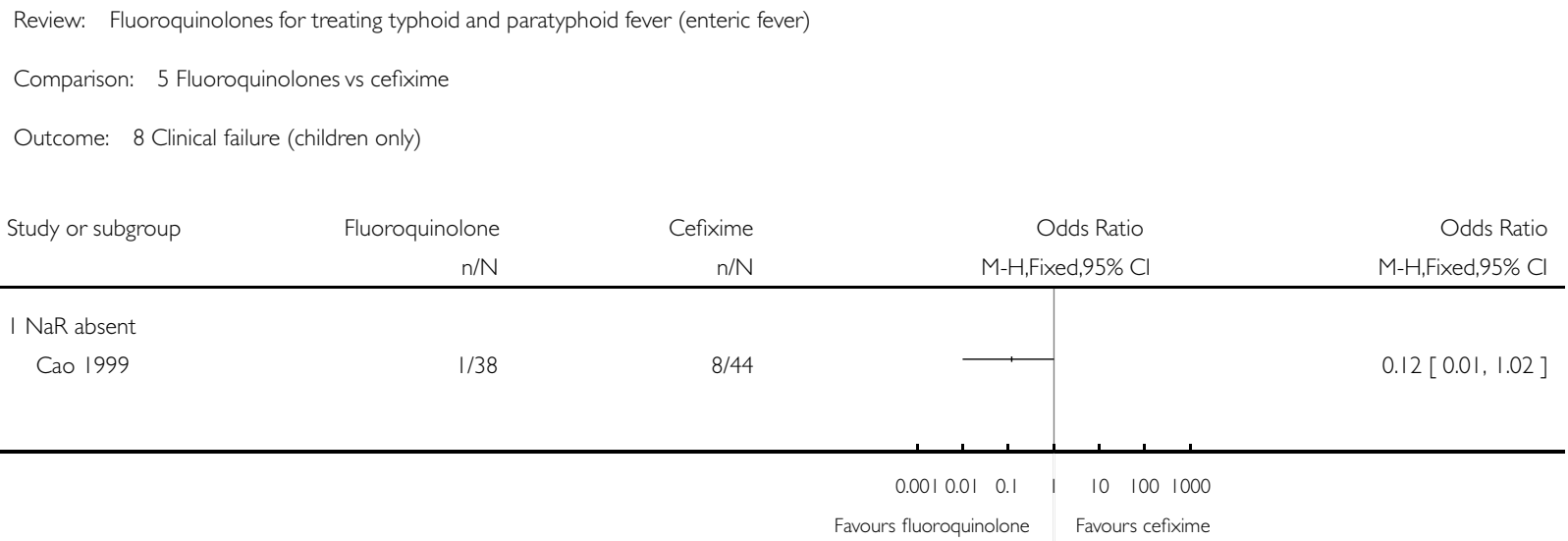

Analysis 5.9. Comparison 5 Fluoroquinolones vs cefixime, Outcome 9 Microbiological failure (children only).

Review: Fluoroquinolones for treating typhoid and paratyphoid fever (enteric fever)

Comparison: 5 Fluoroquinolones vs cefixime

Outcome: 9 Microbiological failure (children only)

\begin{tabular}{|c|c|c|c|c|}
\hline \multirow{2}{*}{ Study or subgroup } & Fluoroquinolone & Cefixime & \multirow{2}{*}{$\begin{array}{r}\text { Odds Ratio } \\
\text { M-H,Fixed,95\% Cl }\end{array}$} & Odds Ratio \\
\hline & $n / N$ & $\mathrm{n} / \mathrm{N}$ & & M-H,Fixed,95\% Cl \\
\hline \multicolumn{5}{|l|}{ I NaR absent } \\
\hline Cao 1999 & $0 / 38$ & $2 / 44$ & - & $0.22[0.01,4.74]$ \\
\hline
\end{tabular}




\section{Analysis 5.10. Comparison 5 Fluoroquinolones vs cefixime, Outcome 10 Relapse (children only).}

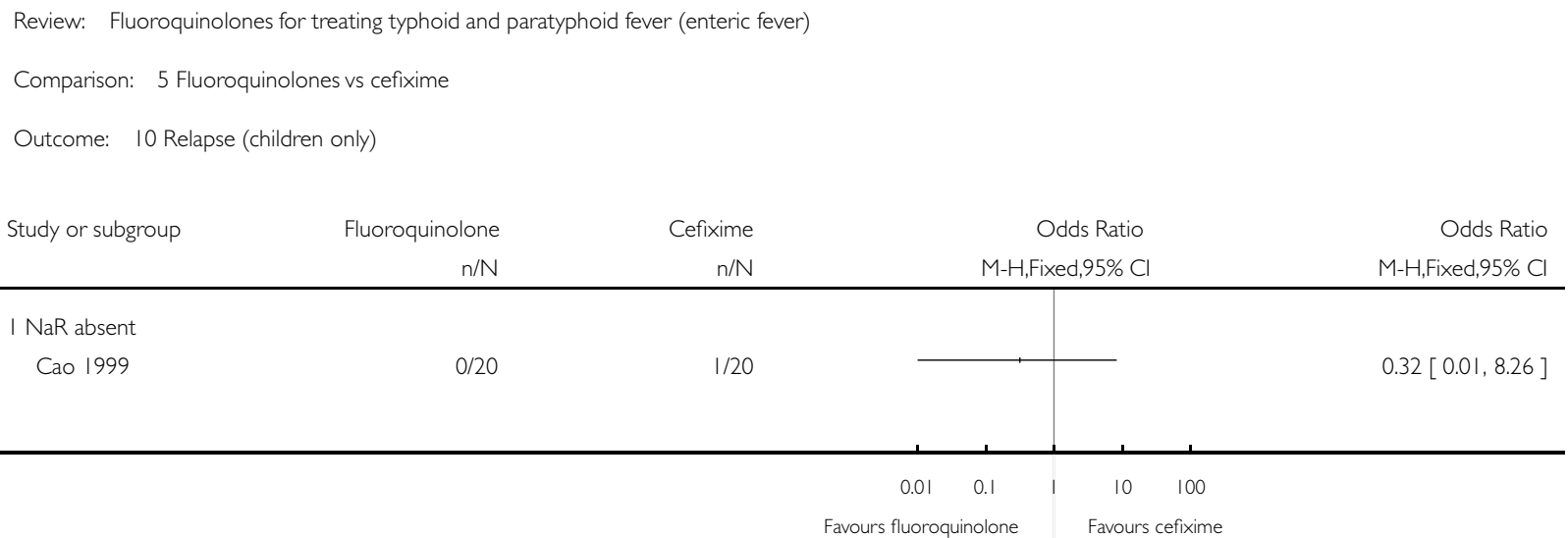

Analysis 5.I I. Comparison 5 Fluoroquinolones vs cefixime, Outcome II Fever clearance time (children only).

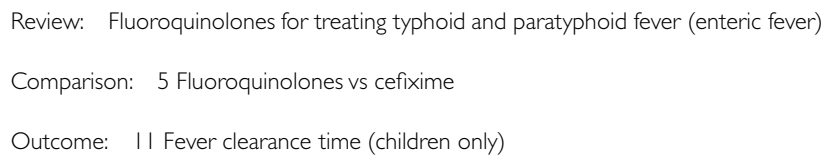

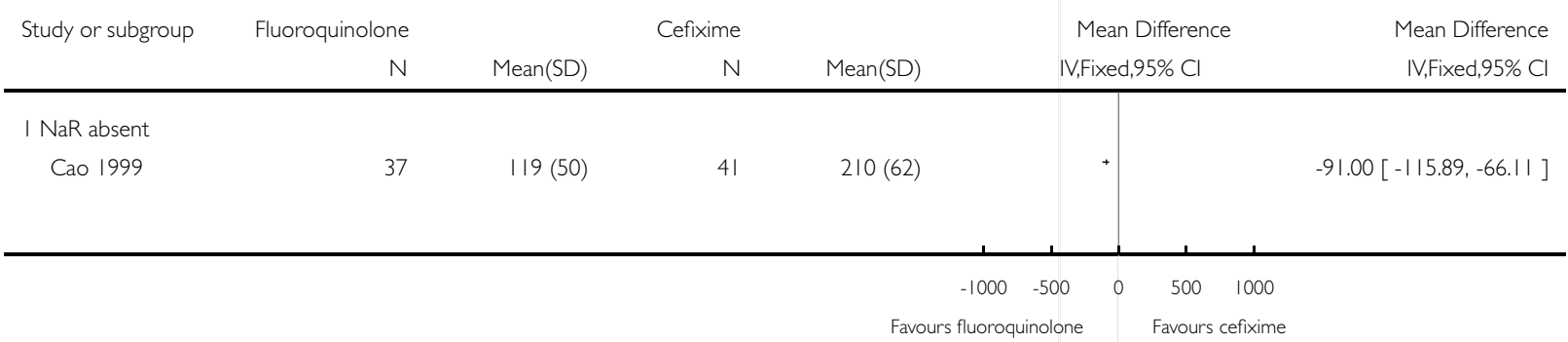




\section{Analysis 5.12. Comparison 5 Fluoroquinolones vs cefixime, Outcome 12 Length of hospital stay (children} only).

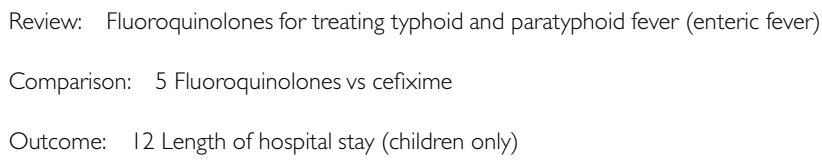

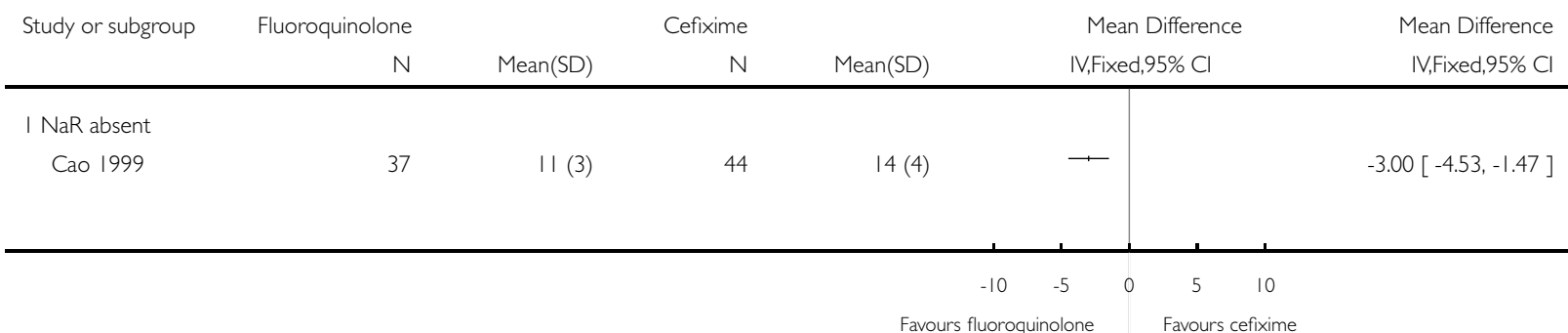

Analysis 5.13. Comparison 5 Fluoroquinolones vs cefixime, Outcome I 3 Complications (children only).

Review: Fluoroquinolones for treating typhoid and paratyphoid fever (enteric fever)

Comparison: 5 Fluoroquinolones vs cefixime

Outcome: 13 Complications (children only)

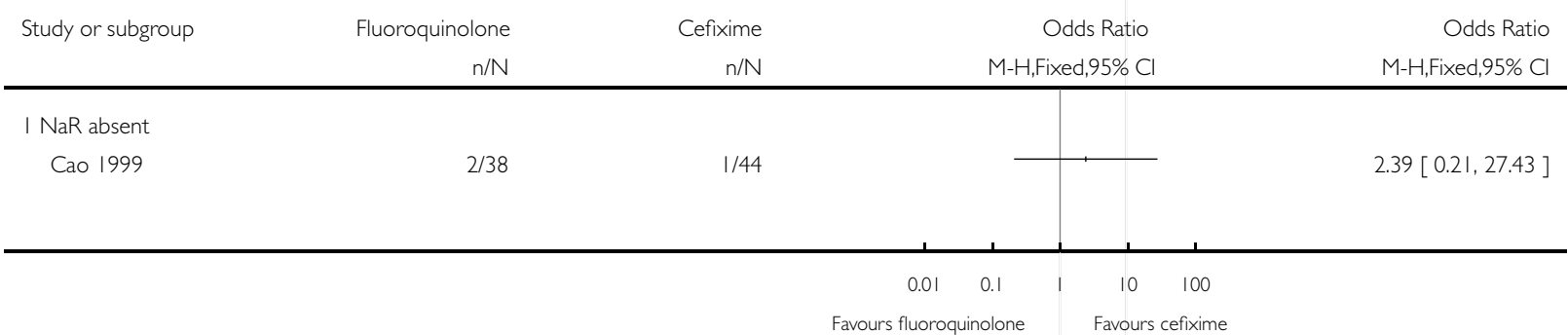




\section{Analysis 6.1. Comparison 6 Fluoroquinolones vs ceftriaxone, Outcome I Clinical failure.}

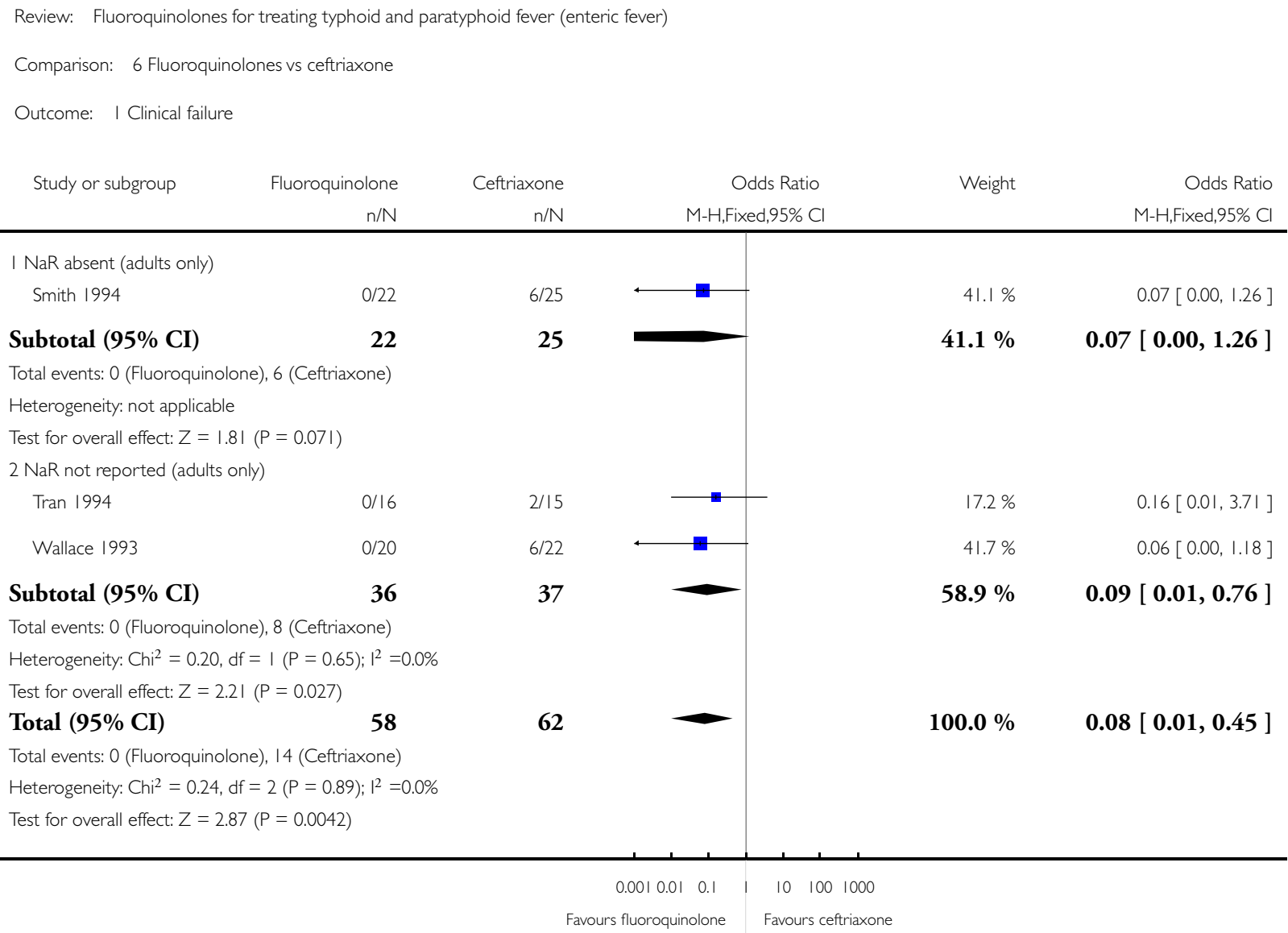




\section{Analysis 6.2. Comparison 6 Fluoroquinolones vs ceftriaxone, Outcome 2 Microbiological failure.}

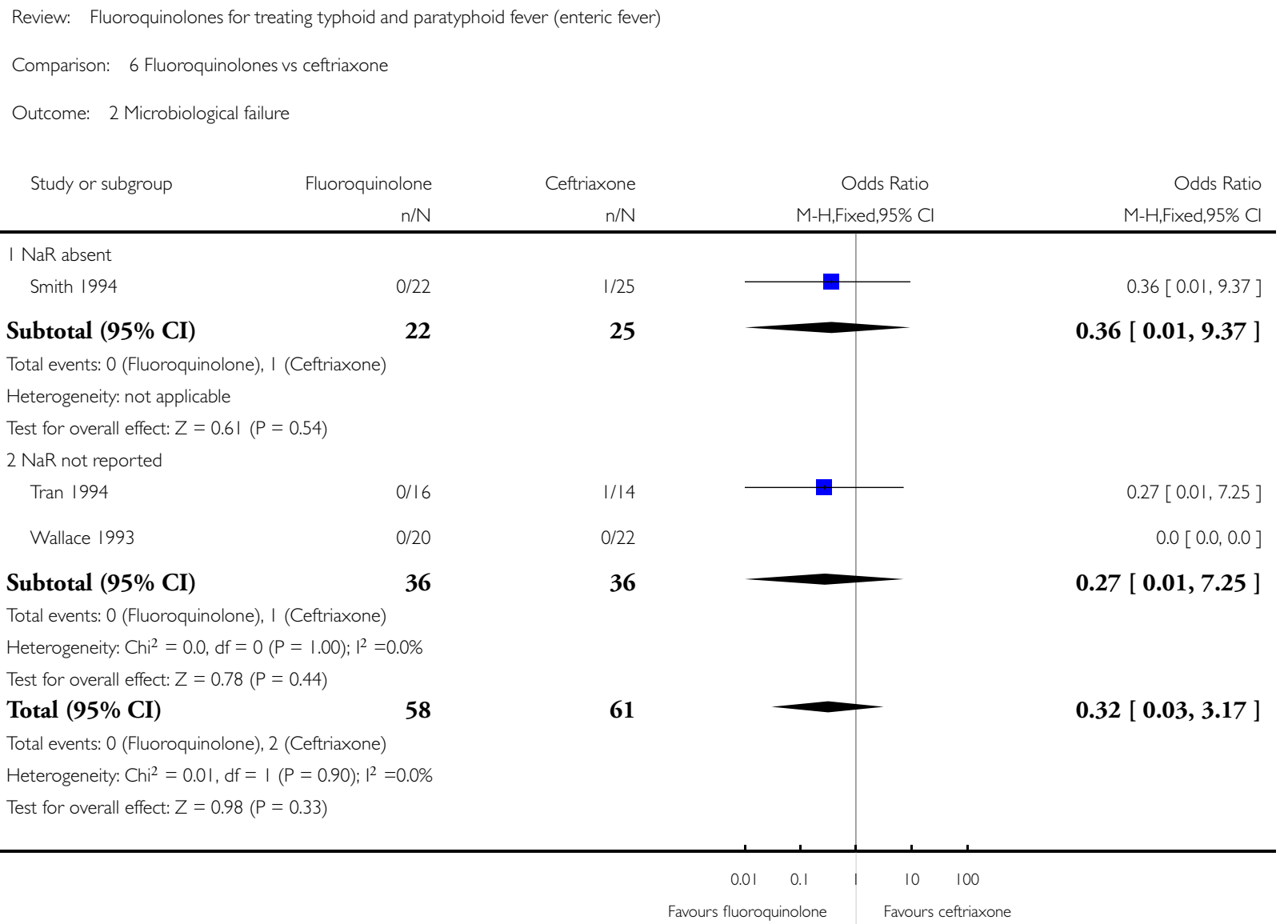




\section{Analysis 6.3. Comparison 6 Fluoroquinolones vs ceftriaxone, Outcome 3 Relapse.}

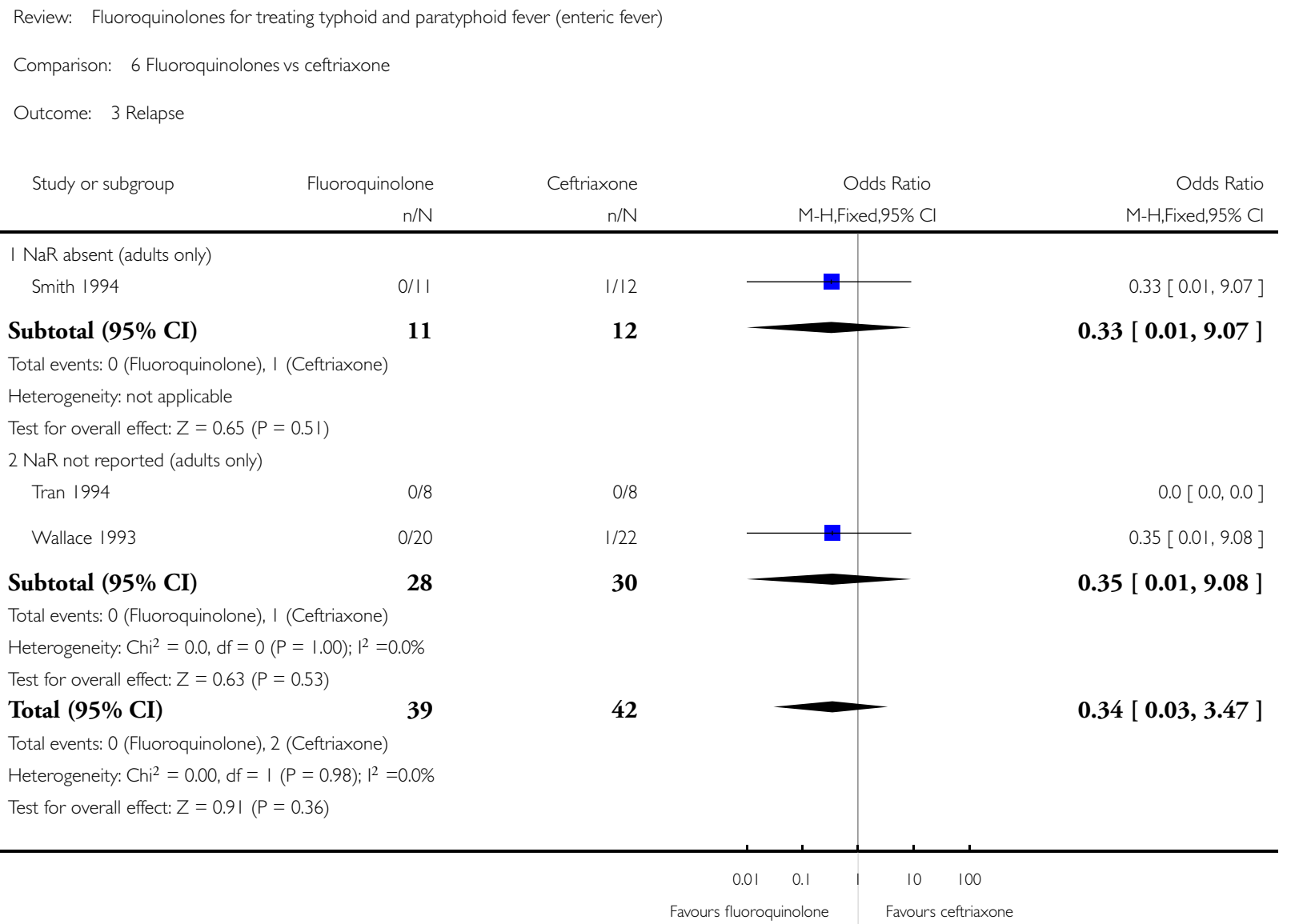




\section{Analysis 6.4. Comparison 6 Fluoroquinolones vs ceftriaxone, Outcome 4 Fever clearance time.}

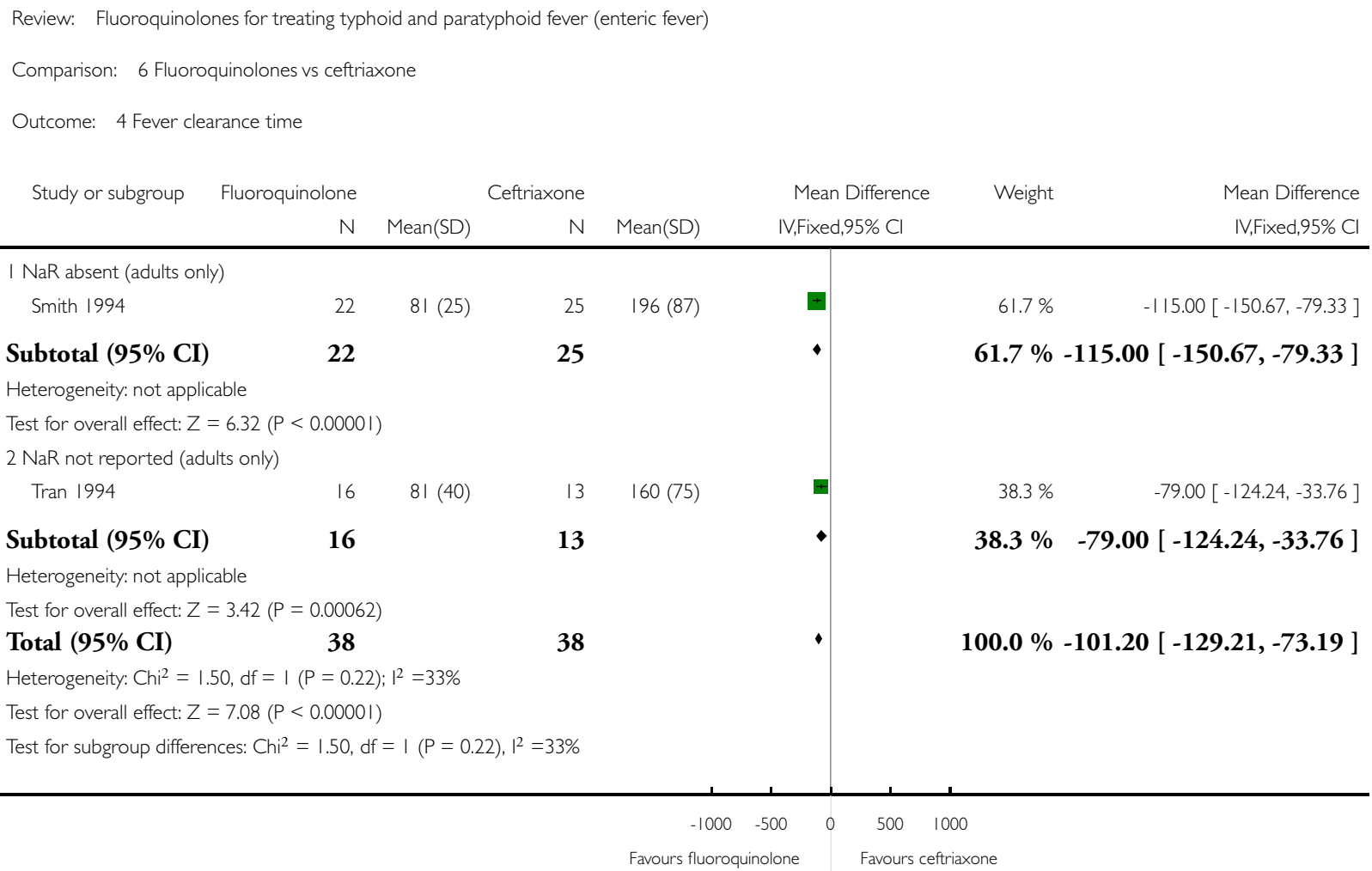




\section{Analysis 6.5. Comparison 6 Fluoroquinolones vs ceftriaxone, Outcome 5 Convalescent faecal carriage.}

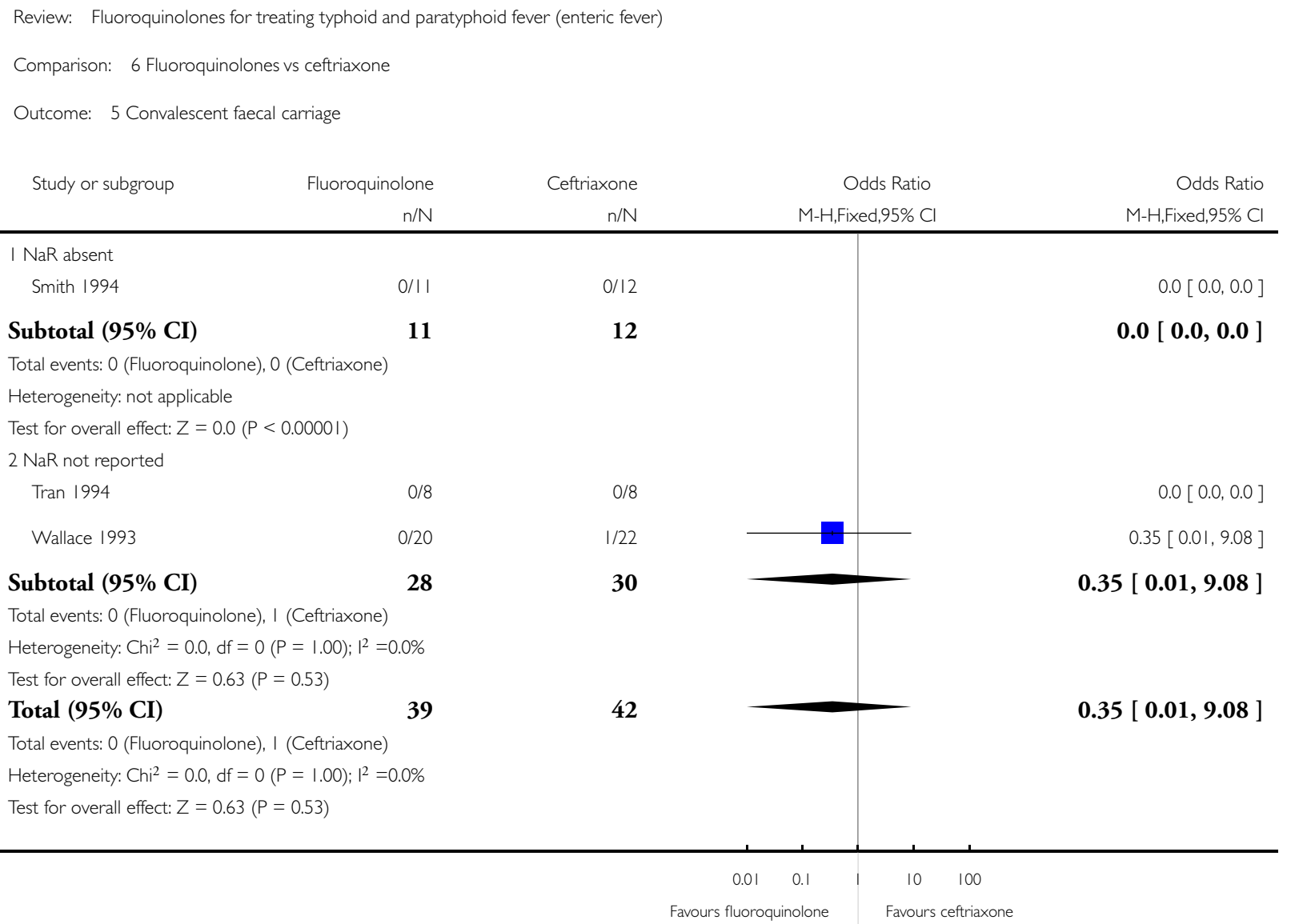




\section{Analysis 6.6. Comparison 6 Fluoroquinolones vs ceftriaxone, Outcome 6 Complications.}

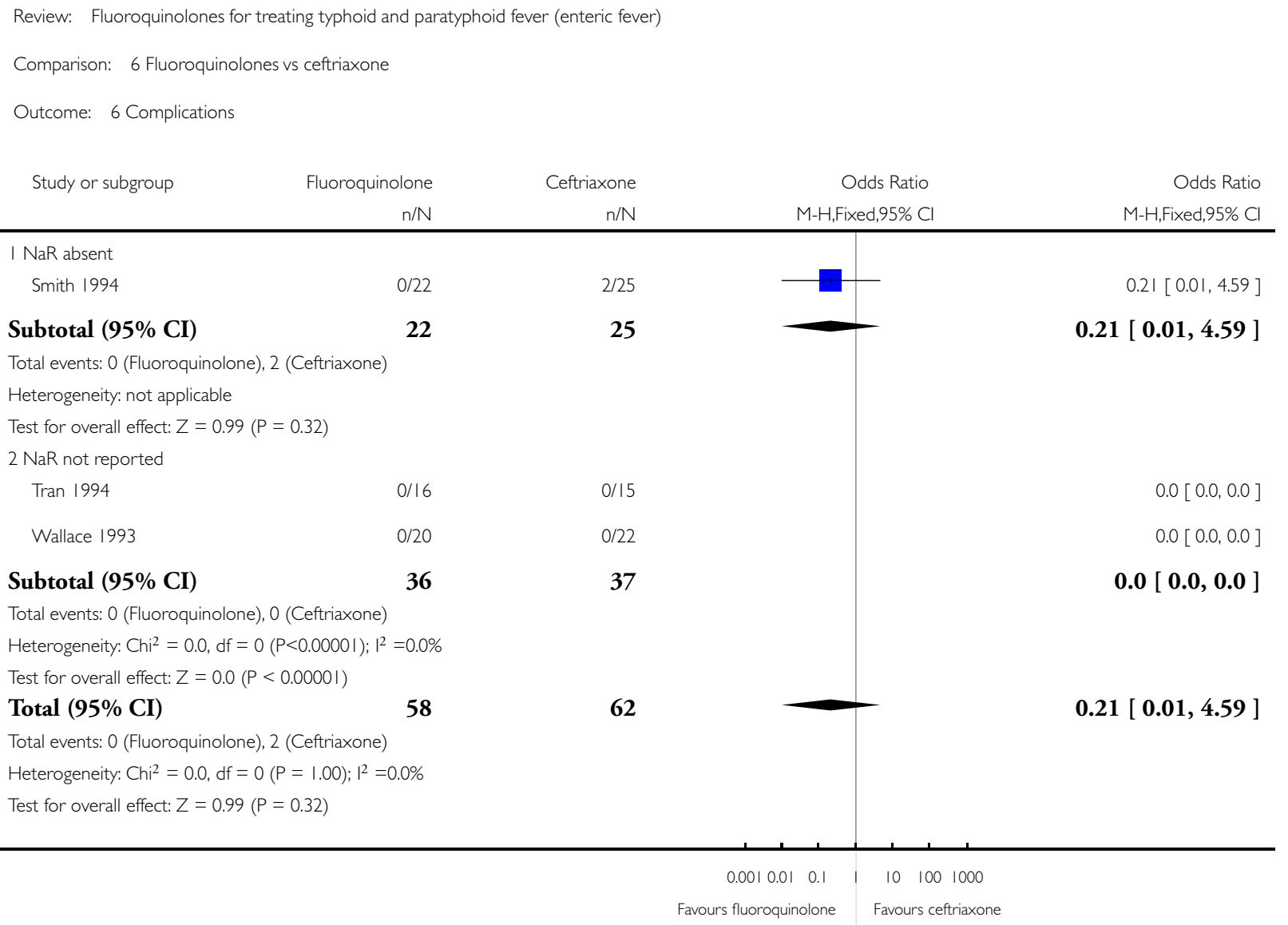




\section{Analysis 6.7. Comparison 6 Fluoroquinolones vs ceftriaxone, Outcome 7 Serious adverse events.}

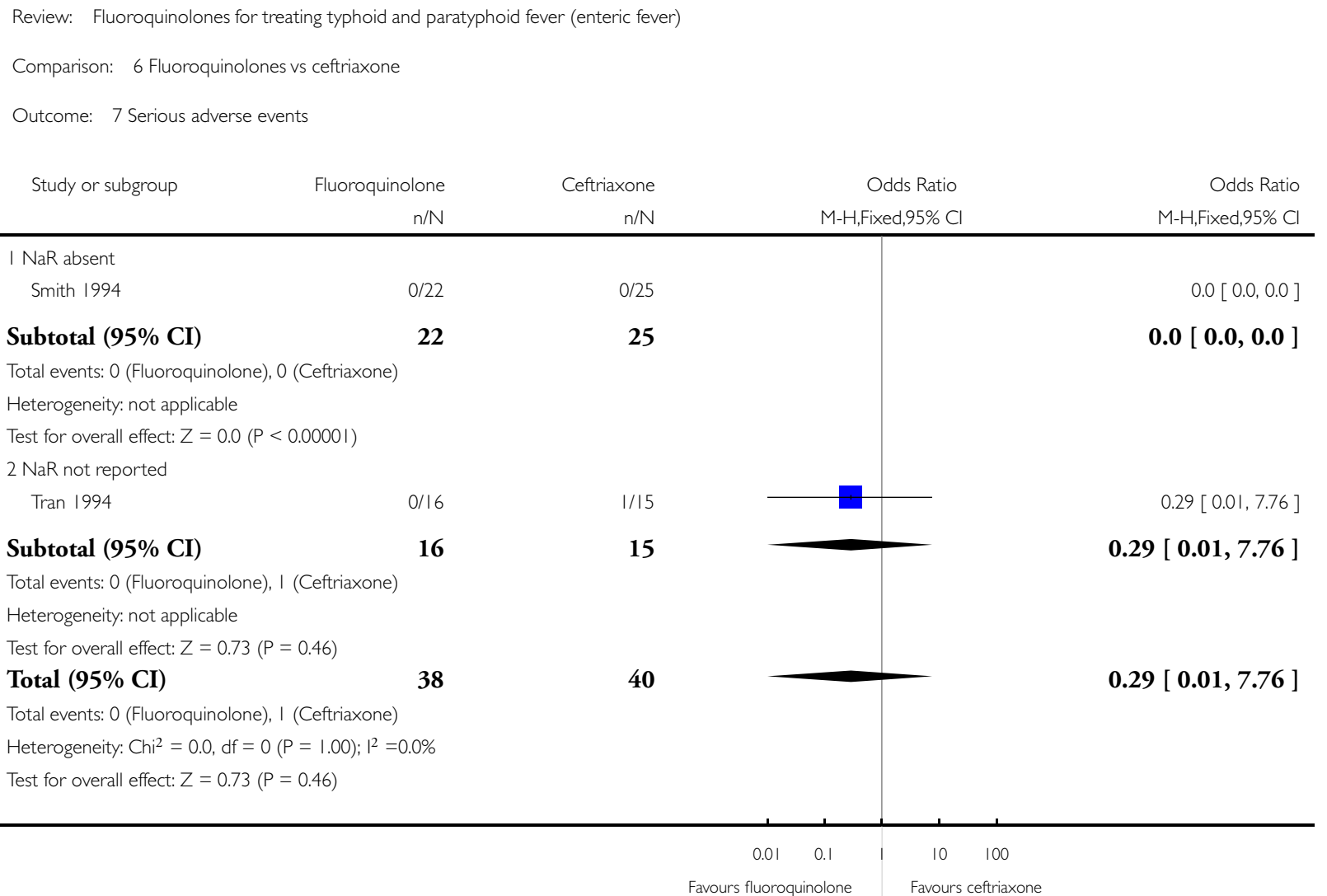

\section{Analysis 6.8. Comparison 6 Fluoroquinolones vs ceftriaxone, Outcome 8 Adverse events (not serious).}

Review: Fluoroquinolones for treating typhoid and paratyphoid fever (enteric fever)

Comparison: 6 Fluoroquinolones vs ceftriaxone

Outcome: 8 Adverse events (not serious)

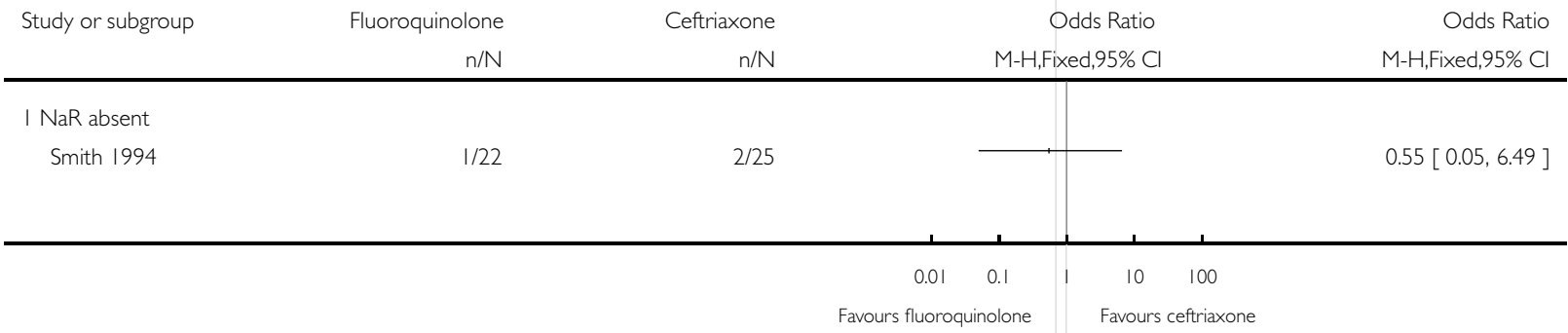




\section{Analysis 7.I. Comparison 7 Norfloxacin vs chloramphenicol, Outcome I Clinical failure.}

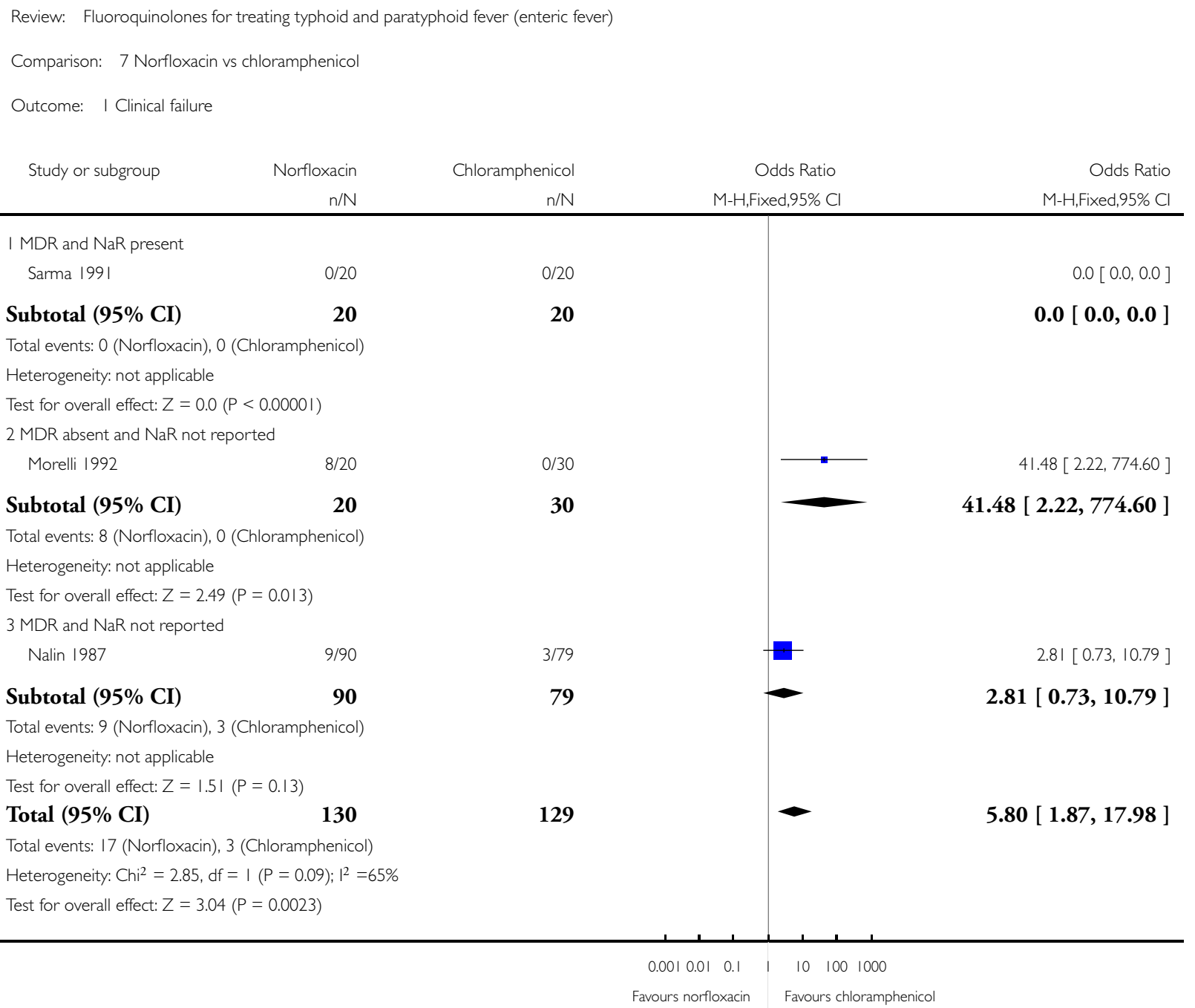




\section{Analysis 7.2. Comparison 7 Norfloxacin vs chloramphenicol, Outcome 2 Microbiological failure.}

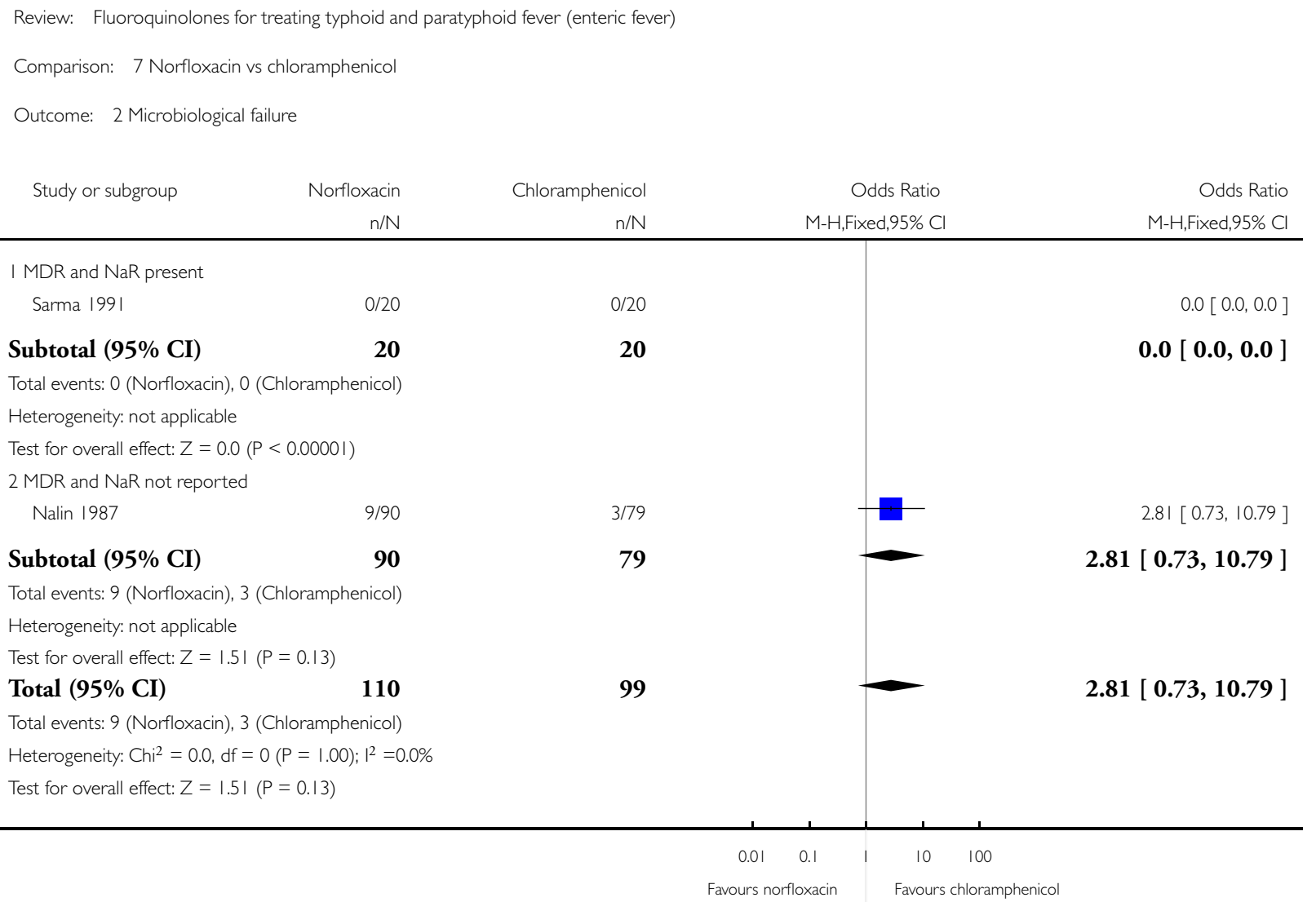




\section{Analysis 7.3. Comparison 7 Norfloxacin vs chloramphenicol, Outcome 3 Relapse.}

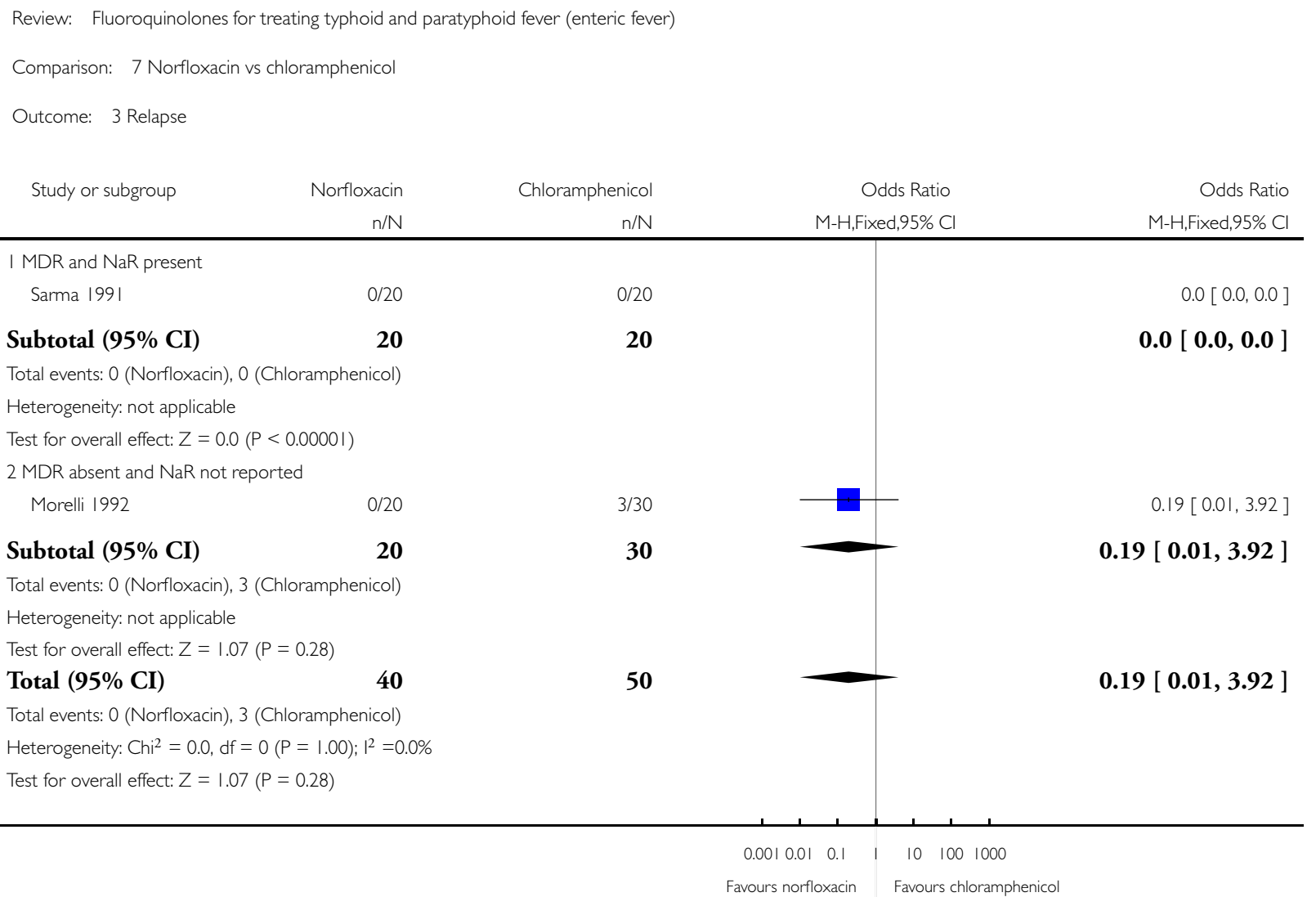

\section{Analysis 7.4. Comparison 7 Norfloxacin vs chloramphenicol, Outcome 4 Fever clearance time.}

Review: Fluoroquinolones for treating typhoid and paratyphoid fever (enteric fever)

Comparison: 7 Norfloxacin vs chloramphenicol

Outcome: 4 Fever clearance time

\begin{tabular}{|c|c|c|c|c|c|c|c|c|c|}
\hline \multirow[t]{2}{*}{ Study or subgroup } & Norfloxacin & \multicolumn{3}{|c|}{ Chloramphenicol } & & \multicolumn{3}{|c|}{ Mean Difference } & \multirow{2}{*}{$\begin{array}{l}\text { Mean Difference } \\
\text { IV,Fixed,95\% Cl }\end{array}$} \\
\hline & $\mathrm{N}$ & Mean(SD) & $\mathrm{N}$ & Mean(SD) & & $\mathrm{IV}, \mathrm{Fi}$ & $\mathrm{ed}, 95 \% \mathrm{Cl}$ & & \\
\hline \multicolumn{10}{|c|}{ I MDR and $\mathrm{NaR}$ present } \\
\hline Sarma |99| & 20 & $88(12)$ & 20 & $124(16)$ & & + & & & $-36.00[-44.77,-27.23]$ \\
\hline \multicolumn{10}{|c|}{$2 \mathrm{MDR}$ and $\mathrm{NaR}$ not reported } \\
\hline \multirow[t]{3}{*}{ Nalin 1987} & 90 & $160.8(60)$ & 79 & 122.4 (40.8) & & & 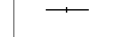 & & $38.40[23.08,53.72]$ \\
\hline & & & & & -100 & -50 & 50 & 100 & \\
\hline & & & & & \multicolumn{2}{|c|}{ Favours norfloxacin } & \multicolumn{3}{|c|}{ Favours chloramphenicol } \\
\hline
\end{tabular}




\section{Analysis 7.5. Comparison 7 Norfloxacin vs chloramphenicol, Outcome 5 Length of hospital stay (MDR and}

NaR present).

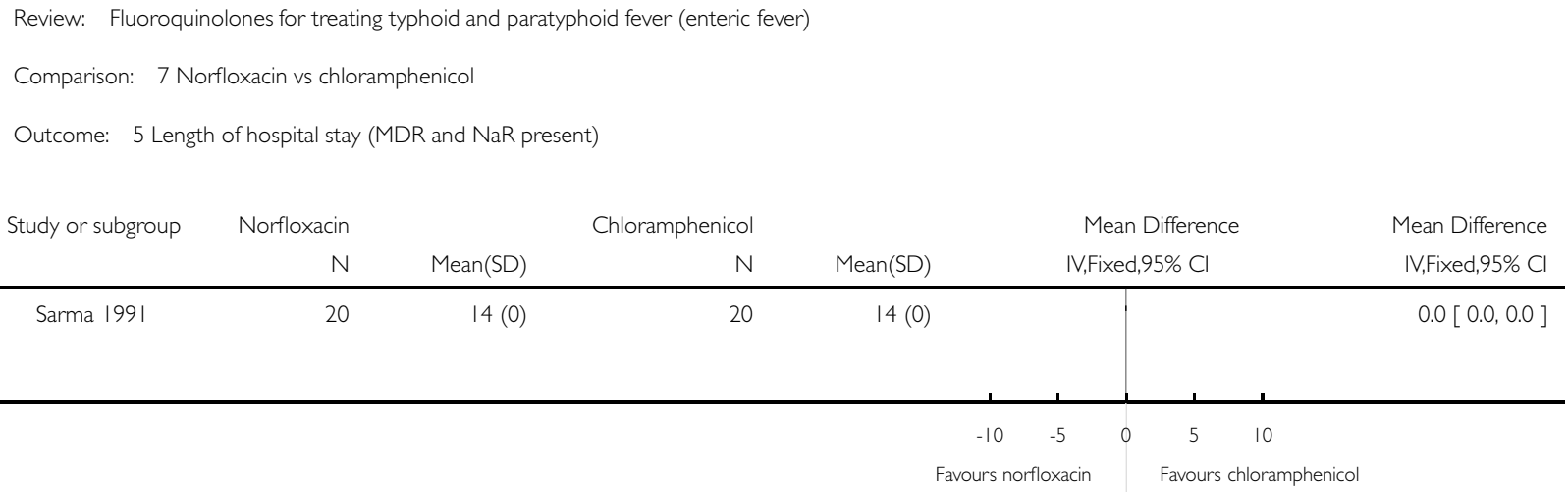

Analysis 7.6. Comparison 7 Norfloxacin vs chloramphenicol, Outcome 6 Convalescent faecal carriage.

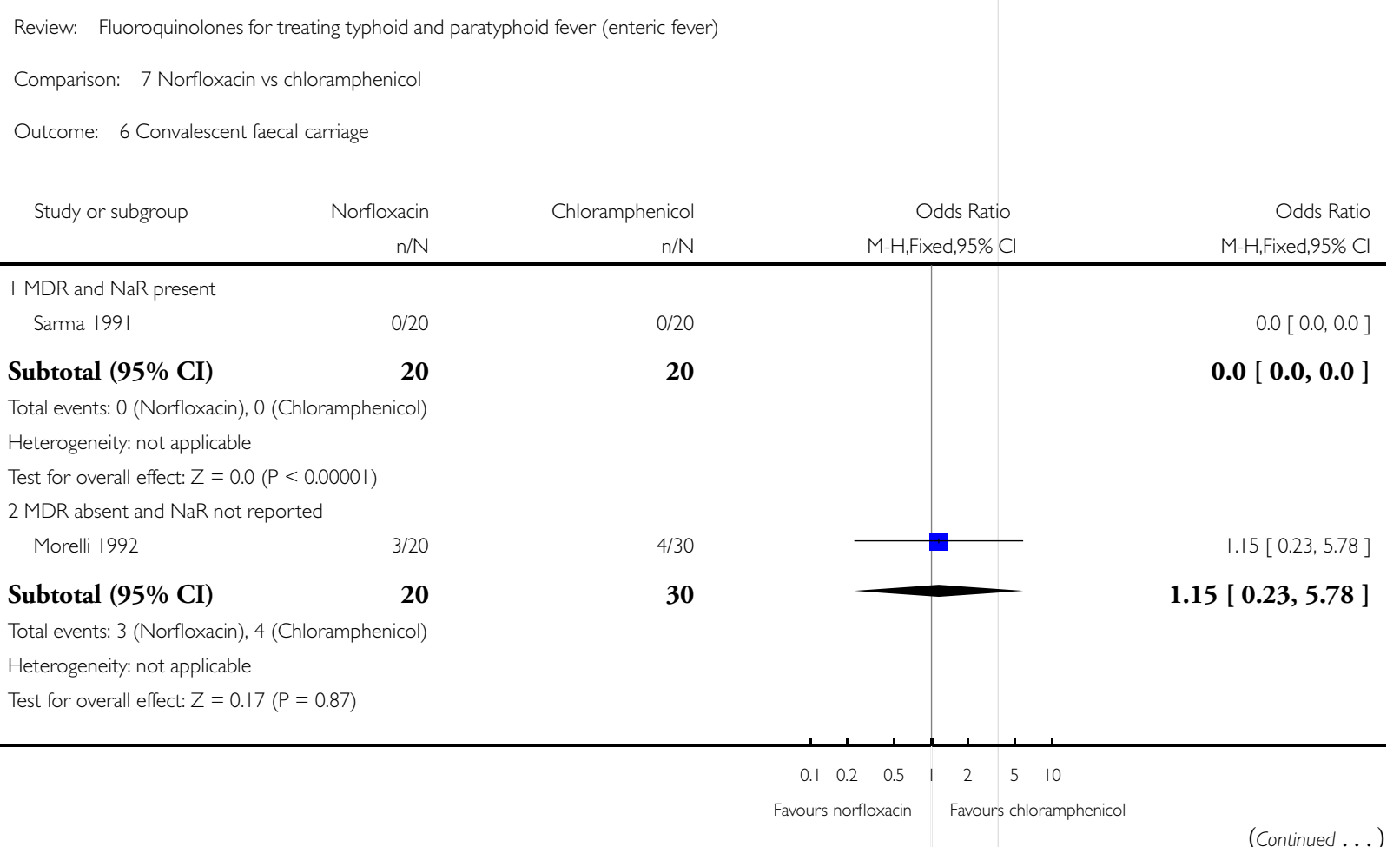




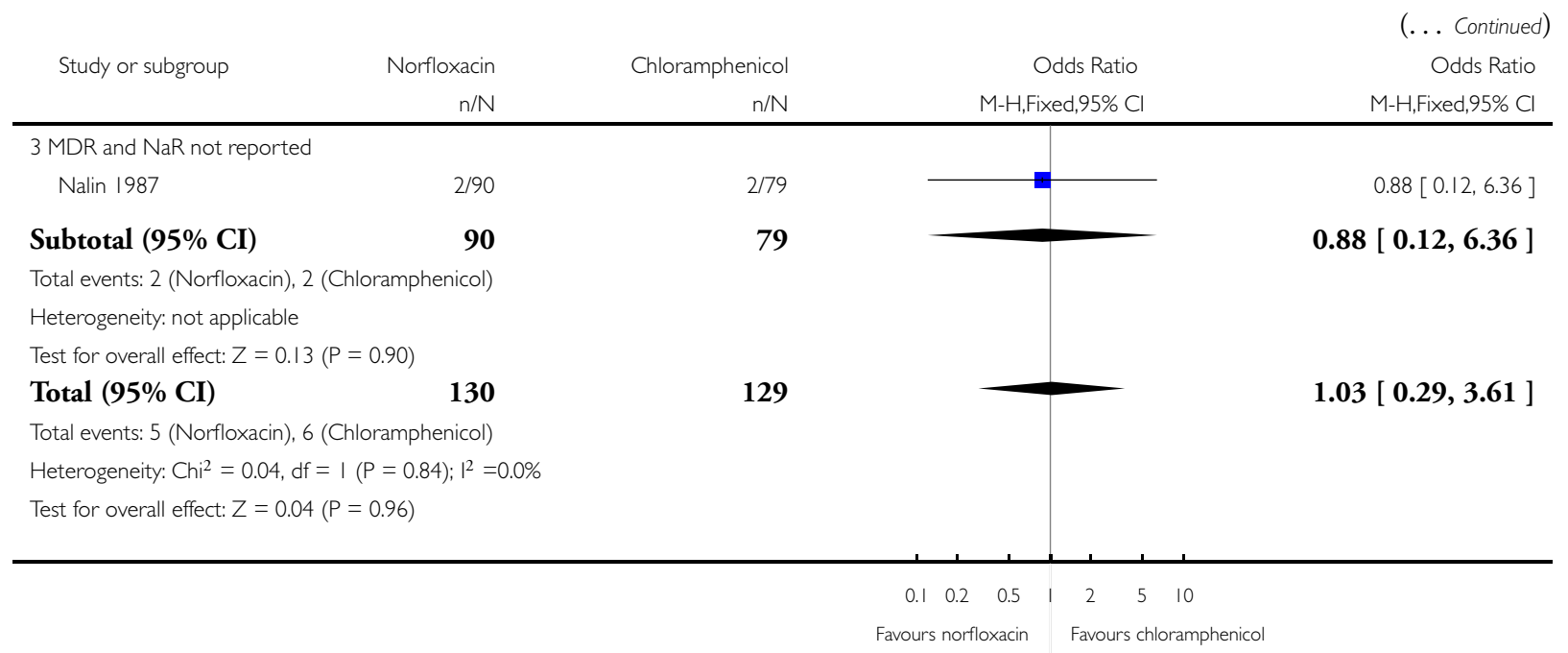

\section{Analysis 7.7. Comparison 7 Norfloxacin vs chloramphenicol, Outcome 7 Adverse events (not serious).}

Review: Fluoroquinolones for treating typhoid and paratyphoid fever (enteric fever)

Comparison: 7 Norfloxacin vs chloramphenicol

Outcome: 7 Adverse events (not serious)

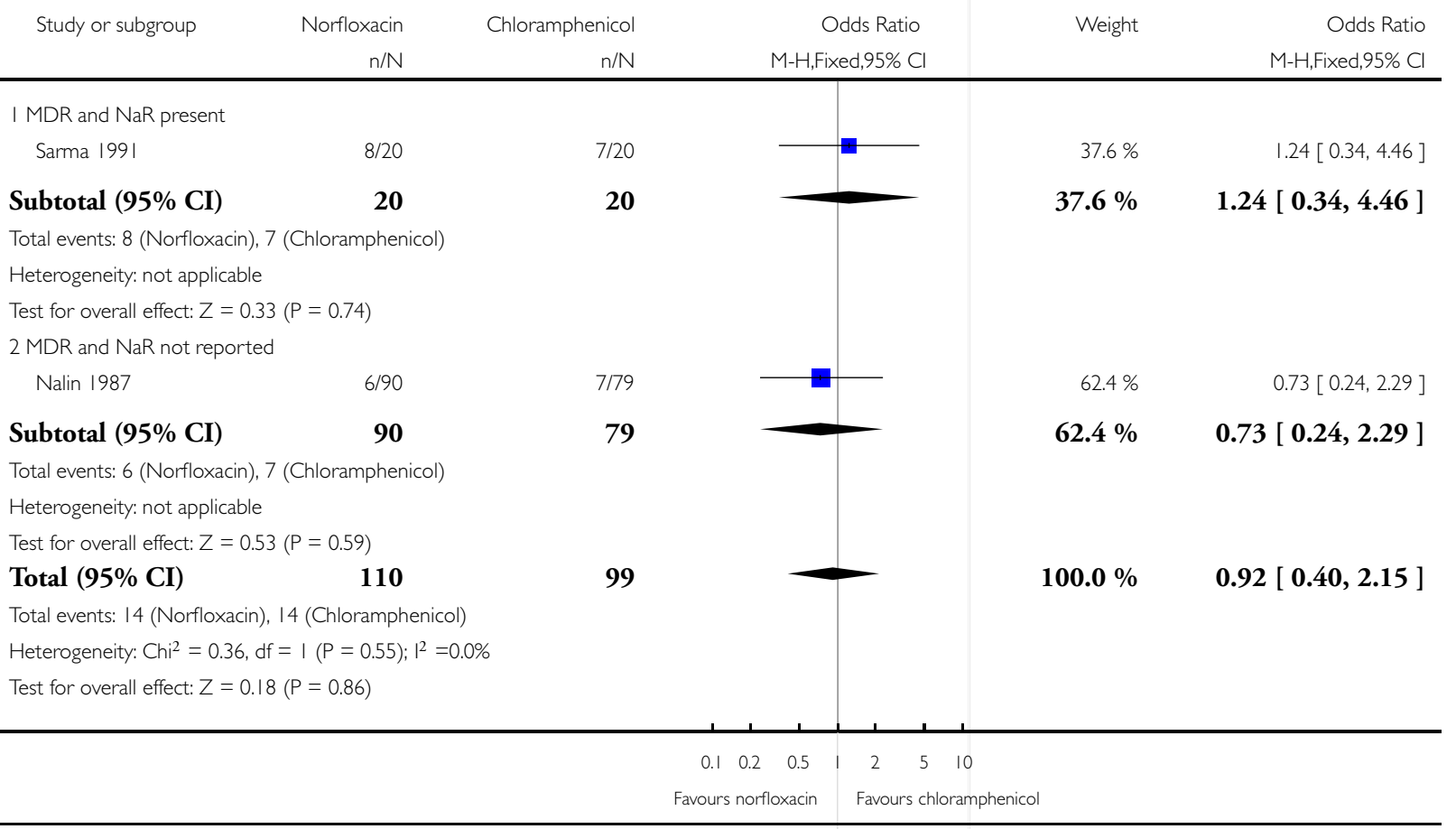




\section{Analysis 8.I. Comparison 8 Norfloxacin vs ceftriaxone, Outcome I Clinical failure (NaR not reported).}

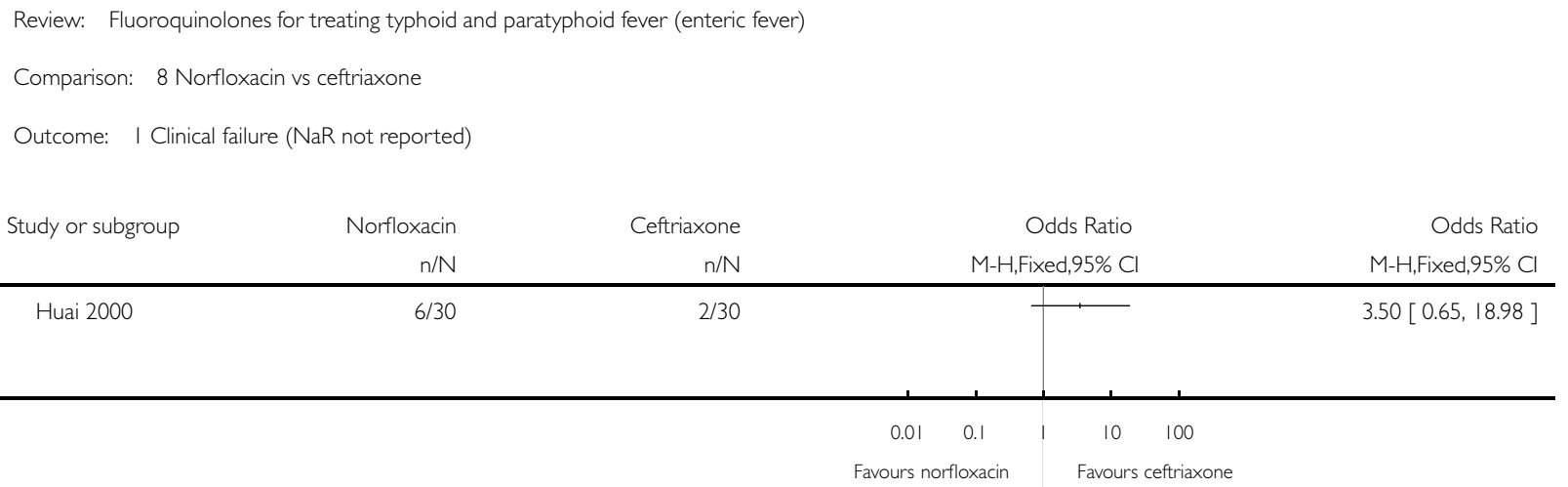

Analysis 8.2. Comparison 8 Norfloxacin vs ceftriaxone, Outcome 2 Relapse (NaR not reported).

\begin{tabular}{|c|c|c|c|c|}
\hline \multicolumn{5}{|c|}{ Comparison: 8 Norfloxacin vs ceftriaxone } \\
\hline \multicolumn{5}{|c|}{ Outcome: 2 Relapse (NaR not reported) } \\
\hline \multirow[t]{2}{*}{ Study or subgroup } & Norfloxacin & Ceftriaxone & Odds Ratio & Odds Ratio \\
\hline & $\mathrm{n} / \mathrm{N}$ & $n / N$ & M-H,Fixed,95\% Cl & M-H,Fixed,95\% Cl \\
\hline Huai 2000 & 2/30 & 0/30 & $\longrightarrow$ & $5.35[0.25,116.31]$ \\
\hline
\end{tabular}


Analysis 8.3. Comparison 8 Norfloxacin vs ceftriaxone, Outcome 3 Fever clearance time (NaR not reported).

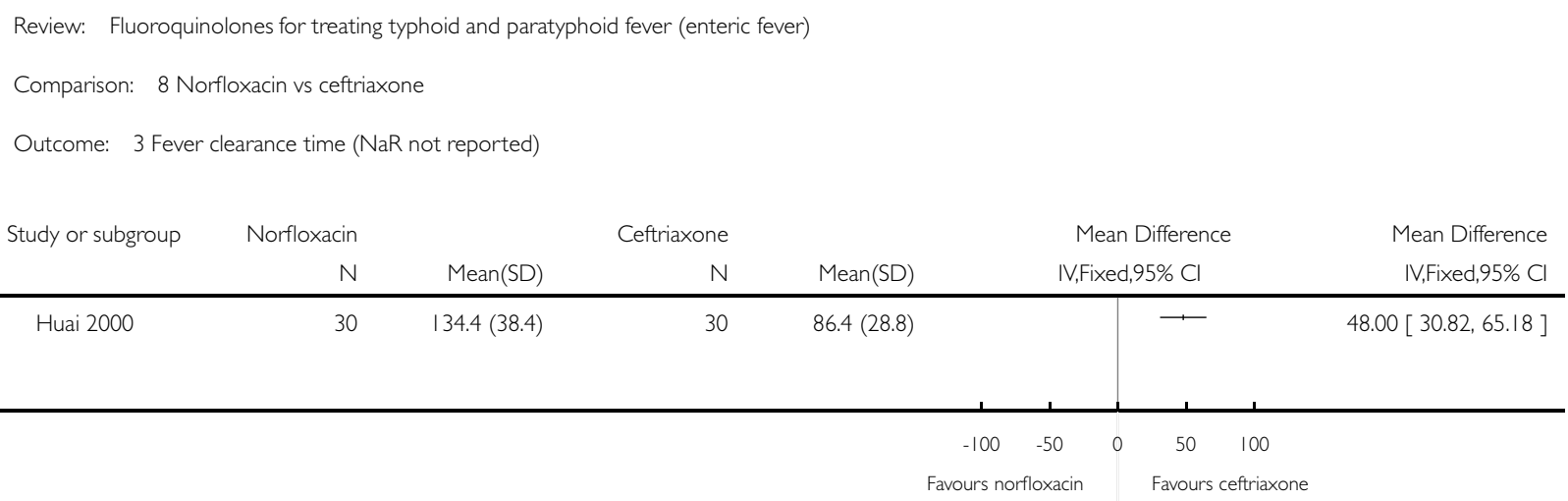

Analysis 8.4. Comparison 8 Norfloxacin vs ceftriaxone, Outcome 4 Adverse events (not serious) (NaR not reported).

Review: Fluoroquinolones for treating typhoid and paratyphoid fever (enteric fever)

Comparison: 8 Norfloxacin vs ceftriaxone

Outcome: 4 Adverse events (not serious) (NaR not reported)

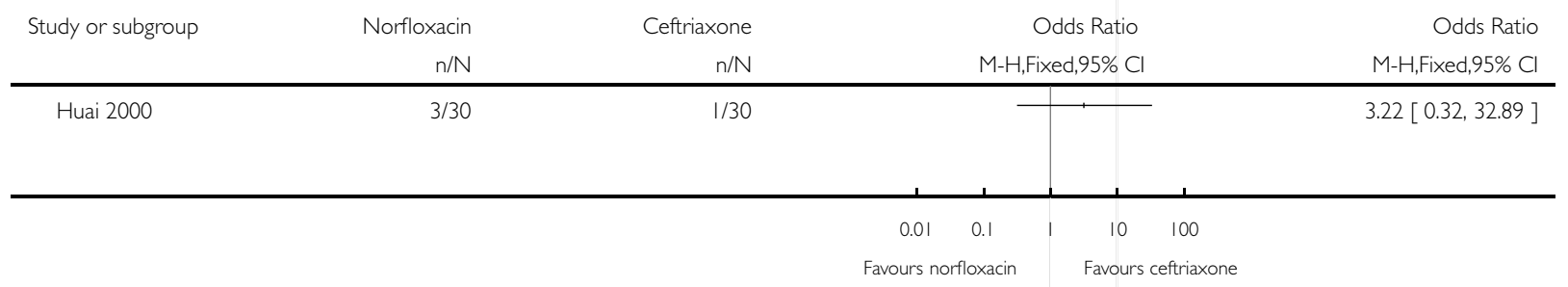




\section{Analysis 9.1. Comparison 9 Norfloxacin vs other fluoroquinolones (FQ), Outcome I Clinical failure.}

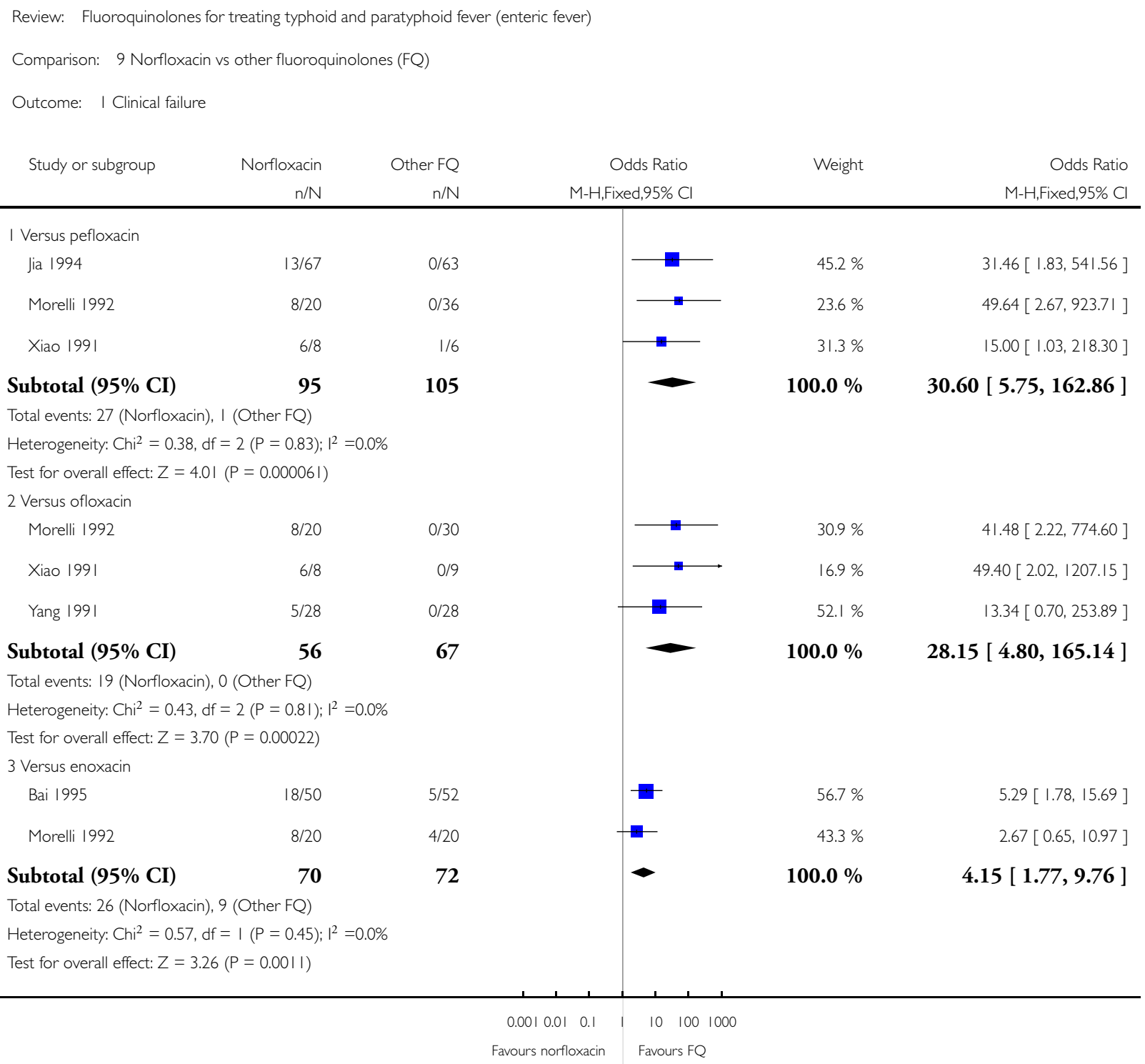




\section{Analysis 9.2. Comparison 9 Norfloxacin vs other fluoroquinolones (FQ), Outcome 2 Relapse.}

Review: Fluoroquinolones for treating typhoid and paratyphoid fever (enteric fever)

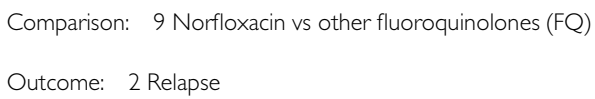

\section{Analysis 9.3. Comparison 9 Norfloxacin vs other fluoroquinolones (FQ), Outcome 3 Fever clearance time.}

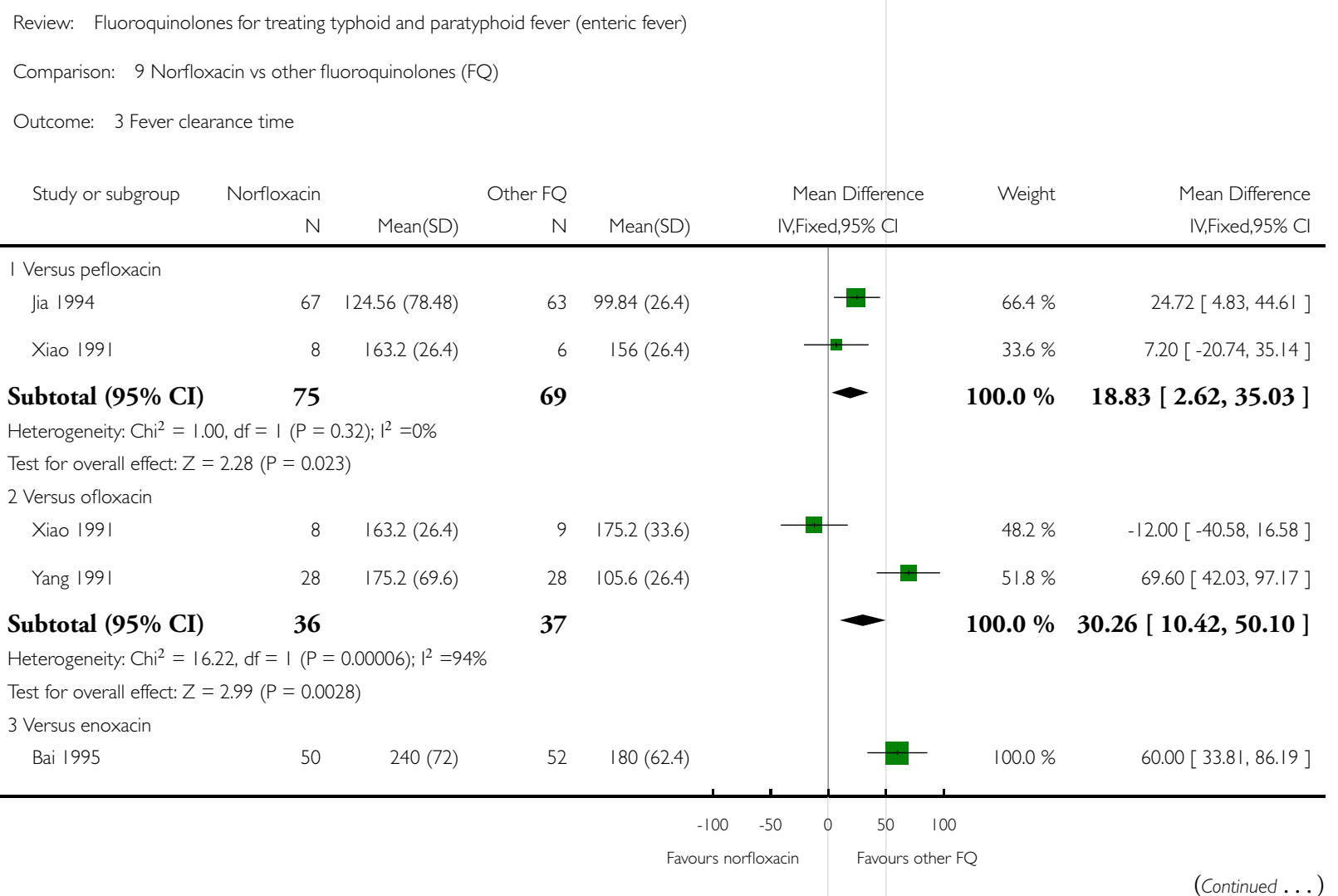




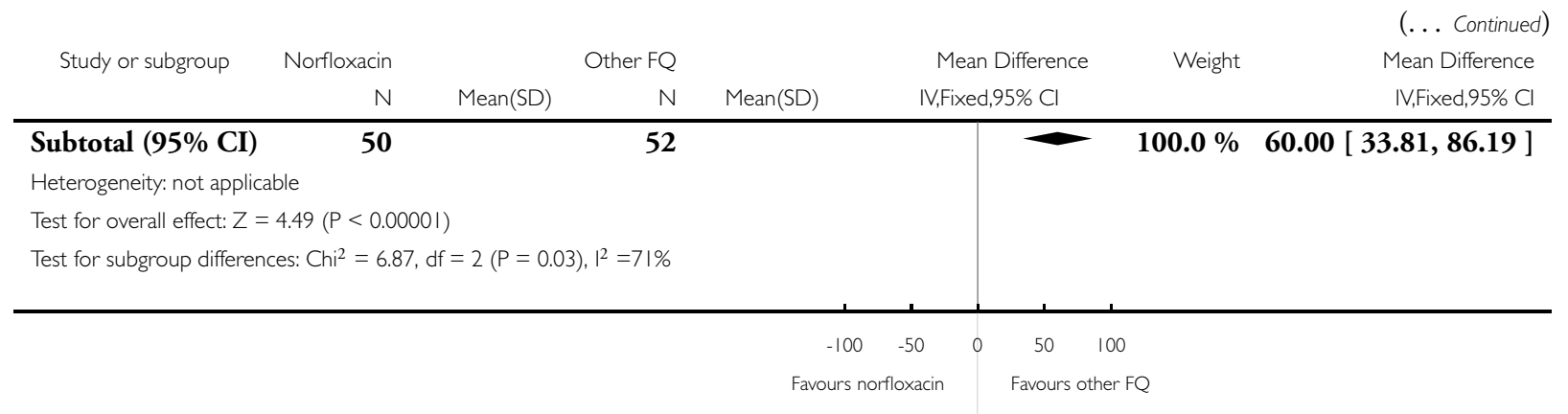

Analysis 9.4. Comparison 9 Norfloxacin vs other fluoroquinolones (FQ), Outcome 4 Convalescent faecal carriage.

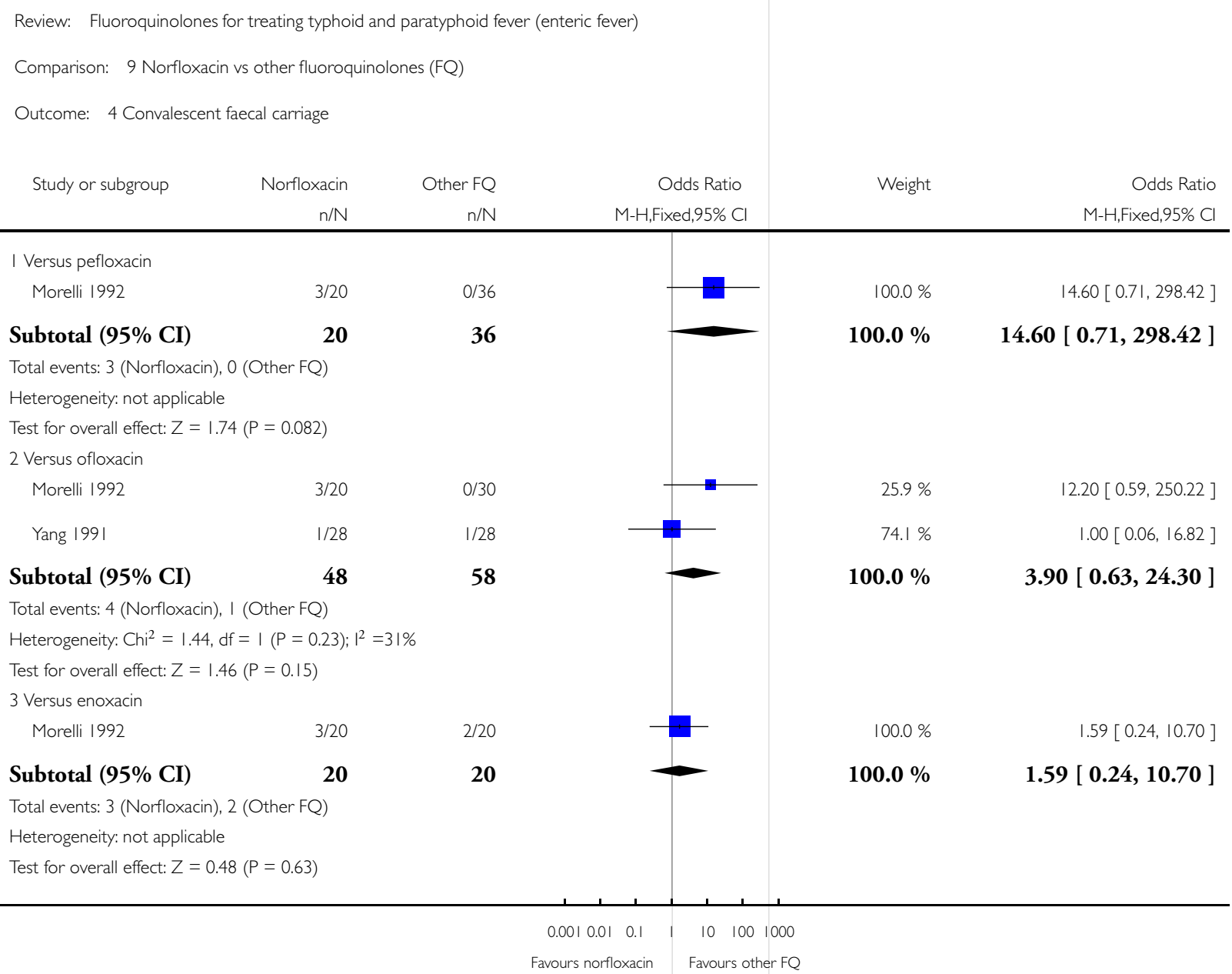


Analysis 9.5. Comparison 9 Norfloxacin vs other fluoroquinolones (FQ), Outcome 5 Adverse events (not serious).

\begin{tabular}{|c|c|c|c|c|}
\hline \multicolumn{5}{|c|}{ Comparison: 9 Norfloxacin vs other fluoroquinolones (FQ) } \\
\hline \multicolumn{5}{|c|}{ Outcome: 5 Adverse events (not serious) } \\
\hline \multirow[t]{2}{*}{ Study or subgroup } & Norfloxacin & Other FQ & Odds Ratio & Odds Ratio \\
\hline & $\mathrm{n} / \mathrm{N}$ & $\mathrm{n} / \mathrm{N}$ & M-H,Fixed, $95 \% \mathrm{Cl}$ & M-H,Fixed, $95 \% \mathrm{Cl}$ \\
\hline \multicolumn{5}{|l|}{ I Versus ofloxacin } \\
\hline Yang 199| & 1/28 & $1 / 28$ & 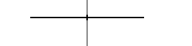 & $1.00[0.06,16.82]$ \\
\hline \multicolumn{5}{|l|}{2 Versus enoxacin } \\
\hline Bai 1995 & 1/50 & 1/52 & 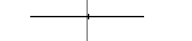 & $1.04[0.06,17.11]$ \\
\hline
\end{tabular}

Analysis 10.I. Comparison 10 Fluoroquinolones for 2 days vs 3 days, Outcome I Clinical failure ( $\mathrm{NaR}$ present).

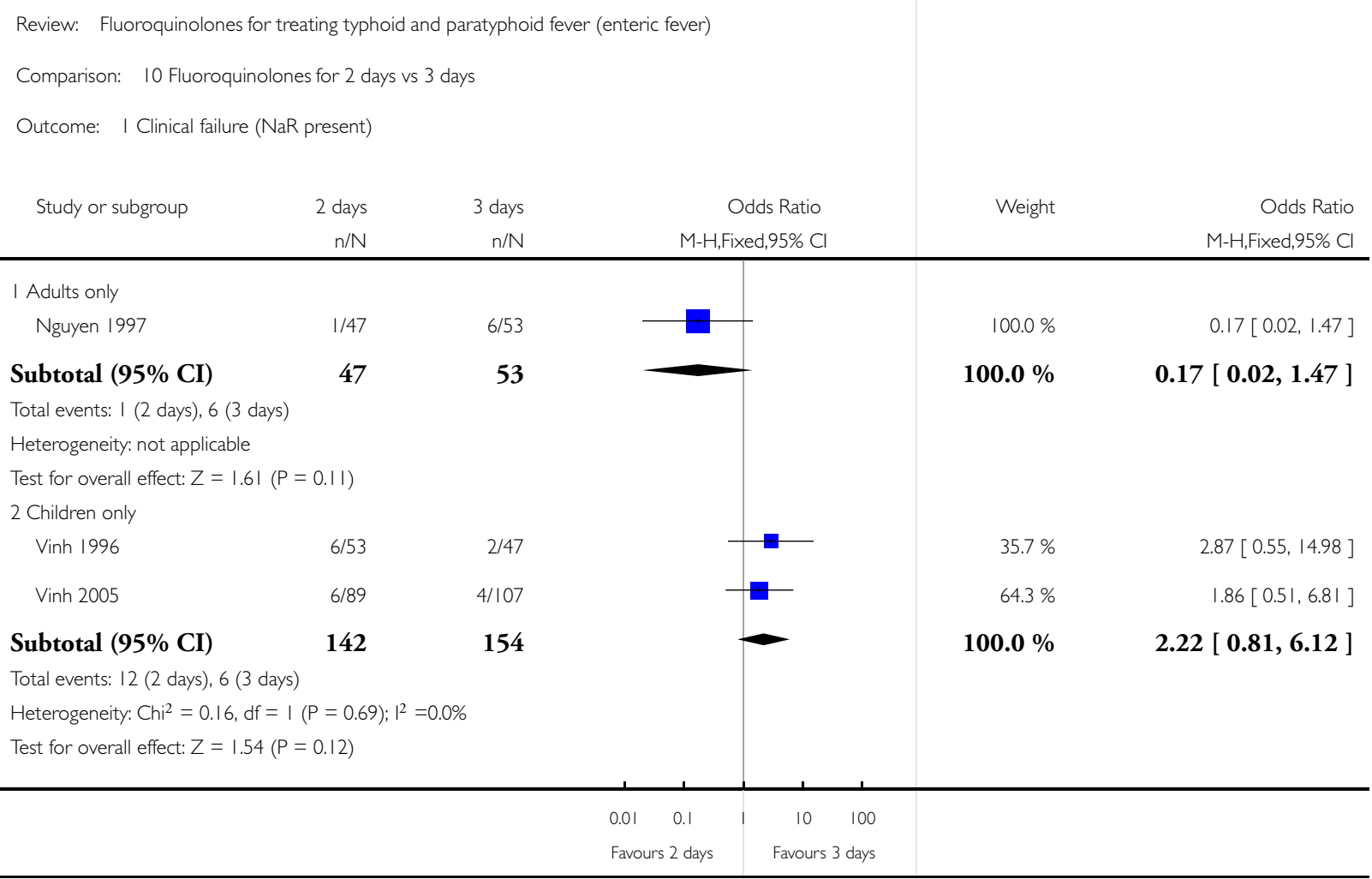


Analysis 10.2. Comparison 10 Fluoroquinolones for 2 days vs 3 days, Outcome 2 Microbiological failure (NaR present).

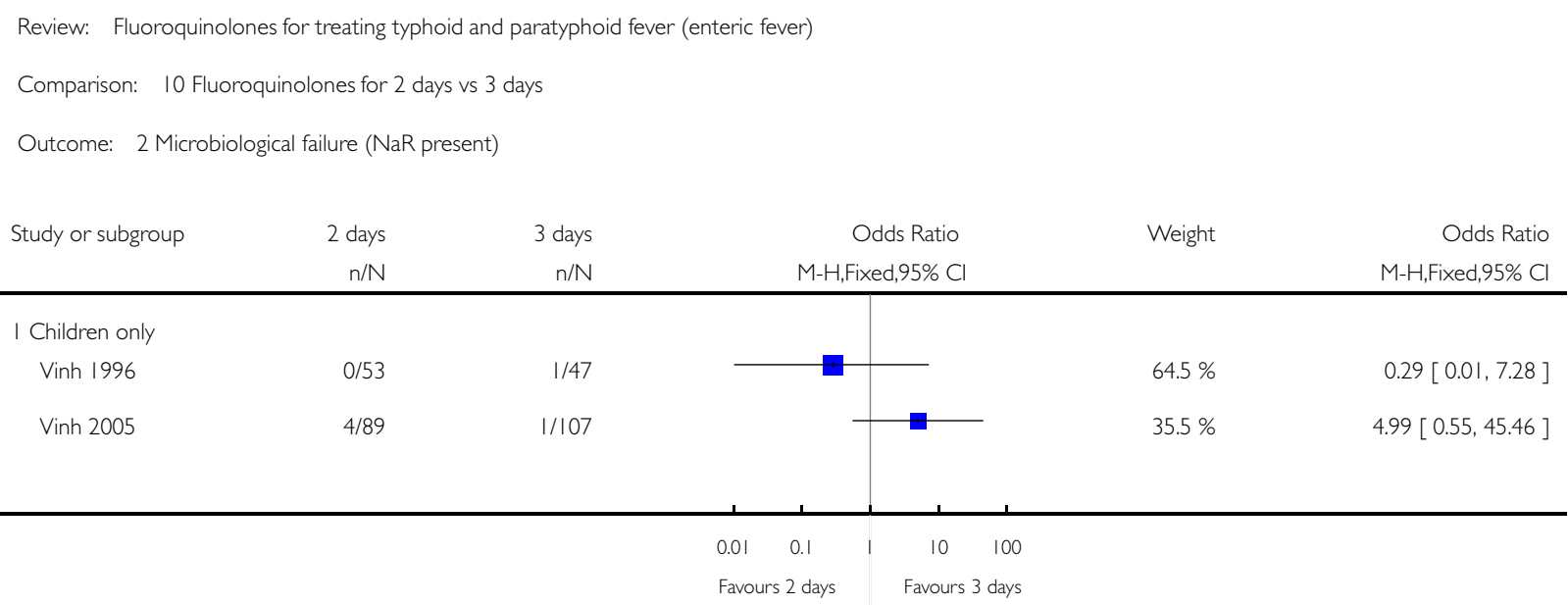


Analysis 10.3. Comparison 10 Fluoroquinolones for 2 days vs 3 days, Outcome 3 Relapse (NaR present).

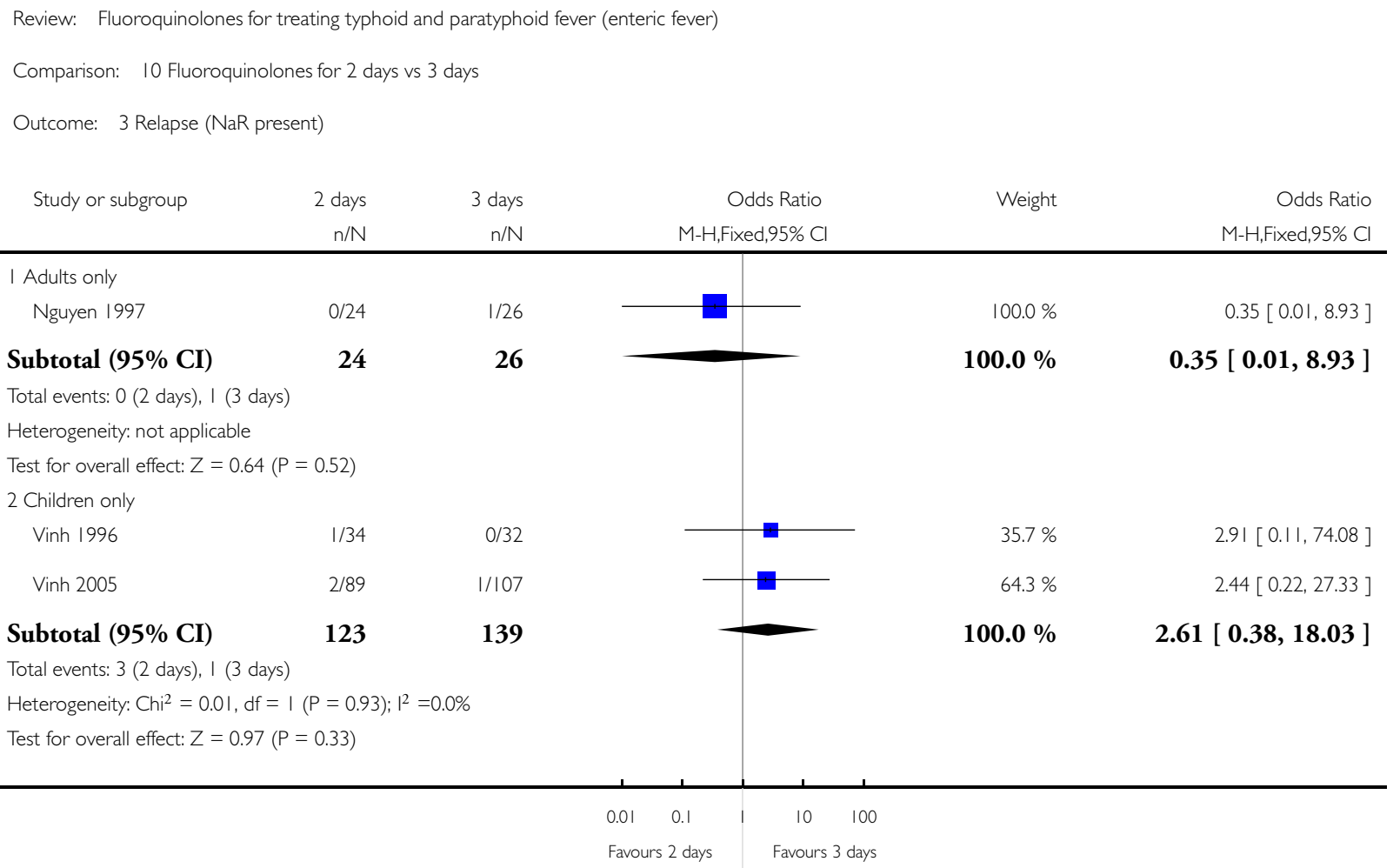


Analysis 10.4. Comparison 10 Fluoroquinolones for 2 days vs 3 days, Outcome 4 Fever clearance time (NaR present).

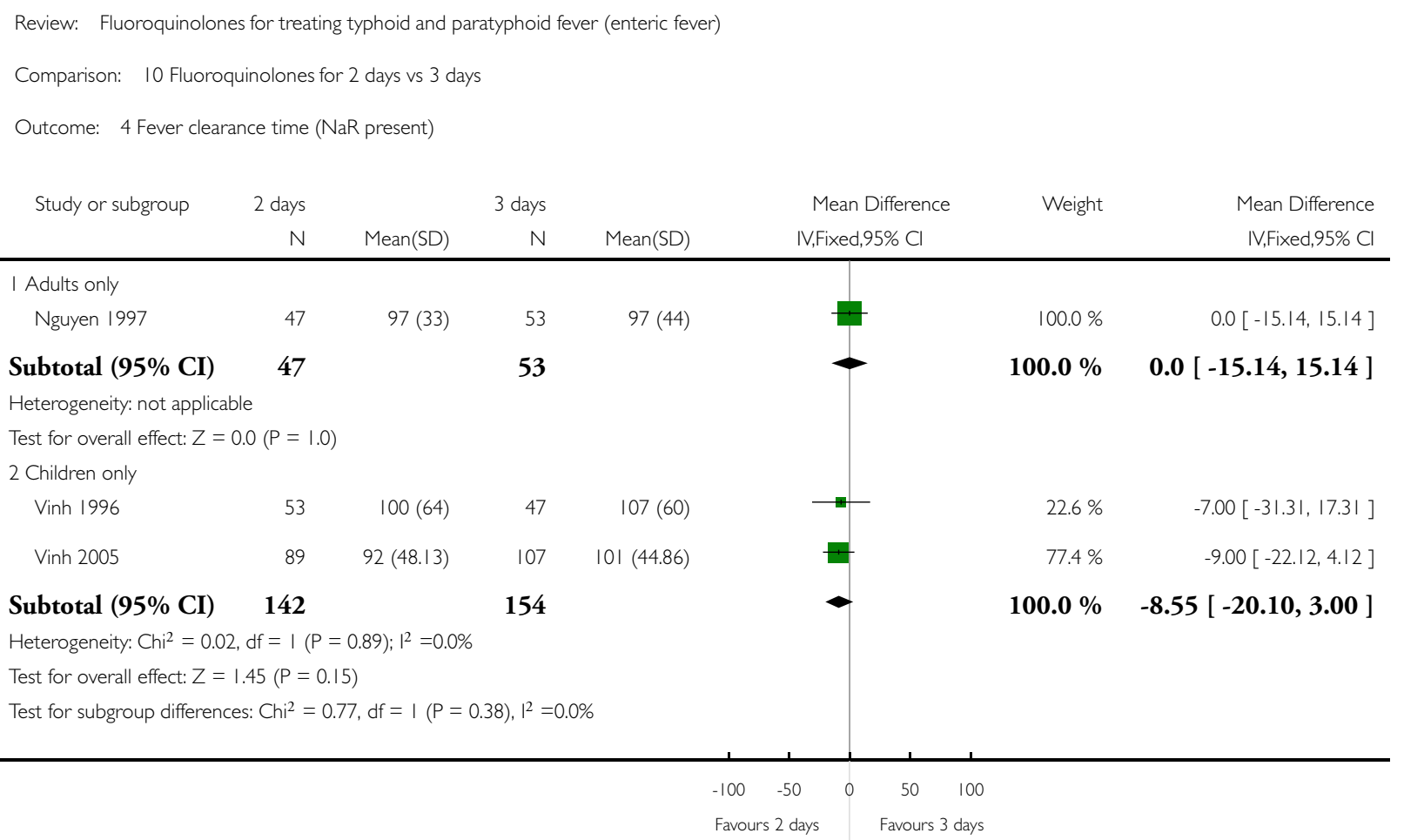


Analysis 10.5. Comparison 10 Fluoroquinolones for 2 days vs 3 days, Outcome 5 Length of hospital stay (NaR present).

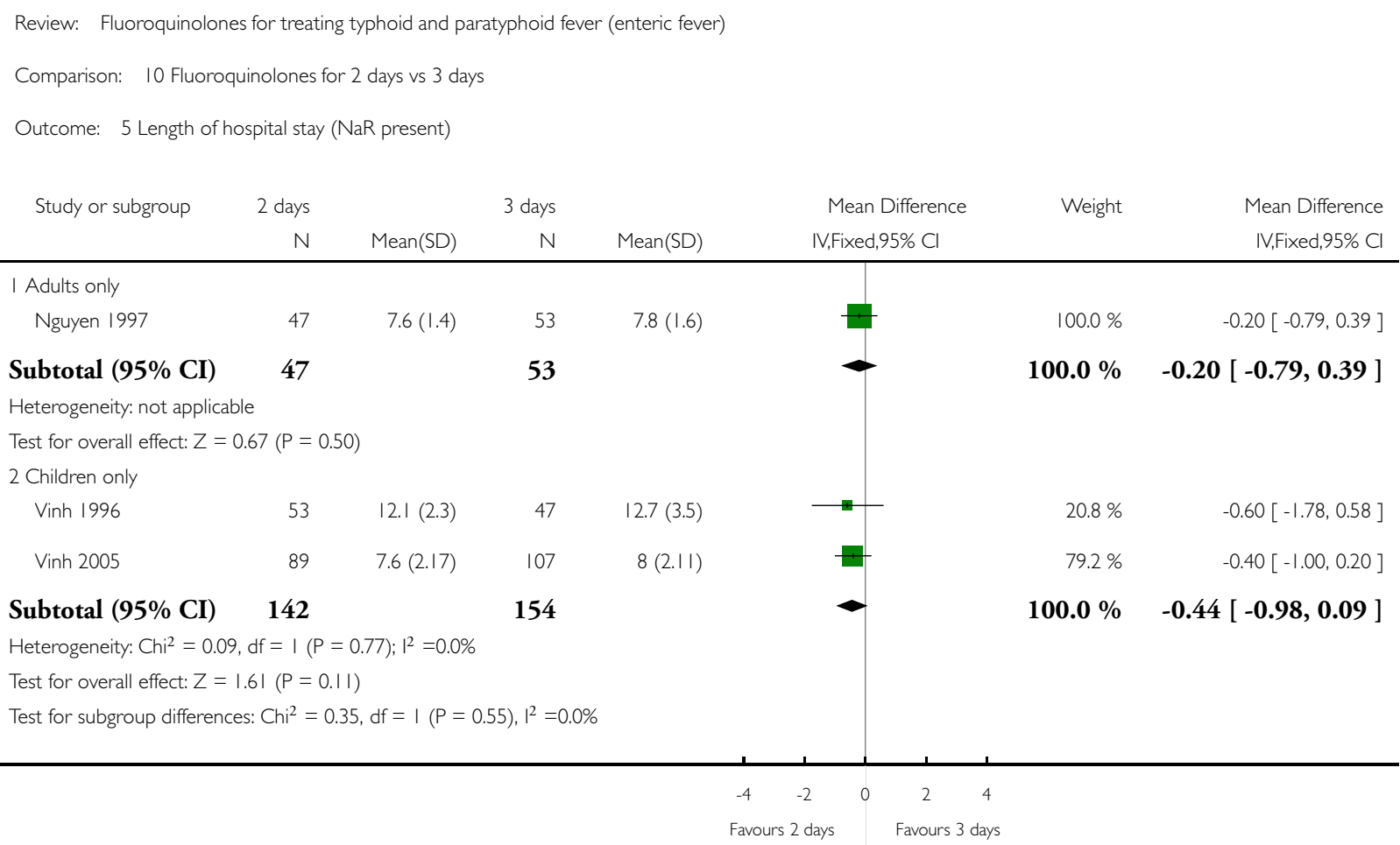

\section{Analysis 10.6. Comparison I0 Fluoroquinolones for 2 days vs 3 days, Outcome 6 Convalescent faecal} carriage (NaR present).

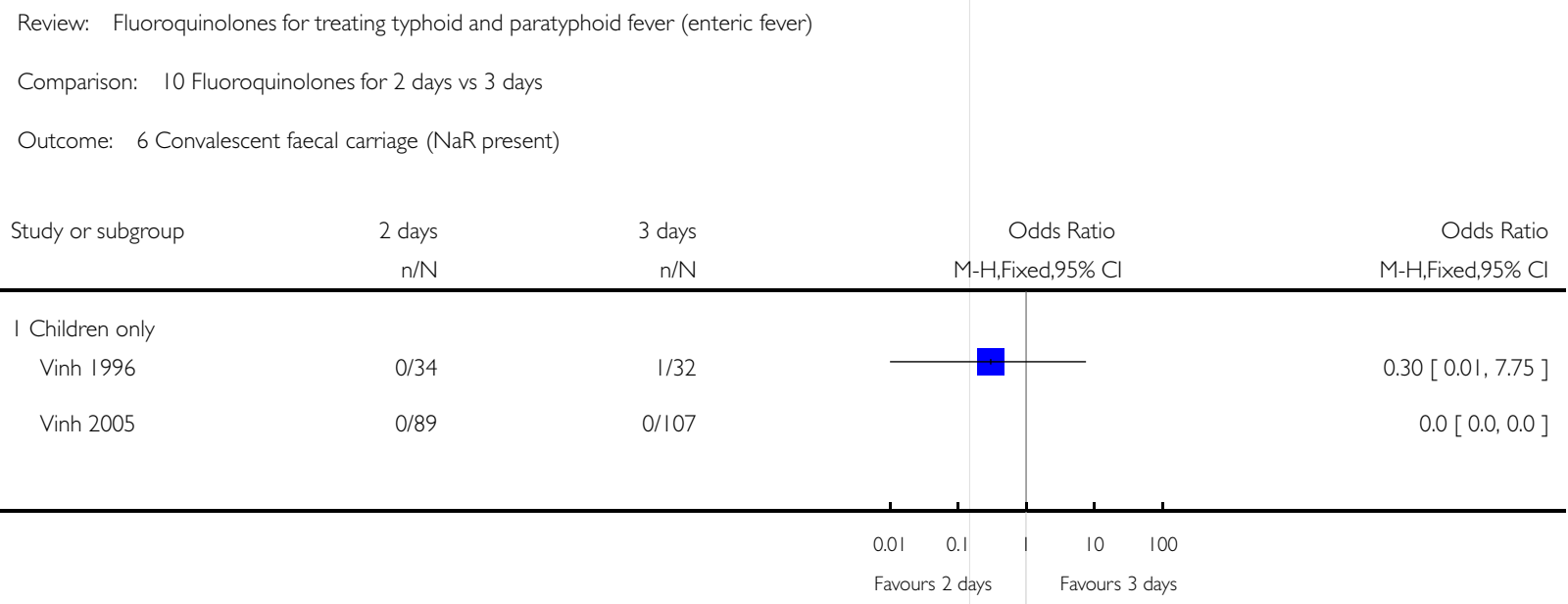


Analysis 10.7. Comparison 10 Fluoroquinolones for 2 days vs 3 days, Outcome 7 Complications (NaR present).

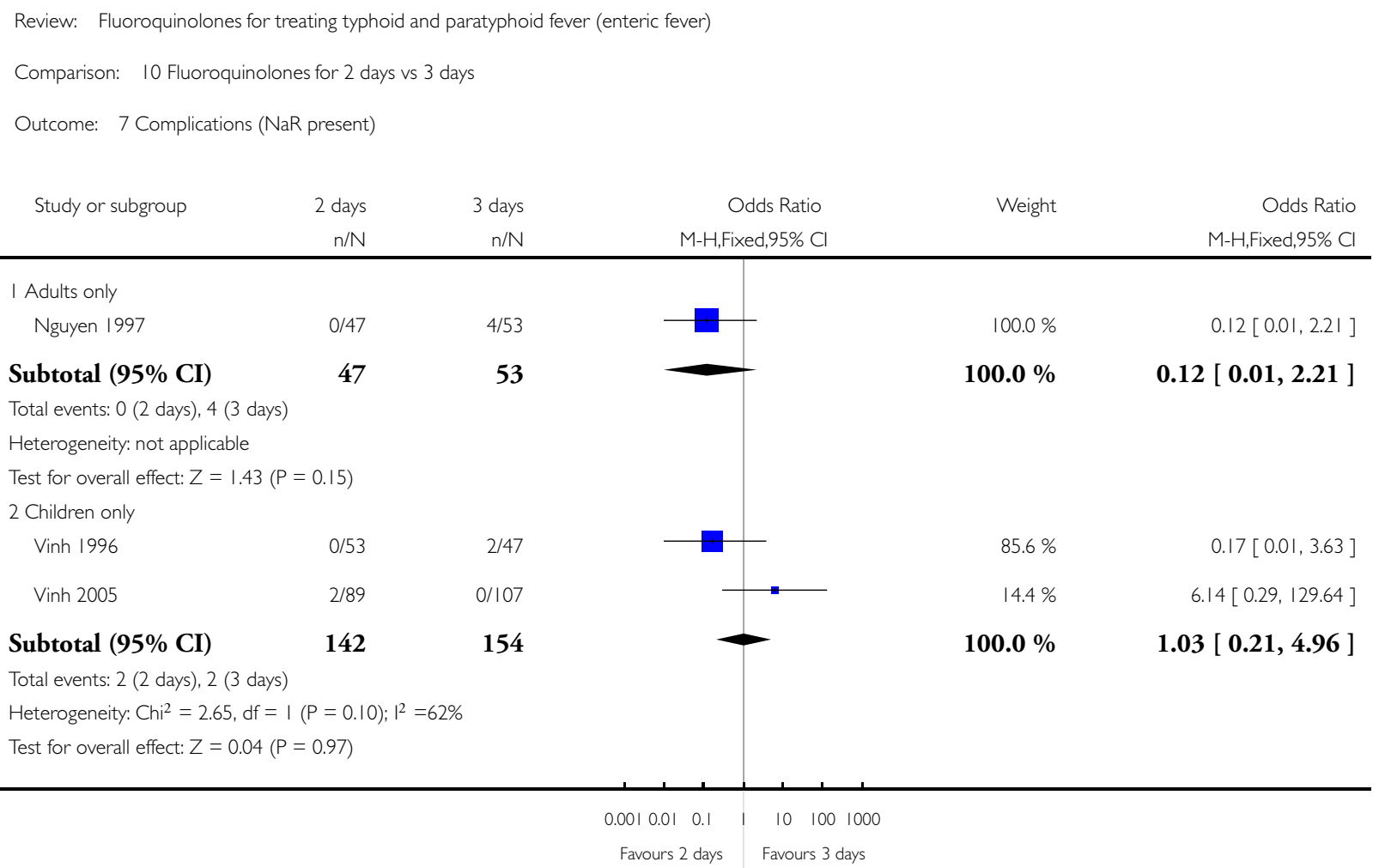

\section{Analysis 10.8. Comparison 10 Fluoroquinolones for 2 days vs 3 days, Outcome 8 Adverse events (not serious) (NaR present).}

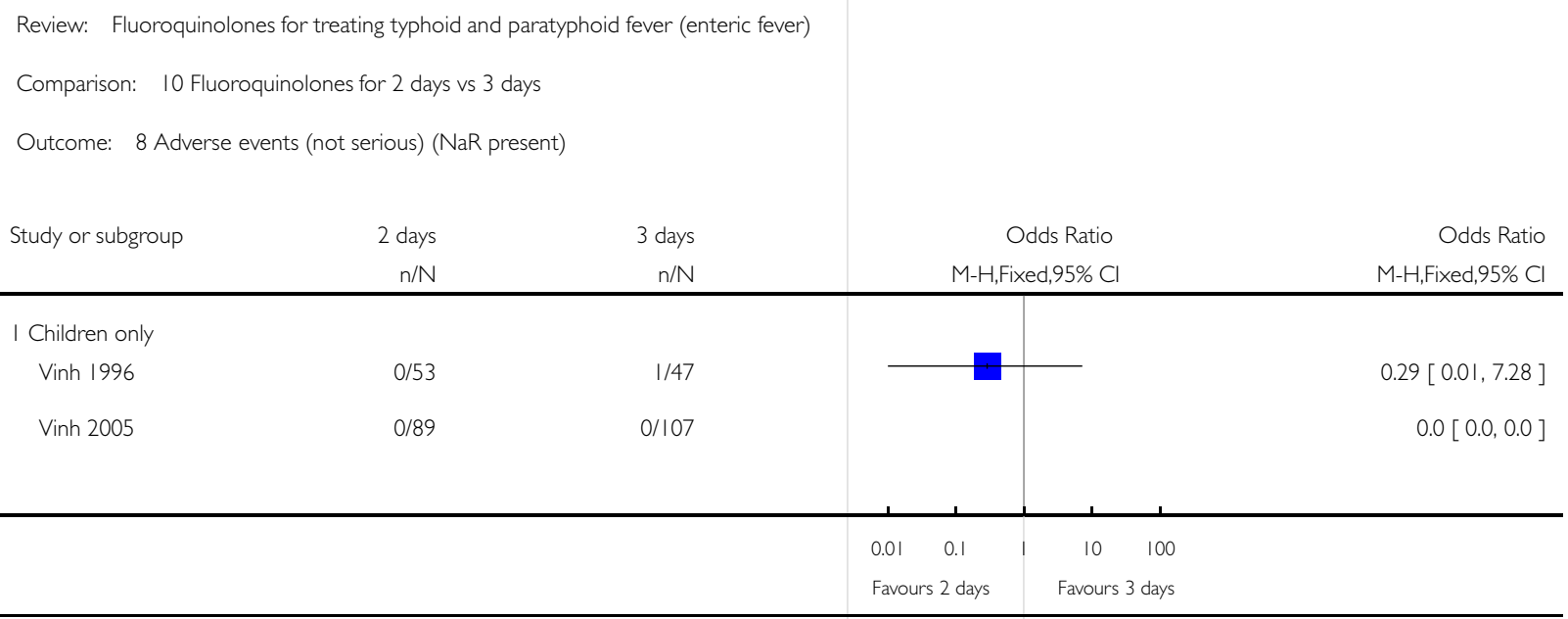


Analysis I I.I. Comparison I I Fluoroquinolones for 3 days vs 5 days, Outcome I Clinical failure (NaR not reported).

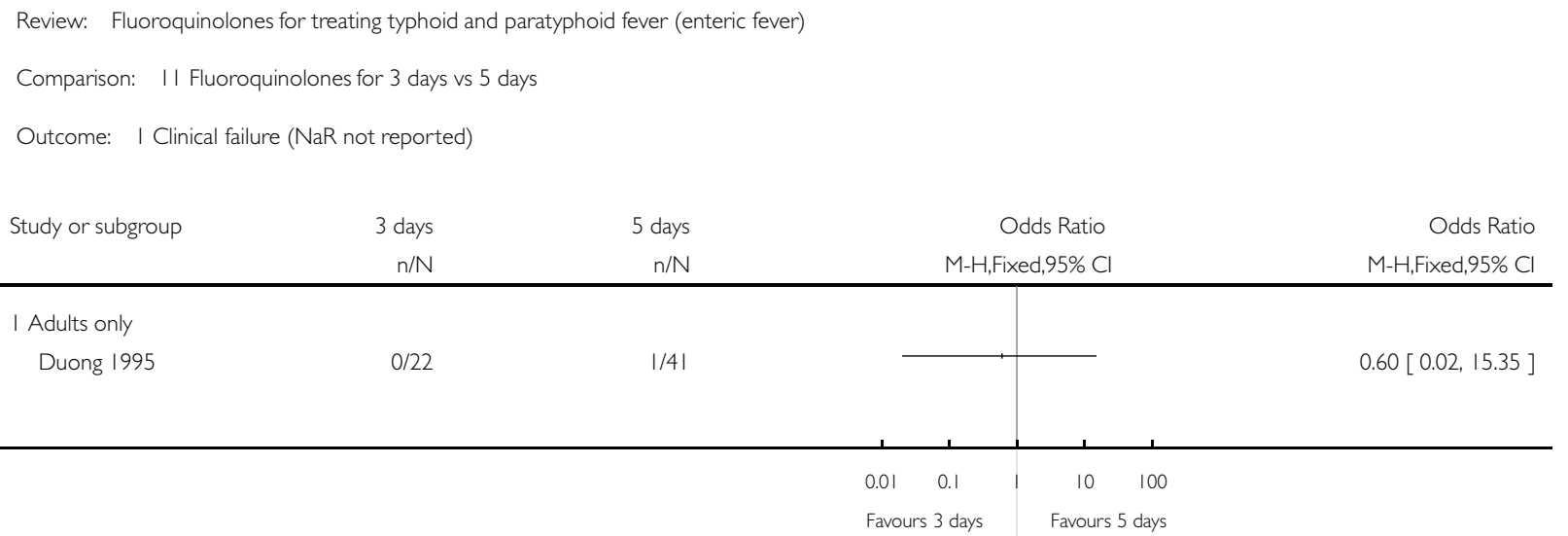

\section{Analysis I I.2. Comparison I I Fluoroquinolones for 3 days vs 5 days, Outcome 2 Relapse.}

Review: Fluoroquinolones for treating typhoid and paratyphoid fever (enteric fever)

Comparison: II Fluoroquinolones for 3 days vs 5 days

Outcome: 2 Relapse

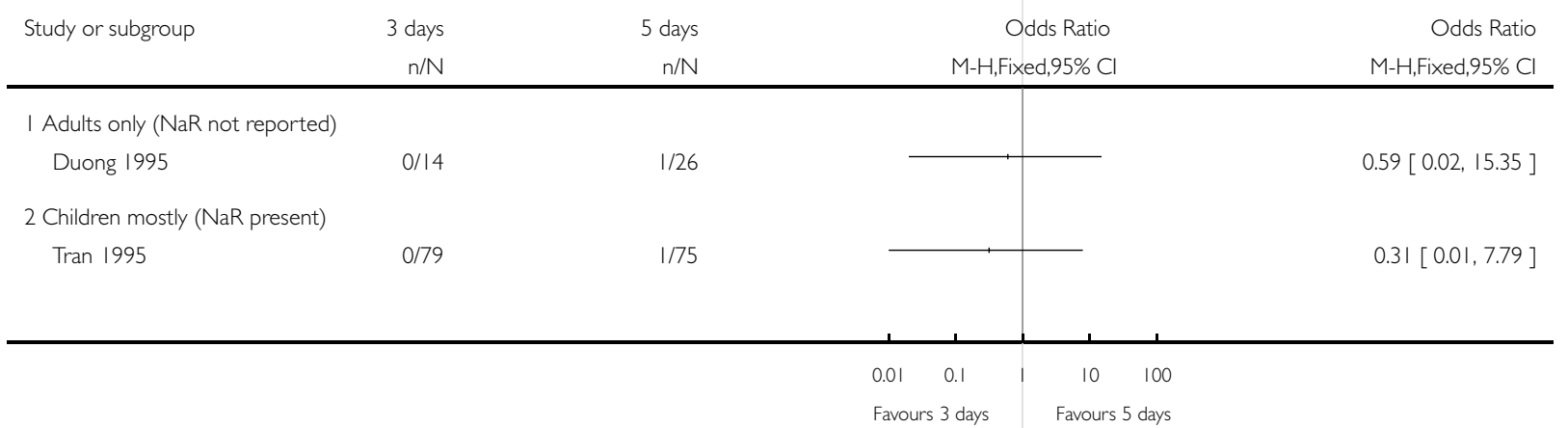


Analysis II.3. Comparison I I Fluoroquinolones for 3 days vs 5 days, Outcome 3 Fever clearance time.

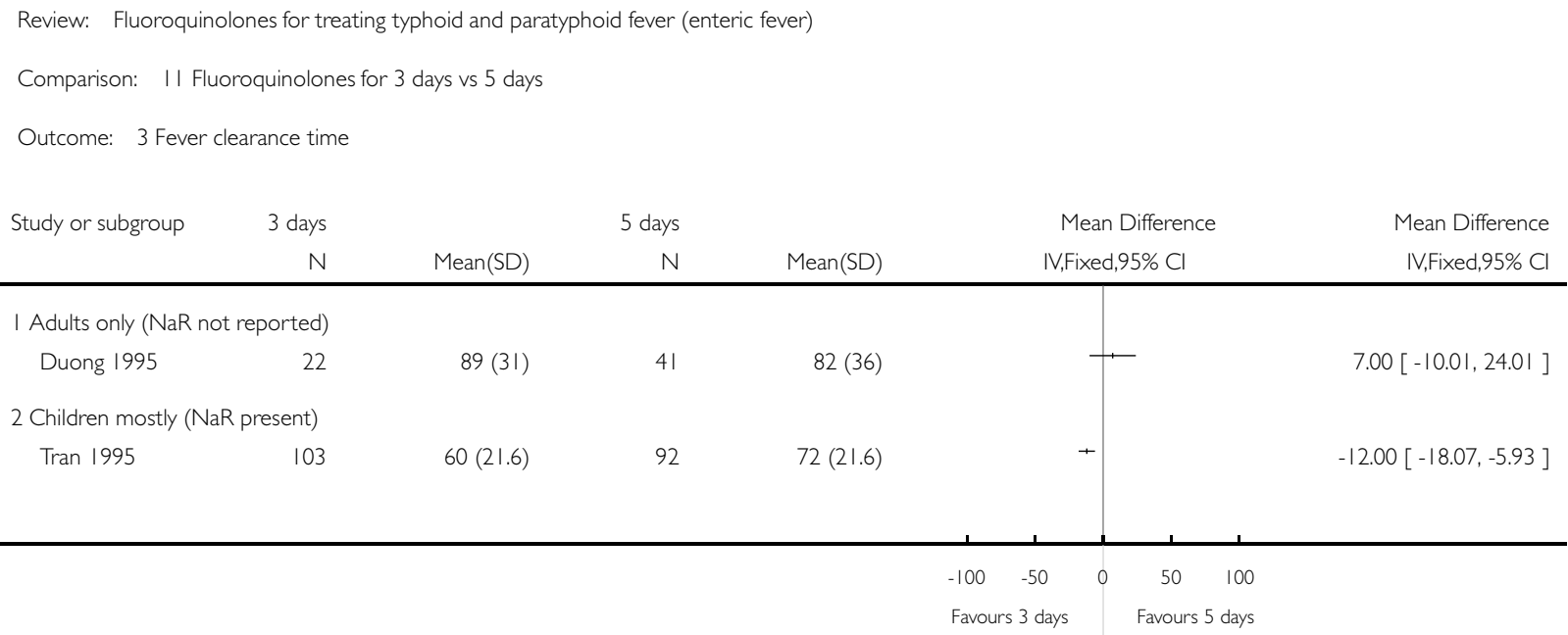

\section{Analysis I I.4. Comparison II Fluoroquinolones for 3 days vs 5 days, Outcome 4 Length of hospital stay} (NaR not reported).

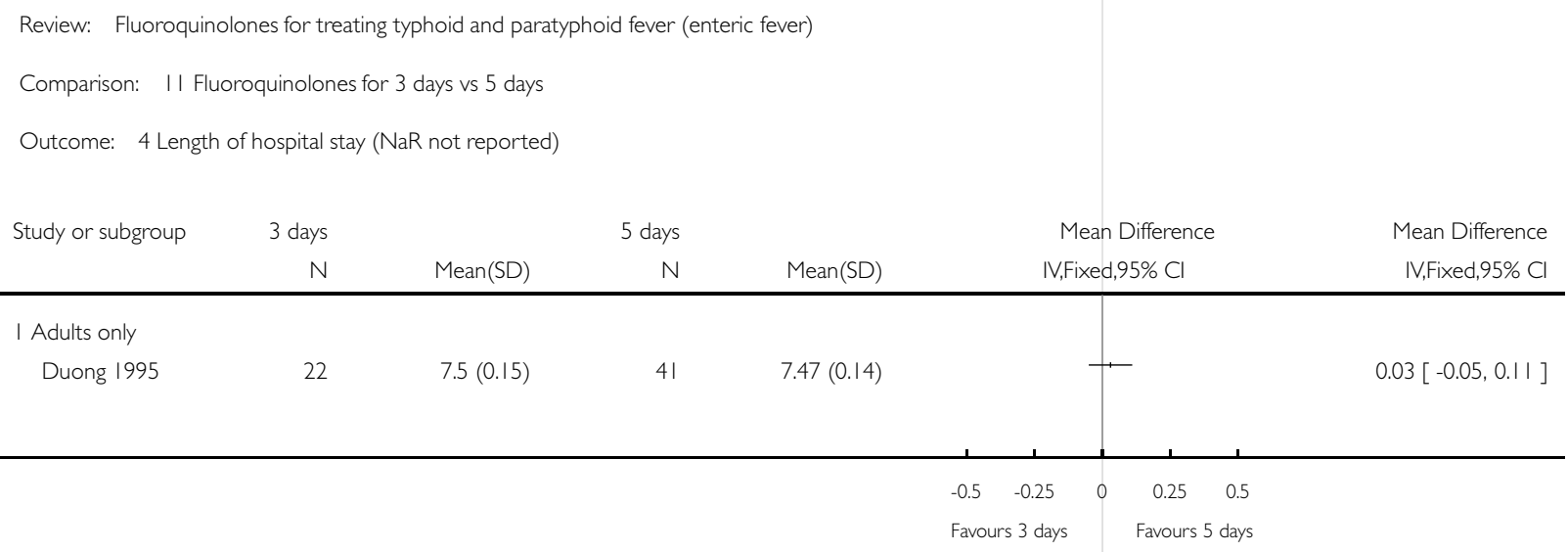


Analysis II.5. Comparison I I Fluoroquinolones for 3 days vs 5 days, Outcome 5 Adverse events (not serious) ( $\mathrm{NaR}$ present).

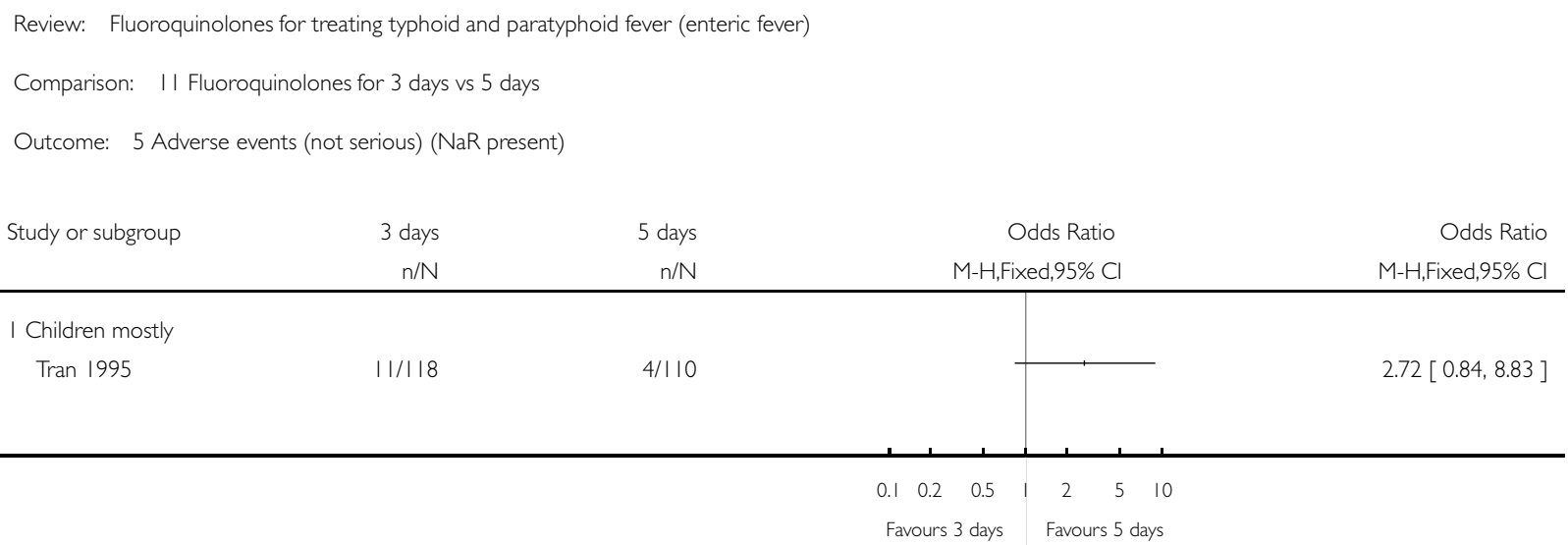

Analysis 12.I. Comparison 12 Fluoroquinolones for 5 days vs 7 days, Outcome I Microbiological failure (NaR not reported).

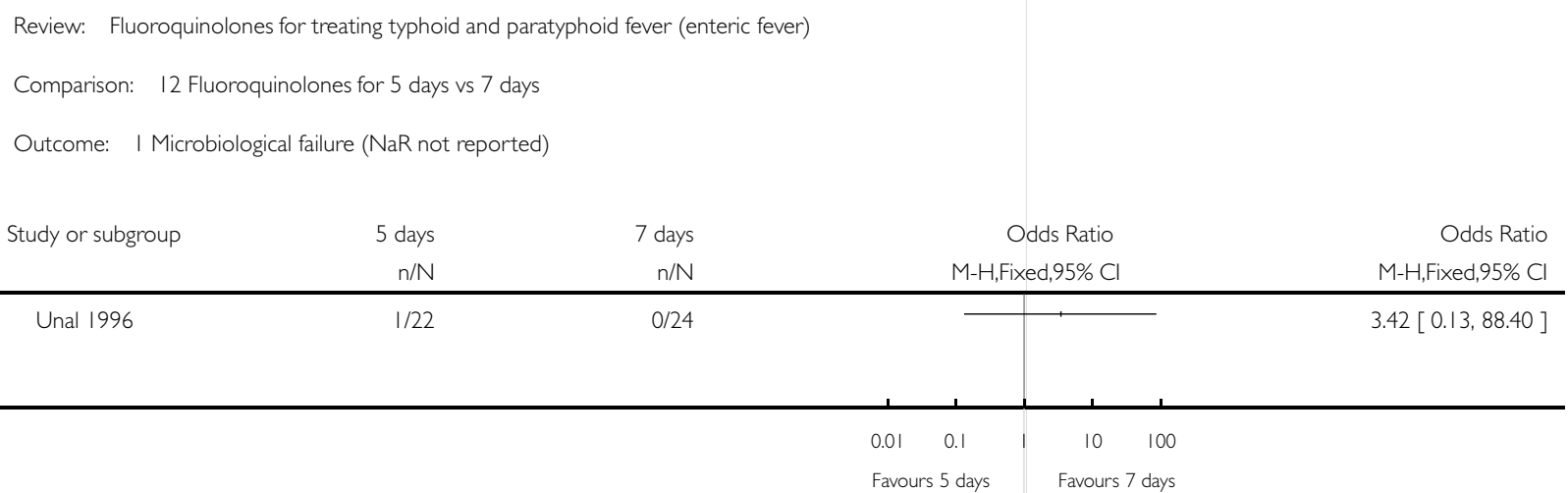


Analysis 12.2. Comparison I 2 Fluoroquinolones for 5 days vs 7 days, Outcome 2 Relapse (NaR not reported).

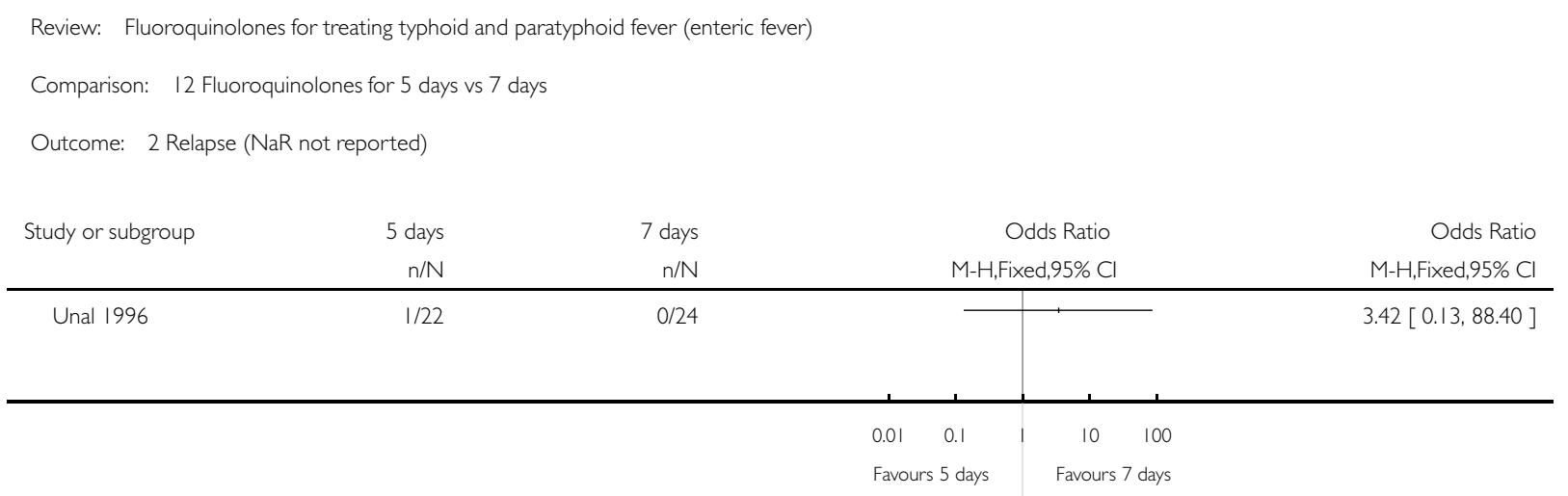

Analysis I 2.3. Comparison I 2 Fluoroquinolones for 5 days vs $\mathbf{7}$ days, Outcome 3 Fever clearance time (NaR not reported).

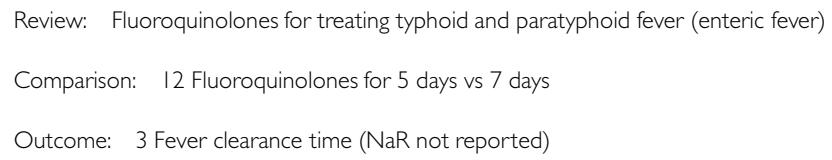

\begin{tabular}{ccccccr} 
& $\mathrm{N}$ & Mean(SD) & $\mathrm{N}$ & Mean(SD) & IV,Fixed,95\% Cl & IV,Fixed,95\% Cl \\
\hline Unal 1996 & 22 & $74.4(24)$ & 24 & $81.6(24)$ & -1 & $-7.20[-21.08,6.68]$
\end{tabular}

$\begin{array}{rrrrr}-100 & -50 & 0 & 50 & 100\end{array}$

Favours 5 days Favours 7 days 


\section{Analysis 12.4. Comparison I2 Fluoroquinolones for 5 days vs 7 days, Outcome 4 Adverse events (not serious) (NaR not reported).}

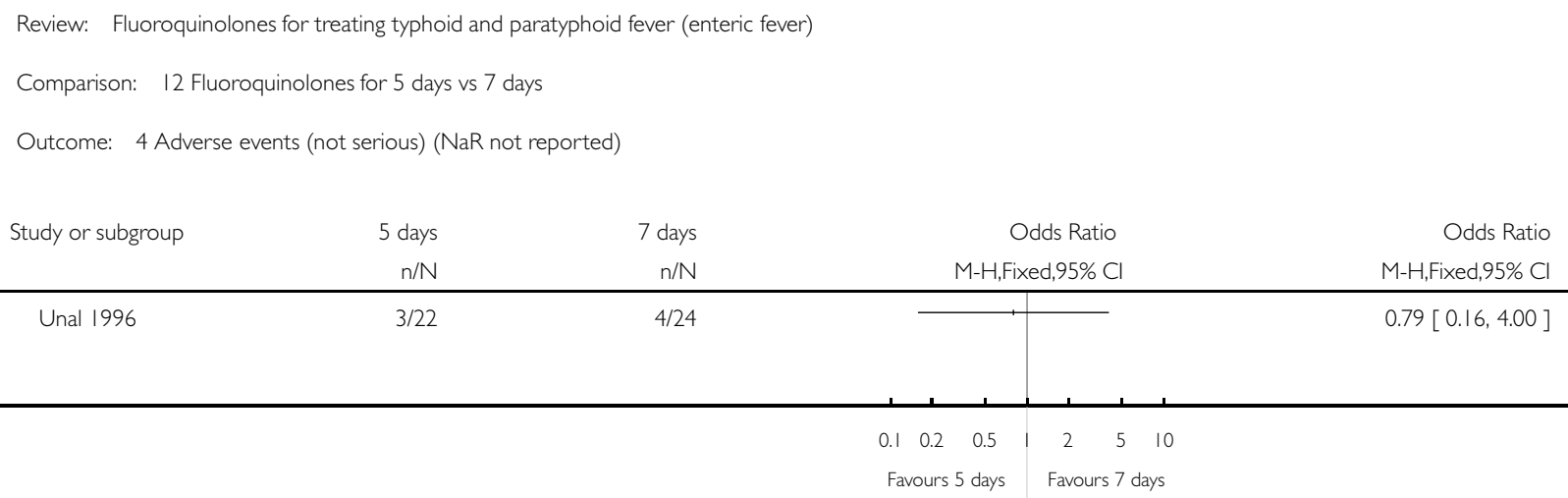

\section{Analysis 13.I. Comparison I3 Fluoroquinolones for 7 days vs 10 or I4 days, Outcome I Microbiological}

failure ( $\mathrm{NaR}$ not reported).

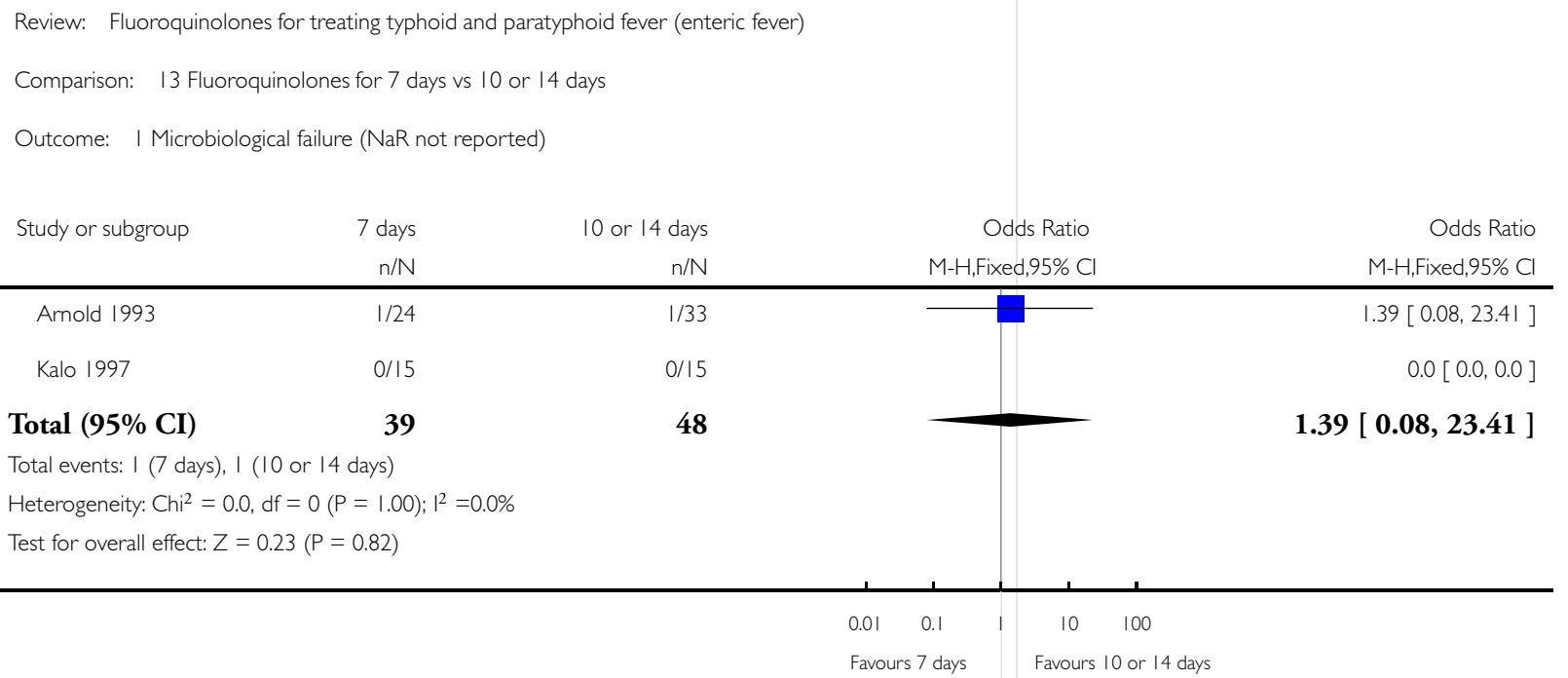


Analysis 13.2. Comparison I 3 Fluoroquinolones for 7 days vs 10 or 14 days, Outcome 2 Relapse (NaR not reported).

\begin{tabular}{|c|c|c|c|c|}
\hline \multicolumn{5}{|c|}{ Comparison: 13 Fluoroquinolones for 7 days vs 10 or 14 days } \\
\hline \multicolumn{5}{|c|}{ Outcome: 2 Relapse (NaR not reported) } \\
\hline \multirow[t]{2}{*}{ Study or subgroup } & 7 days & 10 or 14 days & Odds Ratio & Odds Ratio \\
\hline & $\mathrm{n} / \mathrm{N}$ & $\mathrm{n} / \mathrm{N}$ & M-H,Fixed,95\% Cl & M-H,Fixed,95\% Cl \\
\hline Arnold 1993 & $1 / 24$ & 0/33 & +- & $4.28[0.17,109.61]$ \\
\hline Kalo 1997 & $0 / 15$ & $0 / 15$ & & $0.0[0.0,0.0]$ \\
\hline Total $(95 \% \mathrm{CI})$ & 39 & 48 & - & $4.28[0.17,109.61]$ \\
\hline \multicolumn{5}{|c|}{ Total events: I (7 days), 0 ( 10 or 14 days) } \\
\hline \multicolumn{5}{|c|}{ Heterogeneity: $\mathrm{Chi}^{2}=0.0, \mathrm{df}=0(\mathrm{P}=1.00) ; 1^{2}=0.0 \%$} \\
\hline \multicolumn{5}{|c|}{ Test for overall effect: $Z=0.88(P=0.38)$} \\
\hline
\end{tabular}

Analysis I4.I. Comparison I4 Fluoroquinolones for I0 days vs I4 days, Outcome I Relapse (NaR present).

Review: Fluoroquinolones for treating typhoid and paratyphoid fever (enteric fever)

Comparison: 14 Fluoroquinolones for 10 days vs 14 days

Outcome: I Relapse (NaR present)

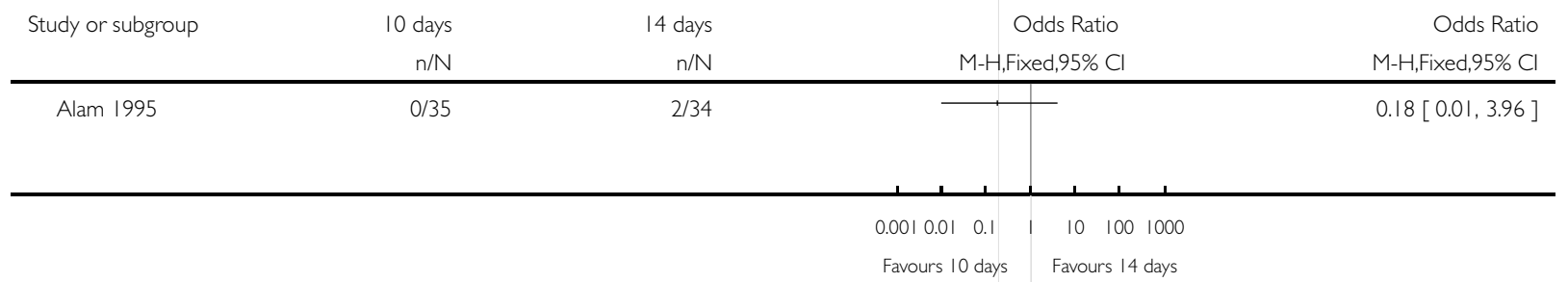


Analysis 14.2. Comparison I4 Fluoroquinolones for 10 days vs 14 days, Outcome 2 Fever clearance time (NaR present).

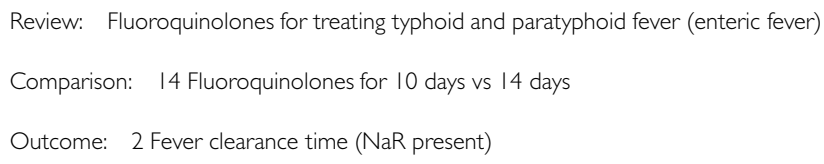

\begin{tabular}{|c|c|c|c|c|c|c|}
\hline \multirow[t]{2}{*}{ Study or subgroup } & \multirow{2}{*}{$\begin{array}{r}10 \text { days } \\
\mathrm{N}\end{array}$} & \multicolumn{3}{|c|}{14 days } & \multirow{2}{*}{$\begin{array}{l}\text { Mean Difference } \\
\text { IV,Fixed,95\% Cl }\end{array}$} & \multirow{2}{*}{$\begin{array}{r}\text { Mean Difference } \\
\text { |V,Fixed,95\% Cl }\end{array}$} \\
\hline & & Mean(SD) & $N$ & Mean(SD) & & \\
\hline Alam 1995 & 35 & $100.8(45.6)$ & 34 & $117.6(62.4)$ & $\longrightarrow$ & $.80[-42.65,9.05$ \\
\hline
\end{tabular}

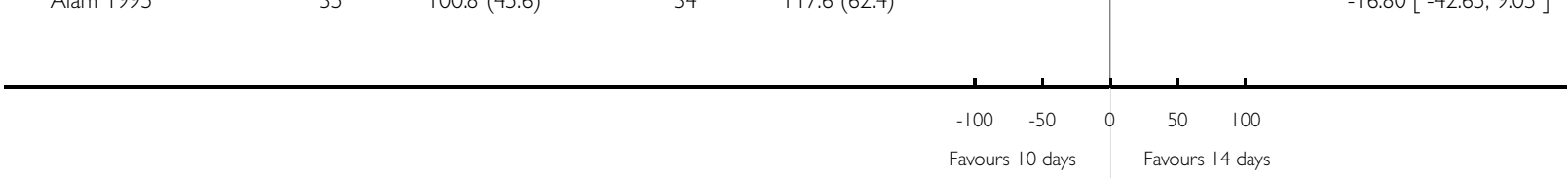

\section{Analysis 14.3. Comparison I4 Fluoroquinolones for I0 days vs I4 days, Outcome 3 Adverse events (not} serious) (NaR present).

Review: Fluoroquinolones for treating typhoid and paratyphoid fever (enteric fever)

Comparison: 14 Fluoroquinolones for 10 days vs 14 days

Outcome: 3 Adverse events (not serious) (NaR present)

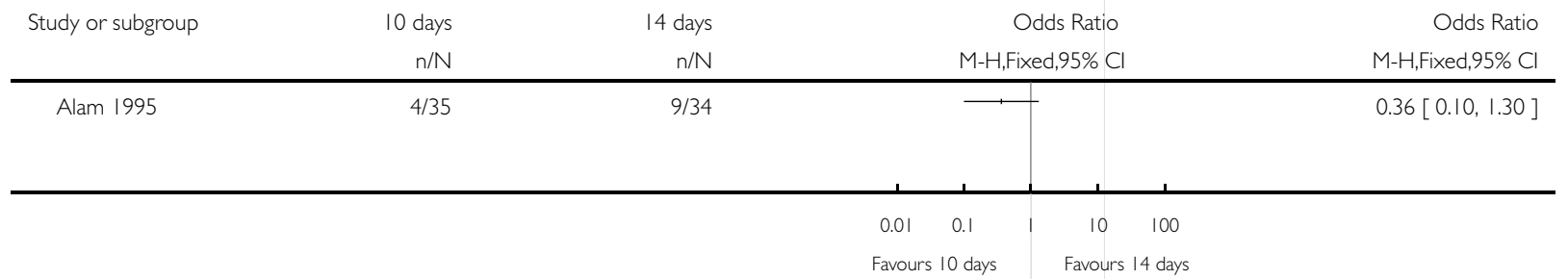

\section{WHAT'S NEW}

Last assessed as up-to-date: 1 April 2008. 
New trials: Five new trials added for the following comparisons: fluoroquinolone vs chloramphenicol (adults, 1 trial); fluoroquinolone vs cefixime (mostly adults, 1 trial); fluoroquinolone vs azithromycin (mostly children, 2 trials); and fluoroquinolone 2 days vs 3 days (children, 1 trial). Two ongoing trials identified and referenced. Two trials were screened for eligibility and excluded (Kumar 2007; Suhendro 2007).

Tables and figures: Updated Table 1 (Microbiology) to include "Multidrug resistance (MDR) defined as", and added minimum inhibitory concentrations (MICs) of fluoroquinolones where nalidixic acid resistance was not stated. (See 'Background' for explanation). Added funnel plots for the fluoroquinolones vs chloramphenicol comparison: Analysis 1.1 (clinical failure), Analysis 1.2 (microbiological failure), and Analysis 1.3 (relapse).

Methods: Durrane Thaver and Asma Azmatullah selected the studies from the updated search results, and extracted data and assessed the methodological quality for the new included trials.

Minor corrections: Analysis 6.6 (Complications: NaR not reported) and Table 2 (Complications): for Wallace 1993 for ceftriaxone group, changed from 1 to 0 (this was described as a 'persistent' complication, and appears to not have developed during treatment).

Analysis 7.4 (Mean fever clearance time: MDR and NaR not reported): for Nalin 1987 (norfloxacin vs chloramphenicol) changed 167.5 hours to corrected value of 160.8 hours for norfloxacin group.

Analysis 11.2 (Relapse), Analysis 11.3 (Fever clearance time), and Analysis 11.5 (Adverse events: not serious): for Tran 1995 changed from children only to children mostly, as stated in text of review.

Table 3 (Definitions of outcomes): For Abejar 1993, moved statement ("blood culture positive at Day 23?") from under "relapse" to "microbiological failure" (resolved disagreement after consensus).

Table 1 (Microbiology): Revision of MDR status for Xiao 1991 and Huai 2000, and included additional information for resistance data for Yu 1998.

Table 2 (Complications): for Gottuzzo 1992, moved "gastrointestinal bleed, others not stated" to correct column.

2 April 2008 New citation required but conclusions have not changed New author: Asma Azmatullah joined the author team. Main changes to the results because of the new trials: For adults, fluoroquinolones had statistically significantly lower fever clearance times compared with chloramphenicol, and also had statistically significantly lower clinical failure and relapse rates compared with cefixime.

Conclusions: Our conclusions are unchanged, that is, 
"data are limited, particularly for children".

Table 1. Microbiology

\begin{tabular}{|c|c|c|c|c|c|c|c|c|}
\hline $\begin{array}{l}\text { Compari- } \\
\text { son }\end{array}$ & Trial & $\begin{array}{l}\text { Partici- } \\
\text { pants }\end{array}$ & $\begin{array}{l}\text { Culture } \\
\text { positive } \\
\text { (site) }\end{array}$ & $\begin{array}{l}\text { S. Typhil } \\
\text { Paratyphi }\end{array}$ & $\begin{array}{l}\text { Number } \\
(\%)^{a} \text { with } \\
\text { MDR }\end{array}$ & $\begin{array}{l}\text { MDR } \\
\text { defined } \text { as }^{b}\end{array}$ & $\begin{array}{l}\text { Number } \\
(\%)^{a} \mathrm{NaR}^{c}\end{array}$ & $\begin{array}{l}\text { Notes on re- } \\
\text { sistance }\end{array}$ \\
\hline \multirow[t]{5}{*}{$\begin{array}{l}\text { Fluoro- } \\
\text { quinolone vs } \\
\text { chloram- } \\
\text { phenicol }\end{array}$} & Abejar 1993 & Not stated & 30 (blood) & $\begin{array}{l}30 / 0 \\
\text { Fluoro- } \\
\text { quinolone: } \\
15 \\
\text { Chloram- } \\
\text { phenicol: } 15\end{array}$ & 0 & $\begin{array}{l}\text { Not stated } \\
\text { No } \\
\text { resistance to } \\
\text { chloram- } \\
\text { pheni- } \\
\text { col in chlo- } \\
\text { rampheni- } \\
\text { col group }\end{array}$ & Not stated & - \\
\hline & $\begin{array}{l}\text { Arnold } \\
1993\end{array}$ & $\begin{array}{l}184 \\
\text { enrolled and } \\
\text { randomized }\end{array}$ & 91 (blood) & $\begin{array}{l}85 / 6 \\
\text { Fluoro- } \\
\text { quinolone } \\
\text { 7-day: } 23 / 1 \\
\text { Fluoro- } \\
\text { quinolone } \\
14 \text {-day: } \\
30 / 3 \\
\text { Chloram- } \\
\text { phenicol: } \\
32 / 2\end{array}$ & Not stated & Not stated & Not stated & - \\
\hline & Bran 1991 & $\begin{array}{l}102 \\
\text { randomized }\end{array}$ & $\begin{array}{l}102 \text { (blood } \\
\text { and/or bone } \\
\text { marrow) }\end{array}$ & $102 / 0$ & 0 & $\begin{array}{l}\text { Not stated } \\
\text { No } \\
\text { resistance to } \\
\text { chloram- } \\
\text { phenicol in } \\
\text { either group }\end{array}$ & Not stated & - \\
\hline & $\begin{array}{l}\text { Cristiano } \\
1995\end{array}$ & $\begin{array}{l}60 \\
\text { enrolled and } \\
\text { randomized }\end{array}$ & 60 (blood) & $\begin{array}{l}60 / 0 \\
\text { Fluoro- } \\
\text { quinolone: } \\
30 \\
\text { Chloram- } \\
\text { phenicol: } 30\end{array}$ & 0 & $\begin{array}{l}\text { Not stated } \\
\text { No } \\
\text { resistance to } \\
\text { chloram- } \\
\text { phenicol, } \\
\text { ampi- } \\
\text { cillin, or co- } \\
\text { trimoxazole }\end{array}$ & $\begin{array}{l}\text { Not stated } \\
\text { MIC range } \\
\text { of pefloxacin } \\
\text { was }<0.016 \\
\text { to } 0.5\end{array}$ & - \\
\hline & Gasem 2003 & $\begin{array}{l}100 \\
\text { enrolled and } \\
\text { randomized }\end{array}$ & $\begin{array}{l}55 \text { (blood } \\
\text { and/or bone } \\
\text { marrow) }\end{array}$ & $50 / 5$ & 0 & $\begin{array}{l}\text { Not stated } \\
\text { No } \\
\text { resistance to }\end{array}$ & $\begin{array}{l}\text { Not stated } \\
\text { MIC range } \\
\text { of ciproflo- }\end{array}$ & - \\
\hline
\end{tabular}


Table 1. Microbiology (Continued)

\begin{tabular}{|c|c|c|c|c|c|c|c|}
\hline & & & & & $\begin{array}{l}\text { chloram- } \\
\text { phenicol } \\
12.8 \% \text { resis- } \\
\text { tant } \\
\text { to ampicillin } \\
\text { or co- } \\
\text { trimoxazole }\end{array}$ & xacin was $<1$ & \\
\hline $\begin{array}{l}\text { Gottuzzo } \\
1992\end{array}$ & Not stated & $\begin{array}{l}98 \quad \text { (not } \\
\text { stated) }\end{array}$ & Not stated & Not stated & Not stated & Not stated & - \\
\hline $\begin{array}{l}\text { Morelli } \\
1992\end{array}$ & $\begin{array}{l}156 \\
\text { enrolled and } \\
\text { randomized }\end{array}$ & 156 (blood) & $156 / 0$ & 0 & $\begin{array}{l}\text { Not stated } \\
\text { MIC range } \\
\text { for chloram- } \\
\text { phenicol } \\
\text { was } 0.5 \text { to } 4 \\
\mathrm{mg} / \mathrm{L}\end{array}$ & $\begin{array}{l}\text { Not stated } \\
\text { MIC } \\
\text { ranges were: } \\
\text { ofloxacin } \\
0.03 \text { to } 0.25 \text {; } \\
\text { pefloxacin } \\
0.06 \text { to } 0.5 \text {; } \\
\text { ciprofloxa- } \\
\text { cin } 0.016 \text { to } \\
0.063 \text {; } \\
\text { enoxacin } \\
0.25 \text {; } \\
\text { norfloxacin } \\
0.063 \text { to } \\
0.25\end{array}$ & - \\
\hline $\begin{array}{l}\text { Phongmany } \\
2005\end{array}$ & $\begin{array}{l}107 \\
\text { enrolled and } \\
\text { randomized }\end{array}$ & 50 (blood) & $\begin{array}{l}50 / 0 \\
\text { Fluoro- } \\
\text { quinolone: } \\
27 \\
\text { Chloram- } \\
\text { phenicol: } 23\end{array}$ & $\begin{array}{l}3 / 50(6 \%) \\
\text { Fluoro- } \\
\text { quinolone: } \\
1 / 27 \\
\text { Chloram- } \\
\text { phenicol: } \\
2 / 23\end{array}$ & $\begin{array}{l}\text { Resistant to } \\
\text { all } 3 \text { (chlo- } \\
\text { rampheni- } \\
\text { col, ampi- } \\
\text { cillin, co-tri- } \\
\text { moxazole) }\end{array}$ & 0 & $\begin{array}{l}\text { Chloram- } \\
\text { phenicol re- } \\
\text { sistance: } \\
4 / 50 \\
\text { Fluoro- } \\
\text { quinolone: } \\
1 / 27 \\
\text { Chloram- } \\
\text { phenicol: } \\
3 / 23^{d} \\
\text { Ampicillin: } \\
5 / 50 \\
\text { Fluoro- } \\
\text { quinolone: } \\
2 / 27 \\
\text { Chloram- } \\
\text { phenicol: } \\
3 / 23 \\
\text { Co-trimoxa- } \\
\text { zole: } 4 / 50\end{array}$ \\
\hline
\end{tabular}


Table 1. Microbiology (Continued)

Fluoro-

quinolone:

$1 / 27$

Chloram-

phenicol:

$3 / 23$

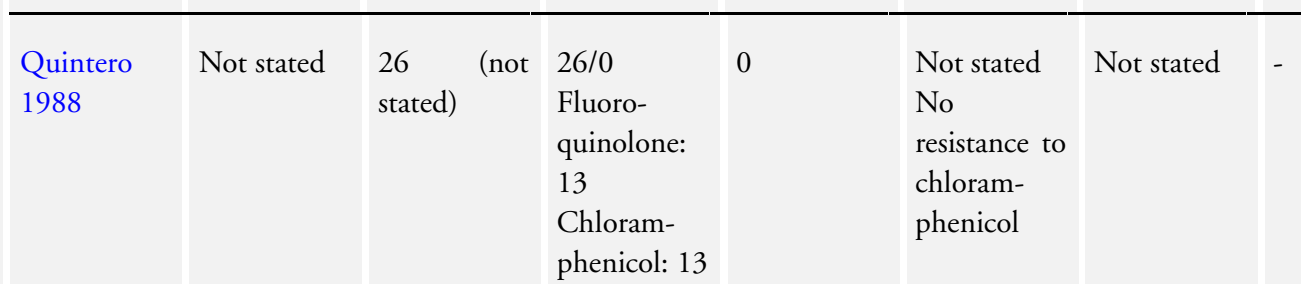

\begin{tabular}{|c|c|c|c|c|c|c|}
\hline Yousaf 1992 & $\begin{array}{l}85 \\
\text { enrolled and } \\
\text { randomized }\end{array}$ & $\begin{array}{l}85 \\
\text { stated) }\end{array}$ & Not stated & Not stated & Not stated & Not stated \\
\hline
\end{tabular}

\begin{tabular}{|c|c|c|c|c|c|c|c|c|}
\hline $\begin{array}{l}\text { Fluoro- } \\
\text { quinolone vs } \\
\text { ampicillin }\end{array}$ & Flores 1994 & Not stated & $\begin{array}{l}40 \quad \text { (not } \\
\text { stated) }\end{array}$ & $\begin{array}{l}40 / 0 \\
\text { Fluoro- } \\
\text { quinolone: } \\
20 \\
\text { Ampicillin: } \\
20\end{array}$ & Not stated & Not stated & Not stated & - \\
\hline \multirow[t]{2}{*}{$\begin{array}{l}\text { Fluoro- } \\
\text { quinolone vs } \\
\text { co- } \\
\text { trimoxazole }\end{array}$} & Hajji 1988 & $\begin{array}{l}77 \\
\text { enrolled and } \\
\text { randomized }\end{array}$ & $\begin{array}{l}42 \text { (blood } \\
\text { and/or } \\
\text { stool) }\end{array}$ & $\begin{array}{l}28 / 4 \\
\text { (from blood } \\
\text { culture) }\end{array}$ & 0 & $\begin{array}{l}\text { Not stated } \\
1 \text { isolate re- } \\
\text { sistant to co- } \\
\text { trimox- } \\
\text { azole was in } \\
\text { pefloxacin } \\
\text { group }\end{array}$ & 0 & - \\
\hline & $\begin{array}{l}\text { Limson } \\
1989\end{array}$ & $\begin{array}{l}53 \\
\text { enrolled and } \\
\text { randomized }\end{array}$ & 40 (blood) & $\begin{array}{l}28 / 12 \\
\text { Fluoro- } \\
\text { quinolone: } \\
15 / 5 \\
\text { Co-trimoxa- } \\
\text { zole: } 13 / 7\end{array}$ & 0 & $\begin{array}{l}\text { Not stated } \\
\text { No resis- } \\
\text { tance to co- } \\
\text { trimoxazole } \\
16 \text { were re- } \\
\text { sistant } \\
\text { to chloram- } \\
\text { phenicol }\end{array}$ & Not stated & - \\
\hline $\begin{array}{l}\text { Fluoro- } \\
\text { quinolone vs } \\
\text { azithromy- } \\
\text { cin }\end{array}$ & $\begin{array}{l}\text { Dolecek } \\
2008\end{array}$ & $\begin{array}{l}358 \\
\text { enrolled and } \\
\text { randomized }\end{array}$ & $\begin{array}{l}288 \text { (blood } \\
\text { or bone } \\
\text { marrow) }\end{array}$ & $\begin{array}{l}282 / 5 \\
\text { Fluoro- } \\
\text { quinolone: } \\
144 / 1 \\
\text { Azithromy- } \\
\text { cin: } 138 / 4\end{array}$ & $\begin{array}{l}153 \quad(58 \%) \\
\text { of } 263 S . \text { Ty- } \\
\text { phi } \\
\text { Fluoro- } \\
\text { quinolone: } \\
87 / 137 \\
\text { Azithromy- }\end{array}$ & $\begin{array}{l}\text { Resistant to } \\
\text { all } 3 \text { (chlo- } \\
\text { rampheni- } \\
\text { col, ampi- } \\
\text { cillin, co-tri- } \\
\text { moxazole) }\end{array}$ & $\begin{array}{l}253 \quad(96 \%) \\
\text { of } 263 S . \text { Ty- } \\
\text { phi } \\
\text { Fluoro- } \\
\text { quinolone: } \\
132 / 137 \\
\text { Azithromy- }\end{array}$ & $\begin{array}{l}\text { All } 5 \\
S . \text { Paratyphi } \\
\text { were suscep- } \\
\text { tible }\end{array}$ \\
\hline
\end{tabular}

Fluoroquinolones for treating typhoid and paratyphoid fever (enteric fever) (Review)

Copyright ( 2008 The Cochrane Collaboration. Published by John Wiley \& Sons, Ltd. 
Table 1. Microbiology (Continued)

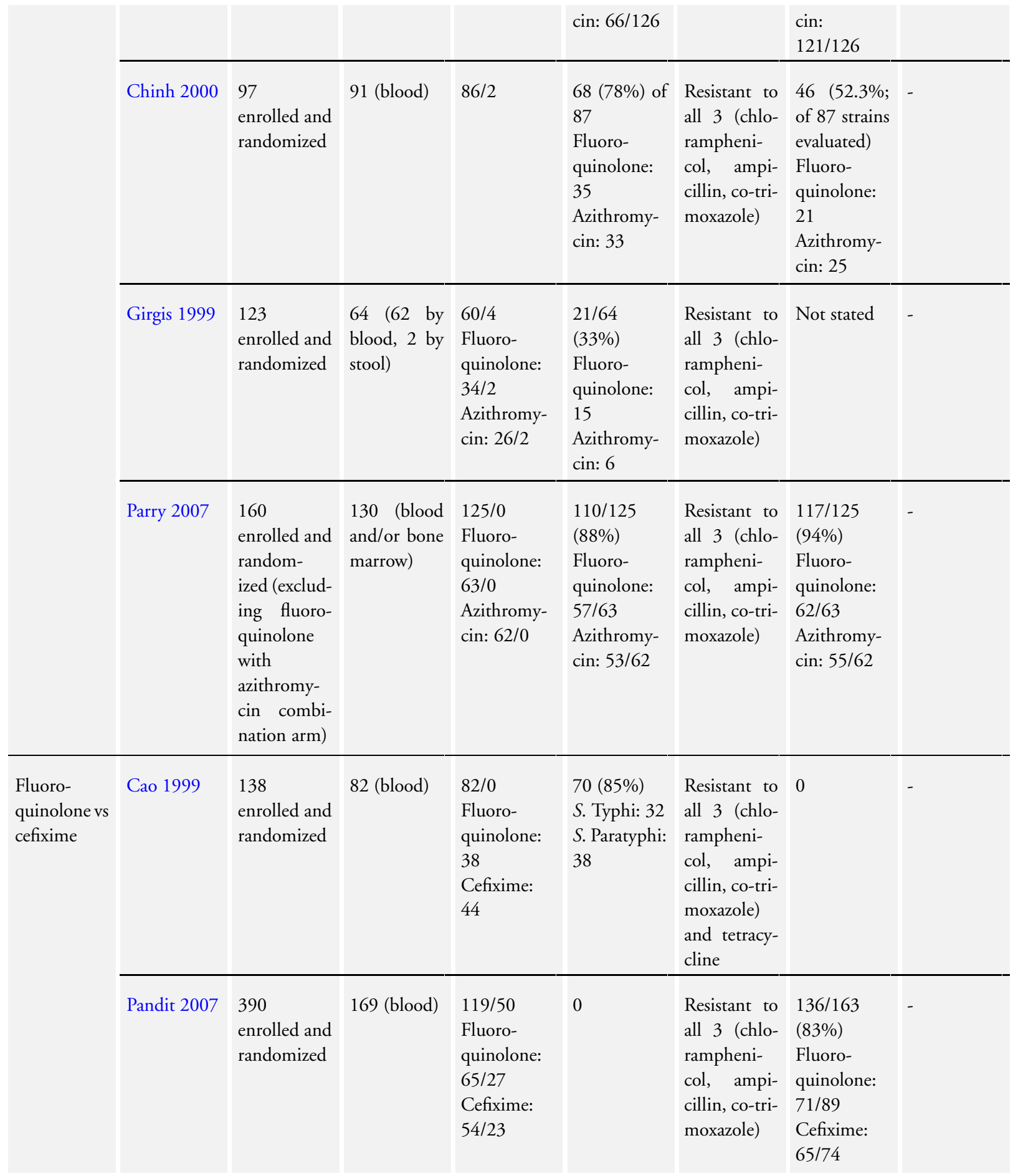


Table 1. Microbiology (Continued)

\begin{tabular}{|c|c|c|c|c|c|c|c|c|}
\hline & Yu 1998 & $\begin{array}{l}80 \text { random- } \\
\text { ized }\end{array}$ & $\begin{array}{l}80 \text { (blood or } \\
\text { bone } \\
\text { marrow) }\end{array}$ & $\begin{array}{l}40 / 40 \\
\text { Fluoro- } \\
\text { quinolone: } \\
21 / 19 \\
\text { Cefixime: } \\
19 / 21\end{array}$ & $\begin{array}{l}\text { Not stated } \\
\text { Individual } \\
\text { drug resis- } \\
\text { tance was re- } \\
\text { ported, but } \\
\text { reported to- } \\
\text { gether with } \\
\text { isolates in- } \\
\text { volved in an- } \\
\text { other trial }\end{array}$ & Not stated & $\begin{array}{l}\text { Not stated } \\
\text { MIC for lev- } \\
\text { ofloxacin } \\
\text { was } \leq 0.03 \\
\text { to } 1 \mathrm{mg} / \mathrm{L} \text {, } \\
\text { but reported } \\
\text { together } \\
\text { with isolates } \\
\text { involved in } \\
\text { another trial }\end{array}$ & $\begin{array}{l}3 / 98 \text { strains } \\
\text { resistant } \\
\text { to cefixime, } \\
\text { but unclear } \\
\text { which arm } \\
\text { these were in } \\
\text { and } \\
\text { also were not } \\
\text { separated } \\
\text { from isolates } \\
\text { involved in } \\
\text { another trial }\end{array}$ \\
\hline \multirow[t]{3}{*}{$\begin{array}{l}\text { Fluoro- } \\
\text { quinolone vs } \\
\text { ceftriaxone }\end{array}$} & Smith 1994 & $\begin{array}{l}60 \\
\text { enrolled and } \\
\text { randomized }\end{array}$ & $\begin{array}{l}47 \\
\text { ( } 44 \text { by blood } \\
\text { and/or bone } \\
\text { marrow, } 3 \\
\text { by stool) }\end{array}$ & $\begin{array}{l}41 / 6 \\
\text { Fluoro- } \\
\text { quinolone: } \\
21 / 1 \\
\text { Ceftriaxone: } \\
20 / 5\end{array}$ & $\begin{array}{l}26(55 \%) \\
\text { Fluoro- } \\
\text { quinolone: } \\
14 \\
\text { Ceftriaxone: } \\
12\end{array}$ & $\begin{array}{l}\text { Resistant to } \\
\text { all } 3 \text { (chlo- } \\
\text { rampheni- } \\
\text { col, ampi- } \\
\text { cillin, co-tri- } \\
\text { moxazole) } \\
\text { and tetracy- } \\
\text { cline }\end{array}$ & 0 & - \\
\hline & Tran 1994 & $\begin{array}{l}46 \\
\text { enrolled and } \\
\text { randomized }\end{array}$ & 31 (blood) & $\begin{array}{l}27 / 4 \\
\text { Fluoro- } \\
\text { quinolone: } \\
14 / 2 \\
\text { Ceftriaxone: } \\
13 / 2\end{array}$ & $12(38 \%)$ & $\begin{array}{l}\text { Resistant to } \\
\text { all } 3 \text { (chlo- } \\
\text { rampheni- } \\
\text { col, ampi- } \\
\text { cillin, co-tri- } \\
\text { moxazole) }\end{array}$ & $\begin{array}{l}\text { Not stated } \\
\text { MIC for } \\
\text { fleroxacin } \\
\text { was mostly } \\
0.06 \text { (addi- } \\
\text { tional infor- } \\
\text { mation from } \\
\text { trial author) }\end{array}$ & - \\
\hline & $\begin{array}{l}\text { Wallace } \\
1993\end{array}$ & $\begin{array}{l}43 \text { enrolled } \\
\text { and } 42 \text { ran- } \\
\text { domized }\end{array}$ & 42 (blood) & $\begin{array}{l}42 / 0 \\
\text { Fluoro- } \\
\text { quinolone: } \\
20 \\
\text { Ceftriaxone: } \\
22\end{array}$ & $\begin{array}{l}22(52 \%) \\
\text { Fluoro- } \\
\text { quinolone: } \\
11 \\
\text { Ceftriaxone: } \\
11\end{array}$ & $\begin{array}{l}\text { Resistant to } \\
\text { all } 3 \text { (chlo- } \\
\text { rampheni- } \\
\text { col, ampi- } \\
\text { cillin, co-tri- } \\
\text { moxazole) }\end{array}$ & Not stated & - \\
\hline $\begin{array}{l}\text { Norfloxacin } \\
\text { vs chloram- } \\
\text { phenicol }\end{array}$ & Nalin 1987 & $\begin{array}{l}184 \\
\text { enrolled and } \\
\text { randomized }\end{array}$ & $\begin{array}{l}169 \text { (not } \\
\text { stated) }\end{array}$ & $\begin{array}{l}169 / 0 \\
\text { Fluoro- } \\
\text { quinolone: } \\
90 \\
\text { Chloram- } \\
\text { phenicol: } 79\end{array}$ & Not stated & $\begin{array}{l}\text { Not stated } \\
1 \text { isolate } \\
\text { with 'inter- } \\
\text { mediate sus- } \\
\text { ceptibil- } \\
\text { ity' in nor- } \\
\text { floxacin } \\
\text { group }\end{array}$ & Not stated & - \\
\hline
\end{tabular}


Table 1. Microbiology (Continued)

\begin{tabular}{|c|c|c|c|c|c|c|c|c|}
\hline & Sarma 1991 & $\begin{array}{l}40 \\
\text { enrolled and } \\
\text { randomized }\end{array}$ & $\begin{array}{l}40 \quad \text { (blood } \\
\text { and/or bone } \\
\text { marrow) }\end{array}$ & $38 / 2$ & $8(20 \%)$ & Not stated & $\begin{array}{l}4(10 \%) \text { (all } \\
\text { reported as } \\
\text { resistant to } \\
\text { norfloxacin) }\end{array}$ & - \\
\hline $\begin{array}{l}\text { Norfloxacin } \\
\text { vs } \\
\text { ceftriaxone }\end{array}$ & Huai 2000 & $\begin{array}{l}196 \\
\text { enrolled, but } \\
\text { only } 60 \text { cases } \\
\text { resistant to } \\
\text { ampi- } \\
\text { cillin, co-tri- } \\
\text { moxazole, or } \\
\text { chloram- } \\
\text { pheni- } \\
\text { col were ran- } \\
\text { domized }\end{array}$ & $\begin{array}{l}60 \quad(56 \text { by } \\
\text { blood, } 4 \text { by } \\
\text { bone } \\
\text { marrow) }\end{array}$ & $\begin{array}{l}60 / 0 \\
\text { Fluoro- } \\
\text { quinolone: } \\
30 \\
\text { Ceftriaxone: } \\
30\end{array}$ & Not stated & $\begin{array}{l}\text { Not stated } \\
\text { All } \\
\text { described as } \\
\text { resistant to } \\
\text { ampi- } \\
\text { cillin, chlo- } \\
\text { rampheni- } \\
\text { col, or co- } \\
\text { trimoxazole }\end{array}$ & Not stated & - \\
\hline \multirow[t]{4}{*}{$\begin{array}{l}\text { Fluoro- } \\
\text { quinolone vs } \\
\text { an- } \\
\text { other fluoro- } \\
\text { quinolone }\end{array}$} & Bai 1995 & $\begin{array}{l}102 \\
\text { randomized }\end{array}$ & $\begin{array}{l}102 \text { (blood } \\
\text { or bone } \\
\text { marrow) }\end{array}$ & $\begin{array}{l}102 / 0 \\
\text { Nor- } \\
\text { floxacin: } 50 \\
\text { Enoxacin: } \\
52\end{array}$ & Not stated & Not stated & Not stated & - \\
\hline & Jia 1994 & $\begin{array}{l}130 \\
\text { randomized }\end{array}$ & $\begin{array}{l}130 \text { (blood } \\
\text { or bone } \\
\text { marrow) }\end{array}$ & $\begin{array}{l}130 / 0 \\
\text { Nor- } \\
\text { floxacin: } 67 \\
\text { Pefloxacin: } \\
63\end{array}$ & Not stated & Not stated & Not stated & - \\
\hline & Xiao 1991 & $\begin{array}{l}40 \text { random- } \\
\text { ized } \\
\text { groups })\end{array}$ & $\begin{array}{l}37 \text { (blood or } \\
\text { bone } \\
\text { marrow) (6 } \\
\text { groups) }\end{array}$ & $37 / 0$ & Not stated & $\begin{array}{l}\text { Not stated. } \\
\text { All resistant } \\
\text { to chloram- } \\
\text { phenicol; } 1 \\
\text { resistant to } \\
\text { norfloxacin; } \\
4 \text { resistant to } \\
\text { pefloxacin }\end{array}$ & $\begin{array}{l}\text { Not stated } \\
\text { For } 30 \text { iso- } \\
\text { lates tested, } \\
\text { MIC range } \\
\text { for ciproflo- } \\
\text { xacin was } \\
0.03 \text { to } 1 \text {; } \\
\text { norfloxacin } \\
<0.03 \text { to } \\
2 \text {; ofloxacin } \\
0.03 \text { to } \\
8 \text {; pefloxacin } \\
0.03 \text { to } 2\end{array}$ & - \\
\hline & Yang 1991 & $\begin{array}{l}56 \text { random- } \\
\text { ized }\end{array}$ & 56 (blood) & $\begin{array}{l}56 / 0 \\
\text { Nor- } \\
\text { floxacin: } 28 \\
\text { Ofloxacin: } \\
28\end{array}$ & Not stated & $\begin{array}{l}\text { Not stated } \\
\text { Resistance } \\
\text { to chloram- } \\
\text { pheni- } \\
\text { col in } 18 / 27 \text {, } \\
\text { ampicillin in }\end{array}$ & $\begin{array}{l}\text { Not stated } \\
\text { Resis- } \\
\text { tance to nor- } \\
\text { floxacin: } 3 \\
\text { (in ofloxacin } \\
\text { group) }\end{array}$ & - \\
\hline
\end{tabular}


Table 1. Microbiology (Continued)

\begin{tabular}{|c|c|c|c|c|c|c|c|c|}
\hline & & & & & & $\begin{array}{l}24 / 28 \text {, and } \\
\text { co- } \\
\text { trimoxazole } \\
\text { in } 25 / 26 \text { iso- } \\
\text { lates } \\
\text { tested from } \\
\text { ofloxacin } \\
\text { group }\end{array}$ & & \\
\hline \multirow[t]{5}{*}{$\begin{array}{l}\text { Dif- } \\
\text { ferent dura- } \\
\text { tions of fluo- } \\
\text { roquinolone }\end{array}$} & Alam 1995 & $\begin{array}{l}76 \\
\text { enrolled and } \\
\text { randomized }\end{array}$ & $\begin{array}{l}72 \text { (blood or } \\
\text { bone } \\
\text { marrow) }\end{array}$ & $\begin{array}{l}61 / 8 \\
\text { Fluoro- } \\
\text { quinolone } \\
10-\text { day: } \\
30 / 5 \\
\text { Fluoro- } \\
\text { quinolone } \\
14 \text {-day: } \\
31 / 3\end{array}$ & $\begin{array}{l}36 / 69 \\
(52 \%) \\
\text { Fluoro- } \\
\text { quinolone } \\
\text { 10-day: } 18 \\
\text { Fluoro- } \\
\text { quinolone } \\
\text { 14-day: } 18\end{array}$ & $\begin{array}{l}\text { Resistance } \\
\text { to all drugs } \\
\text { used } \\
\text { convention- } \\
\text { ally against } \\
S \text {. Typhi and } \\
S \text {. Paratyphi }\end{array}$ & $\begin{array}{l}5 / 69(7 \%) \\
\text { Fluoro- } \\
\text { quinolone } \\
\text { 10-day: } 2 \\
\text { Fluoro- } \\
\text { quinolone } \\
\text { 14-day: } 3 \\
\text { (derived } \\
\text { from data } \\
\text { presented } \\
\text { for MIC for } \\
\text { ciprofloxa- } \\
\text { cin) }\end{array}$ & - \\
\hline & $\begin{array}{l}\text { Duong } \\
1995\end{array}$ & $\begin{array}{l}95 \\
\text { enrolled and } \\
\text { randomized }\end{array}$ & $\begin{array}{l}63 \text { (blood or } \\
\text { bone } \\
\text { marrow) }\end{array}$ & $\begin{array}{l}62 / 1 \\
\text { Fluoro- } \\
\text { quinolone } \\
5 \text {-day: 40/1 } \\
\text { Fluoro- } \\
\text { quinolone } \\
\text { 3-day: 22/0 }\end{array}$ & $\begin{array}{l}\text { Around } \\
80 \% \\
\text { [trialist's es- } \\
\text { timate] }\end{array}$ & $\begin{array}{l}\text { Not } \\
\text { available }\end{array}$ & Not stated & - \\
\hline & Kalo 1997 & $\begin{array}{l}30 \\
\text { ampicillin- } \\
\text { resistant) } \\
\text { enrolled and } \\
\text { randomized }\end{array}$ & 30 (blood) & $30 / 0$ & $\begin{array}{l}12 / 30 \\
(40 \%)\end{array}$ & $\begin{array}{l}\text { Resistant to } \\
\text { all } 3 \text { (chlo- } \\
\text { rampheni- } \\
\text { col, ampi- } \\
\text { cillin, co-tri- } \\
\text { moxazole) }\end{array}$ & Not stated & - \\
\hline & $\begin{array}{l}\text { Nguyen } \\
1997\end{array}$ & $\begin{array}{l}107 \\
\text { enrolled and } \\
\text { randomized }\end{array}$ & 101 (blood) & $\begin{array}{l}95 / 5 \\
\text { Fluoro- } \\
\text { quinolone } \\
\text { 2-day: 43/4 } \\
\text { Fluoro- } \\
\text { quinolone } \\
\text { 3-day: 52/1 }\end{array}$ & $\begin{array}{l}75 / 95 \\
(79 \%) \\
\text { Fluoro- } \\
\text { quinolone } \\
\text { 2-day: } 35 \\
\text { Fluoro- } \\
\text { quinolone } \\
\text { 3-day: } 40\end{array}$ & $\begin{array}{l}\text { Resistant to } \\
\text { all } 3 \text { (chlo- } \\
\text { rampheni- } \\
\text { col, ampi- } \\
\text { cillin, co-tri- } \\
\text { moxazole) } \\
\text { and tetracy- } \\
\text { cline }\end{array}$ & $\begin{array}{l}\text { 5/95 (5\%) } \\
\text { Fluoro- } \\
\text { quinolone } \\
\text { 2-day: } 1 \\
\text { Fluoro- } \\
\text { quinolone } \\
\text { 3-day: } 4\end{array}$ & - \\
\hline & Tran 1995 & $\begin{array}{ll}438 & \text { en- } \\
\text { rolled, } & 425\end{array}$ & 228 (blood) & $\begin{array}{l}207 / 19 \\
(2 \quad \text { other }\end{array}$ & $\begin{array}{l}189 \\
\text { Fluoro- }\end{array}$ & $\begin{array}{l}\text { Resistant to } \\
\text { standard an- }\end{array}$ & $\begin{array}{l}\text { Few } \\
\mathrm{NaR} \text { strains }\end{array}$ & - \\
\hline
\end{tabular}


Table 1. Microbiology (Continued)

\begin{tabular}{|c|c|c|c|c|c|c|c|}
\hline & randomized & & Salmonella) & $\begin{array}{l}\text { quinolone } \\
\text { 3-day: } 98 \\
\text { Fluoro- } \\
\text { quinolone } \\
\text { 5-day: } 91\end{array}$ & tibiotics & $\begin{array}{l}\text { present, } \\
\text { number not } \\
\text { stated }\end{array}$ & \\
\hline Unal 1996 & $\begin{array}{l}46 \text { random- } \\
\text { ized }\end{array}$ & $\begin{array}{l}46 \text { (blood } \\
\text { and/or bone } \\
\text { marrow) }\end{array}$ & $\begin{array}{l}19 / 27 \\
\text { Fluoro- } \\
\text { quinolone } \\
5 \text {-day: } 8 / 14 \\
\text { Fluoro- } \\
\text { quinolone } \\
7 \text {-day: } \\
11 / 13\end{array}$ & $\begin{array}{l}\text { 6/46 (13\%) } \\
\text { Fluoro- } \\
\text { quinolone } \\
5 \text {-day: } 3 \\
\text { Fluoro- } \\
\text { quinolone } \\
\text { 7-day: } 3\end{array}$ & $\begin{array}{l}\text { Resistant to } \\
\text { all } 3 \text { (chlo- } \\
\text { rampheni- } \\
\text { col, ampi- } \\
\text { cillin, co-tri- } \\
\text { moxazole) }\end{array}$ & $\begin{array}{l}\text { Not stated } \\
\text { MIC for pe- } \\
\text { floxacin was } \\
0.06 \text { to } 1\end{array}$ & - \\
\hline Vinh 1996 & $\begin{array}{l}108 \\
\text { enrolled and } \\
\text { randomized }\end{array}$ & 100 (blood) & $\begin{array}{l}\text { 100/0 } \\
\text { Fluoro- } \\
\text { quinolone } \\
\text { 2-day: } 53 \\
\text { Fluoro- } \\
\text { quinolone } \\
\text { 3-day: } 47\end{array}$ & $\begin{array}{l}84 \\
\text { Fluoro- } \\
\text { quinolone } \\
\text { 2-day: } 46 \\
\text { Fluoro- } \\
\text { quinolone } \\
\text { 3-day: } 38\end{array}$ & $\begin{array}{l}\text { Resistant to } \\
\text { all } 3 \text { (chlo- } \\
\text { rampheni- } \\
\text { col, ampi- } \\
\text { cillin, co-tri- } \\
\text { moxazole) } \\
\text { and tetracy- } \\
\text { cline }\end{array}$ & $\begin{array}{l}13(13 \%) \\
\text { Fluoro- } \\
\text { quinolone } \\
\text { 2-day: } 6 \\
\text { Fluoro- } \\
\text { quinolone } \\
\text { 3-day: } 7\end{array}$ & - \\
\hline Vinh 2005 & $\begin{array}{l}235 \\
\text { enrolled and } \\
\text { randomized }\end{array}$ & 202 (blood) & $\begin{array}{l}\text { 196/0 } \\
\text { Fluoro- } \\
\text { quinolone } \\
\text { 2-day: } 89 \\
\text { Fluoro- } \\
\text { quinolone } \\
\text { 3-day: } 107\end{array}$ & $\begin{array}{l}\text { 176/196 } \\
(90 \%) \\
\text { Fluoro- } \\
\text { quinolone } \\
\text { 2-day: } \\
82 / 89 \\
\text { Fluoro- } \\
\text { quinolone } \\
\text { 3-day: } \\
94 / 107\end{array}$ & $\begin{array}{l}\text { Resistant to } \\
\text { all } 3 \text { (chlo- } \\
\text { rampheni- } \\
\text { col, ampi- } \\
\text { cillin, co-tri- } \\
\text { moxazole) }\end{array}$ & $\begin{array}{l}4 / 161 \\
(2.5 \%) \\
\text { Fluoro- } \\
\text { quinolone } \\
\text { 2-day: 1/72 } \\
\text { Fluoro- } \\
\text { quinolone } \\
\text { 3-day: 3/89 }\end{array}$ & - \\
\hline
\end{tabular}

MDR: multiple-drug-resistant strain; MIC: minimum inhibitory concentration; NaR: nalidixic acid-resistant strain.

${ }^{a}$ Calculation: number with MDR or NaR divided by number culture positive.

${ }^{b}$ As stated or implied in text of report.

${ }^{c}$ Or MIC of fluoroquinolone if available (all ranges in $\mathrm{mg} / \mathrm{L}$ ).

${ }^{d}$ These participants were switched to fluoroquinolone when organisms were found resistant to assigned drug.

Table 2. Complications ${ }^{a}$

\begin{tabular}{l|ll} 
Comparison & Trial & No. of participants (in brackets) with complications ${ }^{b}$ \\
\cline { 3 - 4 } & Intervention & Control
\end{tabular}


Table 2. Complications ${ }^{a} \quad$ (Continued)

\begin{tabular}{|c|c|c|c|}
\hline \multirow[t]{4}{*}{$\begin{array}{l}\text { Fluoroquinolone vs chloram- } \\
\text { phenicol }\end{array}$} & Arnold 1993 & \multicolumn{2}{|c|}{$\begin{array}{l}\text { Gastrointestinal haemorrhage, gastrointestinal perforation, and pneumonia } \\
\text { occurred, but report does not say in which group, and trial combined both } \\
\text { culture-negative and culture-positive participants }\end{array}$} \\
\hline & Gasem 2003 & Ciprofloxacin: pneumonia (3) & $\begin{array}{l}\text { Chloramphenicol: sepsis, myocardi- } \\
\text { tis, pneumonia (1); gastrointestinal } \\
\text { bleed (1); pneumonia (1) }\end{array}$ \\
\hline & Gottuzzo 1992 & $\begin{array}{l}\text { Ciprofloxacin: gastrointestinal bleed } \\
\text { (1); others not described }\end{array}$ & Not described \\
\hline & Phongmany 2005 & Ofloxacin: 0 & $\begin{array}{l}\text { Chloramphenicol: gastrointestinal } \\
\text { bleed and perforation (1) }\end{array}$ \\
\hline $\begin{array}{l}\text { Fluoroquinolone vs co-trimox- } \\
\text { azole }\end{array}$ & Hajji 1988 & 0 & 0 \\
\hline \multirow[t]{4}{*}{$\begin{array}{l}\text { Fluoroquinolone vs azithromy- } \\
\text { cin }\end{array}$} & Dolecek 2008 & Gatifloxacin: 0 & $\begin{array}{l}\text { Azithromycin: liver dysfunction (2); } \\
\text { pneumonia (2); gastrointestinal bleed } \\
\text { (4) }\end{array}$ \\
\hline & Chinh 2000 & Ofloxacin: gastrointestinal bleed (1) & $\begin{array}{l}\text { Azithromycin: gastrointestinal bleed } \\
\text { (1) }\end{array}$ \\
\hline & Girgis 1999 & 0 & 0 \\
\hline & Parry 2007 & Ofloxacin: 0 & $\begin{array}{l}\text { Azithromycin: gastrointestinal bleed } \\
\text { (2) }\end{array}$ \\
\hline \multirow[t]{3}{*}{ Fluoroquinolone vs cefixime } & Cao 1999 & $\begin{array}{l}\text { Ofloxacin: death (1); small gastroin- } \\
\text { testinal bleed (1) }\end{array}$ & $\begin{array}{l}\text { Cefixime: required blood transfusion } \\
\text { (1) }\end{array}$ \\
\hline & Pandit 2007 & Gatifloxacin: 0 & $\begin{array}{l}\text { Cefixime: death with gastrointesti- } \\
\text { nal bleed; thrombocytopenia; dissem- } \\
\text { inated intravascular coagulation (1) }\end{array}$ \\
\hline & Yu 1998 & 0 & 0 \\
\hline \multirow[t]{3}{*}{ Fluoroquinolone vs ceftriaxone } & Smith 1994 & Ofloxacin: 0 & $\begin{array}{l}\text { Ceftriaxone: anaemia (1); jaundice } \\
\text { and anaemia (1) }\end{array}$ \\
\hline & Tran 1994 & 0 & 0 \\
\hline & Wallace 1993 & 0 & 0 \\
\hline \multirow[t]{2}{*}{$\begin{array}{l}\text { Norfloxacin vs chlorampheni- } \\
\text { col }\end{array}$} & Nalin 1987 & $\begin{array}{l}\text { Norfloxacin: gastrointestinal bleed } \\
\text { (number unclear) }\end{array}$ & Chloramphenicol: 0 \\
\hline & Sarma 1991 & 0 & 0 \\
\hline
\end{tabular}


Table 2. Complications ${ }^{a} \quad$ (Continued)

\begin{tabular}{|c|c|c|c|}
\hline \multirow{4}{*}{$\begin{array}{l}\text { Norfloxacin vs other fluoro- } \\
\text { quinolones }\end{array}$} & Bai 1995 & 0 & 0 \\
\hline & Jia 1994 & 0 & 0 \\
\hline & Xiao 1991 & $\begin{array}{l}\text { Gastrointestinal bleeds (2) and en- } \\
\text { cephalopathy (1): unclear which } \\
\text { group they occurred in }\end{array}$ & $\begin{array}{l}\text { Gastrointestinal bleeds (2) and en- } \\
\text { cephalopathy (1): unclear which } \\
\text { group they occurred in }\end{array}$ \\
\hline & Yang 1991 & Not described & Not described \\
\hline \multirow{4}{*}{$\begin{array}{l}\text { Different durations of fluoro- } \\
\text { quinolones }\end{array}$} & Duong 1995 & 0 & 0 \\
\hline & Nguyen 1997 & 2-day: 0 & $\begin{array}{l}\text { 3-day: gastrointestinal bleed (2); jaun- } \\
\text { dice (1); hypotension (1) }\end{array}$ \\
\hline & Vinh 1996 & 2-day: 0 & $\begin{array}{l}\text { 3-day: delirium (1); gastrointestinal } \\
\text { bleed (1) }\end{array}$ \\
\hline & Vinh 2005 & 2-day: gastrointestinal bleed (2) & 3-day: 0 \\
\hline
\end{tabular}

${ }^{a}$ Only trials reporting on complications are included.

${ }^{b}$ Zero (0) events only when specifically stated by trial author.

Table 3. Definitions of outcomes ${ }^{a}$

\begin{tabular}{|c|c|c|c|c|c|c|}
\hline Comparison & Trial & Clinical failure & $\begin{array}{l}\text { Microbiologi- } \\
\text { cal failure }\end{array}$ & Relapse & $\begin{array}{l}\text { Fever clearance } \\
\text { time }\end{array}$ & $\begin{array}{l}\text { Stool culture } \\
\text { taken }\end{array}$ \\
\hline \multirow[t]{3}{*}{$\begin{array}{l}\text { Flu- } \\
\text { oroquinolones vs } \\
\text { chlorampheni- } \\
\text { col }\end{array}$} & Abejar 1993 & Not defined & $\begin{array}{l}\text { Not } \\
\text { defined (positive } \\
\text { day } 23 \text { blood cul- } \\
\text { ture?) }\end{array}$ & Not defined & $\begin{array}{l}\text { Outcome not re- } \\
\text { ported }\end{array}$ & $\begin{array}{l}\text { Outcome not re- } \\
\text { ported }\end{array}$ \\
\hline & Arnold 1993 & $\begin{array}{l}\text { Cure } \\
\text { or improvement } \\
\text { in } 7 \text { days }\end{array}$ & $\begin{array}{l}\text { Non-eradi- } \\
\text { cation of original } \\
\text { pathogen } \\
\text { from blood sam- } \\
\text { ples be- } \\
\text { tween days } 2 \text { and } \\
9 \text { of treatment }\end{array}$ & $\begin{array}{l}\text { Reappearance of } \\
\text { signs and symp- } \\
\text { toms within } 3 \\
\text { weeks after the } \\
\text { end of treatment } \\
\text { accompanied by } \\
\text { reappear- } \\
\text { ance of pathogen } \\
\text { in blood }\end{array}$ & $\begin{array}{l}\text { Outcome not re- } \\
\text { ported }\end{array}$ & $\begin{array}{l}32 \text { to } 60 \text { days; re- } \\
\text { sults unclear }\end{array}$ \\
\hline & Bran 1991 & Not defined & Not defined & $\begin{array}{l}\text { Outcome not re- } \\
\text { ported }\end{array}$ & Not defined & $\begin{array}{l}2 \text { months after } \\
\text { treatment }\end{array}$ \\
\hline
\end{tabular}


Table 3. Definitions of outcomes ${ }^{a}$ (Continued)

\begin{tabular}{|c|c|c|c|c|c|}
\hline Cristiano 1995 & Not defined & $\begin{array}{l}\text { Blood culture } \\
\text { positive at end of } \\
\text { treatment (at } 15 \\
\text { days) }\end{array}$ & $\begin{array}{l}\text { Within } 30 \text { days } \\
\text { after end of treat- } \\
\text { ment (the } 2 \text { re- } \\
\text { lapses were blood } \\
\text { culture negative } \\
\text { and were stool } \\
\text { culture positive } \\
\text { before relapse) }\end{array}$ & Not defined & 30 days \\
\hline Gasem 2003 & $\begin{array}{l}\text { Not afebrile } \\
\text { within } 7 \text { days of } \\
\text { treatment }\end{array}$ & $\begin{array}{l}\text { Blood culture } \\
\text { positive at days } 3 \\
\text { and } 5\end{array}$ & $\begin{array}{l}\text { Reappearance of } \\
\text { fever after de- } \\
\text { fervescence dur- } \\
\text { ing hospitaliza- } \\
\text { tion (under } 14 \\
\text { days) }\end{array}$ & $\begin{array}{l}\text { Defined as first } \\
\text { day that temper- } \\
\text { ature fell }<37.5 \\
{ }^{\circ} \mathrm{C} \text { and remained } \\
\text { under for } \geq 48 \\
\text { hours }\end{array}$ & $\begin{array}{l}\text { Outcome not re- } \\
\text { ported }\end{array}$ \\
\hline Gottuzzo 1992 & $\begin{array}{l}\text { "One participant } \\
\text { who developed a } \\
\text { gastrointesti- } \\
\text { nal bleed in first } \\
36 \text { hours of treat- } \\
\text { ment was con- } \\
\text { sidered a failure" }\end{array}$ & $\begin{array}{l}\text { Outcome not re- } \\
\text { ported }\end{array}$ & Not defined & $\begin{array}{l}\text { Outcome not re- } \\
\text { ported }\end{array}$ & $\begin{array}{l}\text { Outcome not re- } \\
\text { ported }\end{array}$ \\
\hline Morelli 1992 & $\begin{array}{l}\text { Persistence of } \\
\text { fever }\end{array}$ & $\begin{array}{l}\text { Outcome not re- } \\
\text { ported }\end{array}$ & Not defined & Not defined & 3 weeks \\
\hline $\begin{array}{l}\text { Phongmany } \\
2005\end{array}$ & $\begin{array}{l}\text { Continuation of } \\
\text { symp- } \\
\text { toms and tym- } \\
\text { panic tempera- } \\
\text { ture }>38^{\circ} \mathrm{C} \text { for } \\
>10 \text { days af- } \\
\text { ter start of treat- } \\
\text { ment or contin- } \\
\text { uation of symp- } \\
\text { toms and high } \\
\text { tympanic } \\
\text { temperature }>39 \\
{ }^{\circ} \mathrm{C} \text { at } 7 \text { days af- } \\
\text { ter start of treat- } \\
\text { ment or develop- } \\
\text { ment of signs of } \\
\text { severe disease }\end{array}$ & $\begin{array}{l}\text { Outcome not re- } \\
\text { ported }\end{array}$ & $\begin{array}{l}\text { Outcome not re- } \\
\text { ported }\end{array}$ & $\begin{array}{l}\text { Time from onset } \\
\text { of treatment to } \\
\text { first recording of } \\
\text { a tympanic tem- } \\
\text { perature }<38{ }^{\circ} \mathrm{C} \\
\left(-37.5{ }^{\circ} \mathrm{C} \text { ax- }\right. \\
\text { illary) which re- } \\
\text { mained }<38{ }^{\circ} \mathrm{C} \\
\text { for } \\
48 \text { hours ('Fever } \\
\text { Clearance Time } \\
38^{\prime} \text { ) }\end{array}$ & $\begin{array}{l}\text { Outcome not re- } \\
\text { ported }\end{array}$ \\
\hline Quintero 1988 & "persistent fever" & $\begin{array}{l}\text { Outcome not re- } \\
\text { ported }\end{array}$ & $\begin{array}{l}\text { Outcome not re- } \\
\text { ported }\end{array}$ & Not defined & $\begin{array}{l}\text { Outcome not re- } \\
\text { ported }\end{array}$ \\
\hline
\end{tabular}


Table 3. Definitions of outcomes ${ }^{a}$ (Continued)

\begin{tabular}{|c|c|c|c|c|c|c|}
\hline & Yousaf 1992 & $\begin{array}{l}\text { Persistence or } \\
\text { reappear- } \\
\text { ance of all pre- } \\
\text { senting signs and } \\
\text { symptoms or in- } \\
\text { crease in severity } \\
\text { of at least } 1 \text { sign } \\
\text { or symptom or } \\
\text { both }\end{array}$ & $\begin{array}{l}\text { Persis- } \\
\text { tence of baseline } \\
\text { pathogen at day } \\
14\end{array}$ & $\begin{array}{l}\text { Outcome not re- } \\
\text { ported }\end{array}$ & $\begin{array}{l}\text { Outcome not re- } \\
\text { ported }\end{array}$ & $\begin{array}{l}\text { Outcome not re- } \\
\text { ported }\end{array}$ \\
\hline $\begin{array}{l}\text { Flu- } \\
\text { oroquinolone vs } \\
\text { ampicillin }\end{array}$ & Flores 1994 & $\begin{array}{l}\text { At end of treat- } \\
\text { ment }\end{array}$ & $\begin{array}{l}\text { At end of treat- } \\
\text { ment }\end{array}$ & $\begin{array}{l}\text { Outcome not re- } \\
\text { ported }\end{array}$ & $\begin{array}{l}\text { Outcome not re- } \\
\text { ported }\end{array}$ & $\begin{array}{l}\text { Outcome not re- } \\
\text { ported }\end{array}$ \\
\hline \multirow[t]{2}{*}{$\begin{array}{l}\text { Fluoro- } \\
\text { quinolone vs co- } \\
\text { trimoxazole }\end{array}$} & Hajji 1988 & $\begin{array}{l}\text { Fever and pres- } \\
\text { ence } \\
\text { of clinical symp- } \\
\text { toms and posi- } \\
\text { tive cultures }\end{array}$ & $\begin{array}{l}\text { Positive cultures } \\
\text { at days } 4,15 \text {, and } \\
30\end{array}$ & $\begin{array}{l}\text { Reappearance of } \\
\text { fever, clinical } \\
\text { symptoms, } \\
\text { and/or bacter- } \\
\text { aemia at days } 4 \text {, } \\
15 \text {, and } 30\end{array}$ & $\begin{array}{l}\text { Time for rec- } \\
\text { tal temperature } \\
\text { to be sustained } \leq \\
37.5{ }^{\circ} \mathrm{C} \text { for } \geq 2 \\
\text { days }\end{array}$ & 30 days \\
\hline & Limson 1989 & $\begin{array}{l}\text { Persis- } \\
\text { tent fever or no } \\
\text { improvement in } \\
\text { symptoms after } 5 \\
\text { days of therapy }\end{array}$ & $\begin{array}{l}\text { Positive cultures } \\
\text { during and after } \\
\text { therapy }\end{array}$ & $\begin{array}{l}\text { Outcome not re- } \\
\text { ported }\end{array}$ & $\begin{array}{l}\text { Outcome not re- } \\
\text { ported }\end{array}$ & $\begin{array}{l}\text { Outcome not re- } \\
\text { ported }\end{array}$ \\
\hline \multirow[t]{2}{*}{$\begin{array}{l}\text { Flu- } \\
\text { oroquinolone vs } \\
\text { azithromycin }\end{array}$} & Dolecek 2008 & $\begin{array}{l}\text { Persistence of } \\
\text { fever and symp- } \\
\text { toms } 2 \text { days after } \\
\text { the end of treat- } \\
\text { ment, ie on day } \\
10\end{array}$ & $\begin{array}{l}\text { Positive } \\
\text { blood culture on } \\
\text { day } 7 \text { to } 9 \text { after } \\
\text { the start of treat- } \\
\text { ment }\end{array}$ & $\begin{array}{l}\text { Symptoms and } \\
\text { signs suggestive } \\
\text { of } \\
\text { typhoid fever } \\
\text { within } 1 \text { month } \\
\text { after completion } \\
\text { of treat- } \\
\text { ment (only cul- } \\
\text { ture positive data } \\
\text { extracted) }\end{array}$ & $\begin{array}{l}\text { Time from start } \\
\text { of antibi- } \\
\text { otic treatment to } \\
\text { when the axil- } \\
\text { lary temperature } \\
\text { first fell } \leq 37.5 \\
{ }^{\circ} \mathrm{C} \text { and remained } \\
\text { there for at least } \\
48 \text { hours }\end{array}$ & $\begin{array}{l}\text { Follow ups at } 1 \text {, } \\
3 \text {, and } 6 \text { months; } \\
\text { participants who } \\
\text { attended at least } \\
2 \text { consecutive } \\
\text { follow-up visits } \\
\text { were evaluated }\end{array}$ \\
\hline & Chinh 2000 & $\begin{array}{l}\text { Persistence of } \\
\text { fever and symp- } \\
\text { toms for > } 5 \\
\text { days after the end } \\
\text { of treatment or } \\
\text { development of } \\
\text { severe complica- } \\
\text { tions (severe gas- } \\
\text { trointestinal } \\
\text { bleed, intestinal } \\
\text { perforation, visi- }\end{array}$ & $\begin{array}{l}\text { Isolation of } S \text {. } \\
\text { Typhi/S. Paraty- } \\
\text { phi from blood } \\
\text { or other sterile } \\
\text { site after comple- } \\
\text { tion of treatment }\end{array}$ & $\begin{array}{l}\text { Recurrence of } \\
\text { signs and symp- } \\
\text { toms suggestive } \\
\text { of enteric fever } \\
\text { after discharge at } \\
4 \text { to } 6 \text { weeks of } \\
\text { follow up }\end{array}$ & $\begin{array}{l}\text { Time from start } \\
\text { of treatment un- } \\
\text { til body temper- } \\
\text { ature fell }<37.5 \\
{ }^{\circ} \mathrm{C} \text { and remained } \\
\text { at } \leq 37.5^{\circ} \mathrm{C} \text { for } \\
48 \text { hours }\end{array}$ & $\begin{array}{l}\text { Days } 2 \text { to } 3 \text { after } \\
\text { end of treatment }\end{array}$ \\
\hline
\end{tabular}


Table 3. Definitions of outcomes ${ }^{a}$ (Continued)

\begin{tabular}{|c|c|c|c|c|c|c|}
\hline & & $\begin{array}{l}\text { ble jaundice, my- } \\
\text { ocardi- } \\
\text { tis, renal failure, } \\
\text { shock, coma) } \\
\text { during treatment } \\
\text { requiring change } \\
\text { in treatment }\end{array}$ & & & & \\
\hline & Girgis 1999 & $\begin{array}{l}\text { Lack of resolu- } \\
\text { tion of symp- } \\
\text { toms by day } 7 \text { or } \\
\text { development of } \\
\text { major complica- } \\
\text { tions of typhoid } \\
\text { fever after } 5 \text { days } \\
\text { of therapy }\end{array}$ & $\begin{array}{l}\text { Blood } \\
\text { culture positive } \\
\text { for } S \text {. Typhi } / S \text {. } \\
\text { Paratyphi on day } \\
10\end{array}$ & $\begin{array}{l}\text { Re- } \\
\text { currence of fever } \\
\text { with signs/symp- } \\
\text { toms of typhoid } \\
\text { fever in } 4 \text { weeks } \\
\text { of therapy com- } \\
\text { pletion and cul- } \\
\text { ture positive }\end{array}$ & $\begin{array}{l}\text { First day } \\
\text { on which max- } \\
\text { imum tempera- } \\
\text { ture } \leq 38^{\circ} \mathrm{C} \text { and } \\
\text { at this level for } \geq \\
48 \text { hours }\end{array}$ & 1 month \\
\hline & Parry 2007 & $\begin{array}{l}\text { Presence of fever } \\
\text { and at least } \\
1 \text { other typhoid } \\
\text { related symptom } \\
\text { for }>7 \text { days af- } \\
\text { ter start of treat- } \\
\text { ment or devel- } \\
\text { opment of severe } \\
\text { complications } \\
\text { (severe gastroin- } \\
\text { testinal bleeding, } \\
\text { perforation, visi- } \\
\text { ble jaundice, my- } \\
\text { ocarditis, pneu- } \\
\text { monia, renal fail- } \\
\text { ure, shock, or al- } \\
\text { tered conscious- } \\
\text { ness level, during } \\
\text { treatment } \\
\text { requiring change } \\
\text { in therapy }\end{array}$ & $\begin{array}{l}\text { Isolation } \\
\text { of } S \text {. Typhi or } S \text {. } \\
\text { Paratyphi from } \\
\text { blood or sterile } \\
\text { site after comple- } \\
\text { tion of treatment }\end{array}$ & $\begin{array}{l}\text { Recurrence } \\
\text { of symptoms or } \\
\text { signs sug- } \\
\text { gestive of enteric } \\
\text { fever within 4- } \\
\text { week period af- } \\
\text { ter patient had } \\
\text { been discharged } \\
\text { well from hospi- } \\
\text { tal accompanied } \\
\text { by positive blood } \\
\text { culture for } S \text {. Ty- } \\
\text { phi or } S \text {. Paraty- } \\
\text { phi }\end{array}$ & $\begin{array}{l}\text { Time from start } \\
\text { of treatment un- } \\
\text { til body temper- } \\
\text { ature reached } \leq \\
37.5^{\circ} \mathrm{C} \text { and re- } \\
\text { mained at this } \\
\text { for } 48 \text { hours }\end{array}$ & $\begin{array}{l}\text { After end of ini- } \\
\text { tial } 7 \text {-day treat- } \\
\text { ment and be- } \\
\text { fore hospital dis- } \\
\text { charge (with iso- } \\
\text { late having } \\
\text { the same suscep- } \\
\text { tibility pattern as } \\
\text { original isolate) }\end{array}$ \\
\hline $\begin{array}{l}\text { Fluoro- } \\
\text { quinolone vs ce- } \\
\text { fixime }\end{array}$ & Cao 1999 & $\begin{array}{l}\text { Deterio- } \\
\text { ration in clinical } \\
\text { condition or fail- } \\
\text { ure of resolution } \\
\text { of symptoms } \\
\text { requiring further } \\
\text { treatment }\end{array}$ & $\begin{array}{l}\text { Blood culture } \\
\text { positive for } S \text {. } \\
\text { Typhi after com- } \\
\text { pletion of treat- } \\
\text { ment }\end{array}$ & $\begin{array}{l}\text { Symptoms } \\
\text { suggestive of ty- } \\
\text { phoid fever with } \\
\text { a positive blood } \\
\text { or bone marrow } \\
\text { culture up to } 4 \\
\text { weeks after dis- }_{\text {charge }^{b}}\end{array}$ & $\begin{array}{l}\text { Time from onset } \\
\text { of treatment un- } \\
\text { til fever was } 37.5 \\
{ }^{\circ} \mathrm{C} \text { or below for } \\
\text { at least } 24 \text { hours }\end{array}$ & $\begin{array}{l}1 \text { month mostly, } \\
\text { few seen after a } \\
\text { longer period }\end{array}$ \\
\hline
\end{tabular}

Fluoroquinolones for treating typhoid and paratyphoid fever (enteric fever) (Review)

Copyright @ 2008 The Cochrane Collaboration. Published by John Wiley \& Sons, Ltd. 
Table 3. Definitions of outcomes ${ }^{a}$ (Continued)

\begin{tabular}{|c|c|c|c|c|c|c|}
\hline & Pandit 2007 & $\begin{array}{l}\text { Any severe com- } \\
\text { plication, persis- } \\
\text { tence of fever (> } \\
38{ }^{\circ} \mathrm{C} \text { ), persis- } \\
\text { tence of symp- } \\
\text { toms for }>7 \text { days } \\
\text { af- } \\
\text { ter start of treat- } \\
\text { ment, requiring } \\
\text { additional or res- } \\
\text { cue treatment }\end{array}$ & $\begin{array}{l}\text { Blood cul- } \\
\text { ture positive on } \\
\text { day } 10\end{array}$ & $\begin{array}{l}\text { Fever with blood } \\
\text { culture positive } \\
\text { within a month } \\
\text { of completing } \\
\text { treatment (pa- } \\
\text { tients given res- } \\
\text { cue treatment or } \\
\text { prolonged treat- } \\
\text { ment were ex- } \\
\text { cluded) }\end{array}$ & $\begin{array}{l}\text { Time to } 1 \text { st drop } \\
\text { in oral tempera- } \\
\text { ture } \leq \\
37.5^{\circ} \mathrm{C} \text { remain- } \\
\text { ing } \leq 37.5^{\circ} \mathrm{C} \text { for } \\
48 \text { hours }\end{array}$ & 1 month \\
\hline & Yu 1998 & $\begin{array}{l}\text { "ineffi- } \\
\text { cient" (categories } \\
\text { included in as- } \\
\text { sessment of clin- } \\
\text { ical effectiveness } \\
\text { were "cure, effec- } \\
\text { tive, improved } \\
\text { and inefficient") }\end{array}$ & $\begin{array}{l}\text { Eradicated (bac- } \\
\text { teria elimination } \\
\text { rate) }\end{array}$ & $\begin{array}{l}\text { Carrying bacte- } \\
\text { ria at } 3 \text { months } \\
\text { follow up }\end{array}$ & Not defined & Not defined \\
\hline \multirow[t]{3}{*}{$\begin{array}{l}\text { Fluoro- } \\
\text { quinolone vs cef- } \\
\text { triaxone }\end{array}$} & Smith 1994 & $\begin{array}{l}\text { Acute treatment } \\
\text { failure as contin- } \\
\text { uing symptoms } \\
\text { and fever for at } \\
\text { least } 7 \text { days after } \\
\text { starting the treat- } \\
\text { ment regimen }\end{array}$ & $\begin{array}{l}\text { Blood culture } \\
\text { positive at day } 8\end{array}$ & $\begin{array}{l}\text { Re- } \\
\text { currence of fever } \\
\text { and symptoms in } \\
\text { the period up to } \\
6 \text { weeks after dis- } \\
\text { charge with } \\
\text { a positive blood } \\
\text { or bone marrow } \\
\text { culture }^{b}\end{array}$ & $\begin{array}{l}\text { Time } \\
\text { to defervescence } \\
\text { to }<37.5^{\circ} \mathrm{C} \text { for } \\
\text { at least } 48 \text { hours }\end{array}$ & 4 to 6 weeks \\
\hline & Tran 1994 & $\begin{array}{l}\text { No reduction of } \\
\text { maximum daily } \\
\text { temperature } \\
\text { to }<37.5^{\circ} \mathrm{C} \text { nor } \\
\text { complete disap- } \\
\text { pearance of all } \\
\text { other signs and } \\
\text { symptoms } \\
\text { within } 14 \text { days } \\
\text { and with clini- } \\
\text { cal evidence of } \\
\text { infection during } \\
\text { further follow up }\end{array}$ & $\begin{array}{l}\text { Blood, bone } \\
\text { marrow, or stool } \\
\text { culture within } \\
14 \text { days, and all } \\
\text { culture negative } \\
\text { for at least } 21 \\
\text { days }\end{array}$ & $\begin{array}{l}\text { Re- } \\
\text { turn of fever and } \\
\text { symptoms up to } \\
4 \text { weeks after dis- } \\
\text { charge with a } \\
\text { blood or bone } \\
\text { marrow culture } \\
\text { positive }^{b}\end{array}$ & $\begin{array}{l}\text { Time until fever } \\
\text { reached }<37.5 \\
{ }^{\circ} \mathrm{C}\end{array}$ & 1 month \\
\hline & Wallace 1993 & $\begin{array}{l}\text { Fever }>38^{\circ} \mathrm{C} \text { af- } \\
\text { ter } 7 \text { days of ther- } \\
\text { apy or who de- } \\
\text { teriorated clini- }\end{array}$ & $\begin{array}{l}\text { Blood culture } \\
\text { positive at day } 3\end{array}$ & $\begin{array}{l}\text { Readmission for } \\
\text { typhoid within } 2 \\
\text { months of dis- } \\
\text { charge with stool }\end{array}$ & Not defined & $\begin{array}{l}\text { Days } 1,7 \text {, and } \\
28 \text {; results un- } \\
\text { clear }\end{array}$ \\
\hline
\end{tabular}


Table 3. Definitions of outcomes ${ }^{a}$ (Continued)

\begin{tabular}{|c|c|c|c|c|c|c|}
\hline & & $\begin{array}{l}\text { cally after } 5 \text { full } \\
\text { days }\end{array}$ & & $\begin{array}{l}\text { or blood culture } \\
\text { pos- } \\
\text { itive for } S \text {. Typhi } \\
\text { of the same an- } \\
\text { tibiogram ( } 1 \text { re- } \\
\text { lapse had both } \\
\text { stool and blood } \\
\text { culture positive) }\end{array}$ & & \\
\hline \multirow[t]{2}{*}{$\begin{array}{l}\text { Nor- } \\
\text { floxacin vs chlo- } \\
\text { ramphenicol }\end{array}$} & Nalin 1987 & Not defined & Not defined & $\begin{array}{l}\text { Outcome not re- } \\
\text { ported }\end{array}$ & $\begin{array}{l}\text { First day } \\
\text { at which temper- } \\
\text { ature }<37.5^{\circ} \mathrm{C}\end{array}$ & Not defined \\
\hline & Sarma 1991 & $\begin{array}{l}\text { Persistence of all } \\
\text { signs and symp- } \\
\text { toms of infection }\end{array}$ & $\begin{array}{l}\text { Positive blood } \\
\text { culture at } 7 \text { days }\end{array}$ & $\begin{array}{l}\text { Recurrence of ill- } \\
\text { ness within } 14 \\
\text { days of comple- } \\
\text { tion of treatment }\end{array}$ & $\begin{array}{l}\text { Disap- } \\
\text { pearance of fever } \\
\text { after therapy and } \\
\text { maintenance of } \\
\text { normal tempera- } \\
\text { ture with clinical } \\
\text { cure and blood } \\
\text { sterilization }\end{array}$ & 56 days \\
\hline $\begin{array}{l}\text { Norfloxacin vs } \\
\text { ceftriaxone }\end{array}$ & Huai 2000 & $\begin{array}{l}\text { Fever after } 7 \text { days } \\
\text { of } \\
\text { treatment, posi- } \\
\text { tive bacteria, ex- } \\
\text { istence of symp- } \\
\text { toms and com- } \\
\text { plications }\end{array}$ & $\begin{array}{l}\text { Outcome not re- } \\
\text { ported }\end{array}$ & $\begin{array}{l}\text { Relapse after } 1 \\
\text { month of follow } \\
\text { up }\end{array}$ & Not defined & $\begin{array}{l}\text { Outcome not re- } \\
\text { ported }\end{array}$ \\
\hline \multirow[t]{3}{*}{$\begin{array}{l}\text { Norfloxacin } \\
\text { vs another fluo- } \\
\text { roquinolone }\end{array}$} & Bai 1995 & $\begin{array}{l}\text { No } \\
\text { effect or deterio- } \\
\text { rated after } 3 \text { days } \\
\text { of treatment }\end{array}$ & $\begin{array}{l}\text { Outcome not re- } \\
\text { ported }\end{array}$ & $\begin{array}{l}\text { Outcome not re- } \\
\text { ported }\end{array}$ & $\begin{array}{l}\text { Restoration of } \\
\text { normal tempera- } \\
\text { ture }\end{array}$ & $\begin{array}{l}\text { Outcome not re- } \\
\text { ported }\end{array}$ \\
\hline & Jia 1994 & $\begin{array}{l}\text { No effect or de- } \\
\text { teriorated }\end{array}$ & $\begin{array}{l}\text { Outcome not re- } \\
\text { ported }\end{array}$ & $\begin{array}{l}\text { Outcome not re- } \\
\text { ported }\end{array}$ & $\begin{array}{l}\text { Restoration of } \\
\text { normal tempera- } \\
\text { ture }\end{array}$ & $\begin{array}{l}\text { Outcome not re- } \\
\text { ported }\end{array}$ \\
\hline & Xiao 1991 & $\begin{array}{l}\text { Temperature not } \\
\text { de- } \\
\text { creased or even } \\
\text { increased, clini- } \\
\text { cal symp- } \\
\text { toms were im- } \\
\text { proved or dete- } \\
\text { riorated after } 1 \\
\text { week of treat- } \\
\text { ment }\end{array}$ & $\begin{array}{l}\text { Outcome not re- } \\
\text { ported }\end{array}$ & $\begin{array}{l}\text { Outcome not re- } \\
\text { ported }\end{array}$ & Not defined & $\begin{array}{l}\text { Outcome not re- } \\
\text { ported }\end{array}$ \\
\hline
\end{tabular}


Table 3. Definitions of outcomes ${ }^{a}$ (Continued)

\begin{tabular}{|c|c|c|c|c|c|c|}
\hline & Yang 1991 & $\begin{array}{l}\text { Temperature not } \\
\text { decreased after } 8 \\
\text { or } \\
\text { more days treat- } \\
\text { ment or changed } \\
\text { to other treat- } \\
\text { ment }\end{array}$ & $\begin{array}{l}\text { Tem- } \\
\text { perature back to } \\
\text { normal but bac- } \\
\text { teria culture pos- } \\
\text { itive from faeces }\end{array}$ & $\begin{array}{l}\text { Tempera- } \\
\text { ture back to nor- } \\
\text { mal after treat- } \\
\text { ment but after } 2 \\
\text { to } 4 \text { weeks fever } \\
\text { started again and } \\
\text { blood } \\
\text { cultures positive } \\
\text { and } S \text {. Typhi iso- } \\
\text { lated }\end{array}$ & Not defined & $\begin{array}{l}\text { At } \\
\text { "convalescence" }\end{array}$ \\
\hline \multirow[t]{5}{*}{$\begin{array}{l}\text { Different dura- } \\
\text { tions of fluoro- } \\
\text { quinolones }\end{array}$} & Alam 1995 & $\begin{array}{l}\text { Lack of improve- } \\
\text { ment or deterio- } \\
\text { ration in clinical } \\
\text { condition during } \\
\text { treatment }\end{array}$ & $\begin{array}{l}\text { Growth of } S \text {. Ty- } \\
\text { phi or } S \text {. Paraty- } \\
\text { phi in blood in } \\
\text { first follow up } \\
\text { (day 3) }\end{array}$ & $\begin{array}{l}\text { Recurrence } \\
\text { of febrile illness } \\
\text { with growth of } S \text {. } \\
\text { Typhi } \\
\text { or } S \text {. Paratyphi in } \\
\text { blood culture af- } \\
\text { ter initial cure }\end{array}$ & $\begin{array}{l}\text { Time to return of } \\
\text { oral temperature } \\
\text { to } \leq 37.5^{\circ} \mathrm{C} \text { af- } \\
\text { ter initiation of } \\
\text { therapy and re- } \\
\text { mained so for at } \\
\text { least } 48 \text { hours }\end{array}$ & $\begin{array}{l}\text { Second follow } \\
\text { up (at } 2 \text { months) }\end{array}$ \\
\hline & Duong 1995 & $\begin{array}{l}\text { No } \\
\text { improvement or } \\
\text { reappearance of } \\
\text { signs and symp- } \\
\text { toms at least } 48 \\
\text { hours after end } \\
\text { of treatment }\end{array}$ & $\begin{array}{l}\text { Blood or bone } \\
\text { marrow culture } \\
\text { positive } 48 \text { hours } \\
\text { after last dose of } \\
\text { fleroxacin }\end{array}$ & $\begin{array}{l}\text { Blood culture } \\
\text { positive within } 1 \\
\text { month follow up }\end{array}$ & Not defined & $\begin{array}{l}\text { Outcome not re- } \\
\text { ported }\end{array}$ \\
\hline & Kalo 1997 & Fever at day 5 & $\begin{array}{l}\text { Blood culture } \\
\text { positive at day } 4\end{array}$ & $\begin{array}{l}\text { Re- } \\
\text { lapse during hos- } \\
\text { pitalization and } \\
2 \text { month follow } \\
\text { up }\end{array}$ & $\begin{array}{l}\text { Outcome not re- } \\
\text { ported }\end{array}$ & Days 7 to 12 \\
\hline & Nguyen 1997 & $\begin{array}{l}\text { Continuing } \\
\text { fever and symp- } \\
\text { toms for } 7 \text { days } \\
\text { after } \\
\text { the start of treat- } \\
\text { ment or deterio- } \\
\text { ration in clinical } \\
\text { condition before } \\
7 \text { days that war- } \\
\text { ranted further } \\
\text { treatment }\end{array}$ & $\begin{array}{l}\text { Blood or bone } \\
\text { marrow culture } \\
\text { positive after end } \\
\text { of treatment be- } \\
\text { fore discharge }\end{array}$ & $\begin{array}{l}\text { Recurrent } \\
\text { fever and symp- } \\
\text { toms with bone } \\
\text { marrow or blood } \\
\text { culture positive } \\
\text { mostly up to } 6 \\
\text { weeks after dis- } \\
\text { charge }^{b}\end{array}$ & $\begin{array}{l}\text { Time at which } \\
\text { fever fell below } \\
37.5{ }^{\circ} \mathrm{C} \text { for at } \\
\text { least } 24 \text { hours }\end{array}$ & $\begin{array}{l}\text { Usually } 6 \text { weeks } \\
\text { (occasionally up } \\
\text { to } 12 \text { weeks) }\end{array}$ \\
\hline & Tran 1995 & $\begin{array}{l}\text { Per- } \\
\text { sistent fever and } \\
\text { symptoms for }> \\
7 \text { days after start }\end{array}$ & $\begin{array}{l}\text { Blood or bone } \\
\text { marrow culture } \\
\text { positive after end } \\
\text { of treatment }\end{array}$ & $\begin{array}{l}\text { Symptoms since } \\
\text { study with pos- } \\
\text { itive blood cul- } \\
\text { ture }\end{array}$ & Not defined & 1 month \\
\hline
\end{tabular}


Table 3. Definitions of outcomes ${ }^{a}$ (Continued)

of treatment

\begin{tabular}{|c|c|c|c|c|c|}
\hline Unal 1996 & $\begin{array}{l}\text { Continued or } \\
\text { worsening symp- } \\
\text { toms after } 7 \text { days } \\
\text { of therapy }\end{array}$ & $\begin{array}{l}\text { Failure to eradi- } \\
\text { cate organism }\end{array}$ & $\begin{array}{l}\text { Similar signs and } \\
\text { symp- } \\
\text { toms after appar- } \\
\text { ently being cured } \\
\text { for a month (the } \\
\text { participant had } \\
\text { a positive stool } \\
\text { culture) }\end{array}$ & $\begin{array}{l}\text { Time for tem- } \\
\text { perature to be } \\
\text { below } 37.5{ }^{\circ} \mathrm{C} \\
\text { for at least } 48 \\
\text { hours }\end{array}$ & $\begin{array}{l}1 \text { month; results } \\
\text { unclear }\end{array}$ \\
\hline Vinh 1996 & $\begin{array}{l}\text { Continued } \\
\text { fever and symp- } \\
\text { toms for }>7 \text { days } \\
\text { after treatment }\end{array}$ & $\begin{array}{l}\text { Positive blood } \\
\text { culture or bone } \\
\text { marrow cul- } \\
\text { ture for } S \text {. Typhi } \\
\text { taken }>48 \text { hours } \\
\text { after the last dose } \\
\text { of treatment }\end{array}$ & $\begin{array}{l}\text { Recurrence of } \\
\text { fever and symp- } \\
\text { toms with posi- } \\
\text { tive } \\
\text { blood or bone } \\
\text { marrow culture } \\
\text { up to } 6 \text { weeks ( } 26 \\
\text { participants fol- } \\
\text { lowed up to } 12 \\
\text { weeks) after dis- } \\
\text { chargeb }\end{array}$ & $\begin{array}{l}\text { Time from start } \\
\text { of treatment un- } \\
\text { til axillary tem- } \\
\text { perature fell be- } \\
\text { low } 37.5^{\circ} \mathrm{C} \text { and } \\
\text { remained below } \\
\text { this level for }>48 \\
\text { hours }\end{array}$ & $\begin{array}{l}4 \text { to } 6 \text { weeks (for } \\
66 \text { participants); } \\
\text { and at } 3 \text { months } \\
\text { (for } 26 \text { partici- } \\
\text { pants) }\end{array}$ \\
\hline Vinh 2005 & $\begin{array}{l}\text { Fever and symp- } \\
\text { toms persisting } \\
\text { for } \geq 7 \text { days af- } \\
\text { ter start of ther- } \\
\text { apy, or develop- } \\
\text { ment of severe or } \\
\text { complicated dis- } \\
\text { ease }\end{array}$ & $\begin{array}{l}\text { Blood culture } \\
\text { positive for same } \\
\text { organism } \\
\text { between } 7 \text { to } 28 \\
\text { days after com- } \\
\text { pletion of ther- } \\
\text { apy }\end{array}$ & $\begin{array}{l}\text { Recur- } \\
\text { rence of typhoid } \\
\text { fever symptoms } \\
\text { usually with pos- } \\
\text { itive blood cul- } \\
\text { ture after hospi- } \\
\text { tal discharge un- } \\
\text { til } 28 \text { days post } \\
\text { discharge (only } \\
\text { data for blood } \\
\text { culture-con- } \\
\text { firmed relapse } \\
\text { extracted) }\end{array}$ & $\begin{array}{l}\text { Period from start } \\
\text { of treatment un- } \\
\text { til tempera- } \\
\text { ture remained at } \\
\text { or below } 37.5^{\circ} \mathrm{C} \\
\text { for at least } 48 \\
\text { hours }\end{array}$ & $\begin{array}{l}\text { Immediately af- } \\
\text { ter treatment }\end{array}$ \\
\hline
\end{tabular}

S. Typhi/S. Paratyphi: Salmonella enterica serovar Typhi/Paratyphi.

${ }^{a}$ All definitions as stated or implied by trial authors.

${ }^{b}$ With an organism with the same sensitivity pattern, ribotype, and plasmid profile as the original isolate. 


\section{H I S T O R Y}

Protocol first published: Issue 4, 2003

Review first published: Issue 2, 2005

\section{CONTRIBUTIONSOFAUTHORS}

Durrane Thaver conducted the literature search, extracted and entered data, analysed results, and wrote the review. Asma Azmatullah co-extracted and double-entered data, and assisted in literature search, data analysis and writing the review (for this update). Ali Madni co-extracted data (for Thaver 2005). Anita Zaidi was consulted to resolve disagreements. Anita Zaidi, Julia Critchley, and Zulfiqar Bhutta provided technical input and edited the manuscript.

\section{DECLARATIONSOF INTEREST}

None known. Professor ZA Bhutta has been involved in typhoid therapy trials in children, none of which involved fluoroquinolones.

\section{SOURCES OF SUPPORT}

\section{Internal sources}

- The Aga Khan University Hospital, Pakistan.

\section{External sources}

- Department for International Development (DFID), UK.

\section{DIFFERENCES BETWEEN PROTOCOLAND REVIEW}

We changed the title from 'Fluoroquinolones for treating enteric fever' and added "any sterile anatomic site" to the definition of the "relapse" primary outcome.

\section{INDEX TERMS}

\section{Medical Subject Headings (MeSH)}

Anti-Bacterial Agents [adverse effects; * therapeutic use]; Fluoroquinolones [adverse effects; * therapeutic use]; Norfloxacin [therapeutic use]; Paratyphoid Fever [*drug therapy]; Randomized Controlled Trials as Topic; Treatment Outcome; Typhoid Fever [*drug therapy] 
MeSH check words

Adult; Child; Humans

Fluoroquinolones for treating typhoid and paratyphoid fever (enteric fever) (Review)

Copyright @ 2008 The Cochrane Collaboration. Published by John Wiley \& Sons, Ltd. 